\title{
A Systematic Study of Actinide Production from the Interactions of Heavy Ions with ${ }^{248} \mathrm{Cm}$
}

by

John David Leyba

Ph.D. Thesis

September 7, 1990

Department of Chemistry

University of California, Berkeley

and

Nuclear Science Division

Lawrence Berkeley Laboratory

1 Cyclotron Road

Berkeley, CA 94720

This work was supported in part by the Director, Office of Energy Research, Division of Nuclear Physics of the Office of High Energy and Nuclear Physics of the U.S. Department of Energy under Contract No. DE-AC03-76SF00098. 


\title{
A Systematic Study of Actinide Production from the Interactions of Heavy Ions with $248 \mathrm{Cm}$
}

by

\author{
John David Leyba
}

\begin{abstract}
Production cross sections for heavy actinides produced from the interactions of ${ }^{12} \mathrm{C}$, ${ }^{31} \mathrm{P},{ }^{40} \mathrm{Ar}$, and ${ }^{44} \mathrm{Ca}$ ions with ${ }^{248} \mathrm{Cm}$ were measured at energies ranging from 0.98 to $1.35 \mathrm{X}$ Coulomb barrier. The recoiling reaction products were collected in copper or gold catcher foils located near the ${ }^{248} \mathrm{Cm}$ target. Separate fractions of Bk, Cf, Es, Fm, and $\mathrm{Md}$ were obtained from a radiochemical separation procedure. For the ${ }^{12} \mathrm{C}$ system, a $\mathrm{He} / \mathrm{KCl}$ jet was used to transport the recoiling No activities of interest to a rotating wheel system. The isotopic distributions of the actinide products were found to be essentially symmetric about the maximum with full-widths-at-half-maximum of approximately 2.5 mass units. Isotopic distributions of the ${ }^{12} \mathrm{C},{ }^{31} \mathrm{P},{ }^{40} \mathrm{Ar}$, and ${ }^{44} \mathrm{Ca}$ systems were found to be very similar to the $40,48 \mathrm{Ca}$ systems studied previously. The maxima of the isotopic distributions generally occurred for those reaction channels which involved the exchange of the fewest number of nucleons between the target and projectile for which the calculated excitation energy was a positive quantity. Additionally, the maxima of the excitation functions occurred at those projectile energies which were consistent with the calculated reaction barriers based upon a binary reaction mechanism. The experimental data from the four systems investigated were compared to several models of heavy ion interactions including a damped reaction mechanism, compound nucleus formation and subsequent particle evaporation, and classical partial wave calculations for binary systems.
\end{abstract}




\section{DEDICATION}

I would like to dedicate this thesis to the world's best teacher, Dr. John Hoffman. It is because of this man's enthusiasm and excellent teaching ability that I decided to go into chemistry. I thank him for giving me an excellent foundation of fundamental skills, without which, I could not have achieved my dreams. I hope that he will continue to be a role model for many other students in years to come. Keep up the great work! 


\section{ACKNOWLEDGEMENTS}

First and foremost I would like to thank my parents, Guillermo Leyba and Ann Leyba for encouraging me to achieve my own goals and ambitions and for making me do my homework before I could watch television. I would also like to thank my mom for providing me with a place to live when I no longer had a home. I appreciated all of the support that I received from my family including Tony, Mark, Liz, Kenny (thanks for the \$), Sally, Kathy, and Scoo.

I would like to thank Dr. Darleane Hoffman for giving me the opportunity to study under her. I will always be thankful for all that she has given me (except for the sore arm).

I would also like to thank Dr. Kenneth Gregorich for providing me with all of the answers when I needed them (which was often).

I would like to thank all of the present and past members of SHEIKS including Diana Lee, Matti Nurmia, Al Ghiorso, Roger Henderson, Bob Chasteler, Dianne Bennett, Howard Hall, Carolyn Gannett, Bob Chadwick, Ken (Flipper) Czerwinski, Bobby Kadkhodayan, Steve Kreek, Nancy Hannink, Chris Kacher, Otto Lee, Lithium Lin, George Haynes, and Beth Brady.

Finally, I would like to thank the staff and crew of the Lawrence Berkeley Laboratory 88-Inch Cyclotron for the excellent heavy ion beams that they provided me.

This work was supported in part by the Director, Office of Energy Research, Division of Nuclear Physics of the Office of High Energy and Nuclear Physics of the U.S. Department of Energy under Contract DE-AC03-76SF00098. 


\section{TABLE OF CONTENTS}

I. Introduction 1

A. Heavy Ion Reactions 1

1. General Features 1

2. Classical Mechanics: An Introduction 1

3. Elastic and Inelastic Scattering 5

4. Transfer Reactions 5

5. Deeply Inelastic Reactions 6

6. Complete Fusion Reactions 6

B. A Brief History of Transfer Reactions 6

C. Transfer Reaction Studies Using ${ }^{248} \mathrm{Cm}$ as a Target 9

D. Rationale Behind Projectile Selection $\quad 10$

Chapter I Table 13

Chapter I Figure Captions $\quad 14$

Chapter I Figures $\quad 15$

$\begin{array}{ll}\text { II. Experimental } & 17\end{array}$

$\begin{array}{ll}\text { A. Target Requirements } & 17\end{array}$

B. Target System 17

C. Catcher Foil Collection Efficiency 18

D. Experimental Conditions 22

E. Manual Chemical Separations 22

1. Anion Exchange Chromatography 23

2. Cation Exchange Chromatography 23 $\alpha$-Hydroxyisobutyrate Separations $\quad 24$

3. Reverse Phase HDEHP Column Chromatography 25

a. Separation of the Actinides from the Lanthanides 25

b. Elution of Actinide Fractions Containing Short-Lived Nuclides Via Reverse Phase Column Chromatography 25

4. Recoil Atom Collection Foils 26

a. Chemical Procedure for Copper Catcher Foils 26

b. Chemical Procedure for Gold Catcher Foils 28

F. Automated Chromatographic Chemical Element Separator System 29 
G. Data Acquisition 30

1. Merry-Go-Round Realtime Data Acquisition and Graphics System 30

2. Counting Procedure 31

H. Data Processing and Calculations 32

1. Processing of Alpha Data 32

2. Processing of Gamma Data 33

3. Calculation of Cross Sections 33

Chapter II Tables 36

Chapter II Figure Captions $\quad 45$

$\begin{array}{ll}\text { Chapter II Figures } & 47\end{array}$

$\begin{array}{ll}\text { III. Results and Discussion } & 61\end{array}$

A. Cross Sections and Excitation Functions 61

B. Excitation Energies 63

C. Division of Energy 65

D. ${ }^{12} \mathrm{C}-248 \mathrm{Cm}$ System 66

1. Isotopic Distributions 66

2. Excitation Functions 67

a. Excitation Functions for Bk Isotopes 67

b. Excitation Functions for Cf Isotopes 68

c. Excitation Functions for Es Isotopes 68

d. Excitation Functions for Fm Isotopes 68

e. $F_{t}$ and $E_{t}$ Values $\quad 69$

E. ${ }^{31 \mathrm{P}-248 \mathrm{Cm} \text { System }} 69$

1. Isotopic Distributions 69

2. Excitation Functions 70

a. Excitation Functions for Bk Isotopes 70

b. Excitation Functions for Cf Isotopes 70

c. Excitation Functions for Es Isotopes 71

d. Excitation Functions for Fm Isotopes 71

e. $F_{t}$ and $E_{t}$ Values 71

F. ${ }^{40} \mathrm{Ar}-248 \mathrm{Cm}$ System $\quad 72$

1. Isotopic Distributions 72

2. Excitation Functions 72

a. Excitation Functions for Bk Isotopes 72 
b. Excitation Functions for Cf Isotopes 72

c. Excitation Functions for Es Isotopes 73

d. Excitation Functions for Fm Isotopes 74

e. $F_{t}$ and $E_{t}$ Values 74

G. ${ }^{44} \mathrm{Ca}-{ }^{248} \mathrm{Cm}$ System $\quad 74$

1. Isotopic Distributions 75

2. Excitation Functions 75

a. Excitation Functions for Bk Isotopes 75

b. Excitation Functions for Cf Isotopes 76

c. Excitation Functions for Es Isotopes 76

d. Excitation Functions for $\mathrm{Fm}$ Isotopes 77

e. $F_{t}$ and $E_{t}$ Values 77

H. Comparison of Various Heavy Ion- $248 \mathrm{Cm}$ Systems 78

$\begin{array}{ll}\text { Chapter III Tables } & 82\end{array}$

Chapter III Figure Captions $\quad 99$

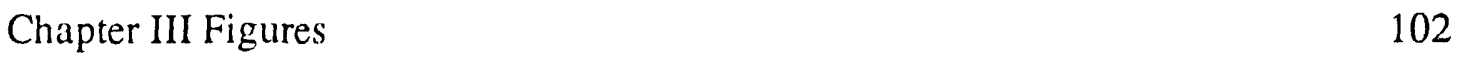

$\begin{array}{ll}\text { IV. Theory } & 129\end{array}$

$\begin{array}{ll}\text { A. Potential Energy Surfaces } & 129\end{array}$

B. Two-Step Transfer Model 132

C. Gregorich's Model of Heavy Ion Damped Reactions 135

D. Compound Nucleus Calculations: ALICE Code 137

E. Partial Wave Calculations 138

1. Sikkeland's Theory of Compound Nucleus Formation 140

2. Sikkeland's Theory of Transfer Reactions 141

3. Wilczynski's Sum-Rule Model 142

4. Presentation of New Model: PWAVE Calculations 143

Chapter IV Tables 148

Chapter IV Figure Captions $\quad 152$

$\begin{array}{ll}\text { Chapter IV Figures } & 157\end{array}$ 
IV. Conclusions

Appendix A

References 


\section{LIST OF FIGURES}

I.1 Heavy ion reaction mechanism diagram 15

I.2 Representation of the chart of the nuclides 16

$\begin{array}{lll}\text { II.1 Target system } & 47\end{array}$

II.2 Catcher foil collection efficiency calculations 48

II.3 Angular dependance of catcher foil collection efficiency 49

II.4 Cu catcher foil ch'mical procedure (Part I) 50

II.5 Cu catcher foil chemical procedure (Part II) 51

II.ó Au catcher foil chemical procedure (Short irradiations) 52

II.7 Au catcher foil chemical procedure (Long irradiations) 53

II. 8 Automated Chromatographic Chemical Element Separator System (ACCESS) $\quad 54$

II.9 ACCESS setup for Md experiments 55

II.10 Merry-Go-Round Realtime Data Acquisition and Graphics System (MG-RAGS)

II.11 MG wheel and detector arrangement 57

II.12 Alpha-gamma counting chamber 58

II.13 Cf fraction alpha spectrum $\quad 59$

$\begin{array}{lll}\text { II.14 Bk fraction gamma spectrum } & 60\end{array}$

III. $1{ }^{12} \mathrm{C}-248 \mathrm{Cm}$ isotopic distributions $(84 \mathrm{MeV}) \quad 102$

III.2 $\quad{ }^{12} \mathrm{C}-248 \mathrm{Cm}$ isotopic distributions (71 MeV) 103

III.3 Bk excitation functions from the ${ }^{12} \mathrm{C}-248 \mathrm{Cm}$ system $\quad 104$

III.4 Cf excitation functions from the ${ }^{12} \mathrm{C}-248 \mathrm{Cm}$ system $\quad 105$

III.5 Es excitation functions from the ${ }^{12} \mathrm{C}-248 \mathrm{Cm}$ system 106

III.6 Fm excitation functions from the ${ }^{12} \mathrm{C}-248 \mathrm{Cm}$ system 107

III.7 $\quad 31$ P. $248 \mathrm{Cm}$ isotopic distributions (207 MeV) $\quad 108$ 
III.8 Bk excitation functions from the ${ }^{31} \mathrm{P}-248 \mathrm{Cm}$ system 109

III.9 Cf excitation functions from the ${ }^{31} \mathrm{P}_{-} 248 \mathrm{Cm}$ system 110

III.10 Es excitation functions from the ${ }^{31} \mathrm{P}-248 \mathrm{Cm}$ system 111

III.11 Fm excitation functions from the ${ }^{31} \mathrm{P}_{-} 248 \mathrm{Cm}$ system $\quad 112$

III.12 $\quad{ }^{40} \mathrm{Ar}-248 \mathrm{Cm}$ isotopic distributions $(245 \mathrm{MeV}) \quad 113$

III.13 Bk excitation functions from the ${ }^{40} \mathrm{Ar}-{ }^{248} \mathrm{Cm}$ system 114

III.14 Cf excitation functions from the ${ }^{40} \mathrm{Ar}-248 \mathrm{Cm}$ system 115

III.15 Es excitation functions from the ${ }^{40} \mathrm{Ar}-{ }^{2} 48 \mathrm{Cm}$ system 116

$\begin{array}{lll}\text { III.16 Fraction of yield going to } 250 \mathrm{Es} g\left(6^{+} \text {state }\right) & 117\end{array}$

III.17 Fm excitation functions from the ${ }^{40} \mathrm{Ar}-248 \mathrm{Cm}$ system 118

$\begin{array}{lll}\text { III.18 } & { }^{44} \mathrm{Ca}-248 \mathrm{Cm} \text { isotopic distributions }(275 \mathrm{MeV}) & 119\end{array}$

III.19 Bk excitation functions from the ${ }^{44} \mathrm{Ca}-248 \mathrm{Cm}$ system 120

III.20 Cf excitation functions from the ${ }^{44} \mathrm{Ca}-{ }^{248} \mathrm{Cm}$ system 121

III.21 Es excitation functions from the ${ }^{44} \mathrm{Ca} \cdot{ }^{248} \mathrm{Cm}$ system 122

III.22 Fraction of yield going to $250 \mathrm{Es} g\left(6^{+}\right.$state $) \quad 123$

III.23 Fm excitation functions from the ${ }^{44} \mathrm{Ca}-{ }^{248} \mathrm{Cm}$ system 124

III.24 Bk isotopic distributions from various heavy ion- ${ }^{248} \mathrm{Cm}$ systems $\quad 125$

$\begin{array}{lll}\text { III.25 Cf isotopic distributions from various heavy ion }-248 \mathrm{Cm} \text { systems } & 126\end{array}$

III.26 Es isotopic distributions from various heavy ion $-248 \mathrm{Cm}$ systems 127

$\begin{array}{lll}\text { III.27 Fm isotopic distributions from various heavy ion }-248 \mathrm{Cm} \text { systems } & 128\end{array}$

IV.1 Potential energy surface contour plot of the $40,44,48 \mathrm{Ca}-248 \mathrm{Cm}$ systems 157

IV.2 Potential energy surface contour plot of the ${ }^{12} \mathrm{C}-248 \mathrm{Cm}$ system 158

IV.3 Potential energy surface contour plot of the ${ }^{31} \mathrm{P} .248 \mathrm{Cm}$ system 159

IV.4 Potential energy surface contour plot of the ${ }^{40} \mathrm{Ar}-248 \mathrm{Cm}$ system 160

IV.5 Vector diagram for Two-Step Transfer Model 161 
IV.6 Comparison of Two-Step Transfer Model results with experimental data

IV.7 Calculated isotopic distributions for the ${ }^{31}$ P. $248 \mathrm{Cm}$ system using a damped reaction model $(207 \mathrm{MeV})$

IV.8 Calculated isotopic distributions for the $40 \mathrm{Ar}-248 \mathrm{Cm}$ system using a damped reaction model $(245 \mathrm{MeV})$

IV.9 Calculated isotopic distributions for the ${ }^{44} \mathrm{Ca}-248 \mathrm{Cm}$ system using a damped reaction model $(275 \mathrm{MeV})$

IV.10 Calculated isotopic distributions for the ${ }^{44} \mathrm{Ca}-248 \mathrm{Cm}$ system using a damped reaction model $(236 \mathrm{MeV})$

IV.11 ALICE code salculations for the ${ }^{12} \mathrm{C}-248 \mathrm{Cm}$ system $(71 \mathrm{MeV}) \quad 167$

IV.12 ALICE code calculations for the ${ }^{12} \mathrm{C}-248 \mathrm{Cm}$ system $(84 \mathrm{MeV}) \quad 168$

IV.13 Calculated cross sections for the ${ }^{12} \mathrm{C}-248 \mathrm{Cm}$ system using the Sum-Rule Model ( $84 \mathrm{MeV}$ )

IV.14 Calculated cross sections for the ${ }^{12} \mathrm{C}-248 \mathrm{Cm}$ system using the Sum-Rule Model (84 MeV)

IV.15 Calculated cross sections for the ${ }^{40} \mathrm{Ar}-248 \mathrm{Cm}$ system using the Sum-Rule Model (245 MeV)

IV.16 Calculated cross sections for the ${ }^{40} \mathrm{Ar}-248 \mathrm{Cm}$ system using the Sum-Rule Model (245 MeV)

IV.17 Calculated cross sections for the ${ }^{44} \mathrm{Ca}-{ }^{248} \mathrm{Cm}$ system using the model developed by Hoffman and Hoffman (275 MeV)

IV.18 Calculated cross sections for the ${ }^{44} \mathrm{Ca}-{ }^{248} \mathrm{Cm}$ system using the model developed by Hoffman and Hoffman (275 MeV)

IV.19 Calculated cross sections for the ${ }^{40} \mathrm{Ar}-{ }^{248} \mathrm{Cm}$ system using the model developed by Hoffman and Hoffman (245 MeV)

IV.20 Calculated cross sections for the 31 P. $248 \mathrm{Cm}$ system using the model developed by Hoffman and Hoffman (207 MeV)

IV.21 Calculated cross sections for the ${ }^{12} \mathrm{C}-248 \mathrm{Cm}$ system using the model developed by Hoffman and Hoffman (71 MeV) 


\section{LIST OF TABLES}

I.1 Reaction parameters for various heavy ion $-248 \mathrm{Cm}$ systems

II.1 Experimental parameters $\quad 36$

II.2 Range/recoil data for catcher foils 37

$\begin{array}{lll}\text { II. } 3 & \text { Experimental conditions } & 38\end{array}$

II.4 Composition of HAVAR 39

II.5 Ionic radii of +3 actinides $\quad 40$

II.6 Experimental conditions for Md experiments 41

II.7 Decay properties of selected alpha emitters 42

II.8 Decay properties of selected spontaneously fissioning nuclides 43

II.9 Decay properties of selected nuclides which decay by electron capture or beta emission

III.1 Cross sections from the ${ }^{12} \mathrm{C}-248 \mathrm{Cm}$ system 82

III.2 Md and No cross sections from the ${ }^{12} \mathrm{C}-248 \mathrm{Cm}$ system 83

III. 3 Cross sections from the ${ }^{31}$ P. $248 \mathrm{Cm}$ system 84

III.4 Cross sections from the ${ }^{40} \mathrm{Ar}-248 \mathrm{Cm}$ system 85

III.5 Cross sections from the ${ }^{44} \mathrm{Ca}-248 \mathrm{Cm}$ system 86

$\begin{array}{lll}\text { III.6 } & \text { Excitation energies for Bk isotopes } & 87\end{array}$

III.7 Excitation energies for Cf isotopes $\quad 88$

III.8 Excitation energies for Es isotopes $\quad 89$

III.9 Excitation energies for Fm isotopes 90

III.10 Excitation energies for Md isotopes $\quad 91$

III.11 Fraction of energy transferred to heavy product for the ${ }^{12} \mathrm{C}-248 \mathrm{Cm}$
system

III.12 Fraction of energy transferred to heavy product for the ${ }^{31} \mathrm{P}-248 \mathrm{Cm}$ system 
III.13 Fraction of energy transferred to heavy product for the ${ }^{40} \mathrm{Ar}-248 \mathrm{Cm}$ system

III.14 Fraction of energy transferred to hedvy product for the ${ }^{44} \mathrm{Ca}-248 \mathrm{Cm}$ system

III.15 Fraction of yield going to ${ }^{250} \mathrm{Es} g$ from the ${ }^{40} \mathrm{Ar}$ and ${ }^{44} \mathrm{Ca}$ systems 96

III.16 Isotopic distribution maxima for various heavy ion- $248 \mathrm{Cm}$ systems

III.17 Full-widths-at-half-maximum of isotopic distributions from various heavy ion $-248 \mathrm{Cm}$ systems

IV.1 Expermental and calculated cross sections for No and Md products of the ${ }^{12,13} \mathrm{C}-249 \mathrm{Cf}$ systems.

IV.2 Calculated $\Gamma_{n} /\left(\Gamma_{n}+\Gamma_{f}\right)$ values for various actinides

IV.3 Excitation energies for products of the ${ }^{44} \mathrm{Ca}-248 \mathrm{Cm}$ system 150

IV.4 Excitation energies for products of the ${ }^{12} \mathrm{C}-248 \mathrm{Cm}$ system 


\section{INTRODUCTION}

\section{A. Heavy Ion Reactions}

\section{General Features}

Nuclear reactions can generally be described as those interactions between two nuclei, a nucleus and an elementary particle, or a nucleus and a photon in which a change in the nucleus takes place [FRI81]. The results of such interactions are numerous and are an active area of research in the field of nuclear science. However, nuclear reactions between heavy ions $(\mathrm{HI})$ are especially important due to their unique characteristics. In particular, heavy ions have large nuclear radii, high nuclear charges and angular momenta, and small de Broglie wavelengths (defined below) [SCO77]. Since the reduced de Broglie wavelength is small compared to the internuclear distances involved in these types of reactions, heavy ion reactions can be treated in a classical manner [EIS75] [GLE83].

Presently, there are only 270 known stable nuclides. Luring the last seventy years, approximately 1300 radioisotopes have been discovered [GEN89] [SCO77]. However, it has been predicted that 6000 different nuclides could be formed from the interactions of $2 \mathrm{GeV} \mathrm{U}+\mathrm{U}$ ions [SCO77]. Hence, heavy ion reactions have and will be an important tool in uncovering the secrets of nuclear structure and stability.

\section{Classical Mechanics: An Introduction}

A schematic diagram of a heavy ion projectile impiriging on a target nucleus is shown in FIG. 1.1. This figure illustrates, among other things, the impact parameter, $b_{m}$, and the scattering angle, $\theta$.

Thu Coulomb barrier between t:wo nuclei is the center-of-mass energy required to bring the two from infinite separation to a touching sphere configuration, and is given by 


$$
V_{c}=-\frac{z_{1} z_{2} e^{2}}{R_{1}+R_{2}},
$$

where $Z_{1}$ and $Z_{2}$ are the atomic numbers of the two nuclei, $e$ is the elementary charge, and $R_{1}$ and $R_{2}$ are the respective nuclear radii. The distance of closest approach, $d$, for a head-on collision between these two nuciei is then

$$
d=\frac{\bar{z}_{1} z_{2} e^{2}}{E_{c m}},
$$

where $E_{c m}$ is the center of mass kinetic energy. The reduced de Broglie wavelength, $\lambda$, for a nucleus is

$$
\lambda=\frac{\hbar}{m v},
$$

where $\hbar$ is $h / 2 \pi, h$ is Planck's constant, $m$ is the mass of the heavy ion, and $v$ is the relative velocity of the ion. Hence, the Sommerfeld parameter, $\eta$, is used for these situations and is defined as

$$
\eta=\frac{Z_{1} Z_{2} e^{2}}{\hbar v}
$$

This parameter is one half the ratio of the distance of closest approach to the reduced de Broglie wavelength. Once again, for the classical domain to be applicable, $\eta>>1$. The Sommerfeld parameters have been calculated for ${ }^{12} \mathrm{C}, 31 \mathrm{P},{ }^{40} \mathrm{Ar}$, and ${ }^{44} \mathrm{Ca}$ with ${ }^{248} \mathrm{Cm}$ and are shown in Table I.1. These calculations were done at the highest bombarding energy for each projectile. 
For large $\eta$, the angular distributions for the projectile-like fragments have their maxima near the grazing angle [GAL76]. The grazing angle in the center-of-mass system, $\theta_{g r}$, can be calculated by

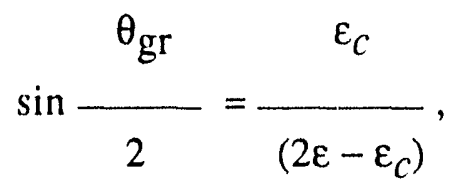

where $\varepsilon_{c}=V_{c} / \mu, \mu$ is the reduced mass of the system, and $\varepsilon$ is the relative kinetic energy [MCF82] [SCO77]. Since the grazing angle can only be calculated at energies above the Coulomb barrier, the quarter-point angle from the Fresnel scattering model [FRA78] [SCO77] should be used for near- and below-Coulomb barrier interactions. The quarterpoint angle, $\theta_{1 / 4}$, in the center-of-mass system is

$$
\theta_{1 / 4}=2 \sin ^{-1} \frac{\eta}{k R_{\text {exp }}-\eta}
$$

where $\eta$ is the Sommerfeld parameter, $k$ is the asymptotic wave number, and $R_{\text {exp }}$ is the experimentally determined interaction radius [WIL80]. The Fresnel model assumes there is a well-defined interaction radius, $R_{\text {exp }}$, for each target-projectile combination, that separates elastic scattering events and nuclear reactions in configuration space.

Using the sharp cutoff approximation, assuming a grazing collision with no mass transfer, and a loss of kinetic energy of $10 \mathrm{MeV}$, the scattering angles of the projectilelike and target-like products were calculated. The velocity of the center-of-mass of a dinuclear system in the laboratory frame of reference is 


$$
V_{s}=1.389 \frac{E_{p}^{1 / 2}}{m_{p}^{1 / 2}} \frac{m_{p}}{m_{t}+m_{p}} \mathrm{~cm} / \mathrm{ns},
$$

where $E_{p}$ is the lab frame kinetic energy of the projectile, $m_{p}$ is the mass of the projectile, and $m_{t}$ is the mass of the target [TÜR89]. The center-of-mass velocity of the target-like fragment is given by

$$
V_{t}=1.389 \frac{\left(\text { TKEm }_{p}\right)^{1 / 2}}{\left[m_{t}\left(m_{t}+m_{p}\right)\right]^{1 / 2}} \mathrm{~cm} / \mathrm{ns},
$$

where $T K E$ is the total kinetic energy of the fragments and was assumed to be $10 \mathrm{MeV}$ less than the center-of-mass energy of the projectile. The center-of-mass velocity of the projectile-like fragment is

$$
V_{p}=1.389 \frac{\left(T K E m_{t}\right)^{1 / 2}}{\left[m_{p}\left(m_{t}+m_{p}\right)\right]^{1 / 2}} \mathrm{~cm} / \mathrm{ns}
$$

The scattering angle of the projectile-like fragment in the laboratory system is then

$$
\theta_{p r o j}=\arctan \frac{V_{p} \sin \left(\theta_{c m}\right)}{V_{p} \cos \left(\theta_{c m}\right)+V_{s}},
$$

where $\theta_{\mathrm{cm}}$ is the angle between the nuclei in the entrance channel and exit channel. The scattering angle of the target-like fragment in the laboratory system is given by 


$$
\theta_{\text {proj }}=\arctan \frac{V_{t} \sin \left(\theta_{c m}\right)}{V_{t} \cos \left(180^{\circ}-\theta_{c m}\right)+V_{s}} .
$$

Table I. 1 lists $\theta_{\text {proj }}$ and $\theta_{\text {targ }}$ for the four systems investigated.

The particular type of reaction that results from an interaction between two heavy nuclei depends upon the impact parameter of the projectile, the kinetic energy of the projectile, and the masses of both the target and projectile [FRI81]. Since both nuclei are positively charged, the Coulomb barrier must be surmounted before the two will actually come in contact with each other. This can be achieved by giving the projectile sufficient kinetic energy.

\section{Elastic and Inelastic Scattering}

At large impact parameters where the separation distance $R_{\text {sep }}$ is greater than the interaction radius $R_{i n t}\left(R_{i n t}=R_{p r o j}+R_{\text {target }}\right)$, a process known as elastic scattering takes place. In this process the total kinetic energy of the system (projectile and target) is conserved. Heavy icns provide valuable information about interaction radii via elastic scattering. Another important type of process occurring at large impact parameters is termed inelastic scattering. In this process, the target nucleus is left in an excited state at the expense of the kinetic energy of the projectile. Extremely high-spin states can be induced in target nuclei by heavy ion projectiles through this type of process.

\section{Transfer Reactions}

When $R_{\text {sep }}$ is approximately equal to $R_{i n t}$, the overlap of nuclear matter takes place, exchange of nucleons can occur, and the angular distributions of the projectile-like products will peak near the grazing angle. During this process, the projectile approaches the target, the two come into contact forming an intermediate complex with a slight overlap between the two nuclei. After exchanging energy, angular momentum, and nucleons, the intermediate th 2 quickly separates into two new nuclei, a projectile-like fragment and a target-like fragment. Due to the binary nature and large impact 
parameters of transfer reactions, the projectile-like fragment can carry off a large fraction of the kinetic energy and angular momentum, thus leaving the target-like product with little excitation energy. The contact time is on the order of $10^{-22}$ seconds [TÜR89]. Since the interaction times for these types of reactions are so short, little equilibration occurs; hence the neutron-to-proton ratio, angular momentum, and kinetic energy remain essentially unequilibrated.

\section{Deeply Inelastic Reactions}

If $R_{\text {sep }}<R_{\text {int }}$, significant overlap of nuclear matter occurs, large numbers of nucleons, excitation energy, and angular momentum can be exchanged between the two nuclei, and the type of interaction is termed deeply inelastic. The intermediate complex formed between the target and projectile will rotate because of the amount of angular momentum introduced by the projectile. A large fraction of the projectile kinetic energy is transformed into internal excitation so that when the intermediate separates, two new products with a great deal of excitation energy are formed. The interaction times for deeply inelastic reactions are on the order of $10^{-21} \mathrm{~s}$, sufficiently long to enable the neutron-to-proton ratio to equilibrate. There is heavy damping of the kinetic energy and some equilibration of orbital angular momentum [DOS85] [GRE85] [KRA77] [MOR81] [NÖR75] [RIE79] [SCH77].

\section{Complete Fusion Reactions}

At even smaller impact parameters, a projectile nucleus can totally amalgamate with a target nucleus resulting in a compound nucleus. During these reactions the identities of the target and projectile are lost and equilibration of the neutron-to-proton ratio, kinetic energy, and angular momentum is achieved. In some instances, however, p eequilibrium emission of particles can occur.

\section{B. A Brief History of Transfer Reactions}

Multinucleon transfer reactions were first investigated in detail by Kaufmann and Wolfgang in the late 50's and early 60)'s [KAU59] [KAU60]. Studying interactions of 
$16 \mathrm{O}$ projectiles with $\mathrm{Al}, \mathrm{Cu}$, and $\mathrm{Sn}$ targets, they found that the $(p 2 n)$ and $(2 p 3 n)$ cross sections were larger than expected in comparison to single nucleon transfer reactions. These results contradicted those expected from transfer reactions involving a Coulomb barrier tunneling mechanism, leading them to propose the "contact transfer model." This model involved the interactions between a target and projectile at distances approximately equal to the radius of the projectile. Under these conditions a dumbbellshaped intermediate could be formed, allowing nucleons to be exchanged. Because the Coulomb and centripetal forces were greater than the nuclear binding force, the intermediate would break up into a projectile-like fragment and target-like fragment before it had rotated one half of a revolution [KAU60].

Hahn et al. [HAH74] produced $245,244 \mathrm{Cf}$ via the ${ }^{239} \mathrm{Pu}\left({ }^{12} \mathrm{C}, \alpha x n\right),{ }^{235} \mathrm{U}\left({ }^{16} \mathrm{O}, \alpha x n\right)$, ${ }^{237} \mathrm{~Np}\left({ }^{14} \mathrm{~N}, \alpha x n\right),{ }^{242} \mathrm{Pu}\left({ }^{12} \mathrm{C}, \alpha(x+3) n\right)$ and ${ }^{238} \mathrm{U}\left({ }^{12} \mathrm{C}, 5 n-6 n\right)$ reactions. Based upon these data they made the following observations:

1. The probability of transferring a cluster of nucleons decreases as the size of the cluster increases. Viewing these reactions as the division of the projectile into an alpha particle and a residual cluster, they argued that it was logical that the alpha cluster nuclei such as ${ }^{12} \mathrm{C}$ and ${ }^{16} \mathrm{O}$ have larger yields than ${ }^{14} \mathrm{~N}$.

2. The cross sections for the $\left({ }^{12} \mathrm{C}, \alpha 2 n\right)$ and $\left({ }^{12} \mathrm{C}, \alpha 3 n\right)$ channels were larger than the $\left({ }^{12} \mathrm{C}, \alpha 5 n\right)$ and $\left({ }^{12} \mathrm{C}, \alpha 6 n\right)$ channels. This signified to the authors that fission was effectively competing with the evaporation of more than two or three neutrons.

Focusing on the ${ }^{238} \mathrm{U}\left({ }^{12} \mathrm{C}, 5 n-6 n\right)$ and ${ }^{239} \mathrm{Pu}\left({ }^{12} \mathrm{C}, \alpha 2 n-\alpha 3 n\right)$ channels, Hahn et al. performed a detailed comparison in which they measured excitation functions, recoil ranges, and angular distributions of the products. The data from the $\mathrm{Cf}$ isotopes produced by the ${ }^{12} \mathrm{C}-238 \mathrm{U}$ system were consistent with a compound nucleus mechanism while the data from the same $\mathrm{Cf}$ isotopes produced in the ${ }^{12} \mathrm{C}-23{ }^{2} \mathrm{Pu}$ system were consistent with a transfer mechanism. The compound nucleus products displayed forward-peaked angular distributions in the laboratory frame of reference while the 
transfer reaction products had angular distributions that peaked at $\cong 17^{\circ}$ (at energies of 1.06 and $1.17 \mathrm{X}$ Coulomb barrier). Based upon these results, Hahn et al. made use of a semi-classical, two step transfer reaction model involving the transfer of a cluster of nucleons in the first step and the subsequent Rutherford scattering of the projectile-like fragment in the second step.

Eskola et al. [ESK70] [ESK71] [ESK73] [ESK75] produced a variety of Es, Md, No, and $\mathrm{Lr}$ isotopes via $(H I, x n),(H I, \alpha \times n)$, and $(H I, 2 \alpha x n)$ reactions on actinide targets. They found the production cross sections for the $\left({ }^{12} \mathrm{C}, \alpha 2 n\right)$ and $\left({ }^{12} \mathrm{C}, \alpha 3 n\right)$ channels were higher than the cross sections for the $\left({ }^{12} \mathrm{C}, 4 n\right)$ and $\left({ }^{12} \mathrm{C}, 5 n\right)$ channels. They viewed the $\left({ }^{12} \mathrm{C}, \alpha 2 n\right)$ channel as a carbon projectile breaking up into a ${ }^{8} \mathrm{Be}$ nucleus and an $\alpha$ particle. In addition, the $\left({ }^{12} \mathrm{C}, \alpha \times n\right)$ channels had higher production cross sections than the $\left({ }^{13} \mathrm{C}, \alpha \times n\right)$ reaction channels. This artifact was attributed to $\alpha$ clustering in the ${ }^{12} \mathrm{C}$ nucleus. Eskola [ESK75] also found that cross sections for products with $Z_{\text {target }}<$ $Z_{\text {product }}<Z_{\text {target }}+$ projectile were larger than the cross sections for compound nucleus products.

The fact that transfer reactions can produce target-like fragments with low excitation energy becomes advantageous in certain areas of the periodic table. Specifically, the production of "cold" target-like products becomes important in the actinide region where the fission barriers are on the order of 5-6 MeV [BRI79] [BRI80]. With low excitation energies, actinide products of transfer reactions have a greatly reduced probability of fission and/or particle emission during their deexcitation [HOF86]. The bombardment of actinide targets with heavy ion projectiles can effectively be used to produce a great variety of actinide products via transfer reactions. In particular, by choosing neutron-rich projectiles and/or targets, neutron-rich actinide products can be produced with easily detectable cross sections. By producing new, neutron-rich actinides, the so called "island of stability" or "superheavy island" (see FIG I.2) can possibly be reached. It is postulated that this island exists at the next closed proton and neutron shells of 114 and 184 , 
respectively [HER79].

\section{Transfer Reaction Studies Using ${ }^{248} \mathrm{Cm}$ as a Target}

Because of the availability of relatively large quantities of $248 \mathrm{Cm}$, this $\mathrm{n}$. Lide has become a popular target material for many different transfer reaction studies. ${ }^{248} \mathrm{Cm}$ is an attractive target material for many reasons including its high neutron-to-proton ratio $(N / Z=1.583)$, long half-life $\left(t_{1} / 2=3.40 \times 10^{5} \mathrm{y}\right)$, and its availability in nearly isotopically pure milligram quantities [HOF85].

Numerous actinide prolluction studies using $248 \mathrm{Cm}$ targets with various projectiles, ranging from ${ }^{13} \mathrm{C}$ to $238 \mathrm{U}$, have been performed in order to gain some insight into the reaction mechanism(s) involved [ESK75] [GÄG86] [HOF85A] [LEE82] [LEE83] [MOO86] [SCH82] [TÜR89] [WEL87].

In an attempt to study the influence of an additional pair of neutrons in the projectile on the actinide production cross sections, Lee et al. [LEE82] bombarded ${ }^{248} \mathrm{Cm}$ with the projectile pairs $16,18 \mathrm{O}$ and $20,22 \mathrm{Ne}$. It was found that as the number of neutrons in the projectile was increased by two, the corresponding maxima of the mass-yield curves (plots of cross section vs. mass number for a given $Z$ ) for the above target elements $\left(Z_{\text {product }}>Z_{\text {target }}\right)$ were increased by about two mass units. Hence, the neutron-rich projectile of each pair enhanced the neutron-rich actinide production.

Experiments using the projectiles $40,48 \mathrm{Ca}$ on $248 \mathrm{Cm}$ targets were performed to see what the effect of the eight neutron excess of ${ }^{48} \mathrm{Ca}$ relative to ${ }^{40} \mathrm{Ca}$ would have on the final actinide production distribution [HOF85A]. Shifts to heavier masses of only 2-3 units in the mass-yield curves for the elements Bk, Cf, Es, and Fm were observed in the ${ }^{48} \mathrm{Ca}$ system relative to the ${ }^{40} \mathrm{Ca}$ system. The eight neutron excess of ${ }^{48} \mathrm{Ca}$ relative to ${ }^{40} \mathrm{Ca}$ did not increase the maxima of the isotopic distributions by eight mass units and hence had only a partial effect on the final isotopic distributions of the above target products.

Welch et al. [WEL85] [WEL87] observed in the $129,132,136 \times \mathrm{Xe}^{248} \mathrm{Cm}$ systems that 
the peaks in the isotopic distributions for $\mathrm{Fm}, \mathrm{Bk}$, and Pu shifted to higher mass numbers as the projectile mass number increased. Shifts in the Cf and Es peaks could not be verified because production cross sections on the neutron-deficient side of the mass yield curves were not measured. However, production cross sections for the most neutron-rich above target nuclides were found to be independent of the projectile mass. Potential energy surfaces comparing the energies of the system of two touching spheres before and after the transfer of neutrons and protons were calculated and compared with the reaction cross sections. The potential energy surfaces were unable to succes:fully predict the maxima of the mass-yield curves, suggesting to the authors that deeply inelastic reactions may not be the dominant reaction mechanism.

Schädel et al. [SCH78] [SCH82] studied the ${ }^{238} \mathrm{U}-248 \mathrm{Cm}$ system in addition to the $238 \mathrm{U}-238 \mathrm{U}$ system. Upon comparing various heavy ion $-248 \mathrm{Cm}$ systems, they noted shifts in the centroids of the isotopic distributions of the actinide products. However, much to their surprise, they noted that the "nearby" $\left(Z_{\text {product }} \cong Z_{\text {target }}\right)$ actinides produced from the ${ }^{248} \mathrm{Cm}$ targets interacting with $16,18 \mathrm{O},{ }^{20}, 22 \mathrm{Ne},{ }^{48} \mathrm{Ca},{ }^{136} \mathrm{Xe}$, and $238 \mathrm{U}$ had peak yields that were all the same order of magnitude.

Since transfer reactions involving actinide targets provide a potential means of producing new neutron-rich actinides, a systematic study of these processes was continued as a primary goal of this thesis. Since ${ }^{24} \mathrm{Cm}$ had been used as a target in many previous transfer reaction studies, it seemed logical to extend the systematic study of transfer reactions using this nuclide as the target material. With the addition of data from new target-projectile combinations, it was hoped that the present understanding of transfer reactions could be broadened and theoretical models developed which could accurately model the reaction mechanism involved, and provide predictions for unmeasured systems.

\section{Rationale Behind Projectile Selection}

The first projectile chosen for this study, ${ }^{44} \mathrm{Ca}$, was used in order to further investigate 
the effect of the neutron number of the projectile on the final isotopic distributions in transfer reactions involving ${ }^{248} \mathrm{Cm}$ targets. Since previous studies had used the ${ }^{40} \mathrm{Ca}$ ${ }^{248} \mathrm{Cm}$ and ${ }^{48} \mathrm{Ca}-{ }^{248} \mathrm{Cm}$ systems, ${ }^{44} \mathrm{Ca}$ was an ideal candidate. ${ }^{44} \mathrm{Ca}$ has a neutron number of 24 , which is between the neutron numbers of 20 and 24 , respectively of the doubly magic nuclei $4 \mathrm{C}_{\mathrm{Ca}}$ and ${ }^{48} \mathrm{Ca}$. Since both ${ }^{40} \mathrm{Ca}$ and ${ }^{48} \mathrm{Ca}$ are doubly magic, ${ }^{44} \mathrm{Ca}$ could be used to study the influence of neutron shell effects on the final isotopic distributions of the actinide products. This stable nuclide has a natural abundance of 2.086 atom \% [WAL84] and $600 \mathrm{mg}$ was obtained from Oak Ridge National Laboratory as a separated isotope (98.6 atom \%) in the metallic form. In addition, reasonably intense beamı $(\cong 100 \mathrm{PnA}$ ) could be obtained from the 88-Inch Cyclotron at Lawrence Berkeley Laboratory.

The ${ }^{40} \mathrm{Ar}-248 \mathrm{Cm}$ system was studied next because the neutron-to-proton ratio of ${ }^{40} \mathrm{Ar}(1.22)$ is quite similar to the 1.20 ratio of $44 \mathrm{Ca}$. In addition, $40 \mathrm{Ar}$ has the same mass number as the doubly magic ${ }^{40} \mathrm{Ca}$. Since ${ }^{40} \mathrm{Ar}$ is a stable isotope of high natural abundance (99.600 atom \%) [WAL84], it is readily available. It is also easily accelerated with high intensity by the 88 -Inch Cyclotron.

$31 \mathrm{P}$ was chosen as a projectile for two reasons. First of all it has an odd number of protons, and until this study, no detailed transfer reaction studies in the actinide region had been performed using projectiles with odd $Z$. The odd $Z$ projectile was used in order to see if any projectile pairing effects could be observed in the final isotopic distributions. Secondly, ${ }^{31} \mathrm{P}$ has a neutron-to-proton ratio of 1.07 , similar to the ratios of other projectiles used in previous studies. Since $31 \mathrm{P}$ has a natural abundance of 100 atom \% [WAL84], it was rather easily obtainable. Red phosphorus was used in the ion source at the 88 -Inch Cyclotron.

The final projectile used in this study was ${ }^{12} \mathrm{C}$. This projectile was chosen because it has a neutron-to-proton ratio of 1.00 , exactly the same as ${ }^{40} \mathrm{Ca}$. Since the lightest projectile used in a detailed transfer reaction study on $248 \mathrm{Cm}$ was $16 \mathrm{O}$, the extension of 
this study down to an even lighter element was desirable to see if any mechanistic changes occur. ${ }^{12} \mathrm{C}$ can be thought of as simply a cluster of three $\alpha$ particles. Hence, the $\left({ }^{12} \mathrm{C}, \alpha x n\right)$ and $\left({ }^{12} \mathrm{C}, 2 \alpha x n\right)$ reaction channels might be enhanced. Additionally, ${ }^{12} \mathrm{C}$ is easily obtainable in gaseous forms such as $\mathrm{CH}_{4}$ or $\mathrm{CO}_{2}$ winch make ideal sources for the 88-Inch Cyclotron ECR ion source. 
Table 1.1. Calculated Sommerfeld parameter, $\eta$, for ${ }^{12} \mathrm{C},{ }^{31} \mathrm{P},{ }^{40} \mathrm{Ar}$, and ${ }^{44} \mathrm{Ca}$ with ${ }^{248} \mathrm{Cm}$.

\begin{tabular}{lrrrrl}
\hline \multicolumn{5}{c}{ Bombarding } \\
Projectile & $\begin{array}{c}V_{c} \\
(\mathrm{MeV})\end{array}$ & $\begin{array}{c}\text { Energy } \\
(\mathrm{MeV})\end{array}$ & $\eta$ & $\begin{array}{c}\theta_{\text {proj }} \\
\text { (degrees) }\end{array}$ & $\begin{array}{l}\theta_{\text {targ }} \\
\text { (degrees) }\end{array}$ \\
\hline${ }^{12} \mathrm{C}$ & 68.6 & 93 & 32 & 66 & 53 \\
$31 \mathrm{P}$ & 156.1 & 21 & 282 & 64 & 54 \\
$40 \mathrm{Ar}$ & 181.9 & 246 & 102 & 64 & 53 \\
${ }^{44} \mathrm{Ca}$ & 199.8 & 271 & 112 & 63 & 53 \\
\hline
\end{tabular}




\section{Figure Captions}

Figure I.1. Schematic diagram of a heavy ion projectile $\left(A_{1}, Z_{1}\right)$ impinging upon a stationary target nucleus $\left(A_{2}, Z_{2}\right) . b_{m}$ is the impact parameter, $\theta$ is the scattering angle, $R_{1}$ is the projectile nucleus radius, and $R_{2}$ is the target nucleus radius.

Figure 1.2. Schematic representition of the chart of the nuclides taken from [GRE89]. 


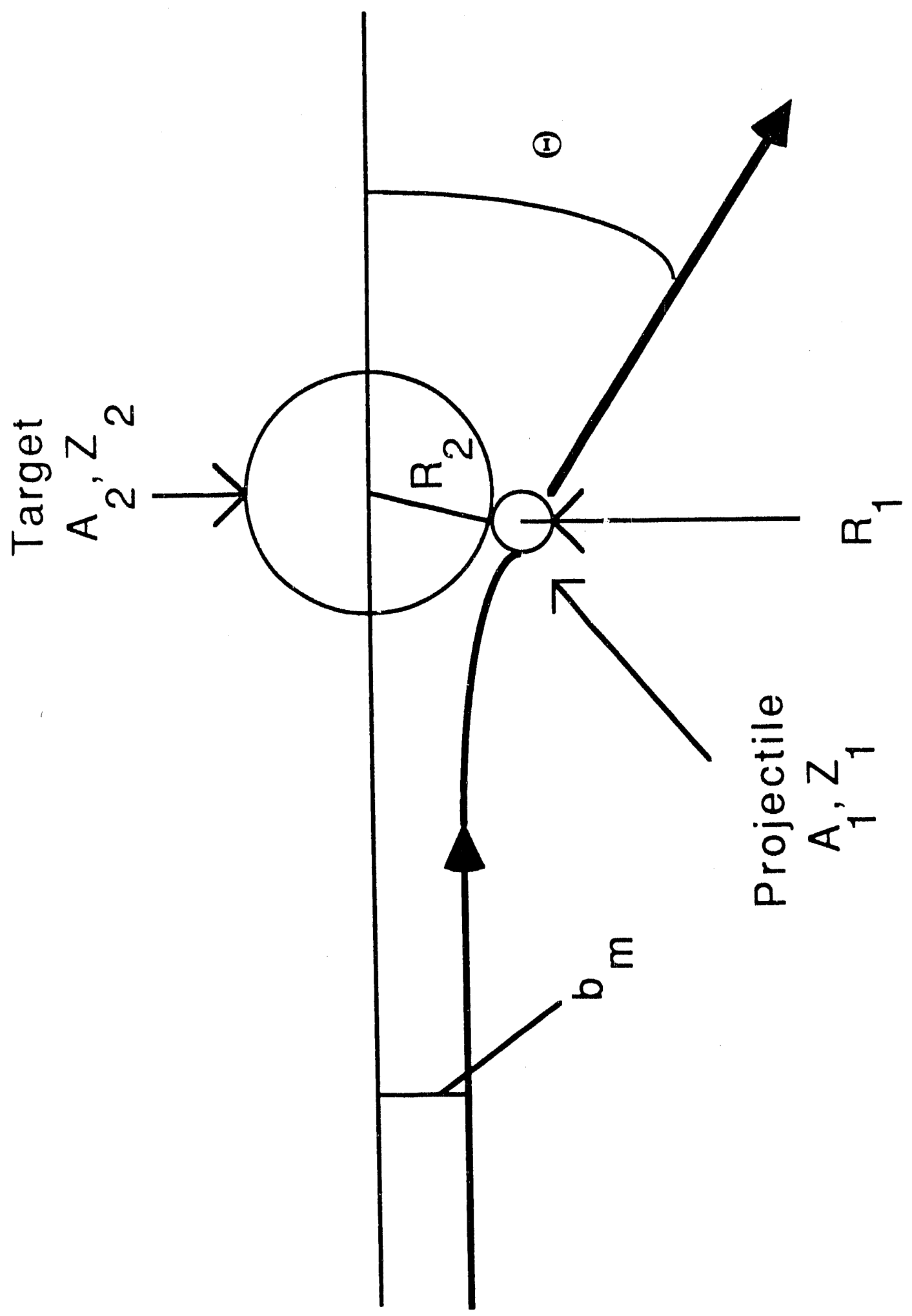

Fig. I.1 


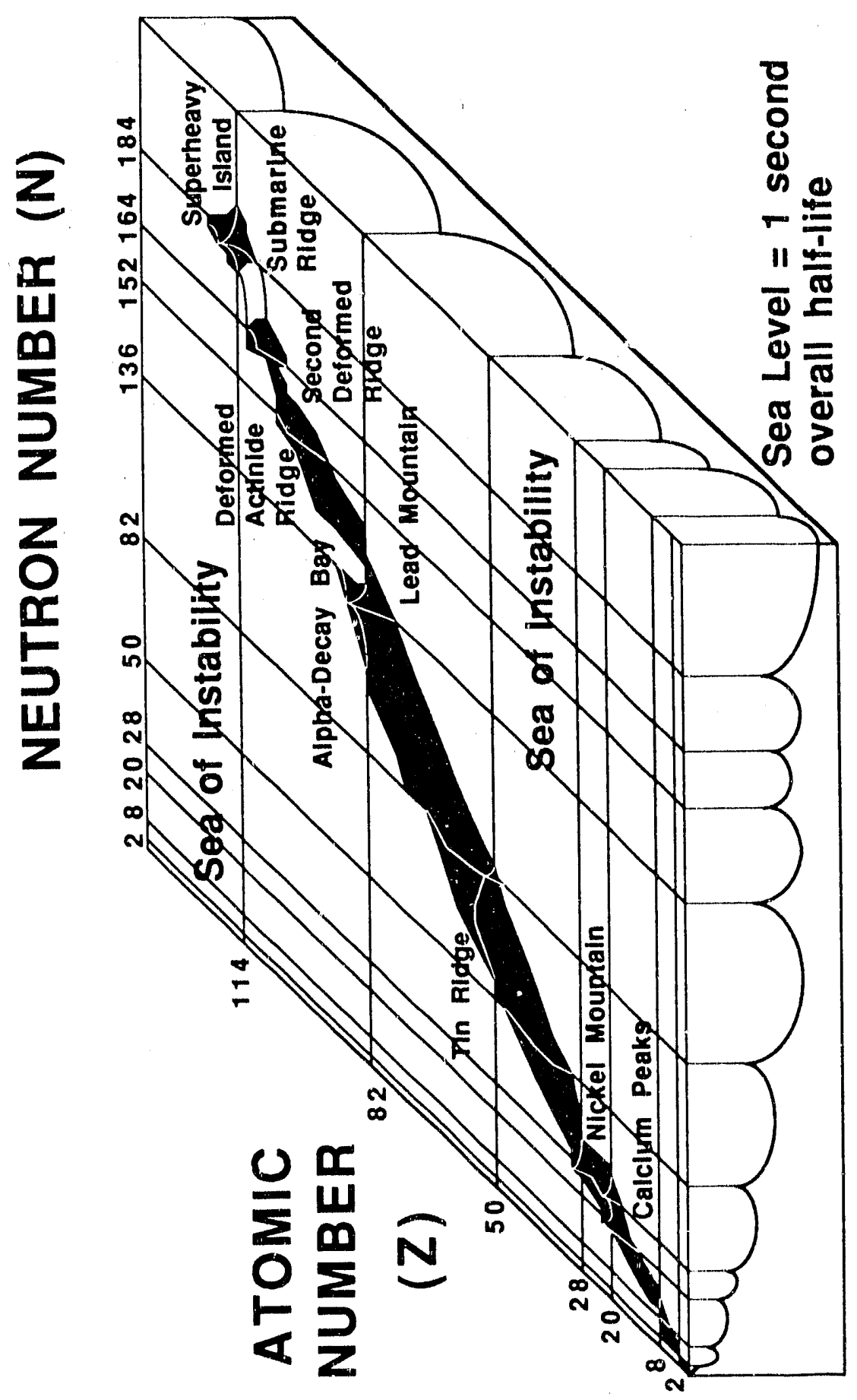

Fig. 1.2 


\section{EXPERIMENTAL}

\section{A. Target Requirements}

Very strict guidelines are required of targets used in heavy ion bombardments at particle accelerators [HOF88]. When measuring reaction cross sections, the target must be thin and uniform so the energy of the bombarding particles is well defined upon passage through the target material. In addition, the target material must also be thin enough so that the recoiling target-like fragments can escape from the target material if a catcher foil technique is used.

The passage of a heavy ion beam through an actinide target produces a large amount of heat and extreme ionizing conditions. The target and its backing must therefore be able to withstand such conditions. For these reasons $\mathrm{Cm}_{2} \mathrm{O}_{3}$ (m.p. $2260 \pm 25{ }^{\circ} \mathrm{C}$ ) [BAY73] was chosen as the target material and Be metal (m.p. $1278 \pm 5^{\circ} \mathrm{C}$ ) [CRC75] as the target backing.

The ${ }^{248} \mathrm{Cm}$ targets $\left(97.44 \%{ }^{248} \mathrm{Cm}, 2.53 \%{ }^{246} \mathrm{Cm}, 0.025 \%{ }^{245} \mathrm{Cm}, 0.010 \%\right.$ ${ }^{247} \mathrm{Cm}$, and $1 \times 10^{-4 \%}{ }^{244} \mathrm{Cm}$ ) were prepared by the electrodeposition of $\mathrm{Cm}$ in a 0.6 $\mathrm{cm}$ diameter on a Be foil in stepwise fashion from an isopropanol solution [GRE85]. After depositing approximately $70 \mu \mathrm{g} / \mathrm{cm}^{2}$ of $248 \mathrm{Cm}$, the Be was placed in an oven and heated to $500{ }^{\circ} \mathrm{C}$ to convert the $\mathrm{Cm}$ to $\mathrm{Cm}_{2} \mathrm{O}_{3}$. To determine its thickness, the target was assayed at low geometry with an $\alpha$-spectrometer system utilizing a $\mathrm{Si}(\mathrm{Au})$ solid state surface barrier detector. The electrodeposition, heating, and counting were repeated until the desired target thickness was reached. Table II.1 lists the thicknesses of the targets used in the various experiments of this study.

\section{B. Target System}

All of the irradiations of the ${ }^{248} \mathrm{Cm}$ targets were performed at the Lawrence Berkeley Laboratory 88-Inch Cyclotron. Because a radioactive target was used, precautions had to be taken to insure that the 88-Inch Cyclotron would not become contaminated in the 
event of a vacuum failure. For this reason the "slammer valve" was used. Should a vacuum problem arise, the "slammer valve" would simply seal the beam line between the target system and the cyclotron, effectively isolating the cyclotron from possible contamination with ${ }^{248} \mathrm{Cm}$. The positive ion beams, after exiting the cyclotron passed through a "beam wobbler". This device is simply the stator of an induction motor used to move the beam in circles over the target area in order to spread the beam and prevent any local overheating in the target [MOO83]. From the wobbler, the positive ion beams passed through a water-cooled graphite collimater (inner diameter $6 \mathrm{~mm}$ ), a $1.8 \mathrm{mg} / \mathrm{cm}^{2}$ HAVAR isolation foil, $0.2 \mathrm{mg} / \mathrm{cm}^{2}$ nitrogen cooling gas, the $2.75 \mathrm{mg} / \mathrm{cm}^{2}$ Be target backing, and finally the $\mathrm{Cm}_{2} \mathrm{O}_{3}$ target material. The reaction products recoiling out of the target were stopped and collected in $1.6 \mathrm{~cm}$ diameter copper or gold catcher foils located approximately $0.4 \mathrm{~cm}$ from the target. The compositions and thicknesses of the catcher foils used in these experiments are listed in 'Table II.1. The accelerated ions that did not interact with the target material were stopped by a water-cooled graphite beam stop. The total electrical current deposited in the target system by the beam was measured by an integrating electrometer. The integrated beam current was recorded at regular intervals throughout all of the bombardments so that accurate beam histories could be used in the calculation of production cross sections. A schematic diagram of the target system at the 88- Inch Cyclotron is shown in Fig. II.1.

\section{Catcher Foil Collection Efficiency}

Since both the target and catcher foil have finite dimensions, the probability of a recoiling reaction product hitting the catcher foil must be considered. This problem has been treated in detail by Moody and only a brief summary will be presented here [MOO83]. If one has a circular catcher foil of radius $a$ at distance $b$ from a circular target of radius $R$, the probability of a ecoiling reaction product missing the catcher foil may easily be calculated assuming the target and catcher foil are parallel (see Fig. II.2). At any point $r$ from the axis connecting the centers of the target and catcher foil, reaction 
products are emitted at a variety of angles $\theta$ with respect to the beam axis. However, if the reaction products are emitted at angles smaller than certain angle $\theta_{\min }$, all such products are collected regardless of their orientation about the axis. Likewise, if the recoiling reaction products are emitted at angles larger than angle $\theta_{\max }$, all such products will miss the catcher foil, regardless of their orientation. If, however, the recoiling reaction products are emitted at angles between $\theta_{\min }$ and $\theta_{\max }$, a certain fraction of the products will hit the catcher foil, depending on the azimuthal angle at which the products are emitted. The probability of a recoiling reaction product missing the catcher foil when it is emitted at a distance $r$ from the axis at an angle $\theta$ (where $\theta_{\min }<\theta<\theta_{\max }$ ) is given by

$$
\mathrm{P}_{\theta, r}=\frac{1}{\pi} \sin ^{-1} \frac{b^{2} \tan ^{2} \theta-a^{2}+r^{2}}{2 r b \tan \theta}+\frac{\pi}{2} .
$$

By evaluating this expression over the entire area of the target and over all azimuthal angles, the total collection efficiency of the catcher foil can be obtained. A computer code was written (see Appendix A) to calculate the probability of a recoiling reaction product hitting the catcher foil as a function of the angle at which the recoiling nucleus was emitted (in the lab frame) [LEY90B]. The results of this calculation for the target system used in this study are plotted in Fig. II.3. From Fig. II.3 it can be seen that the probability of a recoiling product nucleus hitting the catcher foil is 1 if it is emitted between $0^{\circ}$ and $51^{\circ}$ and then decreases monotonically to 0 between $51^{\circ}$ and $71^{\circ}$.

The laboratory system scattering angles for the target-like fragments, $\theta_{\text {targ }}$, which are listed in Table II.1, indicate that the largest angles at which the target-like fragments recoil is $53-54^{\circ}$. At $54^{\circ}$, the probability of a recoiling nucleus hitting the catcher foil is 0.95. Hence, even at the highest bombarding energies, losses due to the recoiling atoms missing the catcher foil are small. 
The maximum recoil energy possible for a binary-type interaction, assuming that the reaction $Q$ value and the amount of mass transferred is small, is for a completely elastic collision at an impact parameter of zero. For $319-\mathrm{Mev}{ }^{44} \mathrm{Ca}$ ions, the recoiling energy of a target-like product is about $140 \mathrm{MeV}$. The range of $140 \mathrm{MeV} 248 \mathrm{Bk}$ ions in $\mathrm{Au}$ is $12 \mathrm{mg} / \mathrm{cm}^{2}$ [NOR70], much thinner than the $19.6 \mathrm{mg} / \mathrm{cm}^{2}$ Au catcher foils used. Maximum recoil energies for target-like products have been calculated for the other systems in the present study and are given in Table II.2 along with their associated ranges in the catcher foil material and the thicknesses of the catcher foils used. In all cases the catcher foils were sufficiently thick to stop the recoiling reaction products, assuming the reactions products hit the catcher foil, especially since most products will recoil with significantly less than the maximum possible recoil energy.

In many instances, however, the $Q$ value and/or the mass transfer for a particular reaction channel are significant and cannot be ignored, especially in the case of the ${ }^{12} \mathrm{C}$ system. Fortunately recoil range data exist on the actinide products from the $18 \mathrm{O}$ ${ }^{245} \mathrm{Cm}$ and ${ }^{18} \mathrm{O}-249 \mathrm{Cf}$ systems [MCF82]. ${ }^{246} \mathrm{Cf}$ and ${ }^{252} \mathrm{Fm}$ produced from the ${ }^{18} \mathrm{O}$ $245 \mathrm{Cm}$ reaction at $1.18 \times$ Coulomb barrier, were both found to have an average range of approximately $0.25 \mathrm{mg} / \mathrm{cm}^{2}$ in $\mathrm{Al}$. Based on range and stopping-power tables [NOR70], this corresponds to a range of roughly $0.65 \mathrm{mg} / \mathrm{cm}^{2}$ in $\mathrm{Au}$, much thinner than the 2.47 $\mathrm{mg} / \mathrm{cm}^{2}$ Au catcher foils actually used in the ${ }^{12} \mathrm{C}-248 \mathrm{Cm}$ system. Hoivever, some tailing of the ${ }^{246} \mathrm{Cf}$ and ${ }^{252} \mathrm{Fm}$ occurred up to $0.7 \mathrm{mg} / \mathrm{cm}^{2}$ in $\mathrm{Al}$, corresponding to about 2 $\mathrm{mg} / \mathrm{cm}^{2}$ in $\mathrm{Au}$. Once again though, the Au catcher foils should be sufficiently thick to stop these recoiling target-like fragments. From the range of $252 \mathrm{Fm}$ nuclei in the $\mathrm{Al}$, the average recoil energy is about $5 \mathrm{MeV}$, and the maximum recoil energy is approximately $15 \mathrm{MeV}$. As Moody [MOO83] points out, there is measurable activity in the first foils from the stack experiments of McFarland [MCF82]; hence, there is a probability that a few percent of the recoiling reactions products were stopped in the target material. In fact, Moody attributes discrepancies between Bk cross sections reported for two identical 
experiments he performed (except for target thickness) as "probably partly due recoil losses in the thicker target" [MOO83].

Considering the experiments where ${ }^{248} \mathrm{Cm}$ was bombarded with $93 \mathrm{MeV} \quad{ }^{12} \mathrm{C}$ ions, if compound nucleus formation takes place, then the recoiling 260 No compound nucleus products should have about $4.3 \mathrm{MeV}$ of kinetic energy assuming no kinetic energy is converted to excitation energy. The range of $4.3 \mathrm{MeV}{ }^{260}$ No ions in the target material is about $0.6 \mathrm{mg} / \mathrm{cm}^{2}$ [NOR70], much greater than the $0.182 \mathrm{mg} / \mathrm{cm}^{2}$ thickness of the ${ }^{248} \mathrm{Cm}$ target. Likewise, the range of $4.3 \mathrm{MeV} 260$ No ions in $\mathrm{Au}$ is about $0.5 \mathrm{mg} / \mathrm{cm}^{2}$; hence, the $2.47 \mathrm{mg} / \mathrm{cm}^{2}$ Au catcher foils used in this set of experiments are more than adequate to stop the recoiling compound nucleus products.

At the other extreme, consider the case of compound nucleus formation from $71 \mathrm{MeV}$ ${ }^{12} \mathrm{C}$ ions interacting with ${ }^{248} \mathrm{Cm}$. The recoiling ${ }^{260} \mathrm{No}$ ions should have about $3.3 \mathrm{MeV}$ of kinetic energy, again assuming no excitation energy. The range of $3.3 \mathrm{MeV}{ }^{260} \mathrm{No}$ ions in the target is about $0.4 \mathrm{mg} / \mathrm{cm}^{2}$, once again greater than the $0.182 \mathrm{mg} / \mathrm{cm}^{2}$ target thickness. The range of $3.3 \mathrm{MeV}{ }^{260} \mathrm{No}$ ions in the Au catcher foil material is approximately $0.4 \mathrm{mg} / \mathrm{cm}^{2}$, compared to the $2.47 \mathrm{mg} / \mathrm{cm}^{2}$ thickness of the Au catcher foil. Similar arguments can be made for the ${ }^{31} \mathrm{P},{ }^{40} \mathrm{Ar}$, and ${ }^{44} \mathrm{Ca}$ systems.

Türler considered the scenario at higher bombarding energies where the target-like reaction products are emitted at angles near the limits of the target arrangement [TÜR89]. The center-of-mass scattering angle $\theta_{c m}$ is smaller than the calculated grazing angle for the projectile-like fragment if the projectile and target stick together and rotate for a finite length of time. Similarly for the target-like reaction products, recoils at angles larger than the grazing angle may be possible. Considering the ${ }^{44} \mathrm{Ca}-{ }^{248} \mathrm{Cm}$ system at projectile energies of 230.8-236.5 MeV, Türler found losses of projectile-like products due to absorption in the target material at scattering angles of $180^{\circ}-\theta_{\mathrm{cm}} \geq 140^{\circ}$ (This corresponds to a scattering angle in the laboratory frame of reference of $\Omega_{l a b}=$ $\left.68^{\circ}\right)$ 
From the above arguments, it can be seen that even for the most extreme case studied here $\left({ }^{31} \mathrm{P}\right.$ at $\left.7 \mathrm{MeV} / \mathrm{u}\right)$, the probability of a recoiling target-like product hitting the catcher foil is 0.95 . Therefore these losses can be ignored especially at the lower bombarding energies. Additionally, it has been shown that the catcher foils were sufficiently thick to catch those products with the maximum possible recoil energy. It will be assumed in all of the following calculations that $100 \%$ of the recoiling reaction products are caught by the catcher foils.

\section{Experimental Conditions}

The Experimental conditions for each irradiation are shown in Table II.3. All of the beam energies have been corrected for the appropriate energy loss in the target system and represent the average energy of the ions in the ${ }^{248} \mathrm{Cm}$ target material in the laboratory frame of reference. The energy loss of the beam in the target was calculated using range and stopping power tables for heavy ions [HUB80] [NOR70]. Several values had to be estimated due to insufficient data in the range and stopping power tables. The stopping powers of oxygen and nitrogen were interpolated from the stopping powers of adjacent elements, while uranium was used as an approximation for curium. The stopping power for the complex alloy HAVAR was approximated with $\mathrm{Ni}$. The composition of HAVAR is shown in Table II.4 [GRE85].

\section{E. Manual Chemical Separations}

Heavy actinide transfer reaction products constitute only a small fraction of the nuclides produced from the interactions of heavy ions with actinide targets. Typical actinide production cross sections are on the order of microbarns whereas the total reaction cross section is approximately one barn. Therefore, chemical separation procedures must be employed in order to isolate the actinide products of interest. The chemical separations, however, must meet several criteria. First of all they must effectively and cleanly separate the actinides from other interfering activities such as the lanthanides. Secondly, the actinides must be separated from each other. Separate 
actinide fractions allow $Z$ identification and aid in the determination of independent production yields of the actinides of interest. Separate elemental fractions are also vital when two nuclides with similar $t / 1 / 2$ 's emit gamma rays with the same energy (i.e. ${ }^{250} \mathrm{Es}^{\mathrm{m}}$ $t_{1 / 2}=2.2 \mathrm{~h}$ and $250 \mathrm{Bk} t_{1 / 2}=3.22 \mathrm{~h}$ ) [KEG85]. In addition, the chemical separation procedures should be efficient and relatively fast in order to isolate nuclides with half lives on the order of 30 to 45 minutes.

Ion exchange chromatography has become one of the most important tools for the separation of the lanthanides and actinides [STE61]. The resins used in ion exchange chromatography are simply three-dimensional structures with numerous ionizable groups affixed. The most common resins are polystyrenes cross-linked with divinylbenzene [DYE61]. The degree of cross-linking is directly proportional to the porosity of the resin. High porosity is desired since this feature will determine the number of available sites for ion exchange to take place and will therefore increase the selectivity of the resin [STE61]. In addition, greater cross-linking decreases the elasticity of the three dimensional matrix and hence the swelling that takes place is reduced [MAS71].

\section{Anion Exchange Chromatography}

Anion exchange resins exchange anionic species between the stationary resin phase and the mobile liquid phase. The active sites in the resin are typically quaternary amines. The elution position, or chromatographic retention of an element is dependent upon the competition for the element between the stationary resin phase and the complex in the mobile liquid phase. Ions with different charge densities form complexes of different stabilities. Hence the elution positions of different species vary and an effective separation can be performed [COO52].

\section{Cation Exchange Chromatography}

Cation exchange resins exchange cationic species between the resin phase and the mobile liquid phase according to

$$
A^{+}+R^{-} B^{+} \rightleftharpoons B^{+}+R^{-} A^{+}
$$


where $A^{+}$is the cationic species of interest, $B^{+}$is mobile phase ion and, $R^{-}$is the ionic exchange site [BAU78]. Typical cation exchange resins consist of cross-linked polystyrene with sulfonic acid groups $\left(-\mathrm{SO}_{3} \mathrm{H}\right)[\mathrm{FRI} 81]$. Separations usually involve the adsorption of ions from a solution on the resin followed by the selective desorption of the elements of interest from the column with different solutions [STE61].

\section{$\alpha$-Hydroxyisobutyrate Separations}

The elements Am through Lr, like the analogous lanthanides (Eu through Lu), all exist as +3 ions in aqueous solutions except for No which exists as a +2 ion. This similarity presents a problem in the separation of the heavier actinides. They can, however, be separated on the basis of their trivalent ionic radii. It is well known that the ionic radii of the actinides decrease in going from $\mathrm{Ac}^{+3}(0.118 \mathrm{~nm})$ to $\mathrm{Lr}^{+3}(0.088 \mathrm{~nm})$ The ionic radii of the +3 actinides are listed in Table II.5 [BRA86].

A very common method for the separation of the lanthanides and transcurium elements from each other, based upon ionic radii, is the elution of the trivalent ions from a cation exchange resin with a complexing agent. Most commonly, organic acid anions such as citrate, glycolate, lactate, and $\alpha$-hydroxyisobutyrate $(\alpha-H I B)$ are used [HIG60)]. However, ammonium $\alpha$-hydroxyisobutyrate typically gives better separation factors and has become one of the most widely used complexing agents [CHO56] [MAS71] |SMI56|.

It has been shown that when $\alpha$-HIB is present in concentrations of $0.1 \mathrm{M}$ or greater, it will form - 1 charged species with +3 ions of the form,

$$
4 L^{-}+M^{+3} \rightleftharpoons\left|M L_{4}\right|^{-}
$$

where $L$ represents $\alpha$-hydroxyisobutyrate and $M$ is the metal ion of interest [CHO61]. Complexing agents such as $\alpha$-hydroxyisobutyrate stabilize the cation in the mobile phase. Cations with higher charge densities will therefore spend a greater amount of time in the mobile phase when compared to cations with smaller charge densities. Hence, the cations with the highest charge densities will be eluted first. Since the charge 
density of the actinides increases as the atomic number increases, the actinide elements are eluted from the cation exchange resin in order of decreasing atomic number.

\section{Reverse Phase HDEHP Column Chromatography}

\section{a. Separation of the Actinides from the Lanthanides}

The lanthanides, produced primarily by fission, are major products of heavy ion reactions with actinide targets. Because they are produced in large quantities, if they are present in the actinide fractions, their activities would mask the actinide activities. In order to collect radiochemically pure samples so that clean gamma spectra could be obtained, it was necessary to separate the actinides from the lanthanicies. The separation of actinides from lanthanides is not trivial due to the similarities in chemical properties and ionic radii. The actinides, however, form stronger chloride and thiocyanate complexes than the corresponding lanthanides [HIG60)]. Based on this property, an effective separation of actinides from lanthanides can be achieved on a cation exchange resin column using $13 \mathrm{M} \mathrm{HCl}$ as the eluent. Since the actinides form stronger neutral or anionic chloride complexes, they are more stable in the mobile phase and elute from the column more quickly than the lanthanides.

\section{b. Elution of Actinide Fractions Containing Short-Lived Nuclides Via Reverse}

\section{Phase HDEHP Column Chromatography}

The $\alpha$-HIB chemical separation procedure can be too time consuming for the quick elution of elemental fractions containing short-lived nuclides such as ${ }^{251} \mathrm{Bk}\left(t_{1} / 2=57 \mathrm{~m}\right)$. If one waits for the isolation of the Bk fraction on the $\alpha$-hydroxyisobutyrate column, which is generally the last step in the chemical separation scheme, 3 or 4 hours could elapse, resulting in very little remaining $251 \mathrm{Bk}$ activity for gamma-ray counting. An alternative separation technique, utilizing reverse phase column chromatography can be used to produce a chemical fraction containing Bk quickly. Reverse phase column chromatography utilizes a polar mobile phase and a nonpolar stationary phase such as silica particles with hydrocarbon chains attached to the surface [DOR89]. Hence, the 
transplutonium elements can be effectively and quickly separated by a column of bis(2ethylhexyl) orthophosphoric acid (HDEHP) adsorbed on hydrophobic diatomaceous earth (Celite 545) as the stationary phase with thineral acids as the mobile phase [HOR69] [HOR69A] [HOR72] [WIN63]. With this technique, the transplutonium elements are eluted in order of increasing $\mathrm{Z}$, resulting in a separation of the Bk fraction in about one hour.

\section{Recoil Atom Collection Foils}

\section{a. Chemical Procedure for Copper Catcher Foils}

After each irradiation, the copper catcher foil was removed and taken to a chemistry laboratory. The foil was dissolved in $1 \mathrm{ml}$ of a solution of $15.8 \mathrm{M} \mathrm{HNO}_{3}$ with $50 \mu \mathrm{l}$ of $70 \% \mathrm{HClO}_{4}$. Appropriate yield tracers such as ${ }^{249} \mathrm{Cf}$ were added in order to monitor the chemical yield of the actinides throughout the separation procedure. After dissolution, the solution was evaporated to dryness until $\mathrm{NO}_{2}$ fumes were evolved. The resulting residue was dissolved in $1 \mathrm{ml}$ of $10 \mathrm{M} \mathrm{HNO}_{3}$ and cooled to room temperature. When the solution reached room temperature it was sorbed onto a $3-\mathrm{mm}$ diameter $\mathrm{X}$ 60-mm long column of Bio Rad AG-1, X-8 (200-400 mesh) anion exchange resin which had been pretreated with $10 \mathrm{M} \mathrm{HNO}_{3}$. The trivalent actinides, $\mathrm{Ra}$, and $\mathrm{Cu}$ were eluted with approximately $0.5 \mathrm{ml}$ of $10 \mathrm{MHNO}_{3}$. Th, $\mathrm{Pa}, \mathrm{U}, \mathrm{Np}$, and $\mathrm{Pu}$ remained on the column and were subsequently eluted with various solutions. These activities, however, were not used in this study and will not be discussed in this text. A full treatment of the Th.Pu activities produced in the interactions of ${ }^{44} \mathrm{Ca}$ with ${ }^{248} \mathrm{Cm}$ is given in [TÜR89]. The effluent containing the trivalent actinides, $\mathrm{Ra}$, and $\mathrm{Cu}$ was evaporated to dryness. The remaining activity was dissolved in about $1 \mathrm{ml}$ of $9 \mathrm{M} \mathrm{HCl}$ and passed through a $10-\mathrm{mm}$ diameter X 50 mm long column of Bio Rad AG-1, X-8 (200)-400 mesh) anion exchange resin which had been pretreated with $9 \mathrm{M} \mathrm{HCl}$. The trivalent actinides and Ra were eluted from the column with the $9 \mathrm{M} \mathrm{HCl}$ while $\mathrm{Cu}$ was adsorbed on the resin.

The effluent from the second anion exchange column was evaporated to dryness and 
the remaining activity was dissolved in $0.1 \mathrm{M} \mathrm{HCl}$ and passed through a $2-\mathrm{mm}$ diameter X 50-mm long column of Bio Rad AG, MP-50 (200-400) mesh) cation exchange resin. This column had previously been treated with $0.1 \mathrm{M} \mathrm{HCl}$. Ac and Am through $\mathrm{Md}$ were eluted with freshly saturated $13 \mathrm{M} \mathrm{HCl}$ in the first four free column volumes, ahead of $\mathrm{Ra}$ and the lanthanides. A known amount of ${ }^{170} \mathrm{Tm}$ tracer was added to the resulting fraction containing Ac and Am through Md. This fraction was dried, dissolved in $0.1 \mathrm{ml}$ $\mathrm{H}_{2} \mathrm{O}$ and dried again to ensure the removal of any residual acid. The residue was dissolved in about $0.2 \mathrm{ml}$ of $0.02 \mathrm{M} \mathrm{HNO}_{3}$ and passed through a 2-mm diameter X 50mm long column of HDEHP on hydrophobic Celite 535. Actinium was eluted in $\mathrm{t}$.. third free column volume with $0.1 \mathrm{M} \mathrm{HNO}_{3}$. Upon adding $0.04 \mathrm{MHNO}_{3}, \mathrm{Am}$ and $\mathrm{Cm}$ were eluted in the second free column volume with Bk coming off in the fourth free column volume. $0.08 \mathrm{M} \mathrm{HCl}$ was then used to elute $\mathrm{Cf}$ through $\mathrm{Md}$ from the column.

To both the Am and $\mathrm{Bk}$ fractions, $1 \mathrm{mg}$ each of La carrier and $\mathrm{Zr} / \mathrm{Ba}$ hold-back carriers were added. $1 \mathrm{ml}$ of $1 \mathrm{M}$ HF was then added resulting in the coprecipitation of $\mathrm{AmF}_{3}$, and $\mathrm{BkF}_{3}$ along with the $\mathrm{LaF}_{3}$ in each fraction. Millipore type GS $(22 \mu \mathrm{m})$ nitrocellulose filters were used to filter the precipitates. The dried precipitates were then mounted for gamma-ray spectroscopy.

The fraction containing the Cf, Es, Fm, and Md was heated to dryness to remove any residual acid and subsequently dissolved in $0.5 \mathrm{M} \mathrm{HCl}$ and sorbed on a $2-\mathrm{mm}$ diameter by $50-\mathrm{mm}$ long column of Dowex-50, X-12 cation exchange resin $(7-10 \mu \mathrm{m})$ kept at 80 ${ }^{\circ} \mathrm{C}$. The $\mathrm{HCl}$ was removed from the column with 10()$\mu \mathrm{l}$ of $\mathrm{H}_{2} \mathrm{O}$. The trivalent actinides were eluted from the column in separate fractions using $0.5 \mathrm{M}$ ammonium alphahydroxyisobutyrate $(\alpha$-HIB) at a $\mathrm{pH}$ of 3.80 . The purified chemical fractions were collected on Pt discs in 2-drop increments. Each disc was monitored for alpha and spontaneous fission (SF) activities with a windowless $2 \pi$ gas flow detector and for $B^{-}$ activity with a proportional counter. Those discs containing the same chemical element were combined on a single piece of $\mathrm{Pt}$, ctried, flamed, and mounted on aluminum 
counting cards. The chemical procedure used on the copper catcher foils is represented schematically in Fig. II.4 and Fig. II.5.

\section{b. Chemical Procedure for Gold Catcher Foils}

After the first three experiments, it was realized that the interferences from other activities were small, therefore the chemical separations could be greatly simplified while still allowing accurate detection of the decay of the actinide nuclides of interest. Specifically, the cation exchange column utilizing AG MP-50 and the HDEHP reverse phase chromatographic column were excluded. Gold recoil catcher foils were used rather than copper, further simplifying the procedure. These modifications reduced the chemical separation times from 3.5 hours to about 2 hours. In addition, before each long bombardment a short bombardment of approximately 1 hour was performed. The short bombardments allowed the normalization of the +3 actinide chemical yields of the long bombardments and the determination of short-lived ${ }^{250} \mathrm{Fm}\left(t_{1 / 2}=30 \mathrm{~m}\right)$ cross sections.

The recoil products from the short bombardments were caught on a gold catcher foil which was subsequently removed and dissolved in approximately $0.25 \mathrm{ml}$ of hot aqua regia containing a known amount of ${ }^{241} \mathrm{Am}$ tracer. An ether extraction was performed on this solution to remove about $95 \%$ of the gold [MYL11]. The aqueous solution was heated in a water bath to remove the dissolved ether and subsequently passed through a 3-mm diameter X 10-mm long column of Bio Rad AG-1, X-8 anion exchange resin in 12 $\mathrm{M} \mathrm{HCl}$. The remaining gold was adsorbed on the column while the trivalent actinides passed through the column. The effluent was heated to dryness, redissolved in $3 \mathrm{M} \mathrm{HCl}$ and aken to dryness again to remove any residual nitric acid. The remaining activity was dissolved in $3 \mathrm{M} \mathrm{HCl}$ and dried on a small piece of platinum, which was then mounted on an aluminum counting card for alpha-particle pulse height analysis. A flowchart of the chemical procedure for the gold foils from the short irradiations is given in Fig. II.6.

The gold catcher foil from each long bombardment was removed and dissolv $\mathrm{d}$ in hot 
aqua regia containing a known amount of ${ }^{241} \mathrm{Am}$ tracer. An ether extraction was performed, the aqueous solution was heated in a water bath to remove the dissolved ether, and then passed through a 3-mm diameter X 50-mm long column of Bio Rad AG$1, \mathrm{X}-8$ anion exchange resin in $12 \mathrm{M} \mathrm{HCl}$. The eluate was evaporated to dryness and fumed twice with concentrated $\mathrm{HClO}_{+}$to remove any residual organic material. The remaining activity was dissoived in $0.5 \mathrm{M} \mathrm{HCl}$ and sorbed on a 2-mm diameter by 50 $\mathrm{mm}$ long column of cation exchange resin (Dowex- 50, X-12, 7-10 $\mu \mathrm{m}$ ) maintained at 80 ${ }^{\circ} \mathrm{C}$. The column was washed with approximately $100 \mu \mathrm{l}$ of $\mathrm{H}_{2} \mathrm{O}$ to remove the $\mathrm{HCl}$. Separate fractions of the trivalent actinides were eluted with $0.5 \mathrm{M}$ ammonium alphahydroxyisobutyrate of $\mathrm{pH} 3.80$. The eluate was collected on $\mathrm{Pt}$ discs in two-drop increments and dried. The discs were monitored for alpha and spontaneous fission activities with a windowless $2 \pi$ gas flow detector, and for $B^{-}$activity with a proportional counter. The activity on those discs containing the same chemical element were combined on a single piece of $\mathrm{Pt}$ for pulse height analysis. The chemical procedure used on the Au catcher foils from the long bombardments is presented in Fig. II.7.

\section{F. Automated Chromatographic Chemical Element Separator System}

It was desired to measure the cross sections of the various $\mathrm{Md}$ isotopes produced in the interactions of ${ }^{12} \mathrm{C}$ with ${ }^{248} \mathrm{Cm}$. Due to the low production cross sections and short half-lives, a procedure was needed which incorporated reproducible chemical separations of the $\mathrm{Md}$ from the other reaction products on a time scale comparable to the half-lives of the Md isotopes of interest $\left(254 \mathrm{Md} t_{1 / 2}=10 \mathrm{~m}, 255 \mathrm{Md} t_{1 / 2}=27 \mathrm{~m}\right.$, and $\left.256 \mathrm{Md} t_{1 / 2}=76 \mathrm{~m}\right)$. The Automated Chromatographic Chemical Element Separator System (ACCESS) was designed exactly for this type of separation [PAL88]. ACCESS is a computer-controlled separation system composed of high-performance liquid chromatography (HPLC) pumps ard pneumatically actuated valves which guide a variety of solutions through a sample injection loop, and a chromatography column. The computer also controls an autornated fraction collector. The modular design of ACCESS allows for great flexibility in the 
type of separations that can be performed. For example, anion exchange, cation exchange, and reverse-phase extraction chromatography can be used with ACCESS to perform rapid, chemical separations. Two schematic diagrams of ACCESS are illustrated iı Figs. II.8 and II.9.

Instead of using $\mathrm{Au}$ or $\mathrm{Cu}$ catcher foils to stop and collect the reaction products, a $\mathrm{He} / \mathrm{KCl}$-jet system was employed. The recoiling reaction products were thermalized in He (1.2 bar) which was seeded with $\mathrm{KCl}$ aerosol. After the thermalized reaction products became attached to the aerosol, the $\mathrm{KCl}$ aerosol was pumped through a polyvinyl chloride capillary tube to a collection site located above the cave shielding. The activityladen $\mathrm{KCl}$ aerosol was collected for approximately 20 minutes on $2 \mathrm{~cm} \times 2 \mathrm{~cm}$ pieces of Teflon. At the end of the collection period, the $\mathrm{KCl}$ was dissolved in $95 \mu \mathrm{l}$ of $0.05 \mathrm{M}$ ammonium alpha-hydroxyisobutyrate ( $\mathrm{pH} \cong 2$, not adjusted), and introduced into ACCESS via the sample injection loop. This solution was sorbed on a $2-\mathrm{mm}$ diameter $\mathrm{X}$ 70-mm long column containing cation exchange resin (Hamilton $\mathrm{X}-12,10-15 \mu \mathrm{m}$ ) kept at $80^{\circ} \mathrm{C}$. The trivalent actinides were eluted in order of decreasing $\mathrm{Z}$ with $0.5 \mathrm{M}$ ammonium alpha-hydroxyisobutyrate $(\mathrm{pH}=3.36)$. The isolated $\mathrm{Md}$ and Fm fractions were collected in polyethylene centrifuges cones via an automated fraction collector, evaporated to dryness on Pt discs, and flamed for alpha pulse height analysis. Table II.6 lists the specific collection times and beam integrals for the experiments performed with ACCESS. It is estimated that the efficiency of the $\mathrm{He} / \mathrm{KCl}$-jet transport system is $75 \%$. This efficiency was determined by comparing the yields of Fm isotopes from the transport system to those from a separate 1-h irradiation utilizing a Au catcher foil to collect the recoiling product atoms. The chemical procedure used on the Au foils from these 1-h irradiations is identical to that described in II.E.4.b.

\section{G. Data Acquisition}

\section{Merry-Go-Round Realtime Data Acquisition and Graphics System}

A rotating wheel system was used for the on-line measurements of the No cross 
sections from the $\left({ }^{12} \mathrm{C}, x n\right)$ reactions with ${ }^{248} \mathrm{Cm}$. The $\mathrm{He} / \mathrm{KCl}$-jet system was again utilized to transport the recoiling reaction products from the target system to the "MerryGo-Round" (MG) horizontal, rotating wheel system (see Fig II.10) [HOF80]. The MG wheels rotated through a series of six passivated, ion-implanted, planar silicon detector pairs (100 $\mathrm{mm}^{2}$ active area, 300 -micron depletion depth) mounted above and below the plane of the wheel (see Fig. II.11). Thin polypropylene foils $\left(40 \pm 15 \mu \mathrm{g} / \mathrm{cm}^{2}\right)$, mounted on $12.67 \mathrm{~mm}$ steel washers $(6.3 \mathrm{~mm}$ i.d. hole), were placed at 80 equally spaced positions on the periphery of each MG wheel.

The activity-laden $\mathrm{KCl}$ aerosol was deposited on the first polypropylene foil for a preset time, after which the wheel was rotated $4.5^{\circ}$, moving the first collection foil from the collection site to a position located between the first pair of silicon detectors. After the second collection time, the wheel was rotated again, moving the first collection foil between the second pair silicon detectors, and moving the second collection foil between the first pair of detectors. This process continued until all 80 collection positions had been used. The MG wheel was replaced with a new one after about five complete revolutions in order to minimize the accumulation of any long-lived activities.

The $\alpha$ particle detection efficiency was determined to be $30 \%$. The efficiency of the $\mathrm{He} / \mathrm{KCl}$-jet was determined with a separate $1-\mathrm{h}$ irradiation utilizing an $\mathrm{Au}$ catcher foil as described earlier. The output signals from the ion implanted silicon detectors were digitized by Ortec AD-811 ADC's which were controlled by a Standard Engineering CAMAC crate controller. All events were stored on magnetic tape, in list mode, by our Realtime Data Acquisition and Graphics System (RAGS). Each event was tagged with a time, channel number (proportional to alpha particle energy), and detector number.

\section{Counting Procedure}

High purity $\mathrm{Ge}$ or $\mathrm{Ge}(\mathrm{Li})$ gamma-ray spectrometer systems were used to assay the $\mathrm{Bk}$ fractions. The Fm fractions were assayed with a fission-alpha spectrometer system utilizing $\mathrm{Si}(\mathrm{Au})$ surface barrier detectors with active areas of $100 \mathrm{~mm}^{2}$. In the ${ }^{44} \mathrm{Ca}$ 
experiments, the $\mathrm{Cf}$ fractions were first assayed on a gamma-ray spectrometer system to observe the $\mathrm{K} x$-rays from the electron capture decay of ${ }^{247} \mathrm{Cf}$, and then on a fissionalpha spectrometer system. However, for the ${ }^{40} \mathrm{Ar},{ }^{31} \mathrm{P}$, and ${ }^{12} \mathrm{C}$ experiments, the $\mathrm{Cf}$ fractions were assayed only on the fission-alpha spectrometer system. The Es samples were simultaneously assayed for alpha, gamma, and spontaneous fission activities by mounting the purified samples in a vacuum chamber containing a $100 \mathrm{~mm}^{2} \mathrm{Si}(\mathrm{Au})$ surface barrier detector. This chamber was then positioned next to a $\mathrm{Ge}(\mathrm{Li})$ gamma-ray detector. A schematic diagram of the alpha-gamma counting chamber is displayed in Fig. II.12. All of the separated samples were continuously counted for four weeks after the end of bombardment and at appropriate intervals so that unambiguous nuclide identification could be established by measuring both the decay energies and the halflives.

\section{H. Data Processing and Calculations}

\section{Processing of Alpha Data}

Each alpha-spectrum consisted of a 1024-channel histogram with all fission events (events above a $30 \mathrm{MeV}$ threshold) stored in channel zero. A typical Cf fraction alphaspectrum is shown in Fig. II.13. All of the alpha spectra from each elemental fraction were summed so that every peak present could be located and tentatively identified based upon characteristic alpha-decay energies. This summing procedure aided in the identification of low intensity peaks which might not have been easily seen in any individual spectrum. Once all of the peaks were identified, regions of interest were set around the appropriate peaks. A region of interest was also set on channel zero to include any fissioning activities that might be present. The regions of interest in the individual spectra were integrated using INTEG2, a simple computer code [HAL86]. INTEG2 summed the counts in each channel within the regions of interest and constructed a decay curve for each region based on these summed counts and the times of measurement for each spectrum. The decay curves were subsequently fit by an error 
weighted non-linear least squares program, EXFIT [GRE85]. Activities at the end of bombardment and the associated errors were calculated from the decay curve fitting procedure of EXFIT. Once the initial activities were calculated, the absolute count rates were determined by correcting for the abundarices of specific decay modes, detector efficiencies, chemical yields, and the fraction of the alpha peaks contained within the regions of interest. Table II.7 lists the $\alpha$ emitters used in this study along with the associated half-lives, decay energies, and $\alpha$ decay branches. The nuclides measured which decay by spontaneous fission are shown in Table II.8.

In order to determine the efficiencies of each $100 \mathrm{~mm}^{2} \mathrm{Si}(\mathrm{Au})$ surface barrier detector, a standard alpha source containing a known amount of $241 \mathrm{Am}$ was prepared before each experiment. The efficiencies typically ranged from 16 to $21 \%$ with the efficiency for fission fragments assumed to be twice that of alpha particles.

\section{Processing of Gamma Data}

Each gamma spectrum consisted of a 4096-channel histogram. Fig. II.14 shows the first 2000 channels of a representative gamma-spectrum from a $\mathrm{Bk}$ fraction eluted from the $\alpha$-HIB column. The gamma spectra from each elemental fraction were analyzed using the SAMPO computer code [ROU69]. This code contains algorithms for line-shape, energy, and efficiency calibrations. In addition it has automatic peak-search and peakfitting routines, as well as the ability to deconvolute multiplet peaks. Decay curves were constructed from the peak areas found by SAMPO. Initial activities were calculated with CLSQ [CUM63], a non-linear, least squares, multi-component decay curve fitting program. Abundances of the specific gamma-decay energies were used to calculate the absolute disintegration rates at the end of bombardment of the nuclides of interest. Table II.9 lists some decay information for the nuclides of interest which decay by electron capture (EC) or beta emission ( $\left(^{-}\right)$.

\section{Calculation of Cross Sections}

The number of product nuclei produced per unit time, $d N_{p} / d t$, for a constant beam of 
particles striking a thin target is

$$
\frac{d N_{p}}{d t}=I N_{t} \sigma
$$

where $l$ is the number of incident particles striking the target per unit time, $N_{t}$ is the number of target atoms per $\mathrm{cm}^{2}$, and $\sigma$ is the cross section for the production of this product. However, if the nuclide being produced is unstable and decays during the bombardment, then the net rate of production of this nuclide is

$$
\frac{d N_{p}}{d t}=I N_{t} \sigma-\lambda_{p} N_{p}
$$

where $\lambda_{p}$ is the decay constant of the product and $N_{p}$ is the number of product nuclei present at time $t$. Assuming that $I$ is constant, Eq. 2 can be solved by rearrangement, introduction of an integrating factor, and finally integration to give [STE66]

$$
N_{p}=N_{p o} \exp \left[-\lambda_{p}\left(t-t_{o}\right)\right\}+\frac{N_{t} l \sigma\left\{1-\exp \left[-\lambda_{p}\left(t-t_{o}\right)\right]\right\}}{\lambda_{p}},
$$

where $N_{p}$ is the number of target nuclei present at the end of bombardment, $t$ is the end of bombardment time, $t_{O}$ is the start of bombardment, and $N_{p o}$ is the number of product nuclei present at the beginning of bombardment. If at the start of bombardment there are no product nuclei present, then Eq. 3 reduces to

$$
N_{p}=\frac{N_{t} I \sigma\left\{1-\exp \left[-\lambda_{p}\left(t-t_{o}\right)\right]\right\}}{\lambda_{p}} .
$$


Rearranging Eq. 4 and solving for $\sigma$, one obtains

$$
\sigma=\frac{\lambda_{p} N_{p}}{N_{t} I\left\{1-\exp \left[-\lambda_{p}\left(t-t_{o}\right)\right]\right\}}=\frac{A_{p}}{N_{t} I\left\{1-\exp \left[-\lambda_{p}\left(t-t_{o}\right)\right]\right\}}
$$

where $A_{p}$ is the activity of the product at the end of bombardment. In reality, the beam intensity is not constant, so the beam history must be divided into $j$ intervals giving

$$
\sigma=\frac{A_{p}}{N_{t} \Sigma l_{j}\left[1-\exp -\lambda_{p}\left(t_{j+1}-t_{j}\right)\right] \exp \left[-\lambda_{p}\left(t-t_{j+1}\right)\right]},
$$

where $I_{j}$ is the average beam intensity during the $j$ th interval, $t_{j}$ is the time at the start of the $j$ th interval, and $t$ is the time at the end of bombardment. Therefore, Eq. (9) was used to calculate the cross sections of the nuclides of interest. 
Table II.1. Projectile, ${ }^{248} \mathrm{Cm}$ target thicknesses, catcher foil material, and catcher foil thicknesses.

\begin{tabular}{lllll}
\hline & & & & \\
\hline Exp \# & Projectile & $\begin{array}{c}\text { Target } \\
\text { Thickness } \\
\left(\mathrm{mg} / \mathrm{cm}^{2}\right)\end{array}$ & $\begin{array}{c}\text { Catcher Foil } \\
\text { Material }\end{array}$ & $\begin{array}{c}\text { Catcher Foil } \\
\text { Thickness } \\
\left(\mathrm{mg} / \mathrm{cm}^{2}\right)\end{array}$ \\
\hline & & & & \\
$1-3$ & ${ }^{44} \mathrm{Ca}$ & 0.644 & $\mathrm{Cu}$ & $5.7-7.0$ \\
4,5 & ${ }^{44} \mathrm{Ca}$ & 0.644 & $\mathrm{Au}$ & 19.6 \\
$6-8$ & ${ }^{40} \mathrm{Ar}$ & 0.644 & $\mathrm{Au}$ & 19.6 \\
9,10 & $40 \mathrm{Ar}$ & 0.491 & $\mathrm{Au}$ & 12.3 \\
$11-15$ & $31 \mathrm{P}$ & 0.491 & $\mathrm{Au}$ & 12.3 \\
$16-20$ & $12 \mathrm{C}$ & 0.182 & $\mathrm{Au}$ & 2.47 \\
\hline
\end{tabular}


Table II.2. Maximum recoil energy, range in catcher foil, thickness of catcher foil, and composition of catcher foil for experimental systems used in this study.

\begin{tabular}{lcccc}
\hline Proj & $\begin{array}{c}\text { Max. Recoil } \\
\text { Energy } \\
(\mathrm{MeV})\end{array}$ & $\begin{array}{c}\text { Range in } \\
\text { Catcher Foil } \\
\left(\mathrm{mg} / \mathrm{cm}^{2}\right)\end{array}$ & $\begin{array}{c}\text { Thickness of } \\
\text { Catcher Foil } \\
\left(\mathrm{mg} / \mathrm{cm}^{2}\right)\end{array}$ & $\begin{array}{c}\text { Catcher Foil } \\
\text { Material }\end{array}$ \\
\hline${ }^{12} \mathrm{C}$ & 15 & 2.1 & 2.47 & $\mathrm{Au}$ \\
${ }^{31} \mathrm{P}$ & 81 & 8.7 & 12.3 & $\mathrm{Au}$ \\
${ }^{40} \mathrm{Ar}$ & 124 & 11.2 & 12.3 & $\mathrm{Au}$ \\
${ }^{44} \mathrm{Ca}$ & 141 & 12.0 & 19.6 & $\mathrm{Au}$ \\
${ }^{44} \mathrm{Ca}$ & 141 & 5.7 & 7.0 & $\mathrm{Cu}$ \\
\hline
\end{tabular}


Table II.3. Experimental conditions for the long irradiations of ${ }^{248} \mathrm{Cm}$ with ${ }^{44} \mathrm{Ca}$, ${ }^{40} \mathrm{Ar},{ }^{31} \mathrm{P}$, and ${ }^{12} \mathrm{C}$ ions.

\begin{tabular}{|c|c|c|c|c|c|}
\hline Exp \# & Proj. & Charge State & $\begin{array}{c}\text { Ave. Energy in } \\
\text { the Target } \\
(\mathrm{MeV})\end{array}$ & $\begin{array}{c}\text { Length of } \\
\text { Bombardment } \\
\text { (min) }\end{array}$ & $\begin{array}{l}\text { Ave. } \\
\text { Beam } \\
\text { Flux } \\
(\operatorname{PnA})\end{array}$ \\
\hline 1 & ${ }^{44} \mathrm{Ca}$ & $10+$ & 231.7 & 650 & 90.0 \\
\hline 2 & ${ }^{44} \mathrm{Ca}$ & $10+$ & 251.9 & 474 & 28.0 \\
\hline 3 & ${ }^{44} \mathrm{Ca}$ & $12+$ & 274.8 & 763 & 18.3 \\
\hline 4 & ${ }^{44} \mathrm{Ca}$ & $12+$ & 297.0 & 326 & 11.7 \\
\hline 5 & ${ }^{44} \mathrm{Ca}$ & $12+$ & 319.1 & 450 & 16.7 \\
\hline 6 & ${ }^{40} \mathrm{Ar}$ & $9+$ & 207.0 & 412 & 77.8 \\
\hline 7 & ${ }^{40} \mathrm{Ar}$ & $11+$ & 224.9 & 276 & 59.1 \\
\hline 8 & ${ }^{40} \mathrm{Ar}$ & $11+$ & 245.0 & 480 & 31.8 \\
\hline 9 & ${ }^{40} \mathrm{Ar}$ & $11+$ & 265.5 & 641 & 36.4 \\
\hline 10 & ${ }^{40} \mathrm{Ar}$ & $11+$ & 285.5 & 415 & 29.1 \\
\hline 11 & $31 \mathrm{P}$ & $8+$ & 173.8 & 675 & 62.5 \\
\hline 12 & $31 \mathrm{P}$ & $8+$ & 190.0 & 654 & 100 \\
\hline 13 & ${ }^{31} \mathrm{P}$ & $8+$ & 207.4 & 743 & 93.8 \\
\hline 14 & $31 \mathrm{P}$ & $8+$ & 223.1 & 538 & 52.5 \\
\hline 15 & $31 \mathrm{P}$ & $8+$ & 238.9 & 647 & 50.0 \\
\hline 16 & ${ }^{12} \mathrm{C}$ & $4+$ & 70.7 & 355 & 675 \\
\hline 17 & ${ }^{12} \mathrm{C}$ & $4+$ & 76.4 & 595 & 800 \\
\hline 18 & ${ }^{12} \mathrm{C}$ & $4+$ & 83.6 & 484 & 800 \\
\hline 19 & $12 \mathrm{C}$ & $4+$ & 90.1 & 406 & 500 \\
\hline 20 & ${ }^{12} \mathrm{C}$ & $4+$ & 97.3 & 489 & 675 \\
\hline
\end{tabular}


Table II.4. Composition of the alloy HAVAR. Taken from [GRE85].

\begin{tabular}{cc}
\hline & \\
\hline Element & \% By Weight \\
\hline & \\
$\mathrm{Co}$ & 42.5 \\
$\mathrm{Cr}$ & 20.0 \\
$\mathrm{Fe}$ & 17.9 \\
$\mathrm{Ni}$ & 13.0 \\
$\mathrm{~W}$ & 2.8 \\
$\mathrm{Mo}$ & 2.0 \\
$\mathrm{Mn}$ & 1.6 \\
$\mathrm{C}$ & 0.2 \\
$\mathrm{Be}$ & 0.04 \\
\hline
\end{tabular}


Table II.5. Ionic radii of +3 actinides [BRA86].

\begin{tabular}{rcc}
\hline & & \\
\hline $\mathrm{Z}$ & Symbol & $\begin{array}{c}\text { Radius } \\
(\mathrm{nm})\end{array}$ \\
\hline 89 & $\mathrm{Ac}$ & 1.120 \\
90 & $\mathrm{Th}$ & 1.088 \\
91 & $\mathrm{~Pa}$ & 1.060 \\
92 & $\mathrm{U}$ & 1.035 \\
93 & $\mathrm{~Np}$ & 1.013 \\
94 & $\mathrm{Pu}$ & 0.994 \\
95 & $\mathrm{Am}$ & 0.976 \\
96 & $\mathrm{Cm}$ & 0.960 \\
97 & $\mathrm{Bk}$ & 0.945 \\
98 & $\mathrm{Cf}$ & 0.932 \\
99 & $\mathrm{Es}$ & 0.920 \\
100 & $\mathrm{Fm}$ & 0.909 \\
101 & $\mathrm{Md}$ & 0.898 \\
102 & $\mathrm{No}$ & 0.889 \\
103 & $\mathrm{Lr}$ & 0.880 \\
\hline & & \\
\hline
\end{tabular}


Table II.6 Experimental conditions for the irradiations of ${ }^{248} \mathrm{Cm}$ with $71-\mathrm{MeV}{ }^{12} \mathrm{C}^{+4}$ to produce Md isotopes.

\begin{tabular}{ccc}
\hline Run \# & $\begin{array}{c}\text { Length of } \\
\text { Irradiation } \\
(\mathrm{min})\end{array}$ & $\begin{array}{c}\text { Beam } \\
\text { Integral } \\
(\mu \mathrm{C})\end{array}$ \\
\hline & & \\
1 & 20 & 2740 \\
2 & 30 & 3991 \\
3 & 36 & 4623 \\
4 & 34 & 4136 \\
5 & 32 & 3723 \\
6 & 31 & 3917 \\
\hline
\end{tabular}


Table II.7. Decay properties of $\alpha$ emitters used in the present study [BRO78].

\begin{tabular}{|c|c|c|c|c|}
\hline \multicolumn{2}{|c|}{ Nuclide } & \multirow{2}{*}{$\frac{\text { Half Life }}{35.7 \mathrm{~h}}$} & \multirow{2}{*}{$\frac{\begin{array}{c}\alpha \text { Decay Energy* } \\
(\mathrm{MeV})\end{array}}{6.758}$} & \multirow{2}{*}{$\begin{array}{c}\text { Total } \alpha \\
\begin{array}{c}\text { Decay Branch } \\
(\%)\end{array} \\
100\end{array}$} \\
\hline $\mathrm{Cf}$ & 246 & & & \\
\hline & 248 & $333.5 \mathrm{~d}$ & 6.26 & 100 \\
\hline & 2.50 & $13.08 \mathrm{y}$ & 6.031 & 100 \\
\hline & 252 & $2.65 \mathrm{y}$ & 6.118 & 96.908 \\
\hline \multirow[t]{3}{*}{ Es } & 251 & $33.0 \mathrm{~h}$ & 6.492 & 0.49 \\
\hline & 252 & $471.7 \mathrm{~d}$ & 6.632 & 78 \\
\hline & 253 & $20.47 d$ & 6.633 & 100 \\
\hline \multirow[t]{5}{*}{$\mathrm{Fm}$} & 250 & $30.0 \mathrm{~m}$ & 7.44 & 100 \\
\hline & $25 ?$ & $25.39 \mathrm{~h}$ & 7.04 & 100 \\
\hline & 253 & $3.00 \mathrm{~d}$ & 6.943 & 12 \\
\hline & 254 & $3.24 \mathrm{~h}$ & 7.187 & 99.94 \\
\hline & 255 & $20.07 \mathrm{~h}$ & 7.022 & 100 \\
\hline
\end{tabular}

*Most abundiant decay eliergy. 
Table II.8. Decay properties of spontaneously fissioning nuclides [BRO78].

\begin{tabular}{rcc}
\hline & & \\
\hline Nuclide & $\begin{array}{c}\text { Total } \\
\text { Half Life }\end{array}$ & $\begin{array}{c}\text { SF } \\
\text { Branch }\end{array}$ \\
\hline Cf 252 & 2.64 y & 3.092 \\
254 & $60.5 \mathrm{~d}$ & 99.69 \\
Fm 256 & $2.627 \mathrm{~h}$ & 91.9 \\
\hline
\end{tabular}


Table II.9. Half lives, decay modes, photopeak energies, and absolute abundances of photopeaks for those nuclides decaying via electron capture (EC) or beta emission $\left(B^{-}\right)$ [BRO78].

\begin{tabular}{|c|c|c|c|c|c|}
\hline \multicolumn{2}{|c|}{ Nuclide } & \multirow{2}{*}{$\begin{array}{l}\text { Half Life } \\
4.35 \mathrm{~h}\end{array}$} & \multirow{2}{*}{$\frac{\text { Decay Mode }}{\text { EC }}$} & \multirow{2}{*}{$\begin{array}{c}\begin{array}{c}\text { Photopeak } \\
\text { Energy } \\
(\mathrm{keV})\end{array} \\
217.6\end{array}$} & \multirow{2}{*}{$\begin{array}{c}\begin{array}{c}\text { Absolute Abundanc } \\
\text { of Photopeak } \\
(\%)\end{array} \\
100^{*}\end{array}$} \\
\hline $\mathrm{Bk}$ & 244 & & & & \\
\hline & 245 & $4.90 \mathrm{~d}$ & EC & 252.9 & 31.3 \\
\hline & 246 & $1.80 \mathrm{~d}$ & EC & 798.7 & 61.4 \\
\hline & $248 m$ & $23.5 \mathrm{~h}$ & $B^{-}$ & 550.7 & 4.59 \\
\hline & 250 & $3.22 \mathrm{~h}$ & $\mathrm{~B}^{-}$ & 989.0 & 45.23 \\
\hline & 251 & $55.6 \mathrm{~m}$ & $B^{-}$ & 152.8 & $100^{*}$ \\
\hline $\mathrm{Cf}$ & 247 & $3.15 \mathrm{~h}$ & EC & $112.1^{+}$ & 33.7 \\
\hline Es & 249 & $1.70 \mathrm{~h}$ & $\mathrm{EC}$ & 379.5 & 40.4 \\
\hline & $250 m$ & $2.1 \mathrm{~h}$ & EC & 989.0 & 16.8 \\
\hline & $250 \mathrm{~g}$ & $8.6 \mathrm{~h}$ & $\mathrm{EC}$ & 829.0 & 73.6 \\
\hline & 251 & $33.0 \mathrm{~h}$ & $\mathrm{EC}$ & 177.6 & $\cong 2.0$ \\
\hline
\end{tabular}

${ }^{*}$ The absolute abundances are unknown and are therefore assumed to be $100 \%$.

${ }^{+}$This is actually the photopeak energy of the $K_{\alpha 1} \mathrm{x}$-ray from the Bk daughter. 


\section{Figure Captions}

Figure II.1. Target system schematic diagram. The distance between the graphite collimator and the HAVAR foil is about $40 \mathrm{~mm}$, the target is $3 \mathrm{~mm}$ from the HAVAR foil, the catcher foil is approximately $4 \mathrm{~mm}$ from the target, and the beam stop is about $120 \mathrm{~mm}$ from the catcher foil.

Figure II.2. Illustrative diagram of the calculations used to determine the probability of a recoiling reaction product missing the $\mathrm{Cu}$ and $\mathrm{Au}$ catcher foils as a function of laboratory angle. Taken from [MOO83].

Figure II.3. Recoil collection efficiency as a function of laboratory angle for the present target system in Cave 0 at the LBL 88-Inch Cyclotron.

Figure II.4. Flowchart of the chemical procedure used on the $\mathrm{Cu}$ catcher foils (Part I).

Figure II.5. Flowchart of the chemical procedure used on the $\mathrm{Cu}$ catcher foils (Part II).

Figure II.6. Flowchart of the chemistry used on the Au foils from the short irradiations.

Figure II.7. Chemistry flowchart for the Au foils from the long irradiations.

Figure II.8. Automated control system diagram for ACCESS. The Gilson FC 203 fraction collector is controlled by the personal computer (PC) through an RS-232C serial interface. The conditioning of the analytical column is controlled by software throughout each experiment. Schematic taken from [PAL88].

Figure II.9. Schematic diagram of ACCESS for Md experiments. Taken from [KAD90].

Figure II.10. Schematic diagram of the Merry-Go-Round Realtime Data Acquisition and Graphics System "MG-RAGS" at the LBL 88-Inch Cyclotron.

Figure II.11. Schematic diagram of "MG" wheel and detector arrangement.

Figure II.12. Alpha-gamma counting chamber used to assay isolated Es samples.

Figure II.13. Typical alpha spectrum from an isolated $\mathrm{Cf}$ fraction. 
Figure II.14. Typical gamma spectrum from an isolated Bk fraction. 


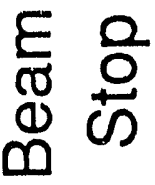
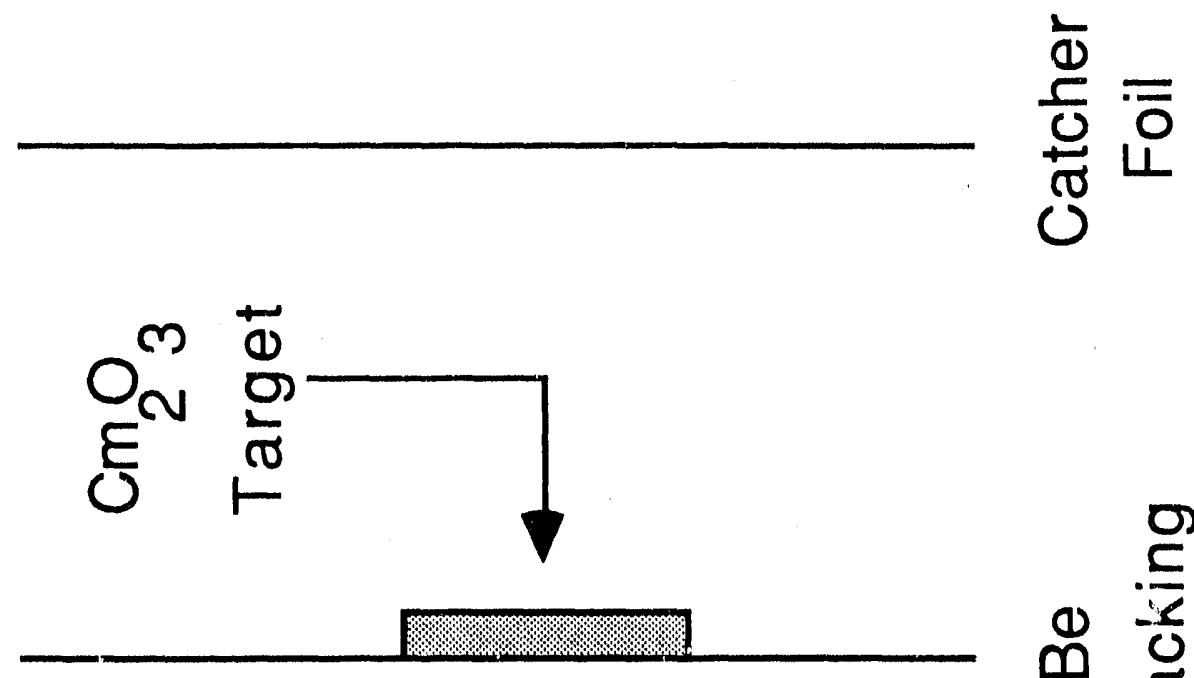

m $\frac{\text { o }}{\infty}$

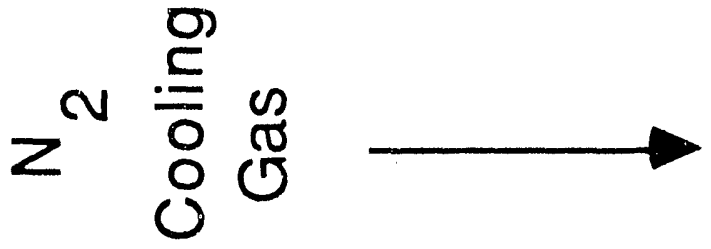

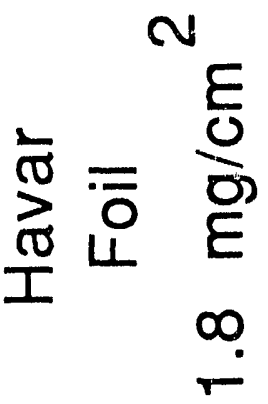

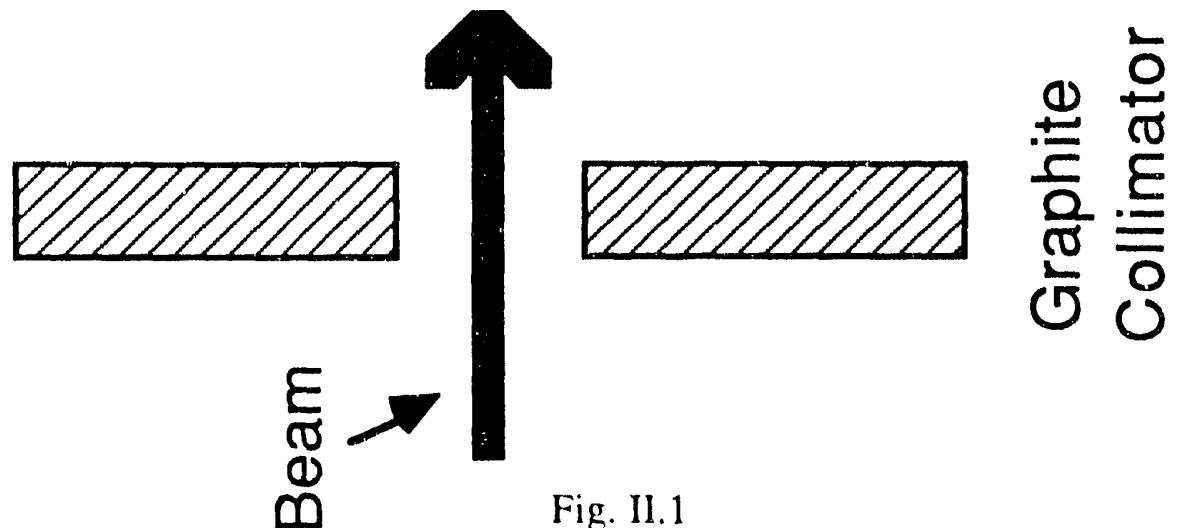

Fig. II.1 


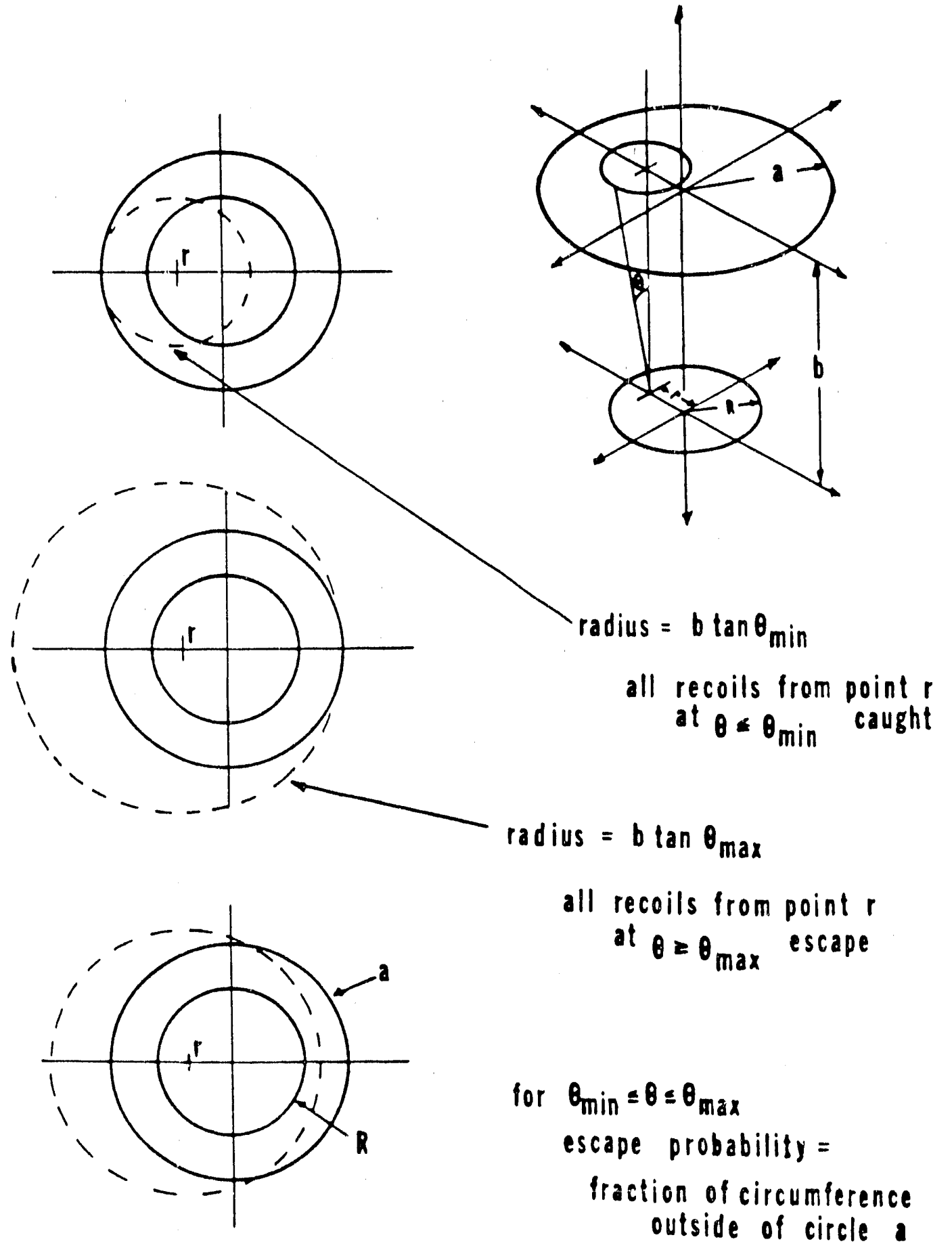

XBL $\quad 836.10249$

Fig. II. 2 


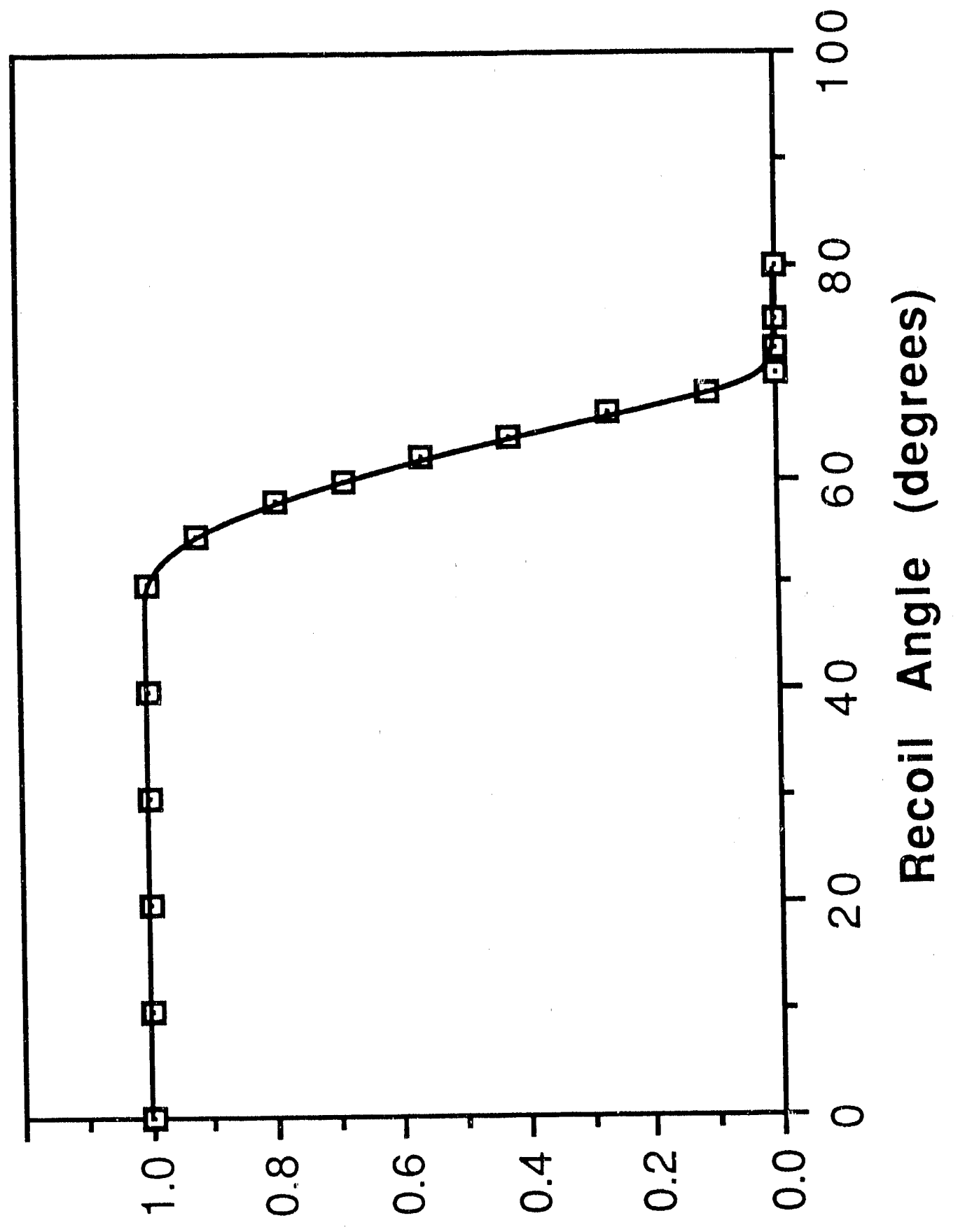

I!osoy 6u!yoleo to "qodd

Fig. II.3 


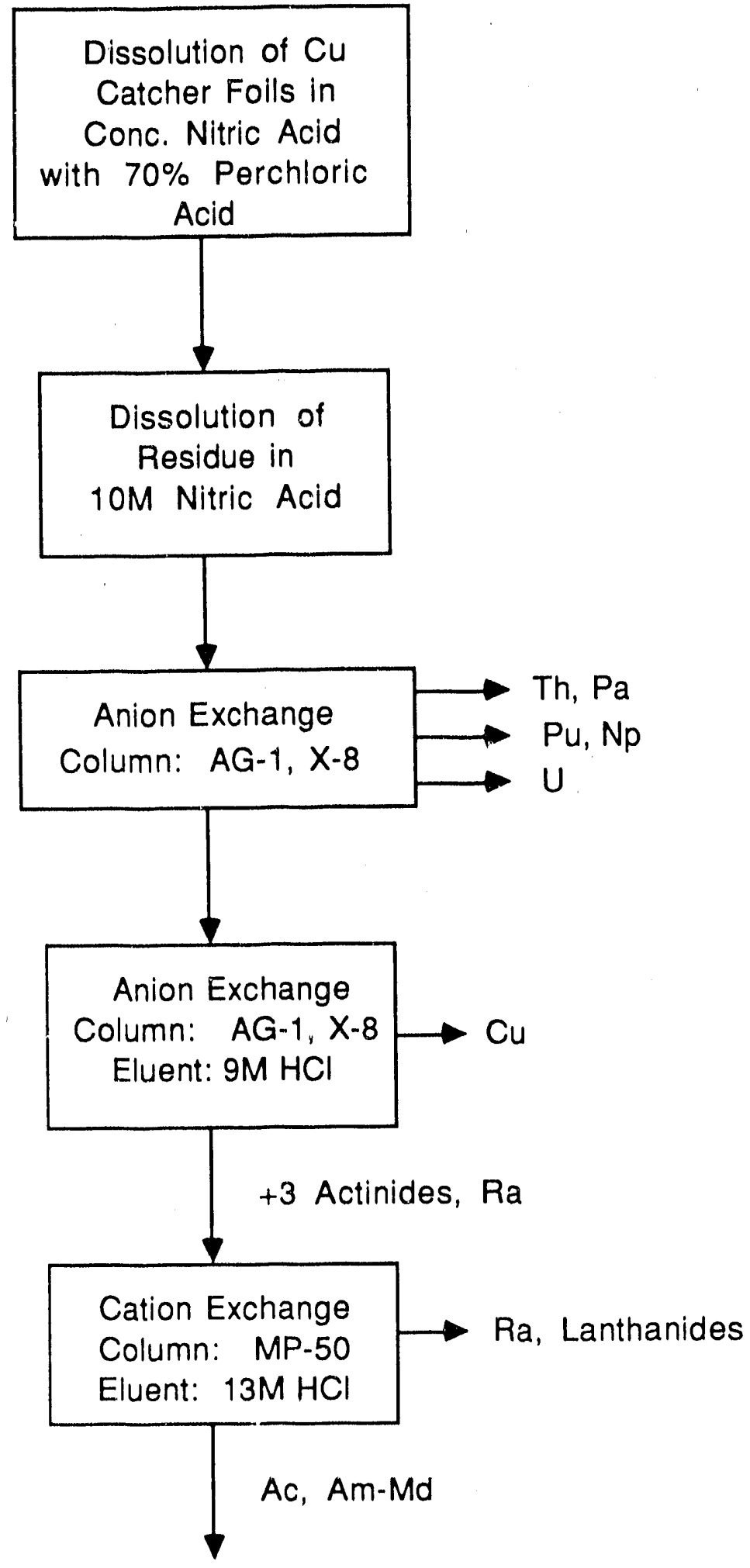

Fig. II.4 


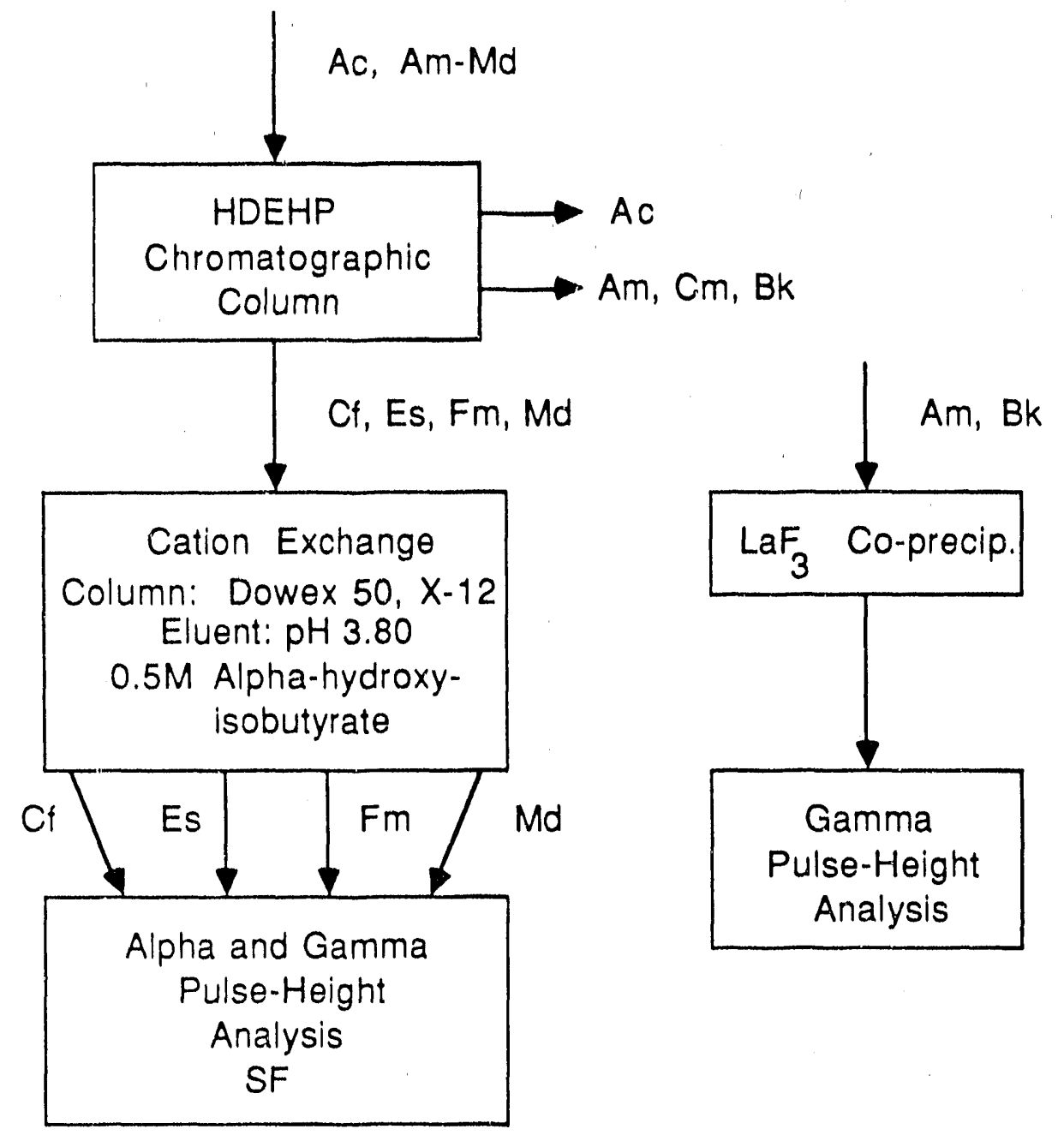

Fig. II.5 


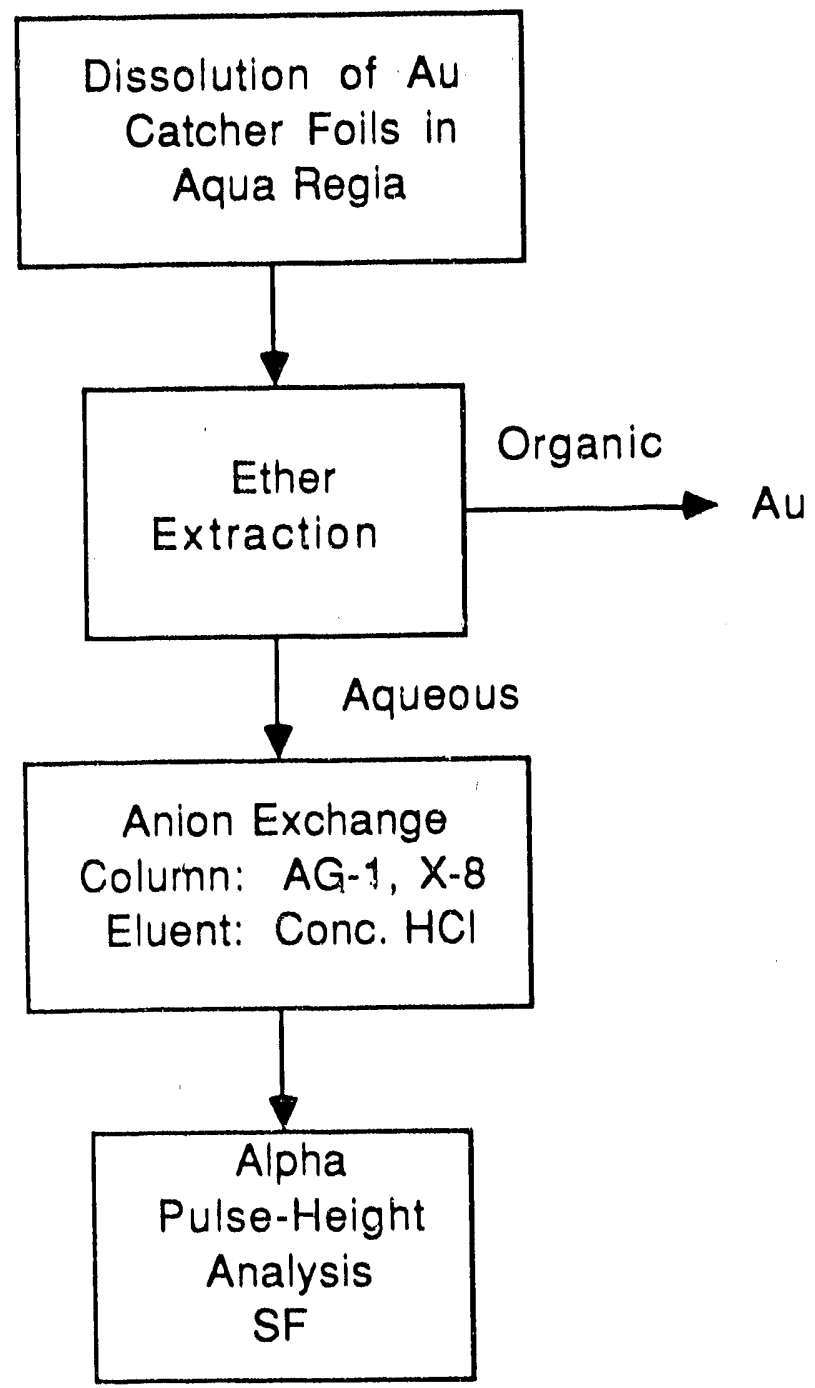

Fig. II.6 


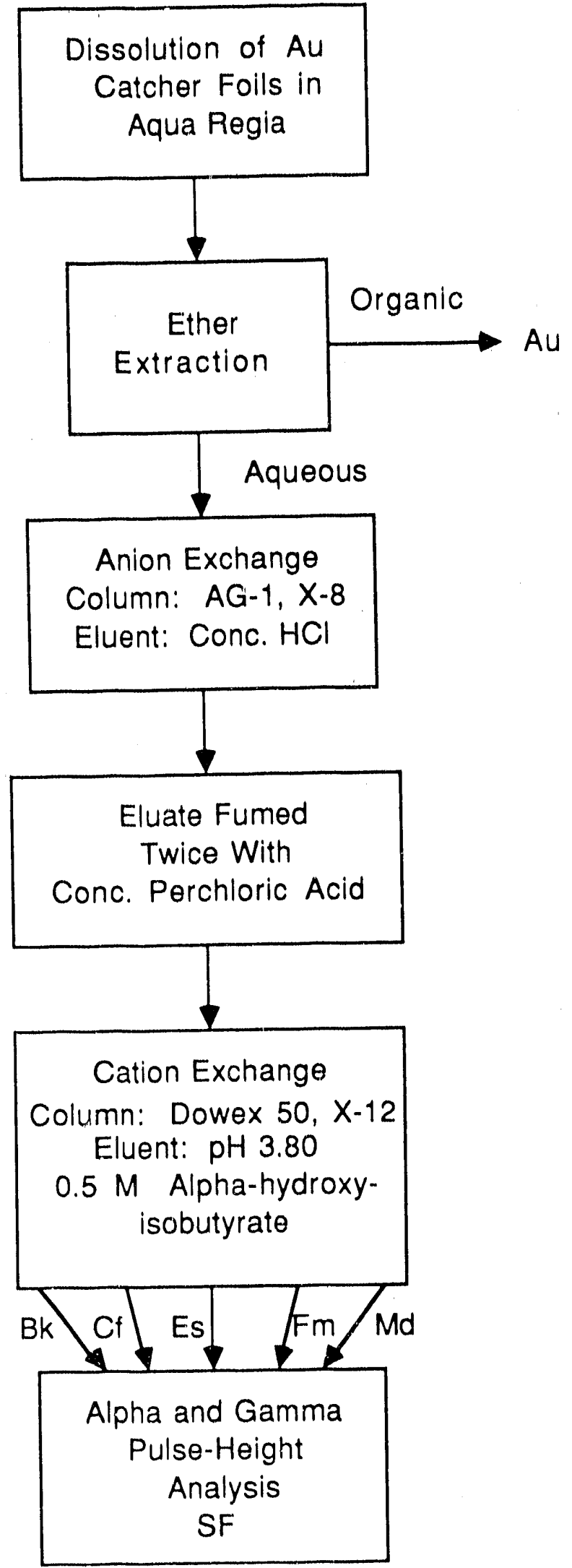

Fig. 11.7 


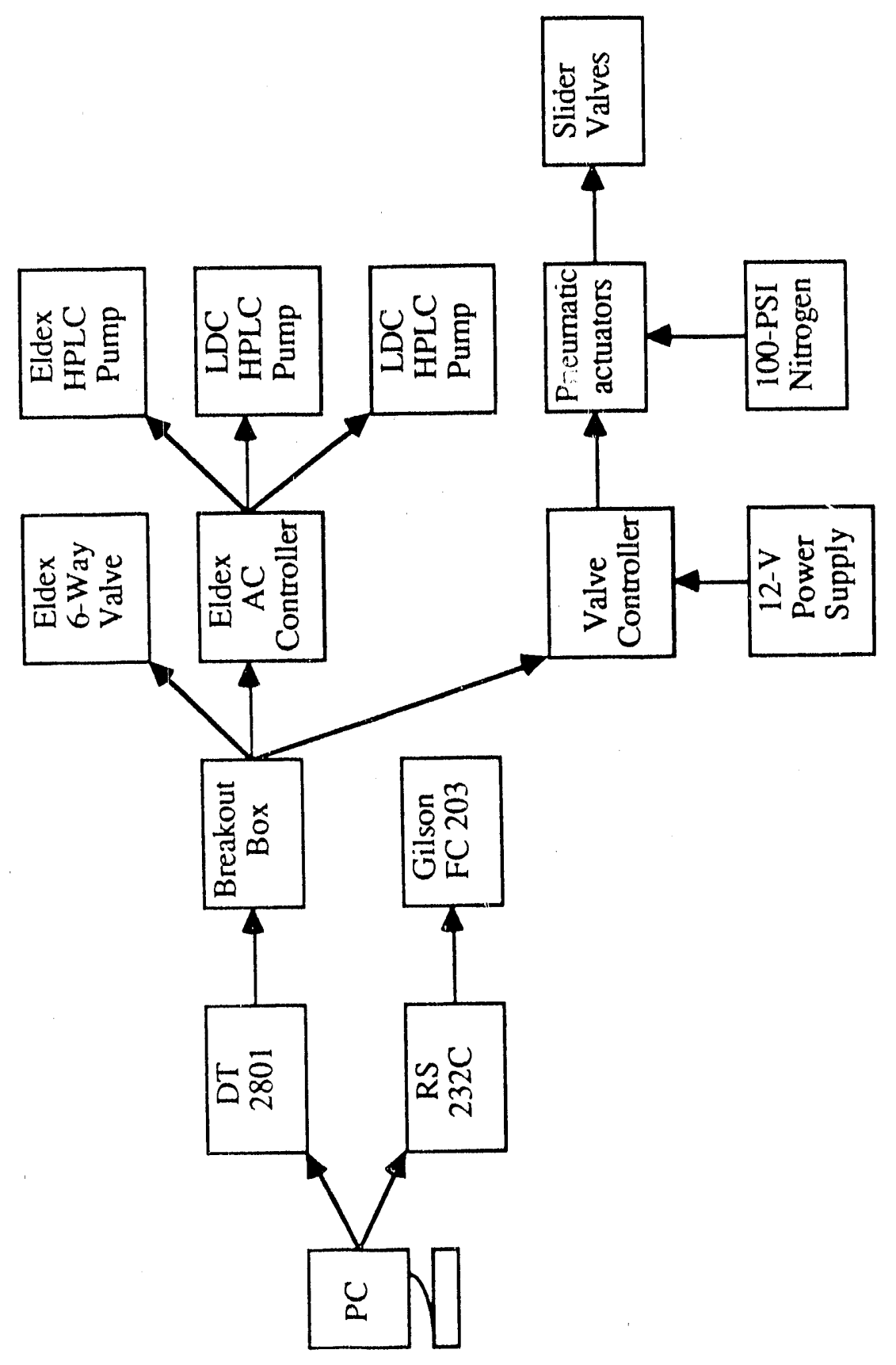

Fig. II. 8 


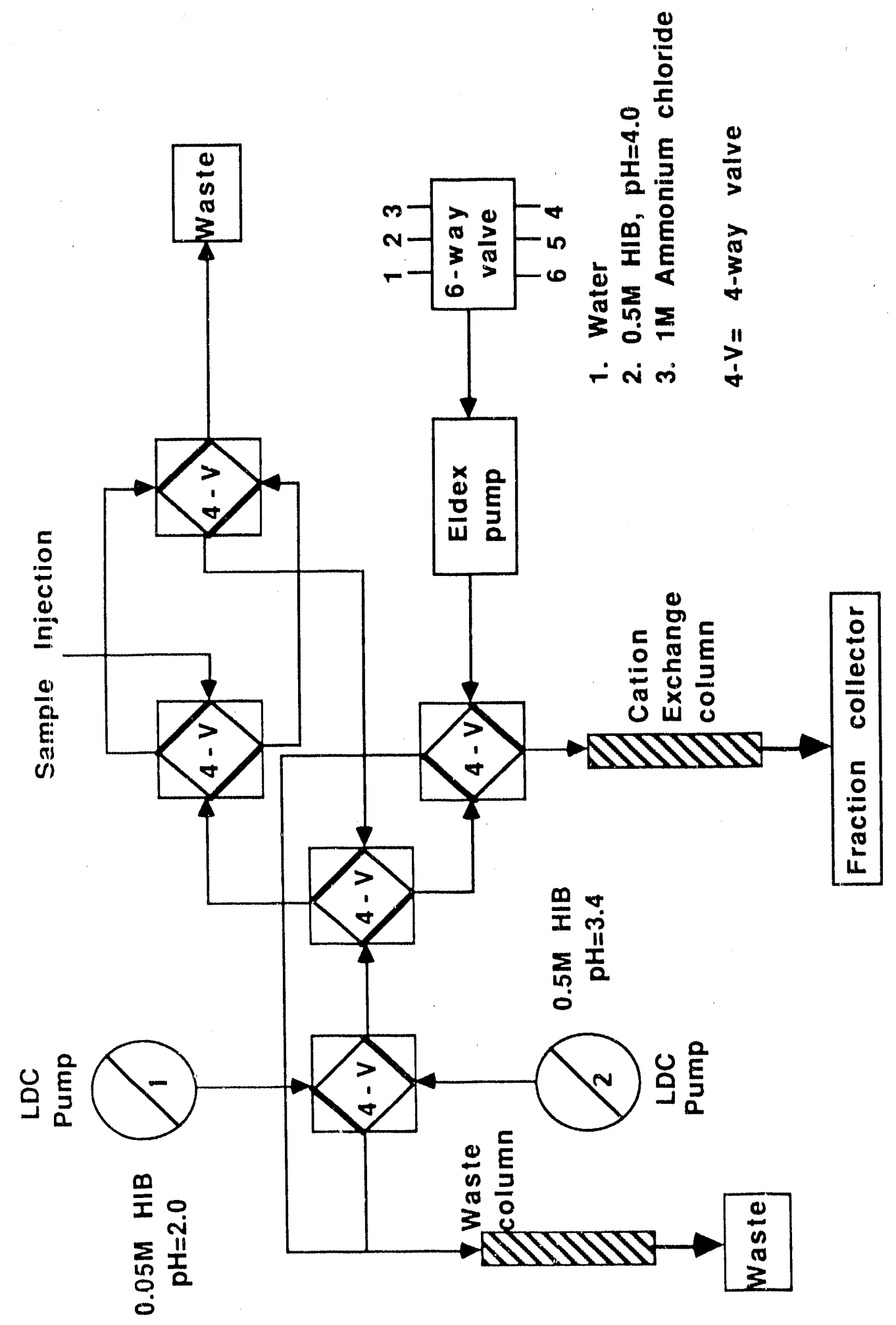

Fig. II.9 


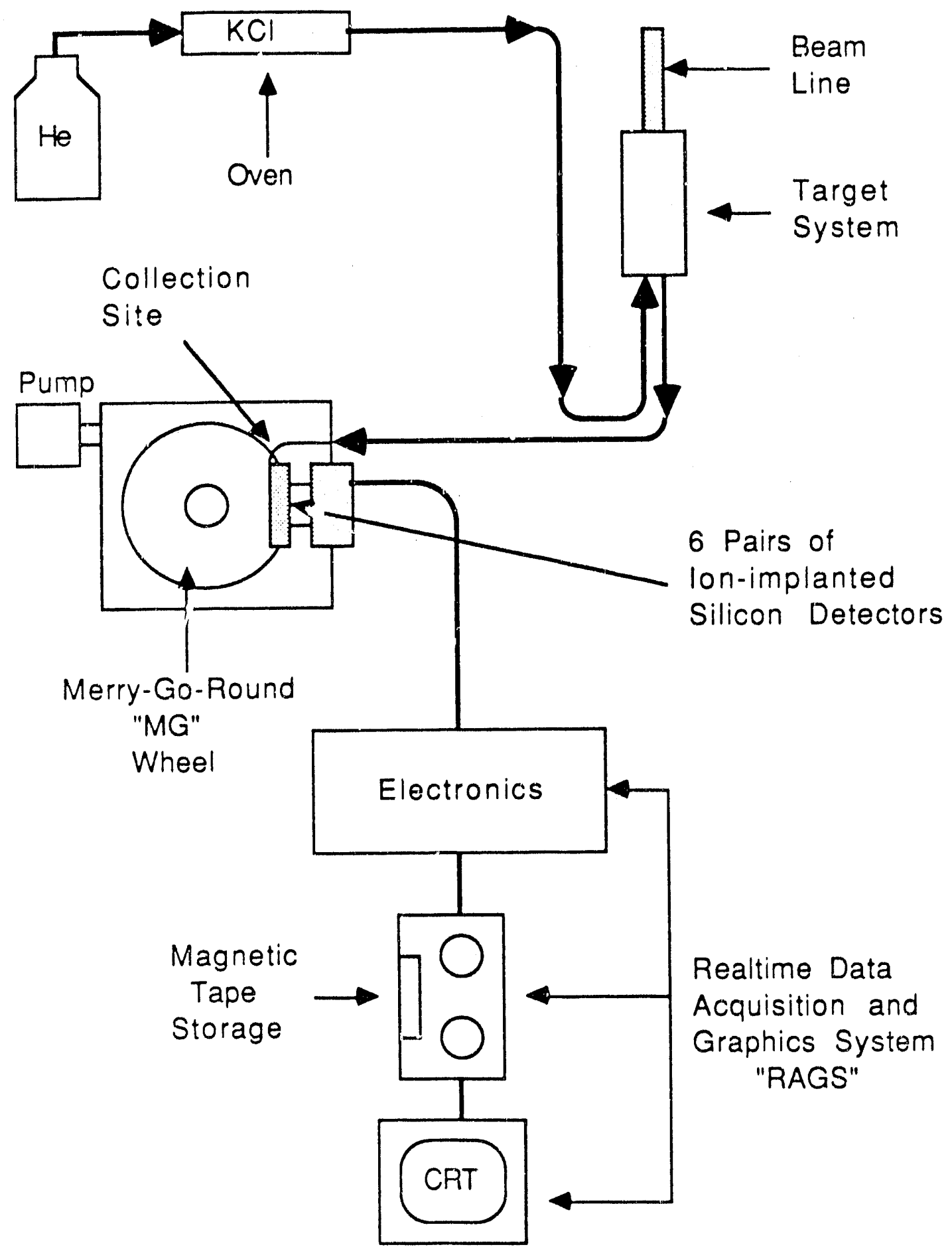

Fig. II.10 


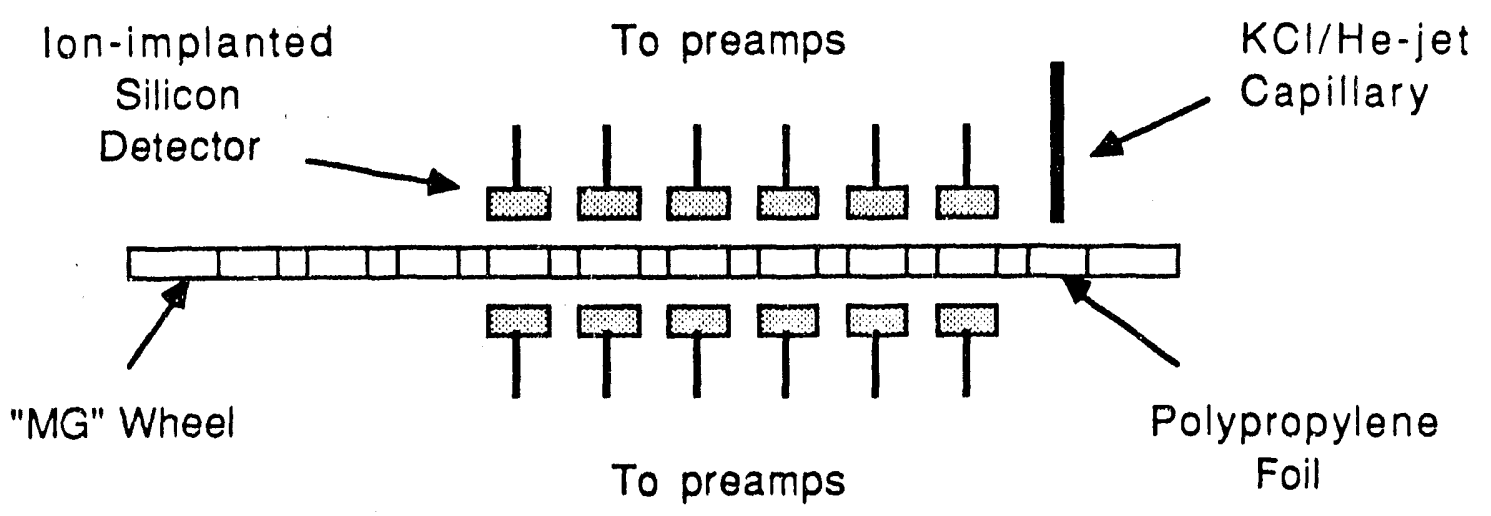

Side View

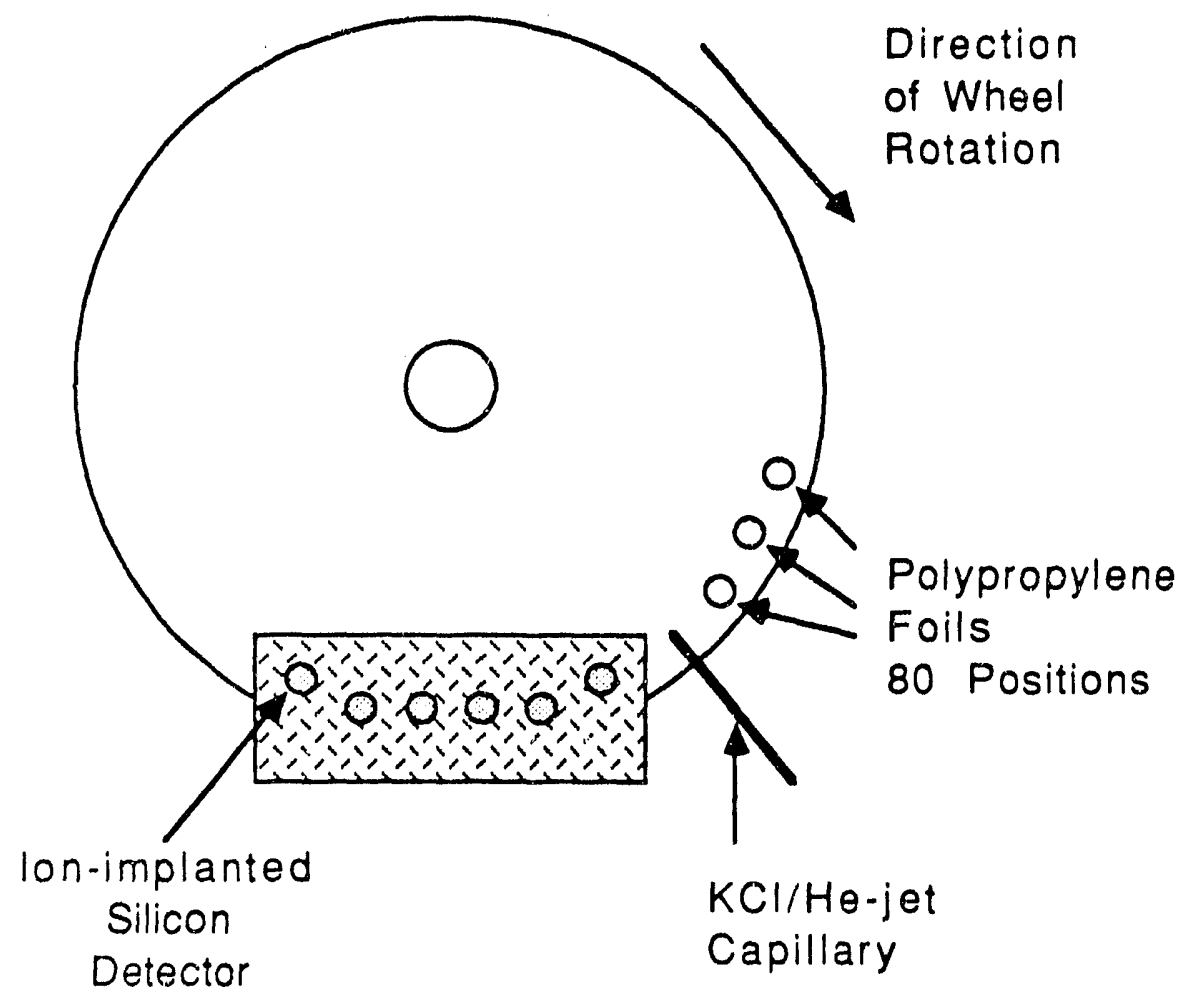

Top View

Fig. II.11 


\section{Alpha-Gamma Counting Chamber}

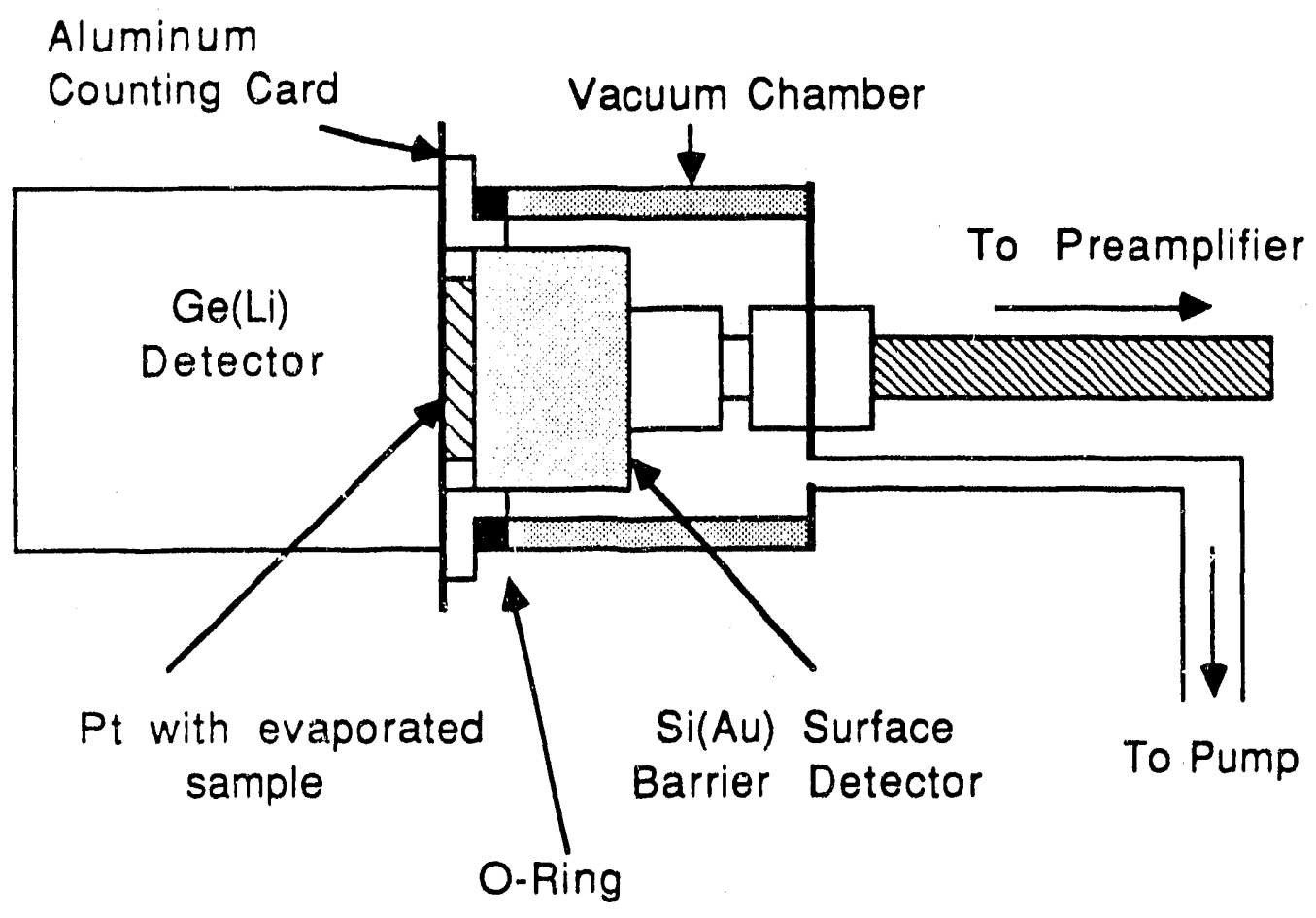

\section{Side View}

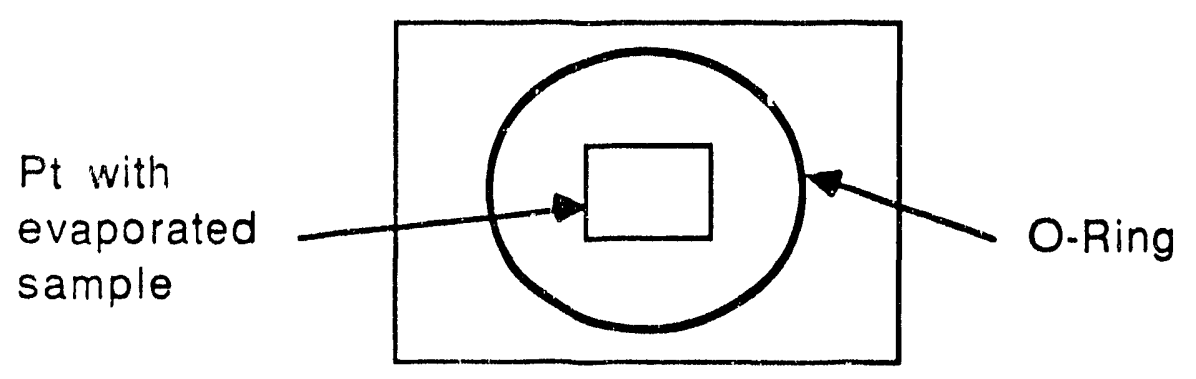

Front View of Counting Card

Fig. II.12 


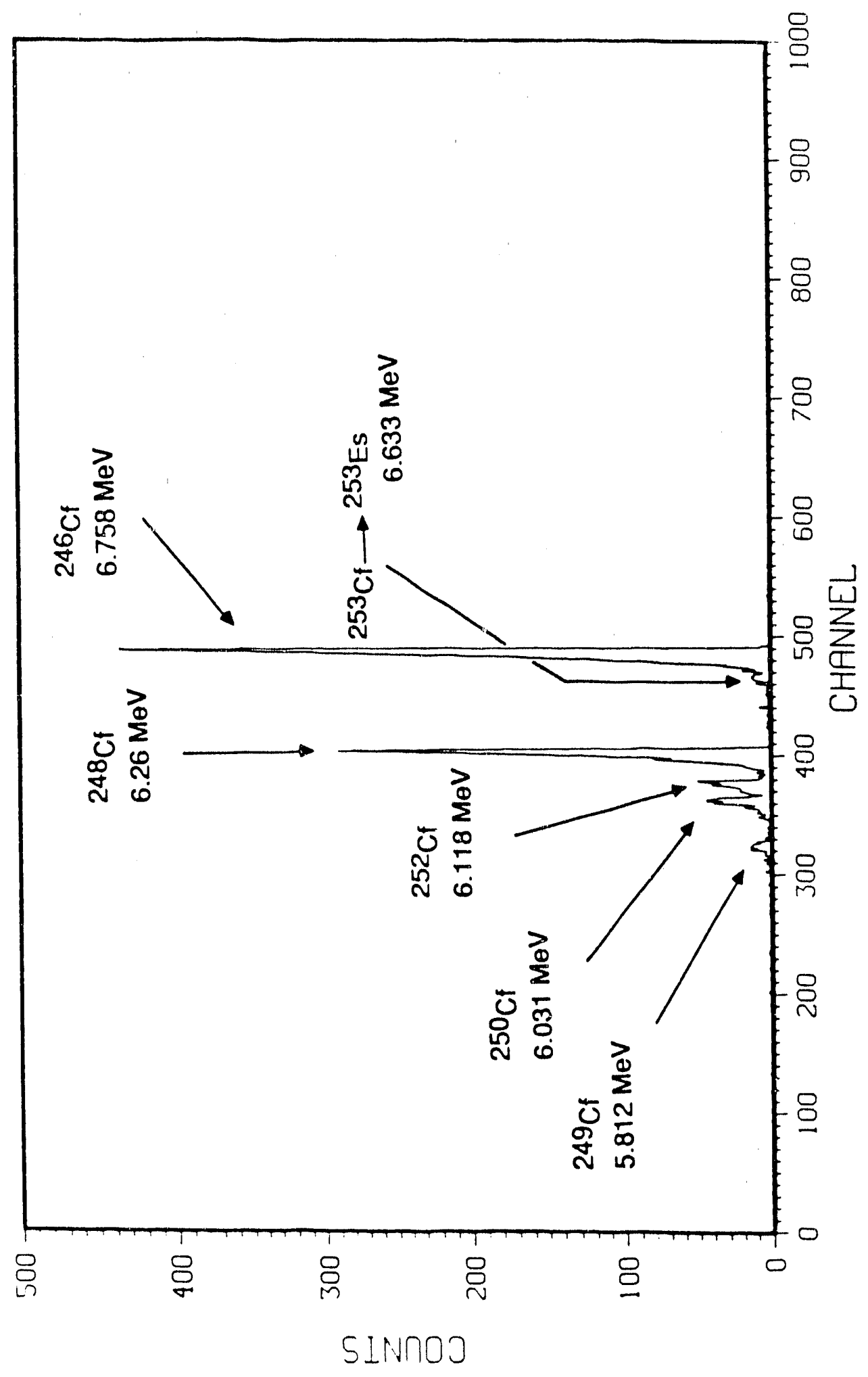

Fig. II.13 


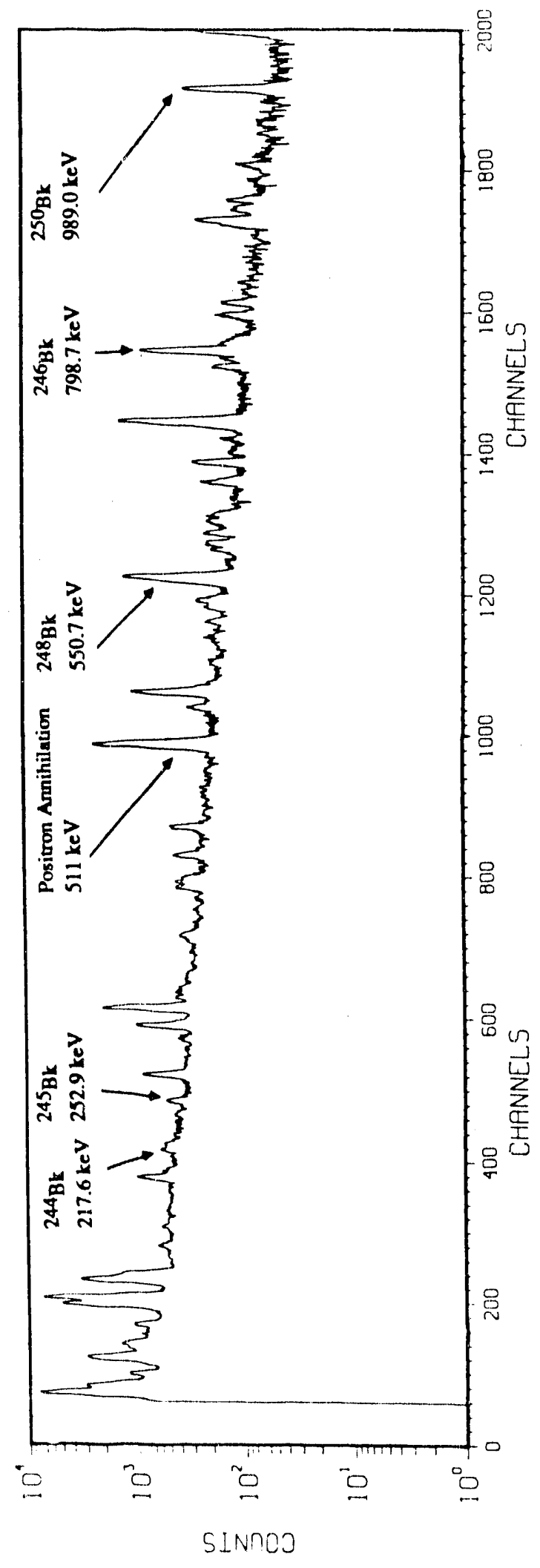

Fig. II.14 


\section{RESULTS AND DISCUSSION}

\section{A. Cross Sections and Excitation Functions}

Theoretically, for the capture of charged particles, the cross section should increase with increasing bombarding energy of the projectile, since the maximum impact parameter, $b_{m}$, for such processes goes as

$$
b_{m}=R\left(1-\frac{V_{c}}{\varepsilon}\right) .
$$

$R$ is the interaction radius, $V_{c}$ is the Coulomb barrier between the target and the projectile, and $\varepsilon$ is the kinetic energy of the projectile. In addition, the cross section for the capture of charged particles can be estimated based upon the geometric cross section of a disk with radius equal to the interaction radius, $R$ :

$$
\sigma_{g e o m}=\pi R^{2}
$$

However for two nuclei to classically interact, $b_{m}<R$. Therefore, an upper limit for the capture of charged particles can be written as

$$
\sigma_{u l}=\pi R^{2}\left(1-\frac{V_{C}}{\varepsilon}\right)=\sigma_{g e o m}\left(1-\frac{V_{c}}{\varepsilon}\right) .
$$

From Eq. 3 it can be seen that the cross section asymptotically approaches the geometric cross section at high $\varepsilon$. However, as more energy is deposited into the system, the probability for particle emission and/or fission increases. Hence, at some higher energy, the decrease in yield due to particle emission and fission should be greater than the increase resulting from the higher bombarding energy. As a result the net production 
cross section for a given nuclide decreases.

Cross sections for nuclides produced from the interactions of ${ }^{12} \mathrm{C},{ }^{31} \mathrm{P},{ }^{40} \mathrm{Ar}$, and ${ }^{44} \mathrm{Ca}$ with ${ }^{248} \mathrm{Cm}$ are listed in Tables III.1-III.5. The energies of the projectiles are given in the lab frame of reference. All cross sections are given in microbarns $(\mu b)$. The statistical standard deviation, $s$, for a given cross section is listed as a percentage of the production cross section. A less than sign $(<)$ in front of the cross section indicates that no activity was detected, therefore an upper limit on the activity was set at the $1 \sigma(68 \%)$ confidence level. In particular, for nonexistent alpha peaks, it was assumed that $<2.3$ counts were seen in the specific counting interval where no activity was detected. For those nonexistent gamma peaks, a formalism developed by Gregorich [GRE85] was used to determine upper limits. This formalism is based upon the definition of chi squared. Hence, the upper limit on the activity of a nuclide at the end of bombardment is given by

$$
A_{O}=\sum_{i=1}^{N} \frac{\exp \left(-2 \lambda t_{i}\right)}{\sigma_{i}^{2}}
$$

where $\lambda$ is the decay constant, $\sigma_{i}$ is the error in the background count rate of the $i$ th interval, and $t_{i}$ is the time between the end of bombardment and the $i$ th interval.

A given nuclide can be produced directly via a transfer reaction or indirectly by the decay of a parent nuclide produced in the bombardment. Hence, in order to obtain the cross section for the transfer reaction, the amount of the nuclide produced via the indirect route(s) must be subtracted from the amount produced directly. Therefore, corrections have been made, where appropriate, to the cross sections of the daughter ruclides from the decay of radioactive parent nuclides during the bombardment and the time interval between the end of bombardment and chemical separation. For example, the decay of $248 \mathrm{Bk}^{m}\left(t_{1} / 2=23.7 \mathrm{~h}\right)$ to ${ }^{248} \mathrm{Cf}\left(t_{1 / 2}=334 \mathrm{~d}\right)$ represents a significant 
correction $(\cong 25 \%)$ to the amount of $248 \mathrm{Cf}$ produced directly via a transfer reaction.

Only the relative intensities are known for the gamma rays from ${ }^{244} \mathrm{Bk}$. However, by assuming the electron capture branch to be $100 \%$, and the fluorescence yield to be 0.978 , and knowing the relative intensities of the $\mathrm{Cm} x$-rays, the absolute intensity of the 217.6 $\mathrm{keV}$ gamma ray was estimated to be $37.4 \pm 12 \%$ [BRO78]. The decay scheme of ${ }^{251} \mathrm{Bk}$ has not been measured, therefore the cross sections for this nuclide are based upon the assumption that the absolute intensity of the most abundant gamma ray is $100 \%$; therefore, these values are lower limits only.

The cross sections plotted for ${ }^{248} \mathrm{Bk}$ and ${ }^{254} \mathrm{Es}$ are in reality only lower limits due to the fact that there were unobserved isomers. The effects of these unobserved isomers on the mass-yield curves are discussed in section III.G.c.

\section{B. Excitation Energies}

Hoffman and Hoffman [HOF82] have developed a formalism to calculate the energy available for product excitation in transfer reactions. This model is based upon a binarytype reaction in which the target and projectile exchange nucleons but do not amalgamate into a compound nucleus. The center of mass bombarding energy, $E_{c m}$, of the projectile is given by

$$
E_{c m}=E_{b}(i)+K E(i)=E_{b}(f)+K E(f)+K E(R)-Q_{g g}+E_{x},
$$

where $E_{b}(i)$ and $E_{b}(f)$ are the entrance channel and exit channel Coulomb barriers respectively, $K E(i)$ is the residual kinetic energy of the projectile (the amount of energy over the entrance channel Coulomb barrier), $K E(R)$ is the rotational kinetic energy of the target-like fragment, $Q_{g g}$ is the ground state $Q$ value of the reaction channel (mass of the reactants - mass of the products), and $E_{X}$ is the excess energy available for product excitation (excitation energy).

$K E(f)$ was calculated assuming that the kinetic energy per nucleon of the projectile- 
like fragment is the same as the residual kinetic energy per nucleon of the projectile at the point of contact with the target, assuming two touching spheres.

Hence

$$
K E(f)=[K E(i)] \frac{A(f)}{A(i)}=\left[E_{c m}-E_{b}(i)\right] \frac{A(f)}{A(i)},
$$

where $A(i)$ is the mass of the projectile and $A(f)$ is the mass of the projectile-like fragment. Since this model was developed for reactions at energies near the entrance channel Coulomb barrier $\left(E_{c m} \cong E_{b}(i)\right), K E(f)$ and $K E(R)$ are always small in comparison to $E_{x} . K E(R)$ was calculated using

$$
K E(R)=\frac{\hbar^{2}[J(J+1)]}{2 !},
$$

where $J$ is the rotational angular momentum, and $I$ is the nuclear moment of inertia. For these calculations I was set equal to one half the moment of inertia of a rigid sphere.

If $E_{X}$ is negative, an energy barrier must be overcome for the reaction to proceed with a high probability. Reactions with negative excitation energies should therefore display excitation functions with maxima at some energy above the Coulomb barrier. The cross sections should then decrease at higher projectile kinetic energy as fission or particle emission becomes energetically possible. For reactions with positive excitation energies (of the order of neutron binding energies or fission barriers) the excitation function should peak at or near the Coulomb barrier and decrease with increasing projectile kinetic energy. $E_{x}$ 's have been calculated for several target-like and projectile-like fragments from the ${ }^{12} \mathrm{C},{ }^{31} \mathrm{P},{ }^{40} \mathrm{Ar},{ }^{40} \mathrm{Ca},{ }^{44} \mathrm{Ca}$, and ${ }^{48} \mathrm{Ca}$ systems and are given in Tables III.6-III.10. 


\section{Division of Energy}

Lee et al. [LEE83] estimated the fraction, $F_{t}$, of the projectile kinetic energy transformed into target-like fragment excitation energy using the equation

$$
F_{t}=\frac{E_{f, n}-E_{x}}{E_{M}-V_{c}},
$$

where $E_{f, n}$ is the height of the fission barrier [BRI79] [BRI80] or the neutron binding energy [SEE67] in $\mathrm{MeV}$ whichever is lower, $E_{x}$ is the excitation energy described earlier, $E_{M}$ is the maximum in the experimental excitation function, and $V_{C}$ is the calculated Coulomb barrier. This formula assumes that the maximum in the excitation function occurs when the product excitation energy is comparable to the fission barrier or neutron binding energy. This calculation in not applicable when the reaction energy is greater than the neutron binding energy or the fission barrier, because enough energy is already available to cause depletion of the cross section by fission or neutron emission. The calculated $F_{t}$ values for the ${ }^{12} \mathrm{C},{ }^{31} \mathrm{P},{ }^{40} \mathrm{Ar}$, and ${ }^{44} \mathrm{Ca}$ systems are given in Tables III.11-III.14. $E_{t}$, the fraction of energy transferred assuming energy transfer is proportional to mass transfer, is also shown in Tables III.11-III.14. E $E_{t}$, which can appear as excitation energy of the heavy product, was calculated using the formula

$$
E_{t}=\frac{M}{A} \text {, }
$$

where $M$ is the number of nucleons transferred and $A$ is the mass number of the projectile. $F_{t}$ and $E_{t}$ valut, for the actinides produced in the ${ }^{12} \mathrm{C},{ }^{31} \mathrm{P},{ }^{40} \mathrm{Ar}$, and ${ }^{44} \mathrm{Ca}$ systems are discussed with each individual system in the following sections. 


\section{D. ${ }^{12} \mathrm{C} \cdot 248 \mathrm{Cm}$ System}

Production cross sections for the measured isotopes of Bk, Cf, Es, and Fm from the interactions of ${ }^{12} \mathrm{C}$ with ${ }^{248} \mathrm{Cm}$ along with the statistical standard deviation, $s$, are shown in Table III.1. The measured production cross sections for some Md and No isotopes are given in Table III.2. The calculated Coulomb barrier is $72 \mathrm{MeV}$ in the lab system. Ghiorso et al. [GHI67] measured production cross sections for ${ }^{255} \mathrm{No},{ }^{256} \mathrm{No}$, and $257 \mathrm{No}$ at $71 \mathrm{MeV}$ from the ${ }^{12} \mathrm{C}-248 \mathrm{Cm}$ system. They obtained values of 380,740 , and $80 \mathrm{nb}$ respectively for $255 \mathrm{No}, 256 \mathrm{No}$, and $257 \mathrm{No}$, versus $21.5,203$, and $33.2 \mathrm{nb}$ obtained in the present study. Hoffman et al. [HOF90] have also measured the ${ }^{248} \mathrm{Cm}\left({ }^{12} \mathrm{C}, 4 n\right)^{256}$ No cross section at $71 \mathrm{MeV}$ and obtained a value of $250 \mathrm{nb}$, in agreement with the value of this work. The discrepancy between Ghiorso's values and those in this thesis are not understood.

\section{Isotopic Distributions}

The isotopic distributions for $\mathrm{Bk}, \mathrm{Cf}, \mathrm{Es}, \mathrm{Fm}$, and No from the interactions of 84$\mathrm{MeV}\left(1.16 \mathrm{X}\right.$ Coulomb barrier) ${ }^{12} \mathrm{C}$ ions with ${ }^{248} \mathrm{Cm}$ are shown in Fig. III.1. This energy was chosen so that this system could be compared to other systems at the same energy relative to the entrance channel Coulomb barrier. All of the data points for each element are connected with lines. These lines are included only to aid the eye in viewing the plot. The mass yield curves have maxima at $248 \mathrm{Bk},{ }^{250} \mathrm{Cf},{ }^{251} \mathrm{Es},{ }^{253} \mathrm{Fm}$, and

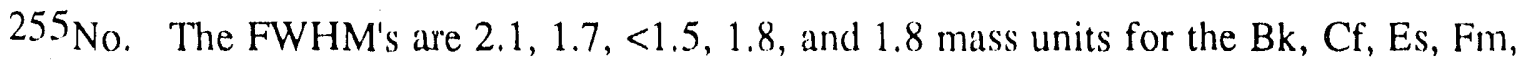
and No curves, respectively. Since neither ${ }^{25()}$ Es nor ${ }^{249}$ Es were seen at this energy, only an upper limit can be set on the width of the Es curve.

Isotopic distributions for $\mathrm{Bk}, \mathrm{Cf}, \mathrm{Es}, \mathrm{Fm}, \mathrm{Md}$, and No from the interactions of 71$\mathrm{MeV}\left(0.98 \times\right.$ Coulomb barrier) ${ }^{12} \mathrm{C}$ ions with ${ }^{248} \mathrm{Cm}$ are plotted in Fig. III.2. The curves have FWHM's of 1.7, 1.6, <1.0, 1.3, 1.0, and 1.0 mass units for Bk, Cf, Es, Fm, $\mathrm{Md}$, and No respectively. The widths of these isotopic distributions are narrower than the corresponding widths at $1.16 \times$ Coulomb barrier. 


\section{Excitation Functions}

\section{a. Excitation Functions for Bk Isotopes}

The excitation functions for the Bk isotopes 245, 246, 248, and 250 are displayed in Fig. III.3. The lines connecting the experimental cross sections for a given isotope are included only to aid the eye. Assuming a binary-type transfer mechanism, the production of all of the Bk isotopes requires the transfer of one proton from the projectile to the target, along with the transfer of various numbers of neutrons to or from the target. For example ${ }^{248} \mathrm{Bk}^{m}$ requires the transfer of only one neutron whereas the production of ${ }^{244} \mathrm{Bk}$ requires a five neutron transfer from the target to the projectile. Therefore, the isotope requiring the transfer of the fewest number of nucleons for which $E_{X}$ is positive (but not large enough to cause fission or neutron emission) should have the highest cross section. ${ }^{249} \mathrm{Bk}$ meets these requirements. This nuclide requires the transfer of just one proton from the projectile to the target and has an $E_{x}$ of $-0.7 \mathrm{MeV}$. However, the ${ }^{249} \mathrm{Bk}$ cross sections were not included for two reasons. First of all ${ }^{249} \mathrm{Bk}$ is used as both a tracer and a target in our laboratory and secondly its measurement is extremely difficult. Since ${ }^{249} \mathrm{Bk}$ is used in our laboratory, contamination is a possibility that has to be ansidered. The difficulty in measurement stems from the fact that the absolute intensities of the most abundant gamma ray and alpha branch from ${ }^{249} \mathrm{Bk}$ are on the order of $1 \times 10^{-5}$. Since ${ }^{249} \mathrm{Bk}$ has a $99+\% \mathrm{~B}^{-}$branch, its activity can be determined from the growth of the ${ }^{249} \mathrm{Cf}$ daughter. However, the long half-life of ${ }^{249} \mathrm{Cf}\left(t / \frac{1}{2}=351\right.$ y) makes the detection of this nuclide difficult also. From the experimental data it can be seen that $248 \mathrm{Bk}^{m}$ has the highest cross sections over the energy range studied followed by $250_{\mathrm{Bk}}, 246_{\mathrm{Bk}}$, and $245_{\mathrm{Bk}}$. The production of both $248 \mathrm{Bk}^{m}$ and $250_{\mathrm{Bk}}$ requires the transfer of two nucleons between the target and projectile. However, the $E_{X}$ value of ${ }^{248} \mathrm{Bk} \cdot \mathrm{m}$ is $-3.5 \mathrm{MeV}$ whereas ${ }^{250} \mathrm{Bk}$ has an $E_{x}$ of $-7.6 \mathrm{MeV}$; hence, the higher

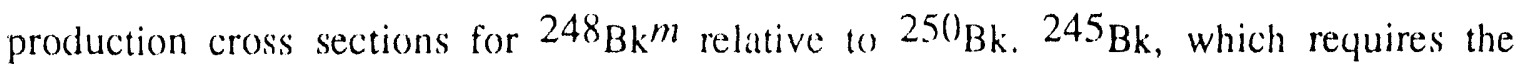
transfer of five nucleons has lower production cross sections than ${ }^{246} \mathrm{Bk}$ (requiring the 
transfer of four nucleons) over the energy range studied. Since the excitation functions are increasing with increasing bombarding energy, they are consistent with their calculated negative $E_{x}$ values.

\section{b. Excitation Functions for Cf lsotopes}

Fig. III. 4 displays the excitation functions for ${ }^{246} \mathrm{Cf},{ }^{248} \mathrm{Cf},{ }^{250} \mathrm{Cf},{ }^{252} \mathrm{Cf}$, and ${ }^{253} \mathrm{Cf}$. As can be seen from this figure, ${ }^{250} \mathrm{Cf}$, the isotope requiring the transfer of the fewest nucleons between the target and projectile, has the highest production cross sections. Two of the isotopes, 250 and 252 , have positive $E_{X}{ }^{\prime}$ s and display excitation functions which have maxima near the Coulomb barrier, consistent with their calculated $E_{x}{ }^{\prime}$ 's of 4.9 and $6.9 \mathrm{MeV} .{ }^{246} \mathrm{Cf},{ }^{248} \mathrm{Cf}$, and ${ }^{253} \mathrm{Cf}$ each have negative $E_{x}{ }^{\prime}$ 's with excitation functions consistent with this fact.

\section{c. Excitation Functions for Es Isotopes}

The excitation functions measured for the Es isotopes 251,252, 253,254m, and 255 are plotted in Fig. III.5. The isotope requiring the transfer of the fewest nucleons, 251, has the highest cross sections at two energies, with 2.52 having the highest cross sections at the other three energies. ${ }^{255} \mathrm{Es}$, the isotope requiring the transfer of the most nucleons (seven), has the lowest cross sections. The shapes of the excitation functions of these Es isotopes are consistent with their calculated positive $E_{X}$, values with the possible exception of $251_{\text {Ess. No }}{ }^{251} \mathrm{Es}$ was found at $71 \mathrm{MeV}$; therefore, only an upper limit could be set on the cross section.

\section{d. Excitation Functions for Fm Isotopes}

Fig. III.6 shows the excitation functions for the Fm isotopes 252, 253, 254, 255, and 256. ${ }^{253} \mathrm{Fm}$, which requires the transfer of five nucleons has the highest cross sections at three energies, followed by $252 \mathrm{Fm}, 254 \mathrm{Fm}, 25.5 \mathrm{Fm}$, and finally $256 \mathrm{Fm}$. It should be noted that even though $252 \mathrm{Fm}$ requires the transfer of only four nucleons, it has lower production cross sections than $253 \mathrm{Fm}$ at the three lower energies. The higher cross 
sections for $253 \mathrm{Fm}$ could be due to the greater $E_{x}$ value of this nuclide $(5.7 \mathrm{MeV})$ as compared to $252 \mathrm{Fm}\left(E_{x}=3.0 \mathrm{MeV}\right)$. This would explain the lower cross sections of $253 \mathrm{Fm}$ at the two higher energies. Since more energy would be available for fission and neutron evaporation, the cross section for $25.3 \mathrm{Fm}$ should diminish faster than the cross section for $252 \mathrm{Fm}$. $252 \mathrm{Fm}$ has an excitation function shape that is not consistent with its calculated positive $E_{x}$ value. Likewise, the shape of $256 \mathrm{Fm}$ 's excitation function is consistent with a negative $E_{x}$ value, not its very positive value of $22.4 \mathrm{MeV}$.

e. Ft and $E_{t}$ Vulues

$F_{t}$ and $E_{t}$ values for the ${ }^{12} \mathrm{C}$ system are shown in Table 111.11 . The $F_{t}$ 's range in value from 0.08 to 0.72 . No trends can be seen in the $F_{t}$ values and no correlations between $F_{t}$ and $E_{t}$ are apparent. In general, however, $E_{t}$ is less than $F_{t}$, indicating that the fraction of energy transferred is greater than the fraction of mass transferred.

\section{E. 31 P. $248 \mathrm{Cm}$ System}

The production cross sections along with the statistical standard deviation, $s$, for the measured isotopes of $\mathrm{Bk}$, Cf, Es, and Fm from the interactions of $31 \mathrm{P}$ with ${ }^{248} \mathrm{Cm}$ are listed in Table III.3.

\section{Isotopic Distributions}

Isotopic distributions for Bk, Cf, Es, and Fm produced from the interactions of $207-$ MeV (1.17 X Coulomb barrier) $31 \mathrm{P}$ ions with $248 \mathrm{Cm}$ are plotted in Fig. III.7. The isotopic distributions have maxima at $248 \mathrm{Bk} m, 250 \mathrm{Cf},{ }^{251} \mathrm{Es}$, and $252 \mathrm{Fm}$. All of these nuclides correspond to the isotope requiring the transfer of the fewest number of nucleons for a given element, except for $248 \mathrm{Bk}^{\prime \prime}$. All of the curves appear to be symmetric with full-widths-at-half-maximum (FWHM) of $2.5,2.5,<1.5$, and 2.3 mass units for Bk, Cf, Es, and Fm respectively. Since only upper limits were set on the ${ }^{249}$ Es, and 25$)^{2}$ Es cross sections, only an upper limit can be set on the FWHM of the Es curve. Additionally, the maxima and the widths of the mass-yield curves stay constant over the energy range investigalted. 


\section{Excitation Functions}

\section{a. Excitation Functions for Bk Isotopes}

Excitation functions for the $\mathrm{Bk}$ isotopes $244,245,246,248 \mathrm{~m}$, and 250 are shown in Fig. III.8. ${ }^{248} \mathrm{Bk}^{m}$ has the highest cross sections at all energies, with ${ }^{246} \mathrm{Bk}$ next, followed by ${ }^{250} \mathrm{Bk}, 245 \mathrm{Bk}$, and finally $244 \mathrm{Bk}$. All of the $\mathrm{Bk}$ excitation functions have naxima at about $207 \mathrm{MeV}$. The shapes of the excitation functions of ${ }^{244} \mathrm{Bk}$ and ${ }^{248} \mathrm{Bk}^{\prime \prime \prime}$ are consistent with their positive excitation energies. ${ }^{245} \mathrm{Bk},{ }^{246} \mathrm{Bk}$, and ${ }^{250} \mathrm{Bk}$ have excitation functions which are very similar in shape. They increase by about a factor of three between 174 and $207 \mathrm{MeV}$ and then show a decrease by more than a factor of two over the next $32 \mathrm{MeV}$. The shapes of the excitation functions of ${ }^{245} \mathrm{Bk}$ and ${ }^{246} \mathrm{Bk}$, however, do not appear to be consisient with their calculated positive excitation energies. Since their $E_{X}{ }^{\prime} s$ are positive, one would not expect an increase in the cross sections at the lower bombarding energies

\section{b. Excitation functions for Cf isotopes}

Excitation functions for the various Cf isotopes measured in this study are shown in Fig. III.9. ${ }^{250} \mathrm{Cf}$ has the highest cross sections, followed by ${ }^{248} \mathrm{Cf},{ }^{246} \mathrm{Cf},{ }^{252} \mathrm{Cf}$, and finally ${ }^{253} \mathrm{Cf} .{ }^{250} \mathrm{Cf}$ requires the transfer of the fewest number of nucleons, two protons, and additionally it hes an $E_{x}$ of $7.2 \mathrm{MeV}$. ${ }^{248} \mathrm{Cf}$ has much higher cross sections than ${ }^{252} \mathrm{Cf}$ even though both require the transfer of four nucleons. In fact, ${ }^{252} \mathrm{Cf}$ can be produced by the transfer of an alpha particle from the projectile to the target. However, ${ }^{248} \mathrm{Cf}$ has an $E_{X}$ of $8.8 \mathrm{MeV}$ while ${ }^{252} \mathrm{Cf}$ has an $E_{X}$ of only $0.6 \mathrm{MeV}$. This could explain the lower yields of ${ }^{252} \mathrm{Cf}$ relative to ${ }^{248} \mathrm{Cf}$. The ${ }^{248} \mathrm{Cf},{ }^{250} \mathrm{Cf}$, and ${ }^{252} \mathrm{Cf}$ excitation functions have similar shapes, consistent with their positive $E$ 's. However, for ${ }^{253} \mathrm{Cf}$, the excitation function at the Coulomb barrier is near its maximum and decreases with increasing projectile energy, inconsistent with its $E_{X}$ of $-8.1 \mathrm{MeV} .{ }^{246} \mathrm{Cf}$, with an $E_{X}$ of $6.9 \mathrm{MeV}$, has an excitation function which is consistent with a nugative $E_{X}$ vaiue. 


\section{c. Excitation functions for Es isotopes}

Fig. III.10 shows excitation functions for the Es isotopes 251, 252, 253, 254, and 255. The highest cross sections occur for ${ }^{251} \mathrm{Es}$, the isotope requiring the transter of the fewest number of nucleons (three protons). As the number of nucleons transferred increases, the cross sections decrease. In addition, the shapes of the excitation functions are consistent with their calculated excitation energies.

\section{d. Excitation functions for Fm isotopes}

Excitation functions for several isotopes of Fm are shown in Fig. III.11. The shapes of the curves are all similar, and again they are consistent with the calculated positive excitation energies. ${ }^{252} \mathrm{Fm}$, the $\mathrm{Fm}$ isotope requiring the transfer of the fewest number of nucleons (four protons) has the highest cross sections with $256 \mathrm{Fm}$, which requires the transfer of eight nucleons, having the lowest cross sections. $255 \mathrm{Fm}$ seems to be an exception in this case. Even though it requires the transfer of seven nucleons, it has higher cross sections at three energies than both $254 \mathrm{Fm}$ and ${ }^{250} \mathrm{Fm}$ which each require the transfer of six nucleons. The higher cross sections of $255 \mathrm{Fm}$ relative to ${ }^{254} \mathrm{Fm}$ and ${ }^{250}$ Fun cannot be explained on the basis of excitation energies. $255 \mathrm{Fm}$ has an $E_{X}$ which is $1.9 \mathrm{MeV}$ as compared to $6.2 \mathrm{MeV}$ and $2.4 \mathrm{MeV}$ for ${ }^{254} \mathrm{Fm}$ and ${ }^{250} \mathrm{Fm}$ respectively. However, it should be noted that the $255 \mathrm{Fm}$ cross sections have relatively large errors.

e. Ft and Et Values

Taile III.12 lists the calculated $F_{t}$ and $E_{t}$ values for the ${ }^{31} \mathrm{P}$ system. For 12 of the 21 cases, the value of $E_{x}$ was gre $x$ than $E_{f, n}$ resulting in a negative value for $F_{t}$. The $F_{t}{ }^{\prime}$ s from the ${ }^{31} \mathrm{P}$ system range in value from 0.01 to 0.69 , similar to the values from the ${ }^{40} \mathrm{Ar}$ and ${ }^{44} \mathrm{Ca}$ systems (discussed below). No obvious trends in the $F_{t}{ }^{\prime}$ s can be seen. There is good agreement between $\Gamma_{t}$ and $E_{t}$ for ${ }^{252} \mathrm{Cf}, 255 \mathrm{Es}, 255 \mathrm{Fm}$, and ${ }^{256} \mathrm{Fm}$. However, there are significant deviations for ${ }^{250} \mathrm{Bk},{ }^{253} \mathrm{Cf},{ }^{254} \mathrm{Es} m,{ }^{250} \mathrm{Fm}$, and $253 \mathrm{Fm}$. 


\section{F. ${ }^{40} \mathrm{Ar}-{ }^{248} \mathrm{Cm}$ System}

The cross sections obtained from the ${ }^{40} \mathrm{Ar}$ system are summarized in Table III. 4 with the statistical standard deviations, $s$.

\section{Isotopic distributions}

Isotopic distributions for $\mathrm{Bk}, \mathrm{Cf}$, Es, and Fm from the interactions of $245-\mathrm{MeV}(1.16$ $\mathrm{X}$ Coulomb barrier) ${ }^{40} \mathrm{Ar}$ ions with ${ }^{248} \mathrm{Cm}$ are plotted in Fig. III.12. The maxima of the mass-yield curves for the ${ }^{40} \mathrm{Ar}$ system occur at ${ }^{248} \mathrm{Bk}^{m},{ }^{250} \mathrm{Cf},{ }^{251} \mathrm{Es}$, and $\leq 252 \mathrm{Fm}$. Since the cross sections for the neutron-deficient Fm isotopes were not measured, the peaks of the mass distributions for the Fm isotopes for this system cannot be located with certainty. However it can be said that the peaks occur at a mass number of 252 or less. The measured isotopic distributions are essentially symmetric about the maximum with FWHM's of $3.3,2.8$, and 1.5 mass units for $\mathrm{Bk}, \mathrm{Cf}$, and $\mathrm{Es}$, respectively. In addition, the maxima and the widths of the mass-yield curves for the ${ }^{40} \mathrm{Ar}$ system stay essentially constant over the energy range in which cross sections were measured.

\section{Excitation Functions}

\section{a. Excitation functions for Bk isotopes}

Excitation functions for the Bk isotopes $244,245,246,248 m$, and 250 produced from Nie interactions of ${ }^{40} \mathrm{Ar}$ with ${ }^{248} \mathrm{Cm}$ are shown in Fig. III.13. The shapes of the functions are consistent with the calculated excitation energies. ${ }^{248} \mathrm{Bk}^{m}$ has the highest production cross section, with ${ }^{250} \mathrm{Bk}$ having the next highest, followed by $246 \mathrm{Bk}$, $245 \mathrm{Bk}$, and finally ${ }^{244} \mathrm{Bk}$.

\section{b. Excitation functions for $C f$ isotopes}

The excitation functions measured for the Cf isotopes $246,248,250,252,253$, and 254 are shown in Fig. III.14. These are all similar in shape showing an increase by a factor of 2.5 when the bombarding energy is increased by $18 \mathrm{MeV}$, then they level off for the next $41 \mathrm{MeV}$ and finally decrease by a factor of 2.5 over the next $20 \mathrm{MeV}$. The highest cross sections occur for $250 \mathrm{Cf}$ which results from the transfer of only two 
nucleons (two protons), the fewest possible, and the lowest cross sections occur for ${ }^{254} \mathrm{Cf}$ which results from the transfer of six nucleons (two protons and four neutrons).

\section{c. Excitation functions for Es isotopes}

Excitation functions for the Es isotopes 250-255 are plotted in Fig. III.15. Again the shapes of the functions are consistent with the calculated excitation energies, and again the highest cross section occurs for ${ }^{251} \mathrm{Es}$ resulting from the transfer of the fewest number of nucleons, in this case three protons. A decrease in the production cross sections is seen as the number of nucleons transferred increases.

The fraction of the yield going to ${ }^{250} \mathrm{Es} g\left(\sigma\left(6^{+}\right) /\left[\sigma\left(6^{+}\right)+\sigma\left(1^{-}\right)\right]\right)$is given in Table III.15 as a function of projectile energy for the ${ }^{40} \mathrm{Ar}$ system. These data are plotted in Fig. III.16 also. The fraction decreases with increasing projectile bombarding energy from 207 to $245 \mathrm{MeV}$, and levels off at about 0.25 over the next $41 \mathrm{MeV}$. Hence, the majority of the yield (except at the lowest bombarding energy) for this mass number is going into the low spin ${ }^{250} \mathrm{Es}^{m}\left(1^{-}\right)$. Perhaps the fraction of the yield going to ${ }^{250} \mathrm{Es} g$ is decreasing with increasing bombarding energy due to increased fission in 250 Es 8 relative to $250 \mathrm{Es}^{\mathrm{m}}$.

Chasteler et al. [CHA87], for the ${ }^{16} \mathrm{O}-{ }^{249} \mathrm{Cf}$ system, found the fraction of the yield going to ${ }^{250}$ Esg decreases linearly with increasing bombarding energy. The values went from 0.41 to 0.34 when the projectile energy was increased from 1.0 to $1.4 \mathrm{X}$ Coulomb barrier. For the ${ }^{18} \mathrm{O}-{ }^{249} \mathrm{Cf}$ system, Lee et al. [LEE83] reported the fraction going to ${ }^{250} \mathrm{Es} g$ to be $0.28,0.39,0.36$, and 0.27 at 1.0,1.1, 1.3, and $1.5 \mathrm{X}$ Coulomb barrier,

respectively. The fraction of the yield going to ${ }^{250} \mathrm{Es} g$ in the ${ }^{48} \mathrm{Ca}-{ }^{248} \mathrm{Cm}$ system 'HOF85] was reported to be 0.35 and 0.31 at 1.1 and $1.3 \times$ Coulomb barrier, respectively. Hence, it appears that in these heavy ion-actinide systems near the Coulomb barrier, the low spin isomer receives a significant fraction of the yield.

The ${ }^{254} \mathrm{Es}^{m}$ cross section seems to fit the systematics of the other Es isotopes in the mass yield curve from the ${ }^{40} \mathrm{Ar}$ systern at $1.16 \mathrm{X}$ Coulomb barrier. If a significant 
fraction of the yield was going to ${ }^{254} \mathrm{Es} g\left(7^{+}\right)$, then the cross section for ${ }^{254} \mathrm{Es}^{m}\left({ }^{+}\right)$ would appear low on the mass yield curve. Finally, since the cross sections for $248_{\mathrm{Bk}} m$ $\left(1^{-}, t_{1} / 2=23.4 \mathrm{~h}\right)$ were measured and those of $248 \mathrm{Bk} g\left(6^{+}, t_{1} / 2=>9 \mathrm{y}\right)$ were not, then one must consider the fraction of yield going to the $6^{+}$state. If one assumes that the partitioning of the yield is similar to the case of $250 \mathrm{Es}^{m}$ and $250 \mathrm{Es} g$, then the fraction of the yield going to the $6^{+}$state would be approximately $0.57,0.47,0.28,0.25$, and 0.26 at $207,225,245,266$, and $286 \mathrm{MeV}$, respectively.

\section{d. Excitation functions for Fm isotopes}

Excitation functions for isotopes of Fm are shown in Fig. III.17. The curves are all similar in shape and are consistent with their calculated positive excitation energies. The fewest number of nucleons which can be transferred to make a Fm isotope is four, and indeed, ${ }^{252} \mathrm{Fm}$ has the highest cross section. The cross sections decrease as the number of nucleons transferred increases, with ${ }^{252} \mathrm{Fm}$ having the largest cross section and 256 Fm having the smallest.

e. $F_{t}$ and $E_{t}$ Values

$F_{t}$ and $E_{t}$ values for the ${ }^{40} \mathrm{Ar}$ system are shown in Table III.13. It should be noted that the $\mathrm{Cf}$ excitation functions are relatively flat; therefore, the $E_{M}$ values for these isotopes were difficult to locate, thus affecting the $F_{t}$ values. The $F_{t}^{\prime}$ 's range in value frum 0.02 to 0.29 , with average values of $0.16,0.14,0.18$, and 0.19 for the Bk, Cf, Es, and $\mathrm{Fm}$ isotopes respectively. As can be seen, the average $F_{t}$ 's generally increase with increasing atomic number. However, $\mathrm{Cf}$ does not follow this trend. Once again, no correlation was found between $F_{t}$ and $E_{t}$ for a given nuclide; however, in general, $E_{t}$ is less than $F_{t}$ indicating that the fraction of energy transferred is greater than the fraction of mass transferred.

\section{G. ${ }^{44} \mathrm{Ca}-248 \mathrm{Cm}$ System}

Production cross sections for the measured isotopes of $\mathrm{Bk}, \mathrm{Cf}, \mathrm{Es}$, and $\mathrm{fm}$ from the interactions of ${ }^{44} \mathrm{Ca}$ with ${ }^{248} \mathrm{Cm}$ along with the statistical standard deviation, $s$, are 
shown in Table III.5.

\section{Isotopic Distributions}

Isotopic distributions for $\mathrm{Bk}, \mathrm{Cf}, \mathrm{Es}$, and $\mathrm{Fm}$ from the interactions of $27 . \mathrm{-MeV}(1.16$ $\mathrm{X}$ Coulomb barrier) ${ }^{44} \mathrm{Ca}$ ions, with ${ }^{248} \mathrm{Cm}$ are shown in Fig. III.18. The maxima of the isotopic distributims occur at $248 \mathrm{Bk},{ }^{250} \mathrm{Cf},{ }^{251} \mathrm{Es}$, and less than or equal to $252 \mathrm{Fm}$. As mentioned before, these are the nuclides which correspond to the isotopes requiring the transfer of the fewest number of nucleons for a given element (with the exception of $\left.{ }^{248} \mathrm{Bk}^{m}\right)$. Since the cross sections for the neutron-deficient fran isotopes were not measured for this system, the peak of the mass-yield curve for Fm cannot be located with certainty.

The isotopic distributions are symmetric and display full-widths-at-half-maximum of 3.(), 3.5, and 2.() for Bk, Cf, and Es respectively. The width of the Fm peak could not be determined due to insufficient data on the neutron-deficient Fm isotopes.

\section{Excitation Functions}

a. Excitation Funcrions for Bk Lonfopes

Excitation functions for the $B \mathrm{k}$ isotopes 24. 245, 246. 248m, and 250) are ploted in Fig. III.19. $248 \mathrm{Bk}$ 'm has the highest production cross sections, followed by $246 \mathrm{Bk}$, 25()$_{\mathrm{Bk}}, 245 \mathrm{Bk}$ and fimally $244 \mathrm{Bk}$. The production of $248 \mathrm{Bk}$ requires the transfer of one proton and one neutron as does the production of ${ }^{25()} \mathrm{Bk}$. However, the $1.4 \mathrm{MeV} E_{X}$ value of 248 BK makes the production of this nuclide more energetically favorable than the production of 25$)^{3 \mathrm{~B}}$ which has an $E_{\mathrm{x}}$ value of $-4.7 \mathrm{MeV}$. $246_{\mathrm{Bk}}$, which requires the transfer of 4 nucleons, has cross sections which are greater at four energes than the cross sections of ${ }^{25()} \mathrm{Bk}$. This again can be attributed to the positive $E_{x}$ value $(5.5 \mathrm{MeV}$ ) for this reaction channel as opposed to the negative value for the ${ }^{250} \mathrm{Bk}$ channel. The shape of $250 \mathrm{Bk}$ 's excitation function is consistent with its negative $E_{x}$ value of $-4.7 \mathrm{MeV}$. However, the excitation function shapes of the other Bk isotopes are inconsistent with their calculated positive $E_{\mathcal{X}}$ values. 


\section{b. Excitation Functions for $C f$ Isotopes}

Excitation functions for the Cf isotopes 246, 247, 248, 250, 252, and 253 are plotted in Fig. III.20. The isotope requiring the transfer of the fewest nucleons, ${ }^{250} \mathrm{Cf}$, (two protons) has the highest production cross sections at all of the measured energies. ${ }^{248} \mathrm{Cf}$ has the next highest cross sections followed by ${ }^{252} \mathrm{Cf},{ }^{247} \mathrm{Cf},{ }^{246} \mathrm{Cf}$, and finally ${ }^{253} \mathrm{Cf}$. Both ${ }^{248} \mathrm{Cf}$ and ${ }^{252} \mathrm{Cf}$ require the transfer of four nucleons; however, ${ }^{248} \mathrm{Cf}$ has a positive $E_{X}$ value $(7.2 \mathrm{MeV})$ whereas $252 \mathrm{Cf}$ has a negative value $(-0.2 \mathrm{MeV})$. Five nucleons have to be transferred between the target and projectile in order to produce both ${ }^{247} \mathrm{Cf}$ and ${ }^{253} \mathrm{Cf}$. ${ }^{247} \mathrm{Cf}$ has much higher cross sections than $253 \mathrm{Cf}$, as expected from their calculated $E_{X}$ values of $5.9 \mathrm{MeV}$ and $-5.7 \mathrm{MeV}$ respectively. ${ }^{246} \mathrm{Cf},{ }^{247} \mathrm{Cf}$, and ${ }^{250} \mathrm{Cf}$ have excitation functions which are consistent with their calculated positive $E_{X}$ values. Additionally the shapes of the excitation functions from ${ }^{252} \mathrm{Cf}$ and ${ }^{253} \mathrm{Cf}$ are consistent with their calculated negative $E_{X}$ values.

\section{c. Excitation Functions for Es Isotopes}

Excitation functions of the Es isotopes 250, 251,252, 253, and 254m are shown in Fig. III.21. The solid lined labeled 25() is the sum of the cross sections from $250 \mathrm{~m}$ and $250 \mathrm{~g}$. ${ }^{251} \mathrm{Es}$, the isotope requiring the transfer of the fewest nucleons, has the highest peak cross section and the highest cross sections at three energies. ${ }^{250}$ Es has the next highest cross sections, followed by $252 \mathrm{Es},{ }^{253} \mathrm{Es}$, and ${ }^{254} \mathrm{Es}^{m}$. Hence, as the number of nucleons transferred increases, the cross sections decrease. ${ }^{252} \mathrm{Es},{ }^{253} \mathrm{Es}$, and ${ }^{254} \mathrm{Es}^{m}$ have excitation functions which are consistent with their negative $E_{x}$ values. Both ${ }^{251}$ Es and ${ }^{250} \mathrm{E}$ Es, however, have excitation functions which are inconsistent with their calculated positive excitation energies. But if one looks at the excitation functions of ${ }^{250} \mathrm{Es}^{g}$ and ${ }^{250} \mathrm{Es}^{m}$ separately, it appears that ${ }^{250} \mathrm{Es} g$ has an excitation function that is consistent with a positive $E_{X}$ while ${ }^{250}$ Es $^{m}$ has one that is consistent with a negative $E_{X}$. ${ }^{250} \mathrm{Es}^{m}$ has its peak cross section at $275 \mathrm{MeV}$ while the peak cross section of ${ }^{250} \mathrm{Es} g$ is at $252 \mathrm{MeV}$. Table III.15 lists the fraction of the yield going to $250 \mathrm{Es} g\left(6^{+}\right)$as a 
function of projectile bombarding energy for the ${ }^{44} \mathrm{Ca}$ system. These data are also plotted in Fig. III.22. Like the ${ }^{40} \mathrm{Ar}$ system, the fraction decreases with increasing projectile energy up to $275 \mathrm{MeV}$, and then stays relatively constant over the next 44 $\mathrm{MeV}$. Based upon these results, the fraction of the yield going to $248 \mathrm{Bkg}\left(6^{+}\right)$can be estimated to be $0.38,0.29,0.15,0.19$, and $>0.16$ at $232,252,275,297$, and $319 \mathrm{MeV}$, respectively. The ${ }^{254} \mathrm{Es}^{\mathrm{m}}$ cross section does not appear to be unusually low on the mass yield curve when compared to the other Es isotopes from the ${ }^{44} \mathrm{Ca}$ system. Hence, it appears only a small fraction of the yield for mass number 254 is going to ${ }^{254}$ Esg $\left(7^{+}\right)$.

\section{d. Excitation Functions for Fm lsotopes}

Fig. III.23 displays excitation functions for the Fm isotopes 252, 253, 254, and 256. The isotope requiring the transfer of the fewest number of nucleons, $252 \mathrm{Fm}$ (four nucleons) has the highest cross sections, followed by $25.3 \mathrm{Fm}$ (five nucleons), ${ }^{254} \mathrm{Fm}$ (six nucleons), and $256 \mathrm{Fm}$ (eight nucleons). The shapes of these excitation functions are all consistent with their calculated positive $E_{x}$ values.

e. Find E, lalues

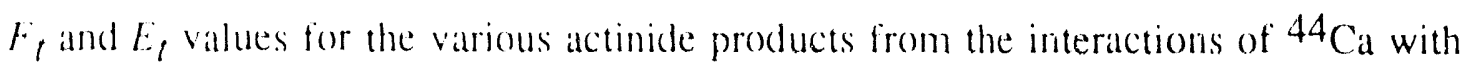

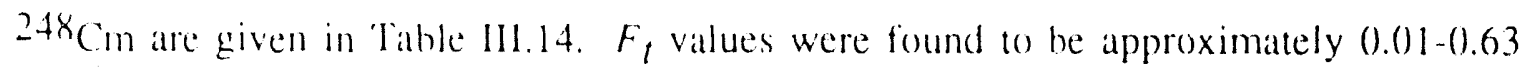
with average values of $\leq(10.03,0.22,0.24$, and 0.14 for the Bk, Cf, Es, and Fm isotopes respectively. The average $F$ values increase with increasing $Z$ going from Bk to $\mathrm{Fm}$ with the exception of $F$ muclide. As with the ${ }^{12} \mathrm{C}$ and ${ }^{40} \mathrm{Ar}$ systems, $E_{t}$ is less than $F_{t}$ indicating that the fraction of energy transferred is greater than the fraction of mass transterred. Lee et al. [LEE83] also found this to be the case for the ${ }^{18} \mathrm{O}-248 \mathrm{Cm}$ and ${ }^{18} \mathrm{O}-249 \mathrm{Cf}$ systems, where the avelage $F_{t}$ value was approximately 0.5 . The calculated $F_{t}$ values for the ${ }^{44} \mathrm{Ca}$ system would be smaller than those from the ${ }^{18} \mathrm{O}-248 \mathrm{Cm}$ and ${ }^{18} \mathrm{O}-249 \mathrm{Cf}$ systems if the energy transferred is proportional to the fraction of mass transferred. 


\section{H. Comparison of the Various Heavy Ion- $248 \mathrm{Cm}$ Systems}

The maxima of the isotopic distributions for the ${ }^{12} \mathrm{C},{ }^{18} \mathrm{O},{ }^{31} \mathrm{P},{ }^{40} \mathrm{Ar},{ }^{40} \mathrm{Ca},{ }^{44} \mathrm{Ca}$, ${ }^{48} \mathrm{Ca}$, and ${ }^{132} \mathrm{Xe}$ systems are listed in Table III.16 [HOF82] [LEY90] [LEY90A] [TÜR90], along with the neutron-to-proton ratios of the projectiles. All of the systems show maximum cross sections for Bk at mass 248 except for the most neutron-deficient projectile, ${ }^{40} \mathrm{Ca}$, for which the maximum occurs at mass 247 , and the neutron rich projectiles $18 \mathrm{O},{ }^{48} \mathrm{Ca}$, and $132 \mathrm{Xe}$ which have maxima at about mass number 249 . The maximum cross sections for $C f$ occur at mass 250 in all cases except for the ${ }^{40} \mathrm{Ca}$ system again, for which the maximum is at 248. The maxima of the Es curves are at mass 251 for ${ }^{12} \mathrm{C},{ }^{31} \mathrm{P},{ }^{44} \mathrm{Ca},{ }^{40} \mathrm{Ar}$, and ${ }^{1.32} \mathrm{Xe}$, at 250 for ${ }^{40} \mathrm{Ca}, \leq 2.52$ for ${ }^{18} \mathrm{O}$, and at 2.52 for ${ }^{48} \mathrm{Ca}$. The Fm curves have their maxima around 252 for the ${ }^{40} \mathrm{Ca},{ }^{31} \mathrm{P},{ }^{44} \mathrm{Ca}$, and ${ }^{40} \mathrm{Ar}$ systems, at 253 for the ${ }^{12} \mathrm{C}$ and $132 \mathrm{Xe}$ systems, and around 254 for the $18 \mathrm{O}$ and ${ }^{48} \mathrm{Ca}$ systems. Once again, it should be noted that the maximum cross sections for the $4\left({ }^{\circ} \mathrm{Ar}\right.$ and ${ }^{44} \mathrm{Ca}$ systems could not be located with certainty due to insufficient data on the neutron-deficient Fm isotopes.

The values of the FWHM's for the isotopic distributions from the ${ }^{12} \mathrm{C},{ }^{4}{ }^{(} \mathrm{Ca},{ }^{31} \mathrm{P}$, ${ }^{44} \mathrm{Ca},{ }^{40} \mathrm{Ar},{ }^{18} \mathrm{O},{ }^{48} \mathrm{Ca}$, and ${ }^{132} \mathrm{Xe}$ systems are shown in Table III.17. The ${ }^{12} \mathrm{C}$ system has the smallest FWHM's and 132Xe has the largest. However, there doun't seem to be any pattern in the FWHM's from the other systems. Additionally, the FWHM values for these system are similar to the value of 3.0 reported previously for the $238 \mathrm{U}$ system [SCH82].

The final isotopic distributions for Bk from the interactions of a number of heavy ions (HI) with ${ }^{248} \mathrm{Cm}$ are shown in Fig. III.24. The $\Delta N$ on the $\mathrm{x}$ axis represents the total number of neutrous transfered. A ( + ) sign indicates neutrons are transferred from the projectile to the target, while a (-) sign indicates the opposite. The ${ }^{31} \mathrm{P}$ system has the highest production cross section for ${ }^{248} \mathrm{Bk} m$ by about a factor of two. This high peak cross section for ${ }^{248} \mathrm{Bk}^{m}$ might be due to the odd proton in ${ }^{31} \mathrm{P}$. Since an odd proton 
would be unpaired in the nucleus, the probability of transferring one unpaired proton from an odd $Z$ nucleus to the target should be greater than the probability of transferring one proton from an even $Z$ nucleus. This argument is supported by the $E_{X}$ values for Bk isotopes from these systems (Table III.6). The $E_{x}$ values from the ${ }^{31} \mathrm{P}$ system are greater than the corresponding values from ${ }^{12} \mathrm{C},{ }^{40} \mathrm{Ar},{ }^{44} \mathrm{Ca}$, and ${ }^{48} \mathrm{Ca}$ systems. However, for some neutron-deficient $\mathrm{Bk}$ isotopes $(244-247 \mathrm{Bk})$, the ${ }^{40} \mathrm{Ca}$ system has $E_{X}$ values that are greater than those of the ${ }^{31} \mathrm{P}$ system. This should be expected since the neutron-toproton ratio of ${ }^{40} \mathrm{Ca}$ is only 1.00 . With such a low neutron-to-proton ratio, ${ }^{40} \mathrm{Ca}$ should preferentially transfer protons to the target nucleus. ${ }^{12} \mathrm{C}$ has the lowest cross sections for all of the Bk isotopes measured. This is to be expected, since the $E_{X}$ values for this system are generally the most negative of the six systems listed. The neutron-deficient Bk isotope yields are essentially independent of the projectile (with the exception of the neutron-rich ${ }^{48} \mathrm{Ca}$ curve and the energetically unfavorable ${ }^{12} \mathrm{C}$ curve), whereas the neutron-rich $\mathrm{Bk}$ isotopes are projectile dependent. ${ }^{48} \mathrm{Ca}$, the most neutron-rich projectile gives the highest yields while ${ }^{12} \mathrm{C}$ and ${ }^{4(} \mathrm{Ca}$, the most neutron-deficient projectiles, give the lowest yields of the neutron-rich Bk isotopes.

Isotopic distributions for $\mathrm{Cf}$ from the reactions of several heavy ions with ${ }^{248} \mathrm{Cm}$ are shown in Fig. III.25. The energetically unfavorable ${ }^{12} \mathrm{C}$ and ${ }^{48} \mathrm{Ca}$ systems (see Table III.7) give the lowest cross sections for the neutron-deficient $\mathrm{Cf}$ isotopes. The cross sections for the neutron-rich $\mathrm{Cf}$ isotopes are all quite similar for ${ }^{31} \mathrm{P},{ }^{40} \mathrm{Ar},{ }^{44} \mathrm{Ca}$, and ${ }^{48} \mathrm{Ca}$. The most neutr deficient projectiles, ${ }^{12} \mathrm{C}$ and ${ }^{40} \mathrm{Ca}$, are the exceptions, with the lowest cross sections tor the neutron-rich Cf isotopes.

Isotopic distributions for Es produced from the interactions of some heavy ions :vith ${ }^{248} \mathrm{Cm}$ are shown in Fig III.26. ${ }^{40} \mathrm{Ar}$ consistently has the highest yields for the isotopes 250-254Es. The curves for the ${ }^{12} \mathrm{C}$ and ${ }^{31} \mathrm{P}$ sysims seem to have the narrowest FWHM's.

Fig. III.27 illustrates the final isotopic distributions for Fm produced from the 
interactions of several heavy ions with ${ }^{248} \mathrm{Cm}$. ${ }^{40} \mathrm{Ar}$ has the highest cross sections for the neutron-rich $\mathrm{Fm}$ isotopes, and ${ }^{48} \mathrm{Ca}$ has the lowest cross sections for the neutrondeficient Fm isotopes.

Based upon the detailed comparison of the interactions of numerous heavy ions with ${ }^{248} \mathrm{Cm}$, the following observations can be made:

1. In general the cross sections decrease as the number of nucleons transferred increases.

2. With few exceptions, the shapes of the excitation functions are consistent with the calculated excitation energies. Specifically, for reactions with negative excitation energies, the cross sections increase with increasing projectile bombarding energy until the reaction barrier is exceeded. Once the maximum is reached at some energy above the Coulomb barrier, the cross sections decrease due to fission and/or particle emission. Reactions with positive excitation energies display excitation functions that peak at or near the Coulomb barrier and decrease with increasing projectile kinetic energy.

3. The maxima of the isotopic distributions occur for those reaction channels which involve the apparent transfer of the fewest number of nucleons for which $E_{X}$ is positive. Tt: lends support to a binary-type reaction mechanism in which the target-like fragment has little excitation energy. However, deviations from this behavior, such as, the ${ }^{40} \mathrm{Ca}$ system indicate that factors other than $E_{X}$ might also be influencing the final production cross section distributions.

4. The shapes of the isotopic distributions are all fairly symmetric about the maximum, with a FWHM of $\equiv 2.5$ mass units. The symmetric isotopic distributions should follow for a given $Z$ if the production cross section for transferring $+n$ neutrons (transferring neutrons from the projectile to the target) is equal to the production cross section for transferring - $n$ neutrons provided the $E_{X}$ values for the two reaction channels are similar.

5. The neutron-rich projectiles such as ${ }^{48} \mathrm{Ca}$ enhance the production of neutron-rich 
products, while neutron-deficient projectiles such as ${ }^{12} \mathrm{C}$ and ${ }^{40} \mathrm{Ca}$ enhance the production of neutron-deficient products. 


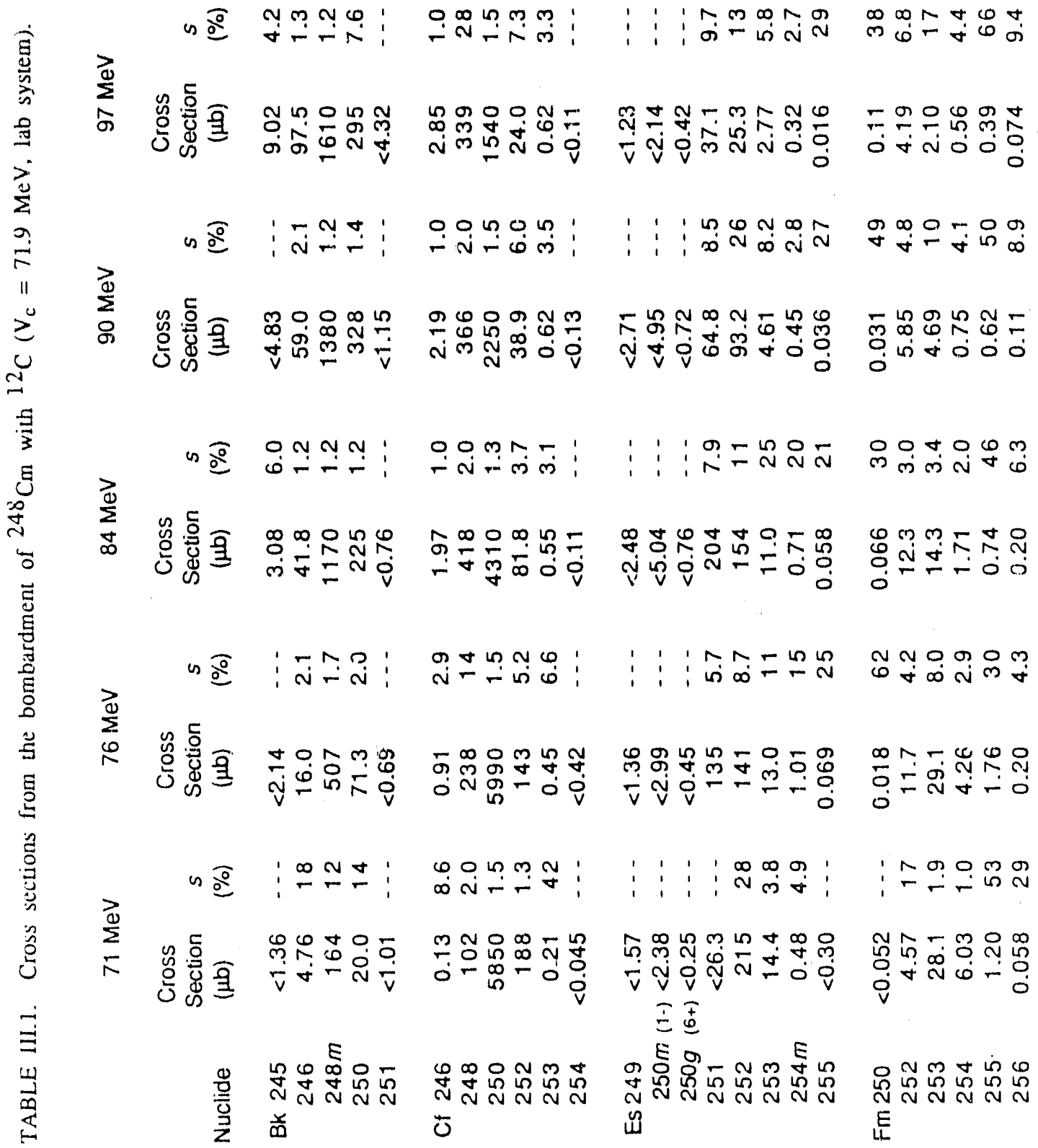


Table III.2. Cross sections for Md and No isotopes produced from the bombardment of ${ }^{248} \mathrm{Cm}$ with ${ }^{12} \mathrm{C}$ ions.

\begin{tabular}{|c|c|c|c|c|c|c|}
\hline Nuclide & $\begin{array}{l}\quad 71 \mathrm{M} \\
\text { Cross } \\
\text { section } \\
(\mu \mathrm{b})\end{array}$ & $\begin{array}{l}\mathrm{leV} \\
s \\
(\%)\end{array}$ & $\begin{array}{r}76 \\
\text { Cross } \\
\text { section } \\
(\mu b)\end{array}$ & $\begin{array}{l}\text { leV } \\
s \\
(\%)\end{array}$ & $\begin{array}{r}84 \\
\text { Cross } \\
\text { section } \\
(\mu b)\end{array}$ & $\begin{array}{l}s \\
(\%)\end{array}$ \\
\hline Md 254 & 0.053 & 26 & $\ldots$ & -- & $\ldots$ &.- \\
\hline 255 & 0.33 & 11 & $-\cdots$ & -- & ..... & -. \\
\hline 256 & 0.049 & 13 & 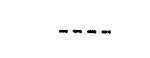 & -- & -..- & -- \\
\hline 257 & $<0.048$ & -- & $\ldots$ & -- & $\ldots$ & -- \\
\hline No 254 & -... & -. & $-\ldots$ & - & 0.050 & 14 \\
\hline 255 & 0.022 & 17 & 0.177 & 4 & 0.077 & 8 \\
\hline 256 & 0.203 & 10 & 0.075 & 15 & 0.024 & 56 \\
\hline 257 & 0.033 & 11 & 0.015 & 19 & 0.010 & 45 \\
\hline
\end{tabular}




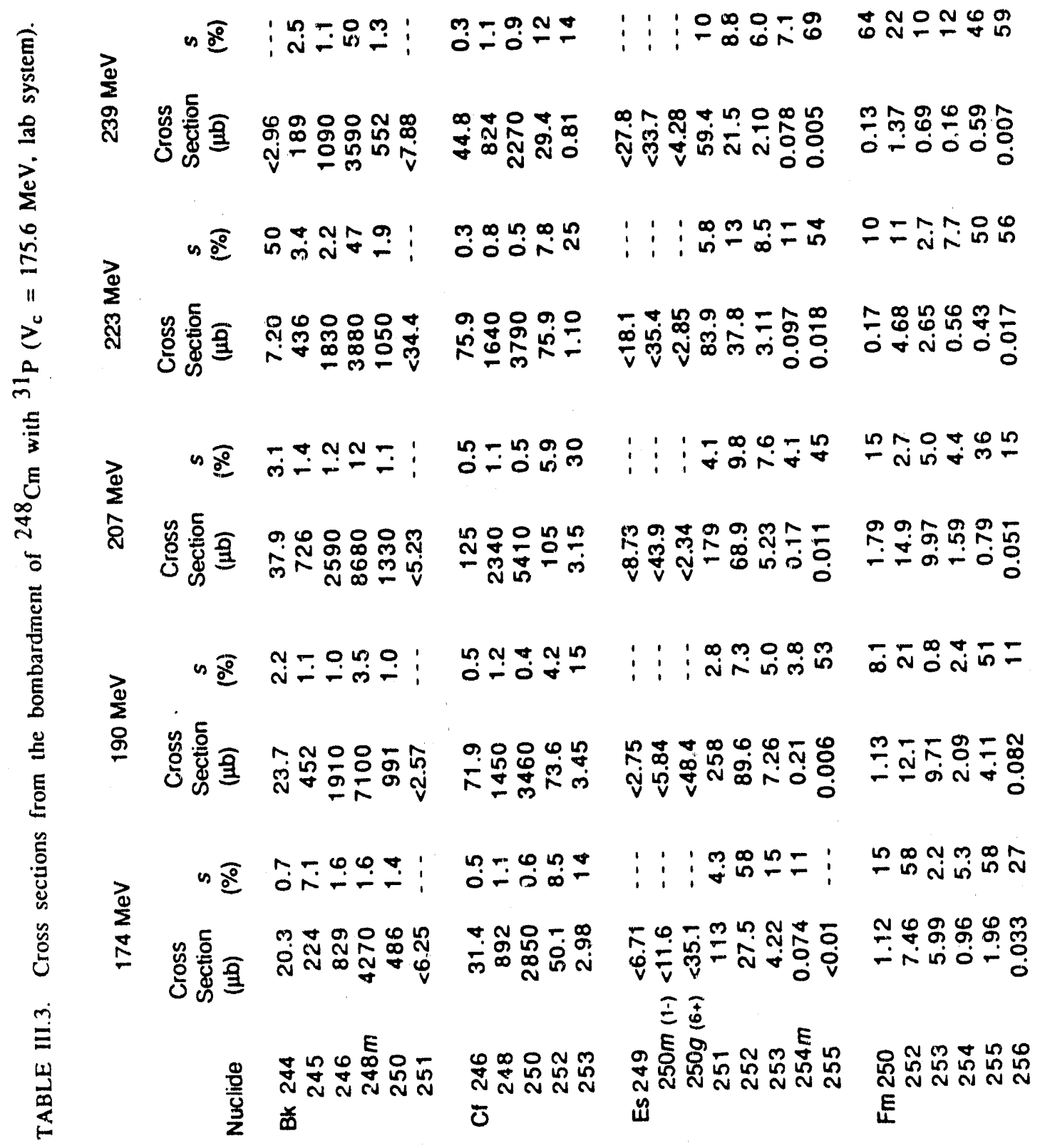




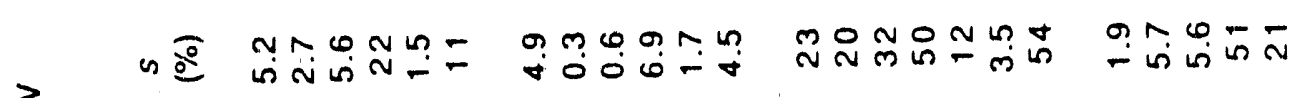

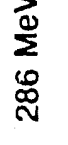
号 兽

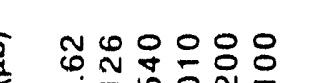

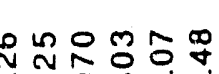

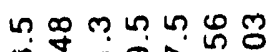
$\stackrel{\infty}{\sim} \stackrel{\sim}{\sim} \underset{v}{\sim}$

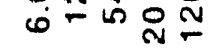
ले बूल ले $\stackrel{\infty}{\infty}$ 元 NNOOO

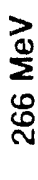

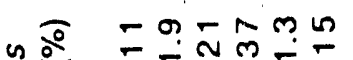

m n n n n n

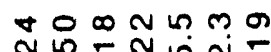

NON MN

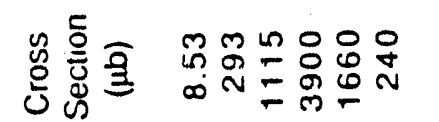

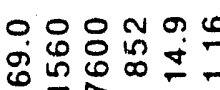

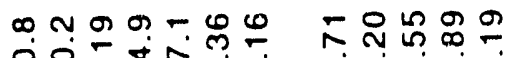

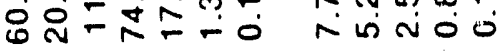

a $9 \sin -\pi n$

n

$n=\cos =m-\infty$

- 0 or us 0

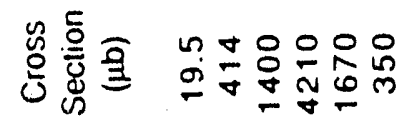

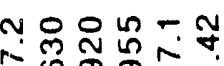

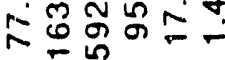

ம0

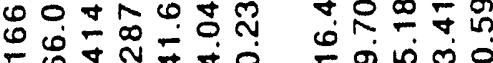
$n-\infty$

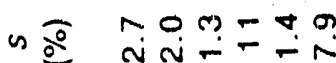

$\forall \ln \sim \pi \sigma$

n

$0 \infty \forall \%$

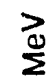

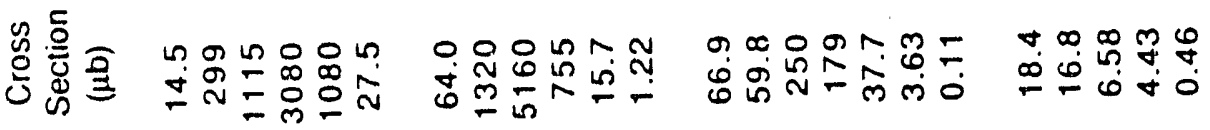

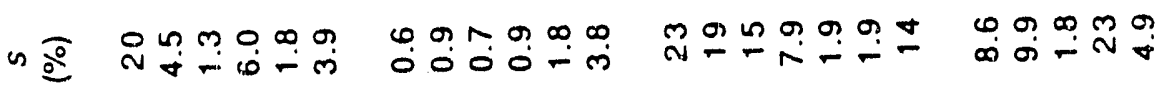

$\stackrel{2}{2}$

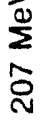

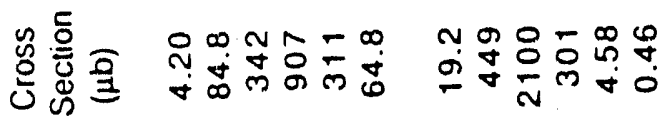

man

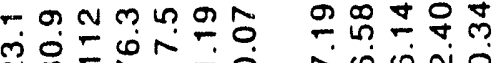
$\pm \underset{ \pm}{ \pm}$

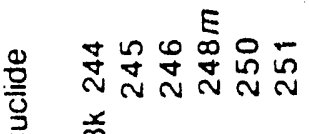
离

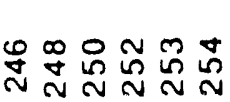

$\tilde{\omega}$

E $\bar{O}-\sim m$ E

ก

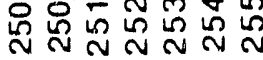
แี

กิ้

E 


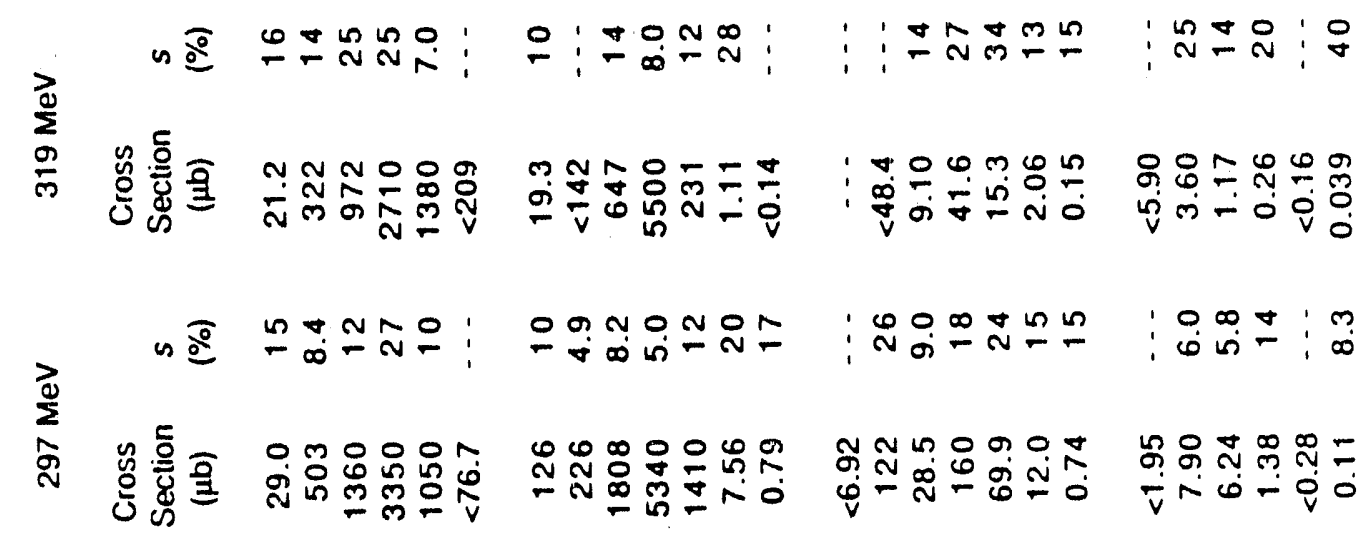

ব) य

ลิ

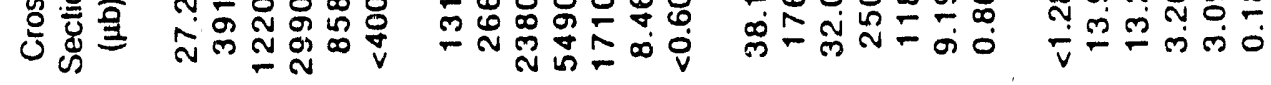

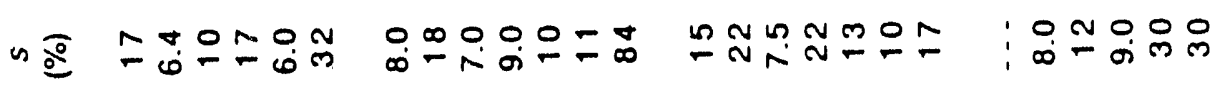

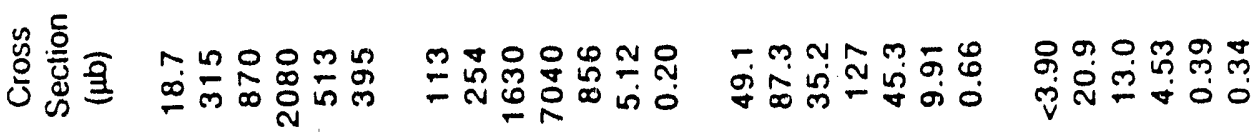
य

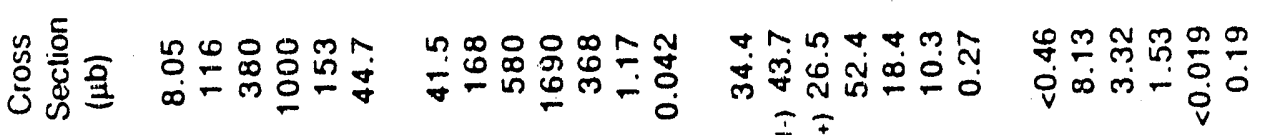

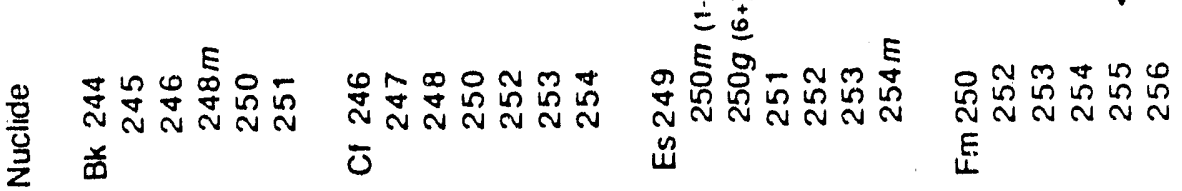


Table III.6. Excitation energies, $E_{x}$, for $\mathrm{Bk}$ isotopes produced from the interactions of various heavy ions with $248 \mathrm{Cm}$.

\begin{tabular}{|c|c|c|c|c|c|c|}
\hline $\begin{array}{l}\text { Product } \\
\text { Nuclide }\end{array}$ & ${ }^{12} \mathrm{C}$ & $31 \mathrm{P}$ & ${ }^{40} \mathrm{Ar}$ & ${ }^{40} \mathrm{Ca}$ & ${ }^{44} \mathrm{Ca}$ & ${ }^{48} \mathrm{Ca}$ \\
\hline Bk 244 & -19.2 & 7.6 & 0.1 & 17.2 & 6.8 & -16.4 \\
\hline 245 & -12.2 & 11.3 & 3.0 & 16.5 & 8.8 & -10.9 \\
\hline 246 & -9.3 & 8.6 & 1.3 & 11.9 & 5.5 & -9.6 \\
\hline 247 & -3.5 & 10.8 & 2.9 & 11.1 & 5.4 & -4.4 \\
\hline 248 & -3.5 & 6.4 & -0.2 & 5.8 & 1.4 & -5.0 \\
\hline 249 & -0.7 & 6.0 & 0.2 & 4.2 & 0.3 & -3.4 \\
\hline 250 & -7.6 & 0.0 & -3.2 & -4.3 & -4.7 & -7.1 \\
\hline 251 & -9.9 & -2.3 & -3.2 & -10.2 & -6.0 & -7.7 \\
\hline
\end{tabular}


Table III.7. Excitation energies, $E_{x}$, for $\mathrm{Cf}$ isotopes produced from the interactions of various heavy ions with $248 \mathrm{Cm}$.

\begin{tabular}{rrrrrrr}
\hline & & & & & & \\
\hline Product & & & & & & \\
Nuclide & $12 \mathrm{C}$ & $31 \mathrm{P}$ & $40 \mathrm{Ar}$ & $40 \mathrm{Ca}$ & $44 \mathrm{Ca}$ & $48 \mathrm{Ca}$ \\
\hline & & & & & & \\
\hline
\end{tabular}


Table III.8. Excitation energies, $E_{X}$, for Es isotopes produced from the interactions of various heavy ions with ${ }^{248} \mathrm{Cm}$.

\begin{tabular}{|c|c|c|c|c|c|c|}
\hline $\begin{array}{l}\text { Product } \\
\text { Nuclide }\end{array}$ & ${ }^{12} \mathrm{C}$ & $31 \mathrm{P}$ & ${ }^{40} \mathrm{Ar}$ & ${ }^{40} \mathrm{Ca}$ & ${ }^{44} \mathrm{Ca}$ & ${ }^{48} \mathrm{Ca}$ \\
\hline Es 249 & -11.7 & 8.6 & -1.3 & 15.8 & 2.3 & -16.8 \\
\hline 250 & -6.9 & 7.1 & -1.4 & 13.4 & 1.2 & -14.8 \\
\hline 251 & 0.4 & 9.7 & 1.4 & 13.7 & 2.5 & -10.6 \\
\hline 252 & 1.3 & 6.1 & 0.0 & 8.3 & -0.5 & -12.2 \\
\hline 253 & 5.3 & 5.6 & 2.1 & 5.6 & -0.3 & -9.8 \\
\hline 254 & 2.7 & -0.8 & -1.7 & -2.3 & -3.7 & -11.8 \\
\hline 255 & 2.7 & -2.5 & -2.3 & -8.2 & -4.1 & -11.2 \\
\hline 256 & -14.4 & -14.5 & -7.8 & -19.4 & -9.8 & -14.5 \\
\hline
\end{tabular}


Table 1II.9. Excitation energies, $E_{x}$, for Fm isotopes produced from the interactions of various heavy ions with ${ }^{248} \mathrm{Cm}$.

\begin{tabular}{|c|c|c|c|c|c|c|}
\hline $\begin{array}{l}\text { Product } \\
\text { Nuclide }\end{array}$ & ${ }^{12} \mathrm{C}$ & ${ }^{31} \mathrm{P}$ & ${ }^{40} \mathrm{Ar}$ & ${ }^{40} \mathrm{Ca}$ & ${ }^{44} \mathrm{Ca}$ & ${ }^{48} \mathrm{Ca}$ \\
\hline $\mathrm{Fm} 251$ & -5.2 & 4.0 & -3.1 & 16.1 & 0.4 & -18.7 \\
\hline 252 & 3.0 & 7.2 & 1.4 & 18.7 & 3.4 & -13.1 \\
\hline 253 & 5.7 & 5.6 & 0.9 & 13.9 & 1.3 & -14.4 \\
\hline 254 & 12.5 & 6.2 & 4.2 & 13.1 & 3.2 & 10.5 \\
\hline 255 & 15.5 & 1.9 & 1.2 & 6.5 & 0.0 & -11.6 \\
\hline 256 & 22.4 & 0.9 & 2.7 & 3.8 & 1.7 & -9.5 \\
\hline
\end{tabular}


Table III.10. Excitation energies, $E_{x}$, for Md isotopes produced from the interactions of various heavy ions with ${ }^{248} \mathrm{Cm}$.

\begin{tabular}{ccrrrrr}
\hline Product & & & & & & \\
\hline Nuclide & $12 \mathrm{C}$ & $31 \mathrm{P}$ & $40 \mathrm{Ar}$ & $40 \mathrm{Ca}$ & $44 \mathrm{Ca}$ & $48 \mathrm{Ca}$ \\
\hline & & & & & & \\
\hline
\end{tabular}


Table III.11. Calculated fraction of energy transferred, $F_{t}$, from the ${ }^{12} \mathrm{C}$ projectile to the ${ }^{248} \mathrm{Cm}$ target. $E_{M}$ is the maximum in the experimental excitation functions and $E_{t}$ is the fraction of energy transferred assuming energy transfer is proportional to mass transfer. $V_{c} \cong 72 \mathrm{MeV}$ in the lab frame. An * indicates that $\left(E_{f, n}-E_{x}\right)<0$. See explanation in text. Data taken from [LEY90A].

\begin{tabular}{|c|c|c|c|c|c|c|c|}
\hline Nuclide & $\begin{array}{c}E_{M} \\
(\mathrm{MeV})\end{array}$ & $F_{t}$ & $E_{t}$ & Nuclide & $\begin{array}{c}E_{M} \\
(\mathrm{MeV})\end{array}$ & $F_{t}$ & $E_{t}$ \\
\hline Bk 245 & $>97$ & $<0.75$ & 0.42 & Es 251 & 81 & 0.55 & 0.25 \\
\hline 246 & $>97$ & $<0.62$ & 0.33 & 252 & 72 & $\cdots$ & 0.33 \\
\hline $248 m$ & $>97$ & $<0.38$ & 0.17 & 253 & 72 & $\cdots$ & 0.42 \\
\hline \multirow[t]{2}{*}{250} & 90 & 0.72 & 0.17 & $254 m$ & 76 & 0.66 & 0.50 \\
\hline & & & & 255 & 76 & 0.67 & 0.58 \\
\hline Cf 246 & $>97$ & $<0.82$ & 0.50 & Fm 252 & 80 & 0.34 & 0.33 \\
\hline 248 & 84 & 0.68 & 0.33 & 253 & 76 & $*$ & 0.42 \\
\hline 250 & 76 & 0.08 & 0.17 & 254 & 72 & $*$ & 0.50 \\
\hline 252 & 72 & $*$ & 0.33 & 255 & 76 & $*$ & 0.58 \\
\hline 253 & $>90$ & $<0.71$ & 0.42 & 256 & 80 & $*$ & 0.67 \\
\hline
\end{tabular}


Table III.12. Calculated fraction of energy transferred, $F_{t}$, from the ${ }^{31} \mathrm{P}$ projectile to the ${ }^{248} \mathrm{Cm}$ target. $E_{M}$ is the maximum in the experimental excitation functions and $E_{t}$ is the fraction of energy transferred assuming energy transfer is proportional to mass transfer. $V_{C} \cong 176 \mathrm{MeV}$ in the lab frame. An * indicates that $\left(E_{f, n}-E_{x}\right)<0$. See explanation in text. Taken from [LEY90A].

\begin{tabular}{|c|c|c|c|c|c|c|c|}
\hline Nuclide & $\begin{array}{c}E_{M} \\
(\mathrm{MeV})\end{array}$ & $F_{t}$ & $E_{t}$ & Nuclidie & $\begin{array}{c}E_{M} \\
(\mathrm{MeV})\end{array}$ & $F_{t}$ & $E_{t}$ \\
\hline Bk 244 & 200 & $*$ & 0.19 & Es 251 & 195 & * & 0.10 \\
\hline 245 & 207 & $*$ & 0.16 & 252 & 195 & $*$ & 0.13 \\
\hline 246 & 207 & $*$ & 0.13 & 253 & 190 & * & 0.16 \\
\hline $248 m$ & 200 & $*$ & 0.06 & $254 m$ & 195 & 0.32 & 0.19 \\
\hline 250 & 207 & 0.17 & 0.06 & 255 & 220 & 0.18 & 0.23 \\
\hline Cf 246 & 207 & $*$ & 0.19 & $\mathrm{Fm} 250$ & 202 & 0.13 & 0.19 \\
\hline 248 & 207 & $*$ & 0.13 & 252 & 201 & $*$ & 0.13 \\
\hline 250 & 207 & $*$ & 0.06 & 253 & 200 & 0.01 & 0.16 \\
\hline 252 & 207 & 0.15 & 0.13 & 254 & 196 & $*$ & 0.19 \\
\hline \multirow[t]{2}{*}{253} & 195 & 0.69 & 0.16 & 255 & 190 & 0.26 & 0.23 \\
\hline & & & & 256 & 195 & 0.25 & 0.26 \\
\hline
\end{tabular}


TABLE III.13. Calculated fraction of energy transferred, $F_{t}$, from ${ }^{40} \mathrm{Ar}$ projectile to ${ }^{248} \mathrm{Cm}$ target. $E_{M}$ is the maximum in the experimental excitation functions, and $E_{t}$ is the fraction of energy transferred assuming it is apportioned according to the mass fraction transferred. $V_{c} \cong 211 \mathrm{MeV}$ in the lab frame. Data taken from [LEY90].

\begin{tabular}{cccccccc}
\hline & & & & & & & \\
\hline Nuclide & $E_{M}$ & $F_{t}$ & $E_{t}$ & Nuclide & $E_{M}$ & $F_{t}$ & $E_{t}$ \\
& $(\mathrm{MeV})$ & & & & $(\mathrm{MeV})$ & & \\
\hline Bk 244 & 240 & 0.21 & 0.15 & Es 250 & 243 & 0.21 & 0.10 \\
245 & 245 & 0.10 & 0.13 & 251 & 245 & 0.12 & 0.08 \\
246 & 242 & 0.15 & 0.10 & 252 & 240 & 0.18 & 0.10 \\
$248 m$ & 250 & 0.15 & 0.05 & 253 & 237 & 0.13 & 0.13 \\
250 & 255 & 0.19 & 0.05 & $254 m$ & 237 & 0.26 & 0.15 \\
& & & & 255 & 250 & 0.20 & 0.18 \\
Cf 246 & 245 & 0.10 & 0.15 & Fm 252 & 235 & 0.18 & 0.10 \\
248 & 255 & 0.02 & 0.10 & 253 & 228 & 0.28 & 0.13 \\
250 & 266 & 0.02 & 0.05 & 254 & 220 & 0.17 & 0.15 \\
252 & 245 & 0.08 & 0.10 & 255 & 230 & 0.21 & 0.18 \\
253 & 245 & 0.23 & 0.13 & 256 & 245 & 0.09 & 0.20 \\
254 & 245 & 0.29 & 0.15 & & & & \\
\hline
\end{tabular}


TABLE III.14. Calculated fraction of energy transferred, $F_{t}$, from ${ }^{44} \mathrm{Ca}$ projectile to ${ }^{248} \mathrm{Cm}$ target. $E_{M}$ is the maximum in the experimental excitation functions, and $E_{t}$ is the fraction of energy transferred assuming it is apportioned according to the mass fraction transferred. $V_{C} \cong 237 \mathrm{MeV}$ in the lab frame. An * indicates that $\left(E_{f, n}-E_{X}\right)<0$. See explanation in text. Data taken from [LEY90].

\begin{tabular}{cccccccc}
\hline & & & & & & & \\
\hline Nuclide & $E_{M}$ & $F_{t}$ & $E_{t}$ & Nuclide & $E_{M}$ & $F_{t}$ & $E_{t}$ \\
& $(\mathrm{MeV})$ & & & & $(\mathrm{MeV})$ & & \\
\hline Bk 244 & 285 & $*$ & 0.14 & Es 250 & 275 & 0.11 & 0.09 \\
245 & 285 & $*$ & 0.11 & 251 & 275 & 0.08 & 0.07 \\
246 & 282 & 0.01 & 0.09 & 252 & 273 & 0.16 & 0.09 \\
$248 m$ & 286 & 0.08 & 0.05 & 253 & 266 & 0.20 & 0.11 \\
250 & $\geq 319$ & $\leq 0.01$ & 0.05 & $254 m$ & 273 & 0.24 & 0.14 \\
& & & & 255 & 252 & 0.63 & 0.16 \\
Cf 246 & 275 & $*$ & 0.14 & Fm 252 & 252 & 0.16 & 0.09 \\
247 & 275 & $*$ & 0.11 & 253 & 264 & 0.16 & 0.11 \\
248 & 275 & $*$ & 0.09 & 254 & 256 & 0.13 & 0.14 \\
250 & 260 & 0.21 & 0.05 & 256 & 252 & 0.26 & 0.18 \\
252 & 280 & 0.13 & 0.09 & & & & \\
253 & 270 & 0.32 & 0.11 & & & & \\
254 & 292 & 0.21 & 0.14 & & & & \\
\hline
\end{tabular}


Table III.15. Fraction of yivid going to ${ }^{250} \mathrm{Es} g\left(6^{+}\right)$from the ${ }^{40} \mathrm{Ar}$ and ${ }^{44} \mathrm{Ca}$ systems.

\begin{tabular}{|c|c|c|c|c|c|}
\hline & \multicolumn{5}{|c|}{ Energy $(\mathrm{MeV})$} \\
\hline & 207 & 225 & 245 & 266 & 286 \\
\hline$\sigma\left(6^{+}\right)$ & \multirow{2}{*}{0.57} & \multirow{2}{*}{0.47} & \multirow{2}{*}{0.28} & \multirow{2}{*}{0.25} & \multirow{2}{*}{0.26} \\
\hline \multirow[t]{4}{*}{$\sigma\left(6^{+}\right)+\sigma\left(1^{-}\right)$} & & & & & \\
\hline & \multicolumn{5}{|c|}{${ }^{44} \mathrm{Ca}$} \\
\hline & \multicolumn{5}{|c|}{ Energy (MeV) } \\
\hline & 232 & 252 & 275 & 297 & 319 \\
\hline$\sigma\left(6^{+}\right)$ & \multirow{2}{*}{0.38} & \multirow{2}{*}{0.29} & \multirow{2}{*}{0.15} & \multirow{2}{*}{0.19} & \multirow{2}{*}{$>0.16$} \\
\hline$\sigma\left(6^{+}\right)+\sigma\left(1^{-}\right)$ & & & & & \\
\hline
\end{tabular}


Table III.16. Neutron-to-proton ratios, $N / Z$, and peaks of isotopic distributions for the interactions of $84-\mathrm{MeV}$ (1.16 X Coulomb barrier ${ }^{12} \mathrm{C}$ ions, $253-\mathrm{MeV}$ (1.08 X Coulomb barrier) ${ }^{40} \mathrm{Ca}$ ions, $207-\mathrm{MeV}$ (1.17 X Coulomb barrier) ${ }^{31} \mathrm{P}$ ions, $275-\mathrm{MeV}(1.16 \mathrm{X}$ Coulomb barrier) ${ }^{44} \mathrm{Ca}$ ions, $245-\mathrm{MeV}$ (1.16 X Coulomb barrier) ${ }^{40} \mathrm{Ar}$ ions, $113-\mathrm{MeV}$ (1.20 X Coulomb barrier) ${ }^{18} \mathrm{O}$ ions, $280-\mathrm{MeV}$ (1.19 X Coulomb barrier) ${ }^{48} \mathrm{Ca}$ ions, and $805-\mathrm{MeV}\left(1.13 \mathrm{X}\right.$ Coulomb barrier) ${ }^{132} \mathrm{Xe}$ ions with ${ }^{248} \mathrm{Crn}$ targets. Data for $40,48 \mathrm{Ca}$ taken from [HOF8'2], ${ }^{31} \mathrm{P}$ taken from [LEY90A], ${ }^{44} \mathrm{Ca}$ taken from [TUR90], ${ }^{40} \mathrm{Ar}$ taken from [LEY90], ${ }^{18} \mathrm{O}$ taken from [LEE83], and ${ }^{132} \mathrm{Xe}$ taken from [WEL87].

\begin{tabular}{|c|c|c|c|c|c|}
\hline \multirow[t]{2}{*}{ Projectile } & \multirow[t]{2}{*}{$N / Z$} & \multicolumn{4}{|c|}{ Peak of Isotopic Distributions } \\
\hline & & $\mathrm{Bk}$ & $\begin{array}{c}\text { Cf } \\
\text { (Mass }\end{array}$ & $\begin{array}{c}\text { Es } \\
\text { amber) }\end{array}$ & $\mathrm{Fm}$ \\
\hline${ }^{12} \mathrm{C}$ & 1.00 & 248 & 250 & 251 & 253 \\
\hline${ }^{40} \mathrm{Ca}$ & 1.00 & 247 & 248 & 250 & $251-252$ \\
\hline $31 \mathrm{P}$ & 1.07 & 248 & 250 & 251 & 252 \\
\hline${ }^{44} \mathrm{Ca}$ & 1.20 & 248 & 250 & 251 & $\leq 252$ \\
\hline $40 \mathrm{Ar}$ & 1.22 & 248 & 250 & 251 & $\leq 252$ \\
\hline $18 \mathrm{O}$ & 1.25 & $249-250$ & 250 & $\leq 252$ & 254 \\
\hline${ }^{48} \mathrm{Ca}$ & 1.40 & 249 & 250 & 252 & $254-255$ \\
\hline $132 \mathrm{Xe}$ & 1.44 & $249-250$ & 250 & 251 & 253 \\
\hline
\end{tabular}


Table III.17. Neutron-to-proton ratios, $N / Z$, and full-widths-at-half-maximum of isotopic distributions from the interactions of $84-\mathrm{MeV}$ (1.16 X Coulomb barrier ${ }^{12} \mathrm{C}$ ions, 253 $\mathrm{MeV}$ (1.08 X Coulomb barrier) ${ }^{40} \mathrm{Ca}$ ions, $207-\mathrm{MeV}\left(1.17 \mathrm{X}\right.$ Coulomb barrier) ${ }^{31} \mathrm{P}$ ions, 275-MeV (1.16 X Coulomb barrier) ${ }^{44} \mathrm{Ca}$ ions, $245-\mathrm{MeV}$ (1.16 X Coulomb barrier) ${ }^{40} \mathrm{Ar}$ ions, $113-\mathrm{MeV}$ (1.20 X Coulomb barrier) ${ }^{18} \mathrm{O}$ ions, $280-\mathrm{MeV}$ (1.19 X Coulomb barrier) ${ }^{48} \mathrm{Ca}$ ions, and $805-\mathrm{MeV}\left(1.13 \mathrm{X}\right.$ Coulomb barrier) ${ }^{132} \mathrm{Xe}$ ions with ${ }^{248} \mathrm{Cm}$ targets. Data for ${ }^{40,48} \mathrm{Ca}$ taken ii om [HOF82], ${ }^{31} \mathrm{P}$ taken from [LEY90A], ${ }^{44} \mathrm{Ca}$ taken from [TUR90], ${ }^{40} \mathrm{Ar}$ taken from [LEY90], ${ }^{18} \mathrm{O}$ taken from [LEE83], and ${ }^{132} \mathrm{Xe}$ taken from [WEL87].

\begin{tabular}{|c|c|c|c|c|c|}
\hline \multirow[t]{2}{*}{ Projectile } & \multirow[t]{2}{*}{$N / Z$} & \multicolumn{4}{|c|}{ Full-Width-At-Half-Maximum } \\
\hline & & \multicolumn{4}{|c|}{ (Mass Number) } \\
\hline${ }^{12} \mathrm{C}$ & 1.00 & 2.1 & 1.8 & $<1.5$ & 1.8 \\
\hline${ }^{40} \mathrm{Ca}$ & 1.00 & 2.3 & 2.5 & 2.3 & 2.6 \\
\hline $31 p$ & 1.07 & 2.5 & 2.5 & $<1.5$ & 2.3 \\
\hline${ }^{44} \mathrm{Ca}$ & 1.20 & 3.0 & 3.5 & 2.0 & $-\cdots$ \\
\hline${ }^{40} \mathrm{Ar}$ & 1.22 & 3.3 & 2.8 & 1.5 & $\cdots$ \\
\hline $18 \mathrm{O}$ & 1.25 & 2.2 & 2.8 & --- & 3.0 \\
\hline${ }^{48} \mathrm{Ca}$ & 1.40 & $\cong 3.8$ & 2.0 & ... & 2.0 \\
\hline $132 \mathrm{Xe}$ & 1.44 & $\cong 3.8$ & 2.0 & 2.2 & $\cong 2.7$ \\
\hline
\end{tabular}




\section{Figure Captions}

Figure III.1. Isotopic distributions for $\mathrm{Bk}, \mathrm{Cf}, \mathrm{Es}$, and $\mathrm{Fm}$ from the reactions of 84$\mathrm{MeV}\left(1.16 \mathrm{X}\right.$ Coulomb barrier) ${ }^{12} \mathrm{C}$ ions with ${ }^{248} \mathrm{Cm}$.

Figure III.2. Isotopic distributions for $\mathrm{Bk}, \mathrm{Cf}, \mathrm{Es}$, and $\mathrm{Fm}$ from the reactions of 71$\mathrm{MeV}(0.98 \times$ Coulomb barrier $){ }^{12} \mathrm{C}$ ions with $248 \mathrm{Cm}$.

Figure III.3. Excitation functions for isotopes of $\mathrm{Bk}$ from the interactions of ${ }^{12} \mathrm{C}$ with $248 \mathrm{Cm}$.

Figure III.4. Excitation functions for isotopes of $\mathrm{Cf}$ from the interactions of ${ }^{12} \mathrm{C}$ with ${ }^{248} \mathrm{Cm}$.

Figure III.5. Excitation functions for isotopes of Es from the interactions of $12 \mathrm{C}$ with ${ }^{248} \mathrm{Cm}$.

Figure III.6. Excitation functions for isotopes of $\mathrm{Fm}$ from the interactions of ${ }^{12} \mathrm{C}$ with ${ }^{248} \mathrm{Cm}$.

Figure III.7. Isotopic distributions for $\mathrm{Bk}, \mathrm{CF}$, Es, and $\mathrm{Fm}$ from the reactions of 207$\mathrm{MeV}$ (1.17 X Coulomb barrier) ${ }^{31} \mathrm{P}$ ions with ${ }^{248} \mathrm{Cm}$. Taken from [LEY90A].

Figure III.8. Excitation functions for isotopes of $\mathrm{Bk}$ from the interactions of ${ }^{31} \mathrm{P}$ with ${ }^{248} \mathrm{Cm}$. Taken from [LEY90A].

Figure III.9. Excitation functions for isotopes of $\mathrm{Cf}$ from the interactions of $31 \mathrm{P}$ with ${ }^{248} \mathrm{Cm}$. Taken from [LEY90A].

Figure III.10. Excitation functions for isotopes of Es from the interactions of $31 \mathrm{P}$ with ${ }^{248} \mathrm{Cm}$. Taken from [LEY90A].

Figure III.11. Excitation functions for isotopes of Fm from the interactions of $31 \mathrm{P}$ with ${ }^{248} \mathrm{Cm}$. Taken from [LEY90A].

Figure III.12. Isotopic distributions for $\mathrm{Bk}, \mathrm{Cf}$, Es, and $\mathrm{Fm}$ from the interactions of 245-MeV (1.16 X Coulomb barrier) ${ }^{40} \mathrm{Ar}$ ions with ${ }^{248} \mathrm{Cm}$. Taken from [LEY90].

Figure III.13. Excitation functions for isotopes of $\mathrm{Bk}$ from the interactions of $40 \mathrm{Ar}$ with ${ }^{248} \mathrm{Cm}$. Taken from [LEY90]. 
Figure III.14. Excitation functions for isotopes of $\mathrm{Cf}$ from the interactions of $40 \mathrm{Ar}$ with ${ }^{248} \mathrm{Cm}$. Taken from [LEY90].

Figure III.15. Excitation functions for isotopes of Es from the interactions of $40 \mathrm{Ar}$ with ${ }^{248} \mathrm{Cm}$. Taken from [LEY90].

Figure III.16. Fraction of yield going to $250 \mathrm{Es} g\left(\sigma\left(6^{+}\right) /\left[\sigma\left(6^{+}\right)+\sigma\left(1^{-}\right)\right]\right)$as a function of energy for the ${ }^{40} \mathrm{Ar}-{ }^{248} \mathrm{Cm}$ sysisem.

Figure III.17. Excitation functions for isotopes of Fm from the interactions of $40 \mathrm{Ar}$ with ${ }^{248} \mathrm{Cm}$. Taken from [LEY90].

Figure III.18. Isotopic distributions for $\mathrm{Bk}, \mathrm{Cf}, \mathrm{Es}$, and $\mathrm{Fm}$ from the interactions of 275-MeV (1.16 X Coulomb barrier) ${ }^{44} \mathrm{Ca}$ ions with ${ }^{248} \mathrm{Cm}$. Taken from [LEY90].

Figure III.19. Excitation functions for isotopes of $\mathrm{Bk}$ from the interastions of ${ }^{44} \mathrm{Ca}$ with ${ }^{248} \mathrm{Cm}$. Taken from [LEY90].

Figure III.20. Excitation functions for isotopes of Cf from the interactions of ${ }^{44} \mathrm{Ca}$ with ${ }^{248} \mathrm{Cm}$. Taken from [LEY90].

Figure III.21. Excitation functions for isotopes of Es from the interactions of ${ }^{44} \mathrm{Ca}$ with ${ }^{248} \mathrm{Cm}$. Taken from [LEY90].

Figure III.22. Fraction of yield going to $250 \mathrm{Es} g\left(\sigma\left(6^{+}\right) /\left[\sigma\left(6^{+}\right)+\sigma\left(1^{-}\right)\right]\right)$as a function of energy for the ${ }^{44} \mathrm{Ca}-{ }^{248} \mathrm{Cm}$ system.

Figure III.23. Excitation functions for isotopes of $\mathrm{Fm}$ from the interactions of ${ }^{44} \mathrm{Ca}$ with ${ }^{248} \mathrm{Cm}$. Taken from [LEY90].

Figure III.24. Isotopic distributions for Bk produced in the interactions of $84-\mathrm{MeV}$ (1.16 X Coulomb barrier) ${ }^{12} \mathrm{C}$ ions, $207-\mathrm{MeV}\left(1.17 \mathrm{X}\right.$ Coulomb barrier) ${ }^{31} \mathrm{P}$ ions, $245-$ $\mathrm{MeV}\left(1.16 \mathrm{X}\right.$ Coulomb barrier) ${ }^{40} \mathrm{Ar}$ ions, $253-\mathrm{MeV}\left(1.08 \mathrm{X}\right.$ Coulomb barrier) ${ }^{40} \mathrm{Ca}$ ions, $275-\mathrm{MeV}$ (1.16 X Coulomb barrier) ${ }^{44} \mathrm{Ca}$ ions, and $280-\mathrm{MeV}$ (1.19 X Coulomb barrier) ${ }^{48} \mathrm{Ca}$ ions with ${ }^{248} \mathrm{Cm} . \Delta \mathrm{N}$ is the total number of neutrons transferred. $\mathrm{A}(+)$ sign indicates that neutrons are transferred from the projectile to the target, while a (-) sign indicates neutron transfer in the opposite direction. 
Figure III.25. Isotopic distributions for Cf produced in the interactions of $84-\mathrm{MeV}$ (1.16 X Coulomb barrier) ${ }^{12} \mathrm{C}$ ions, $207-\mathrm{MeV}\left(1.17 \mathrm{X}\right.$ Coulomb barrier) ${ }^{31} \mathrm{P}$ ions, 245 $\mathrm{MeV}\left(1.16 \mathrm{X}\right.$ Coulomb barrier) ${ }^{40} \mathrm{Ar}$ ions, $253 \mathrm{-MeV}(1.08 \times$ Coulomb barrier $){ }^{40} \mathrm{Ca}$ ions, $275-\mathrm{MeV}$ (1.16 X Coulomb barrier) ${ }^{44} \mathrm{Ca}$ ions, and $280-\mathrm{MeV}(1.19 \mathrm{X}$ Coulomb barrier) ${ }^{48} \mathrm{Ca}$ ions with ${ }^{248} \mathrm{Cm} . \Delta N$ is the total number of neutrons transferred. A ( + ) sign indicates that neutrons are transferred from the projectile to the target, while a (-) sign indicates neutron transfer in the opposite direction.

Figure III.26. Isotopic distributions for Es produced in the interactions of $84-\mathrm{MeV}$ (1.16 X Coulomb barrier) ${ }^{12} \mathrm{C}$ ions, $207-\mathrm{MeV}\left(1.17 \mathrm{X}\right.$ Coulomb barrier) ${ }^{31} \mathrm{P}$ ions, $245-$ $\mathrm{MeV}\left(1.16 \mathrm{X}\right.$ Coulumb barrier) ${ }^{40} \mathrm{Ar}$ ions, $253 \mathrm{-MeV}(1.08 \times$ Coulomb barrier $){ }^{40} \mathrm{Ca}$ ions, $275-\mathrm{MeV}\left(1.16 \mathrm{X}\right.$ Coulomb barrier) ${ }^{44} \mathrm{Ca}$ ions, and $280-\mathrm{MeV}(1.19 \mathrm{X}$ Coulomb barrier) ${ }^{48} \mathrm{Ca}$ ions with ${ }^{248} \mathrm{Cm} . \Delta N$ is the total number of neutrons transferred. A (+) sign indicates that neutrons are transferred from the projectile to the target, while a (-) sign indicates neutron transfer in the opposite direction.

Figure III.27. Isotopic distributions for Fm produced in the interactions of $84-\mathrm{MeV}$ (1.16 X Coulomb barrier) ${ }^{12} \mathrm{C}$ ions, $207-\mathrm{MeV}\left(1.17 \mathrm{X}\right.$ Coulomb barrier) ${ }^{31} \mathrm{P}$ ions, 245 $\mathrm{MeV}\left(1.16 \mathrm{X}\right.$ Coulomb barrier) ${ }^{40} \mathrm{Ar}$ ions, $253-\mathrm{MeV}\left(1.08 \times\right.$ Coulomb barrier) ${ }^{40} \mathrm{Ca}$ ions, 275-MeV (1.16 X Coulomb barrier) ${ }^{44} \mathrm{Ca}$ ions, and $280-\mathrm{MeV}$ (1.19 X Coulomb barrier) ${ }^{48} \mathrm{Ca}$ ions with ${ }^{248} \mathrm{Cm} . \Delta N$ is the total number of neutrons transferred. A (+) sign indicates that neutrons are transferred from the projectile to the target, while a (-) sign indicates neutron transfer in the opposite direction. 


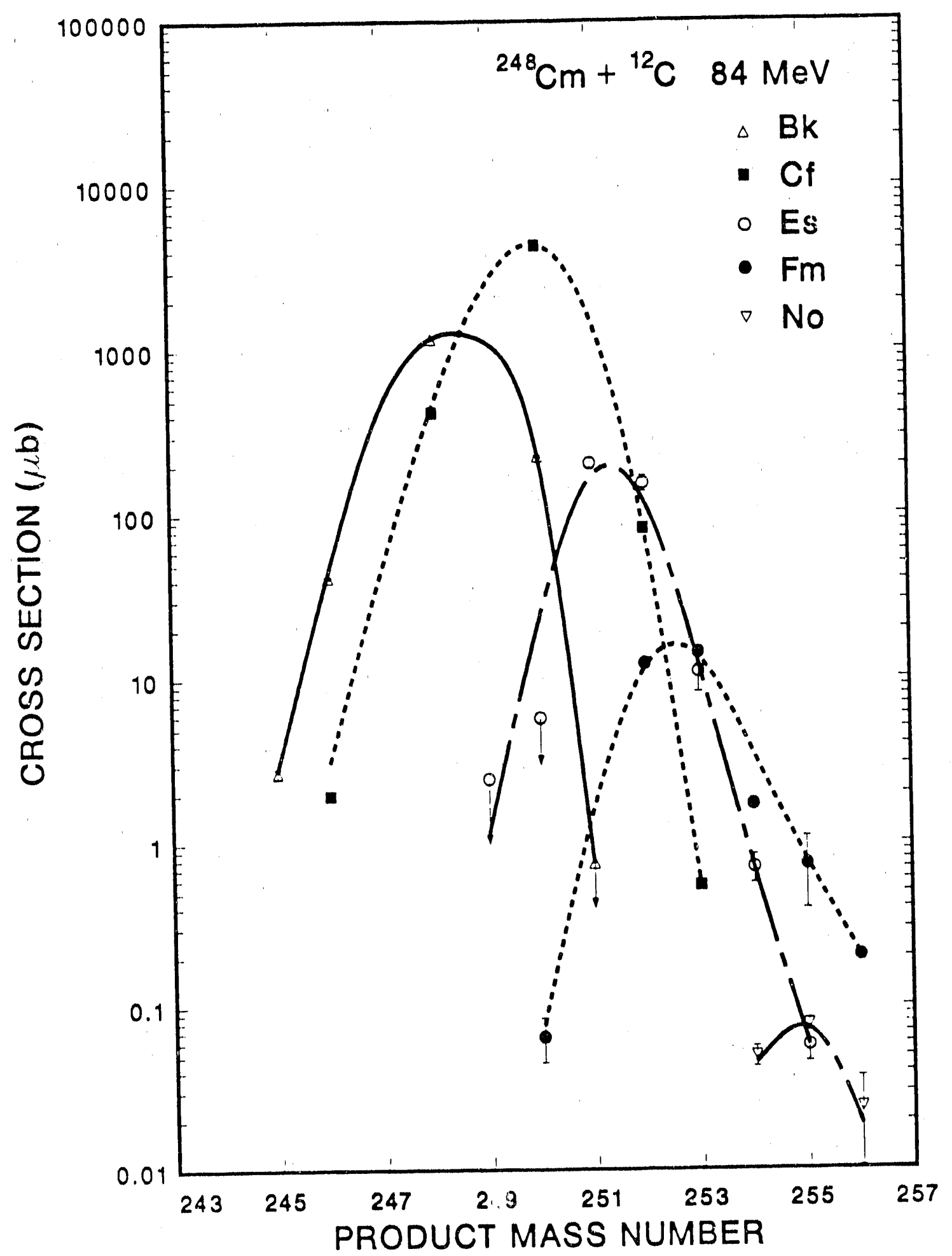

Fig. III.1 


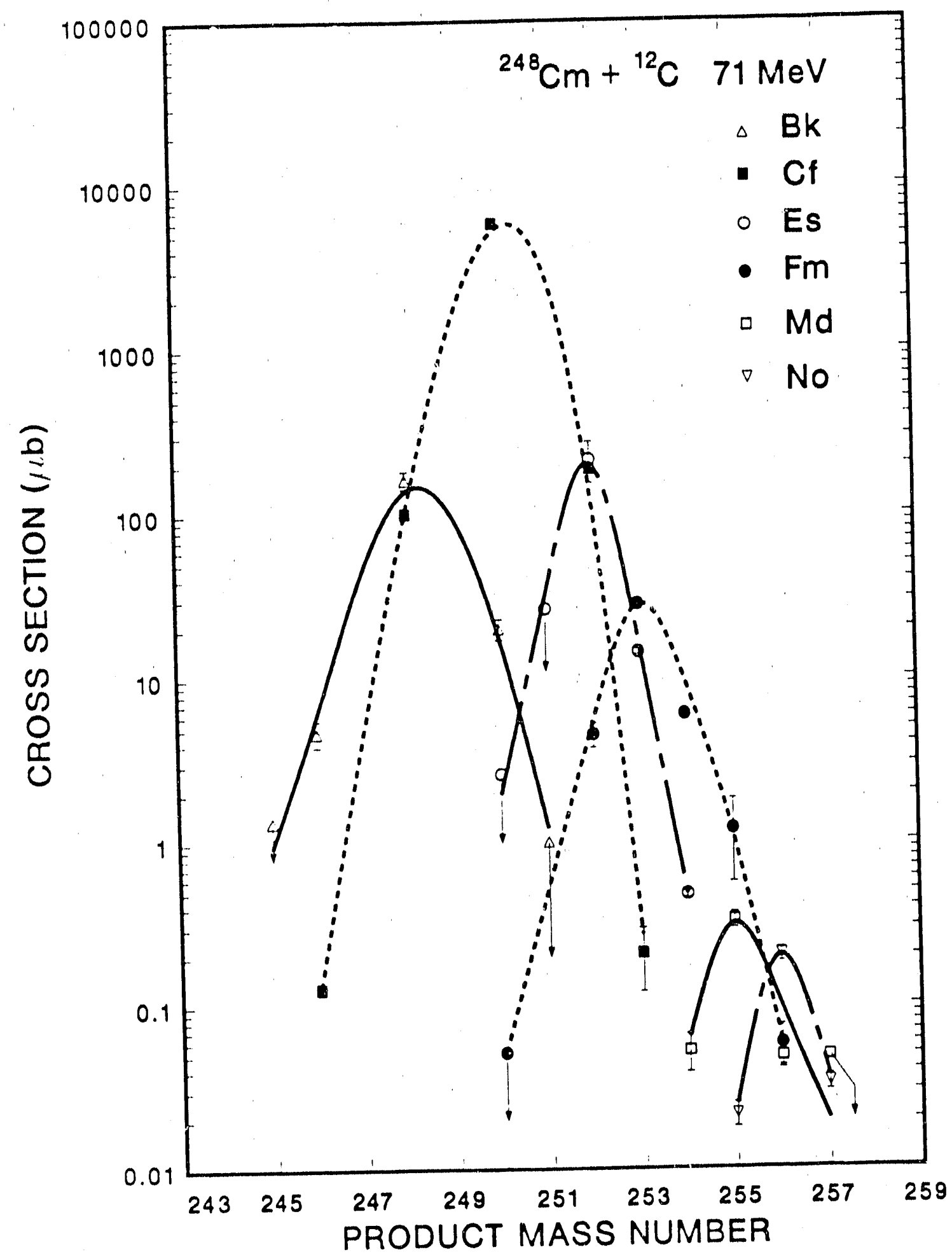

Fig. III.2 


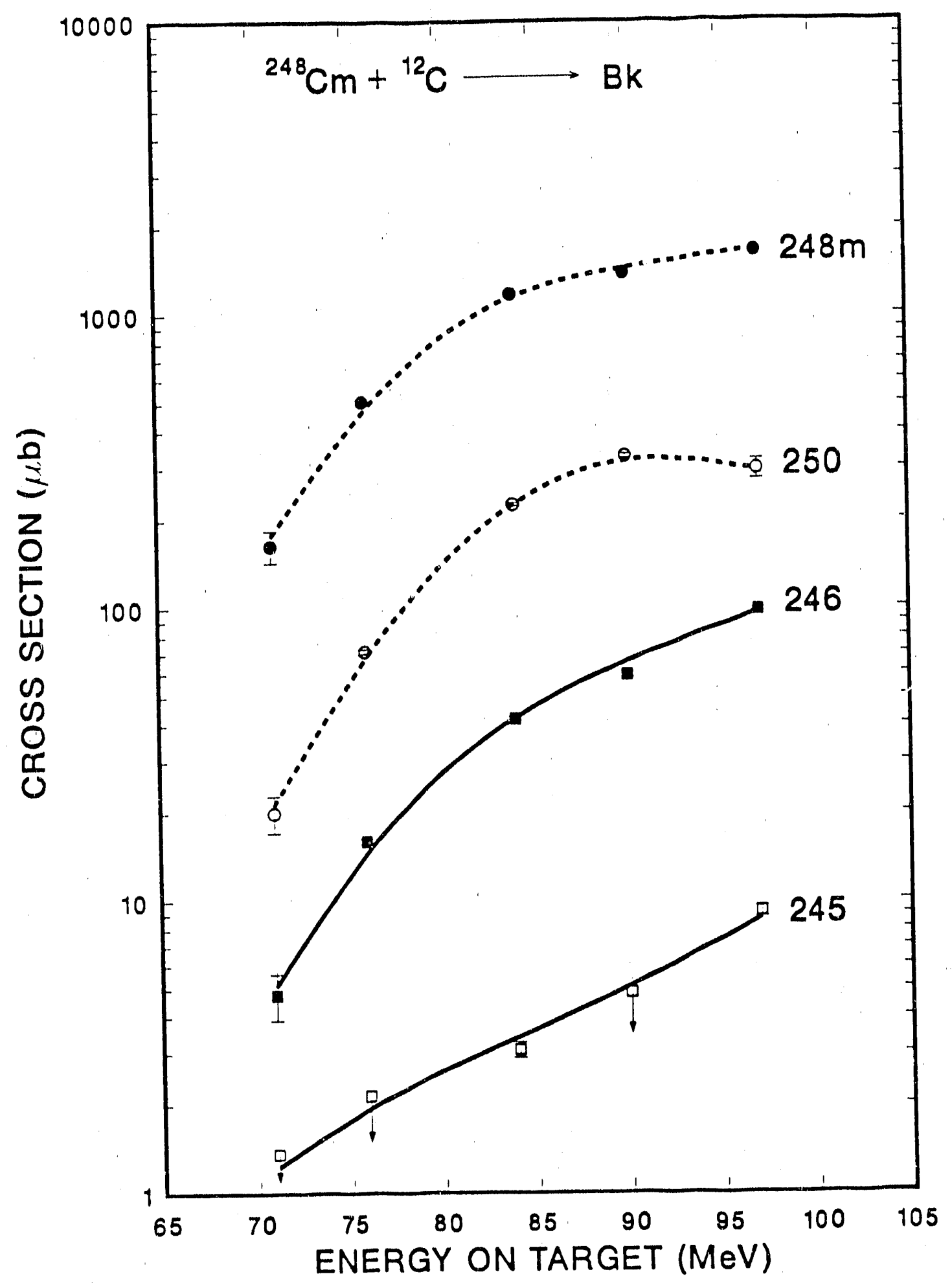

Fig. III.3 


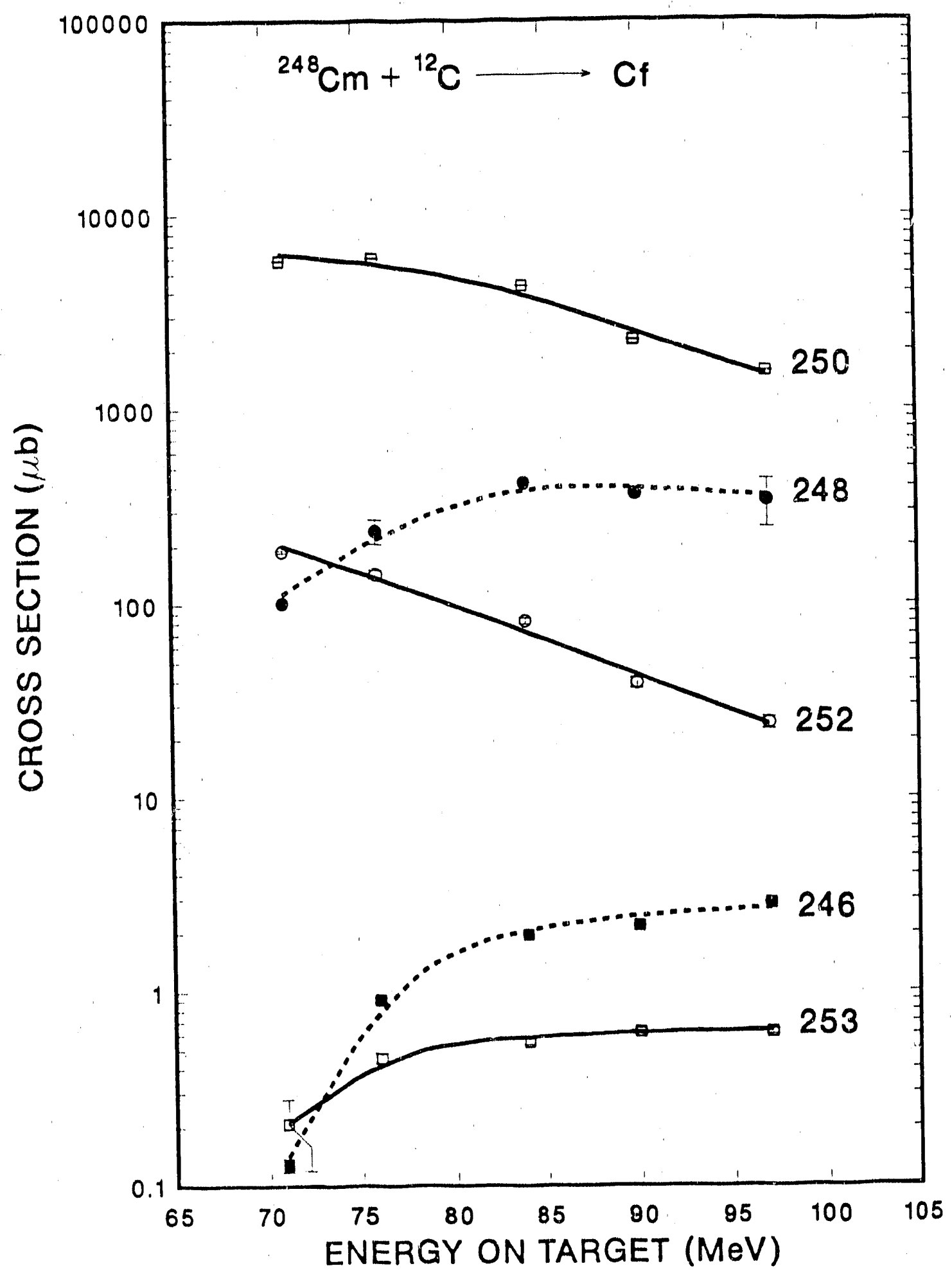

Fig. III.4 


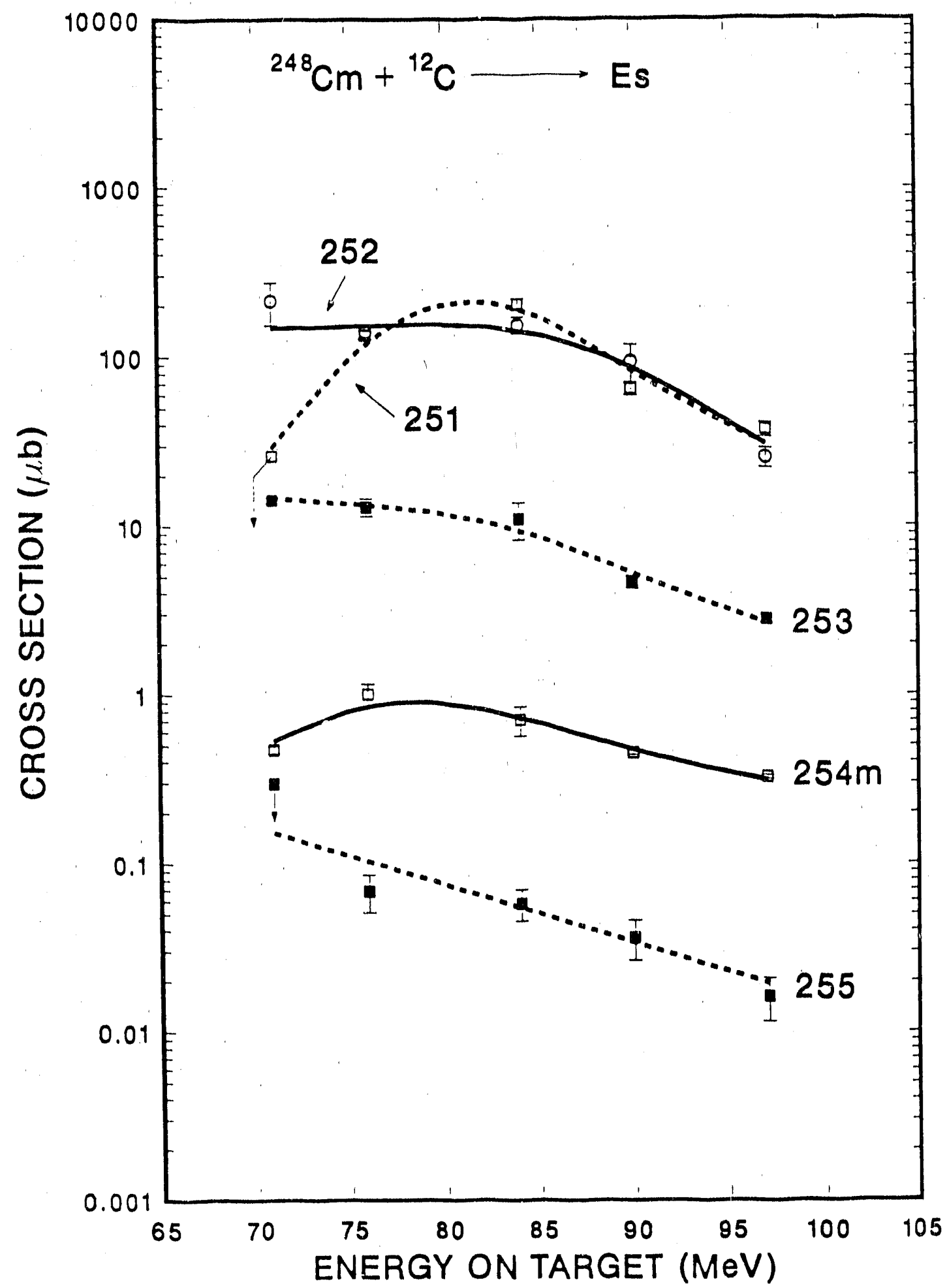

Fig. III.5 


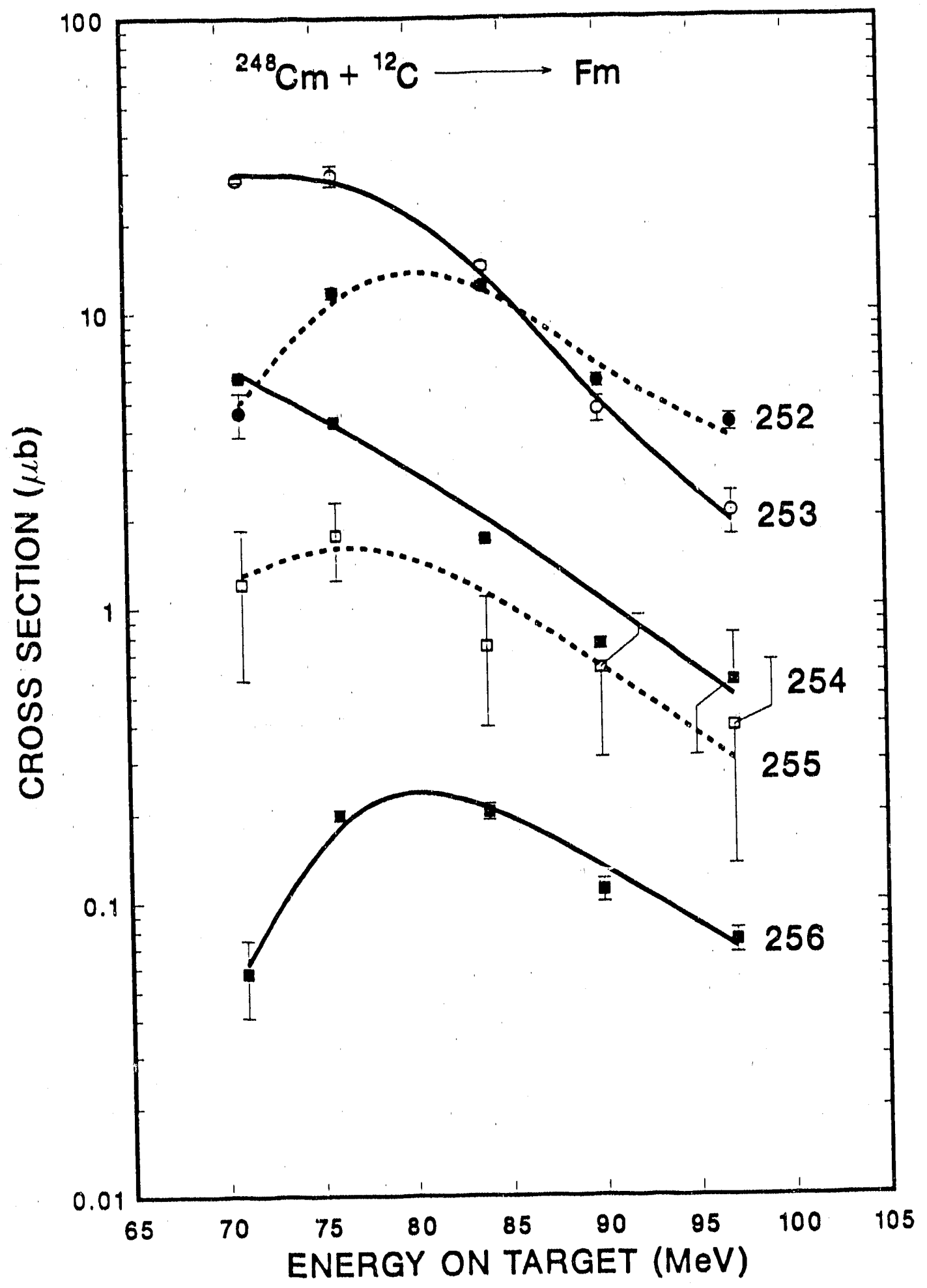

Fig. III.6 


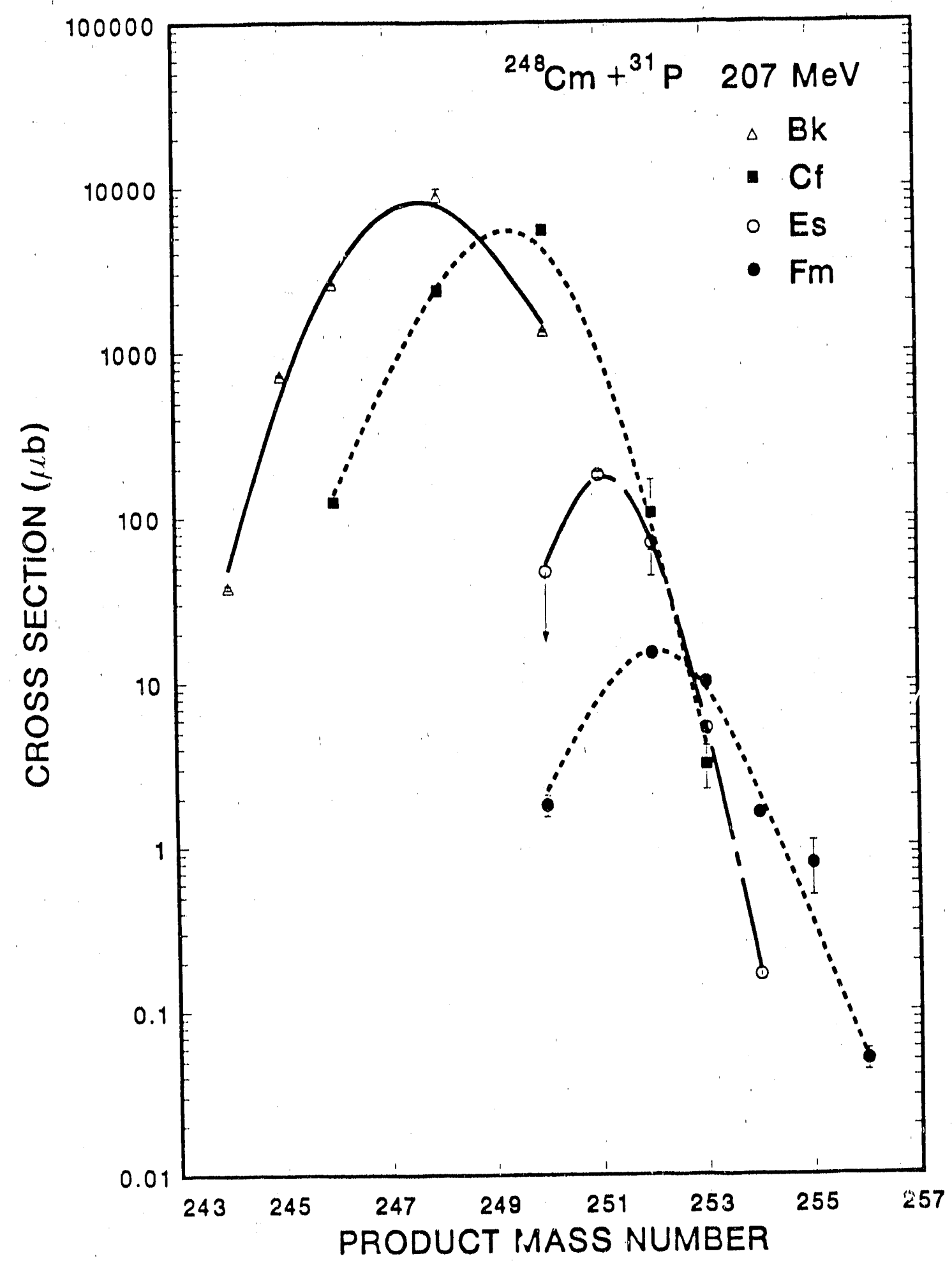

Fig. III.7 


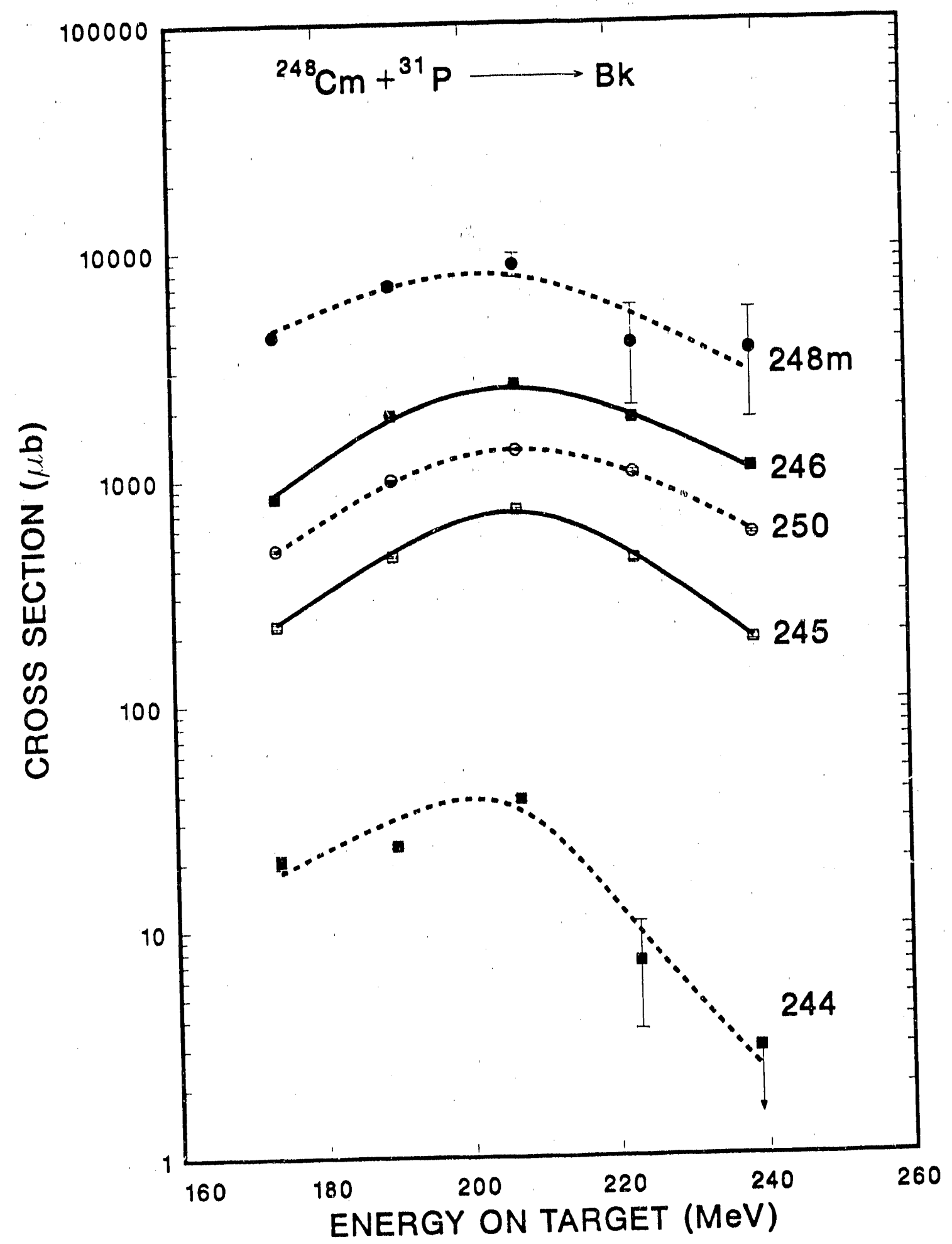

Fig. III.8 


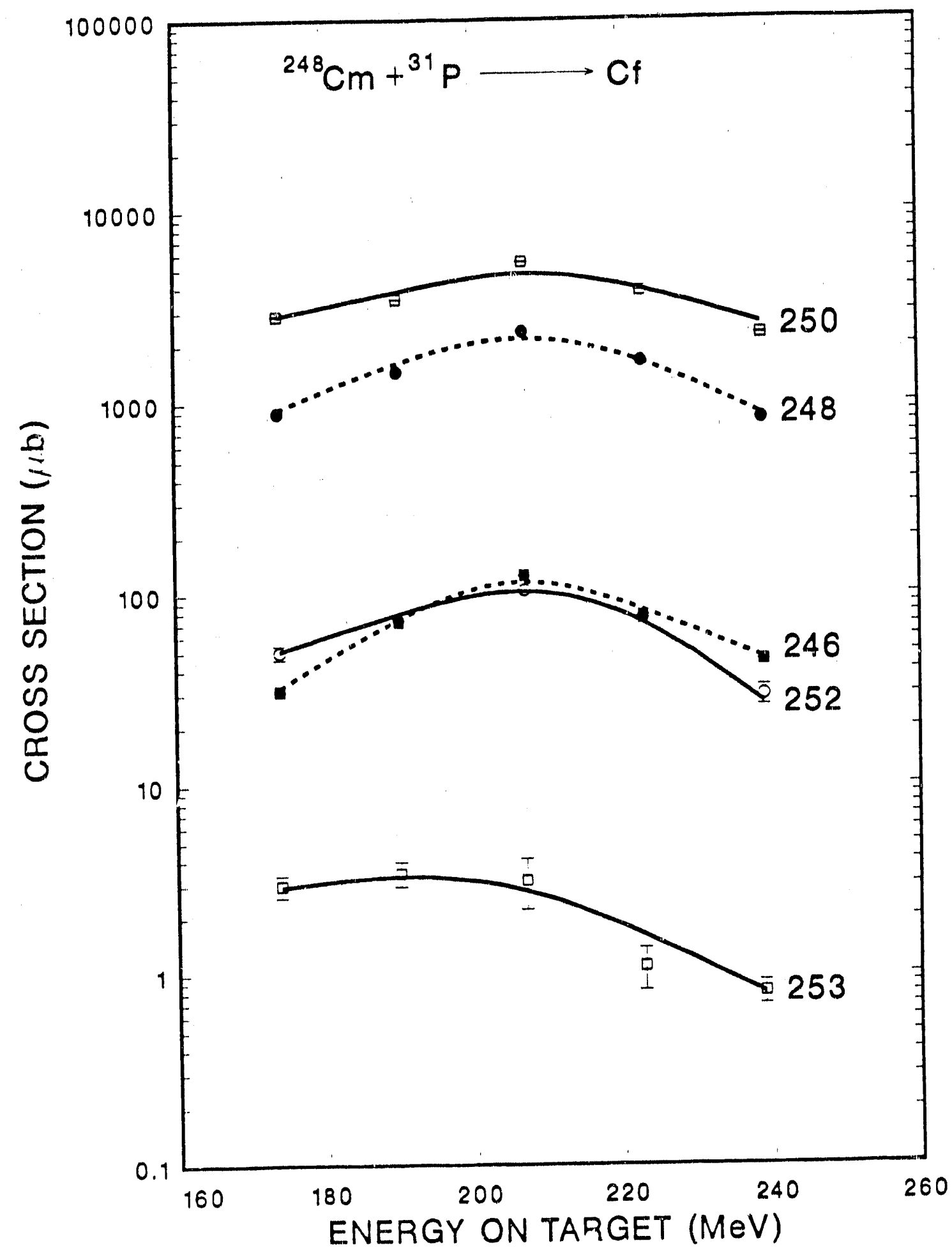

Fig. III.9 


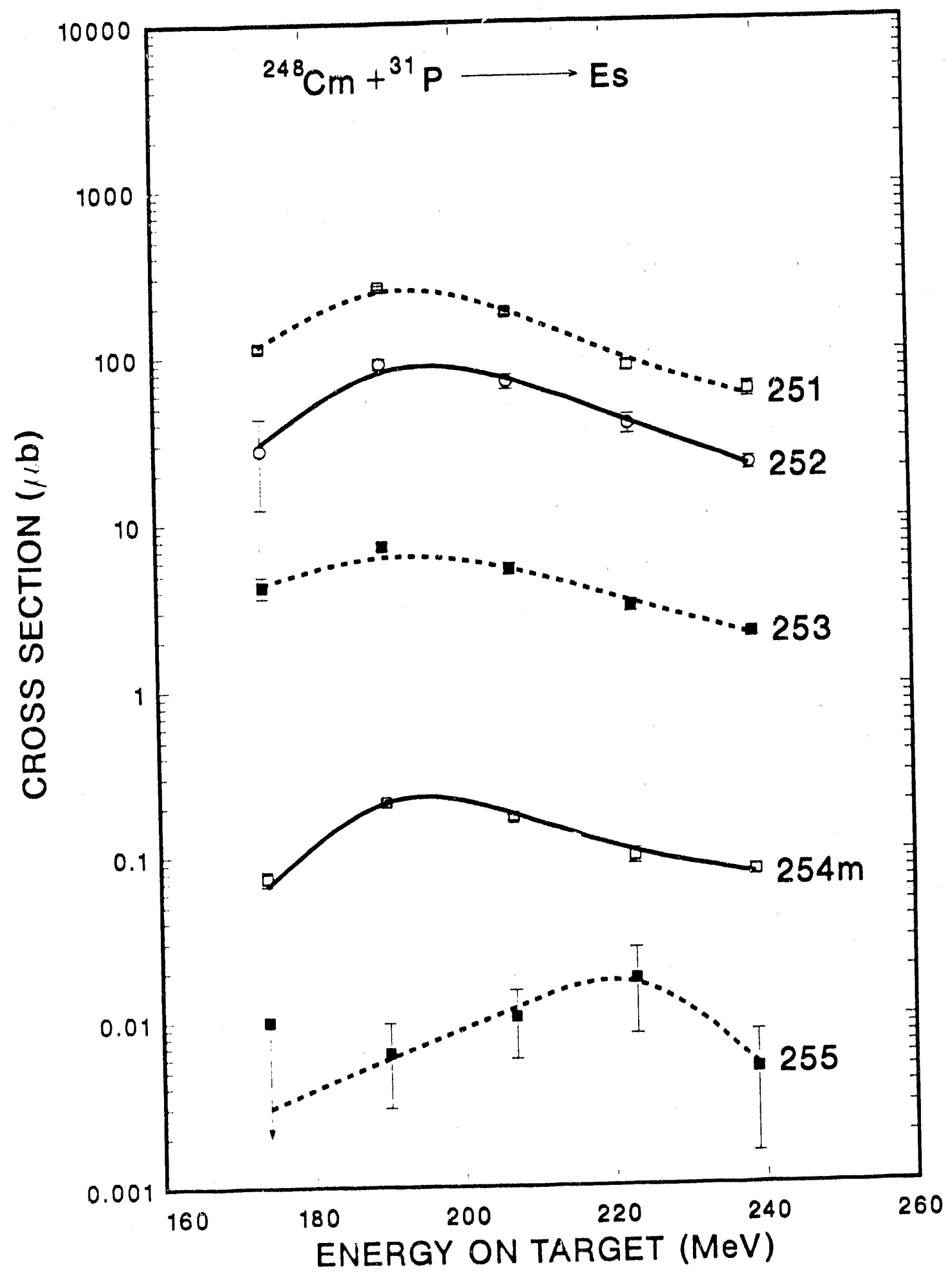

Fig. III.10 


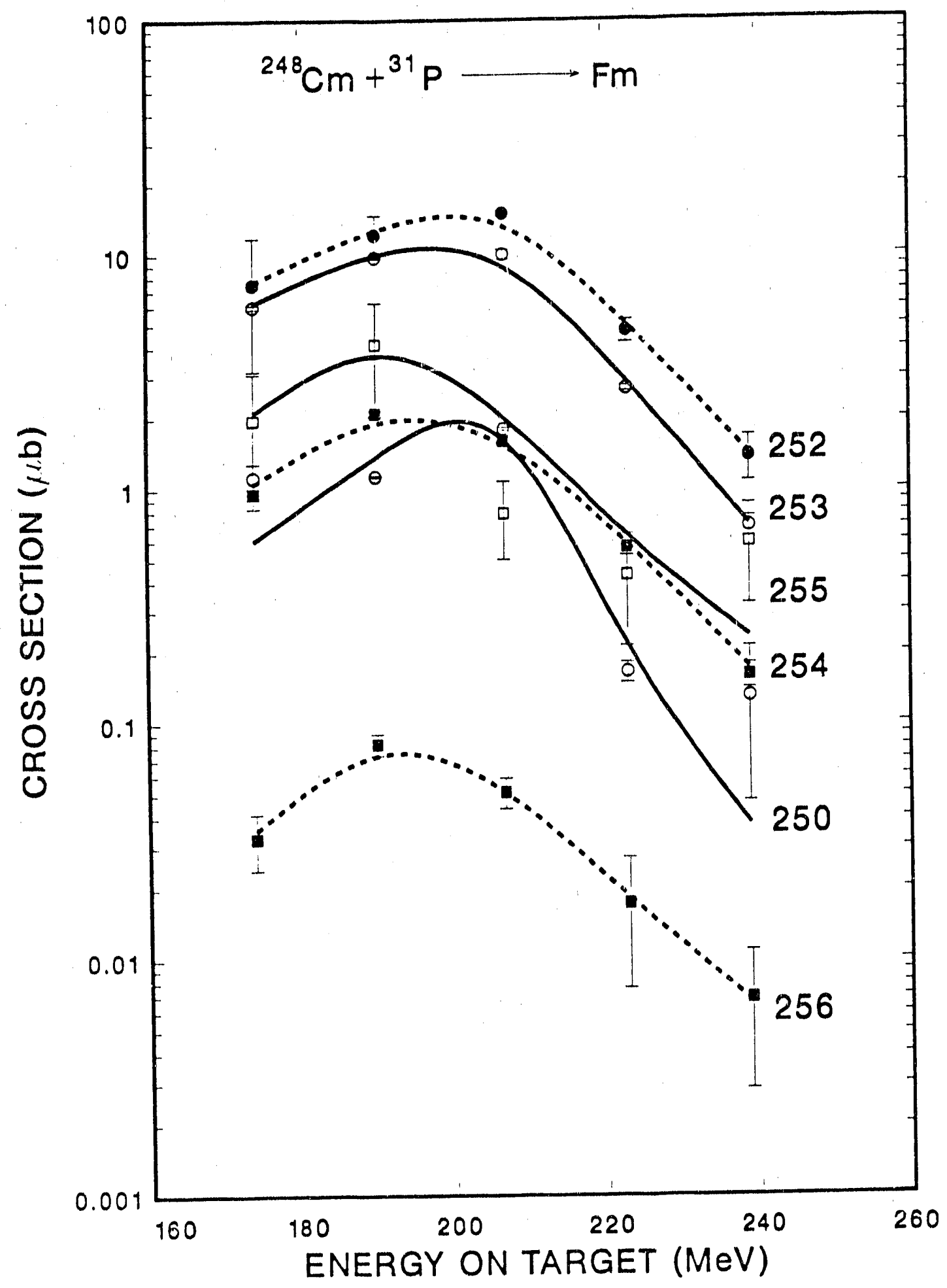

Fig. III.11 


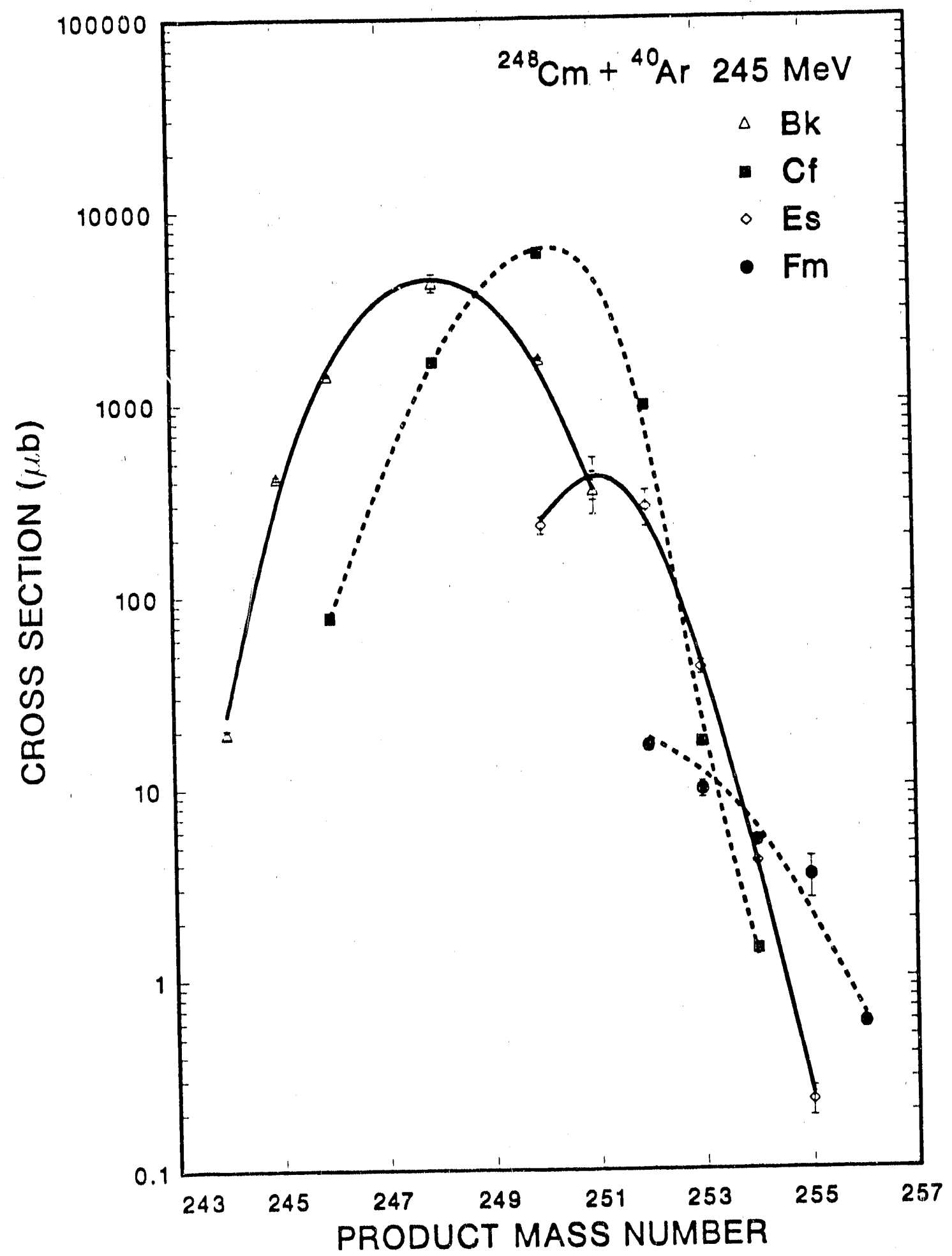

Fig. III.12 


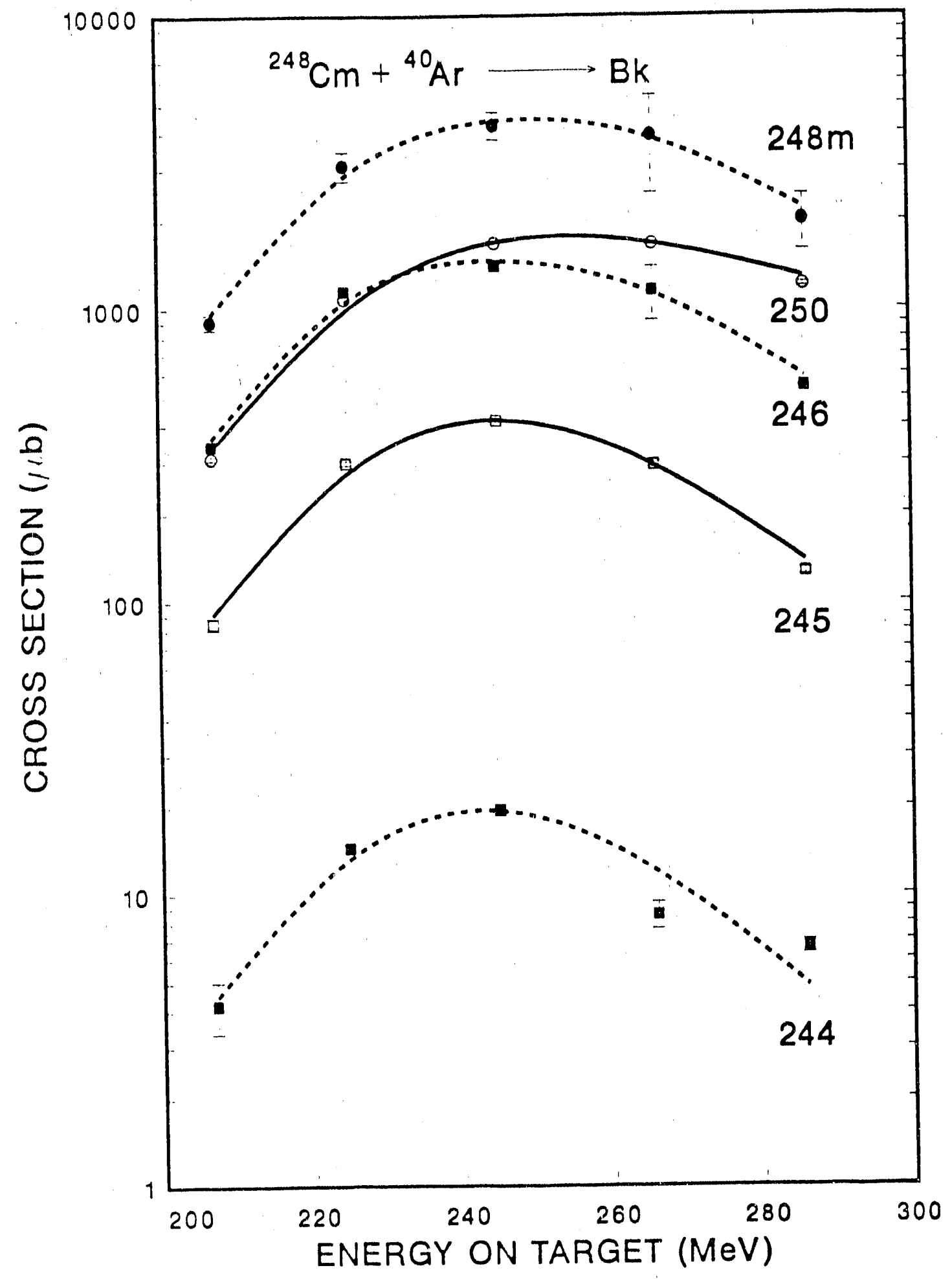

Fig. III.13 


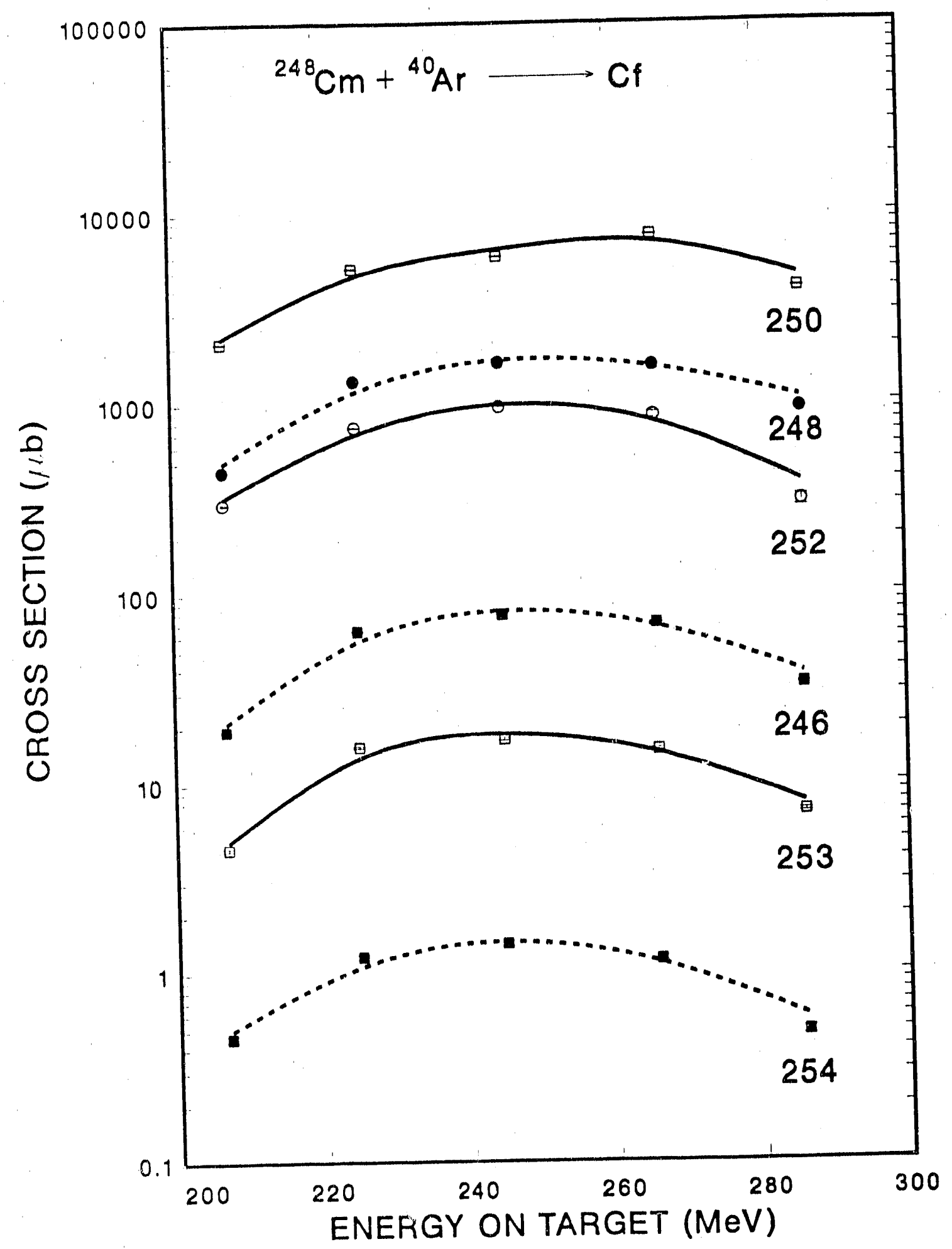

Fig. III.14 


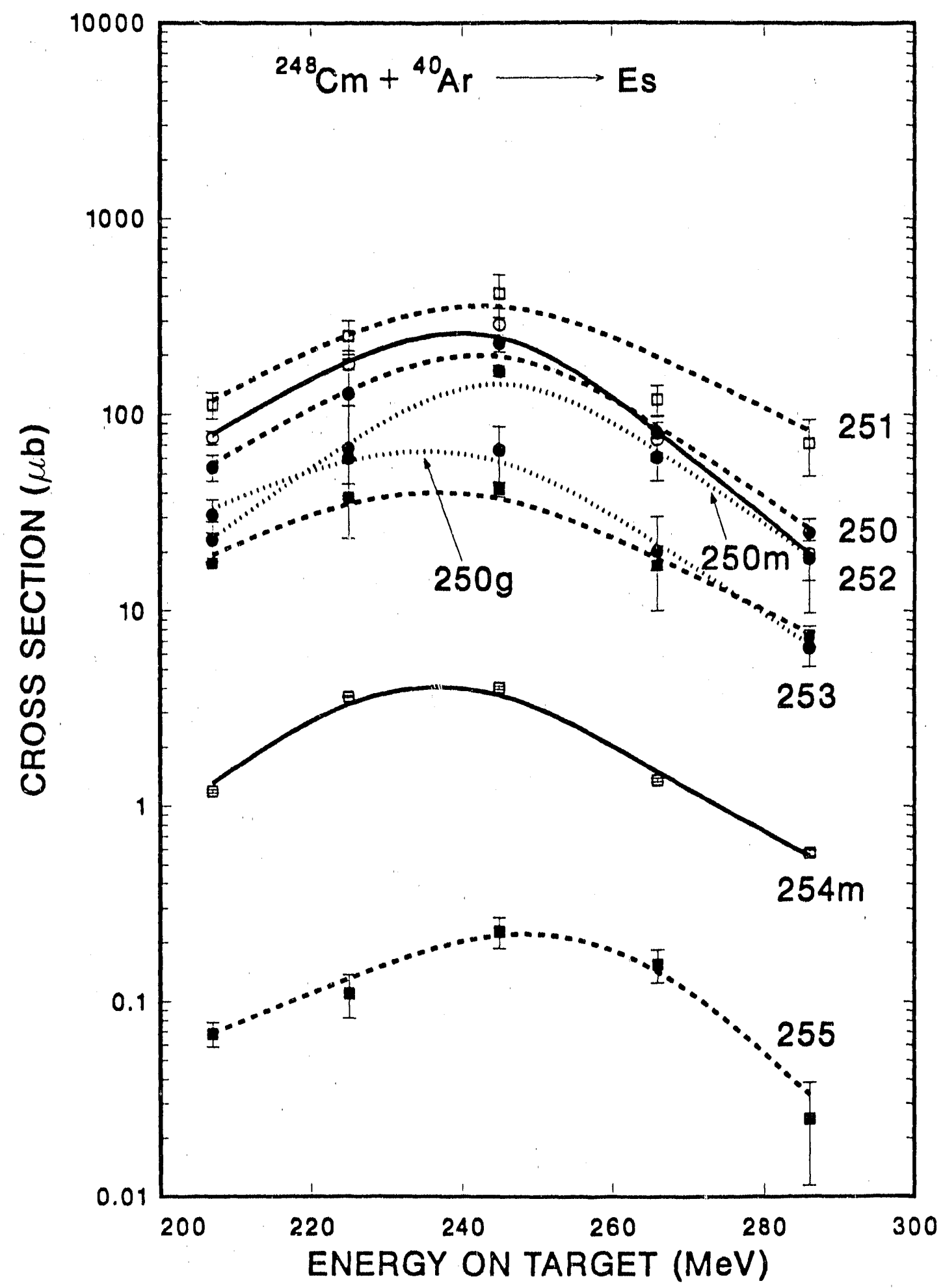

Fig. III.15 


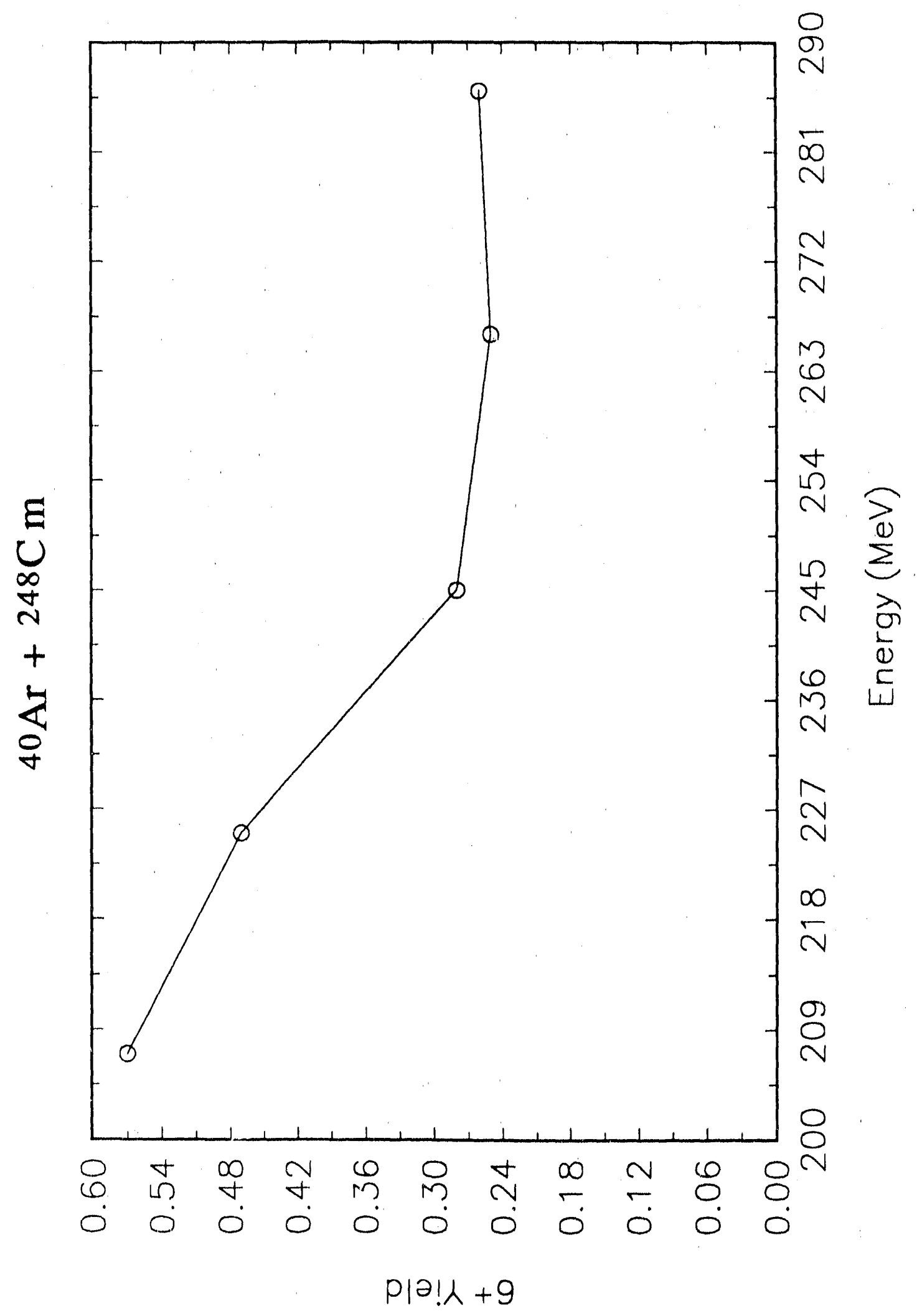

Fig. III.16 


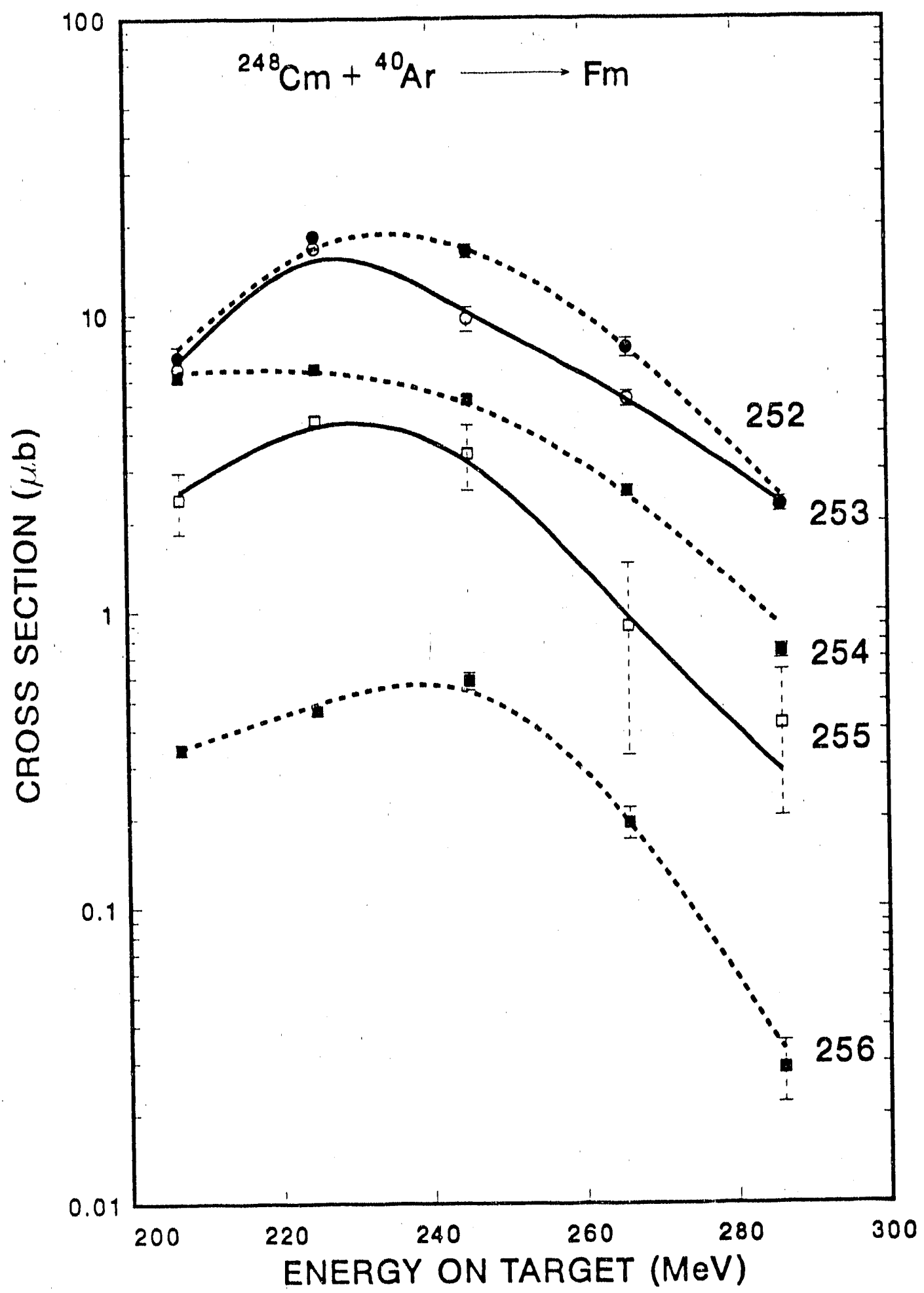

Fig. III.17 


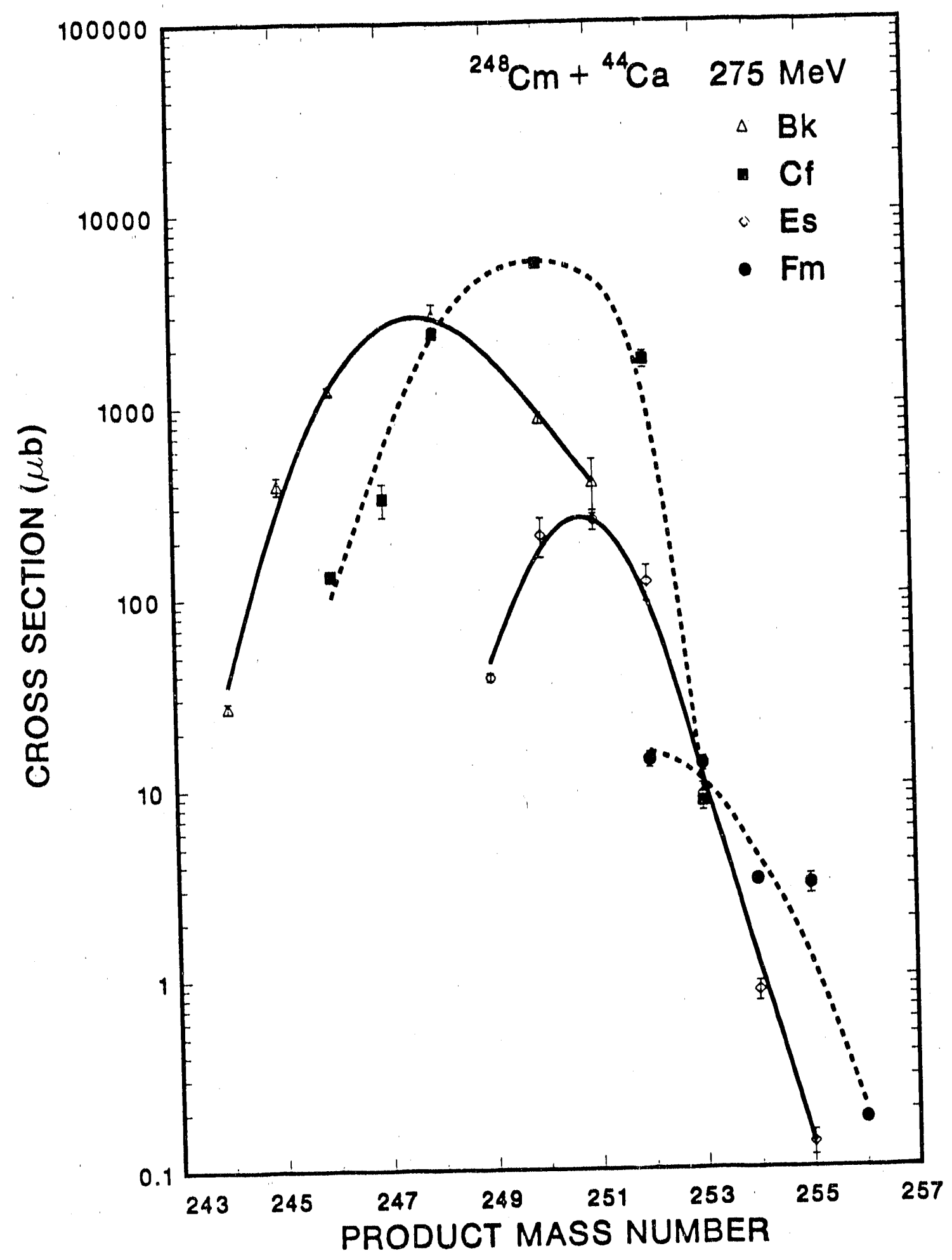

Fig. III.18 


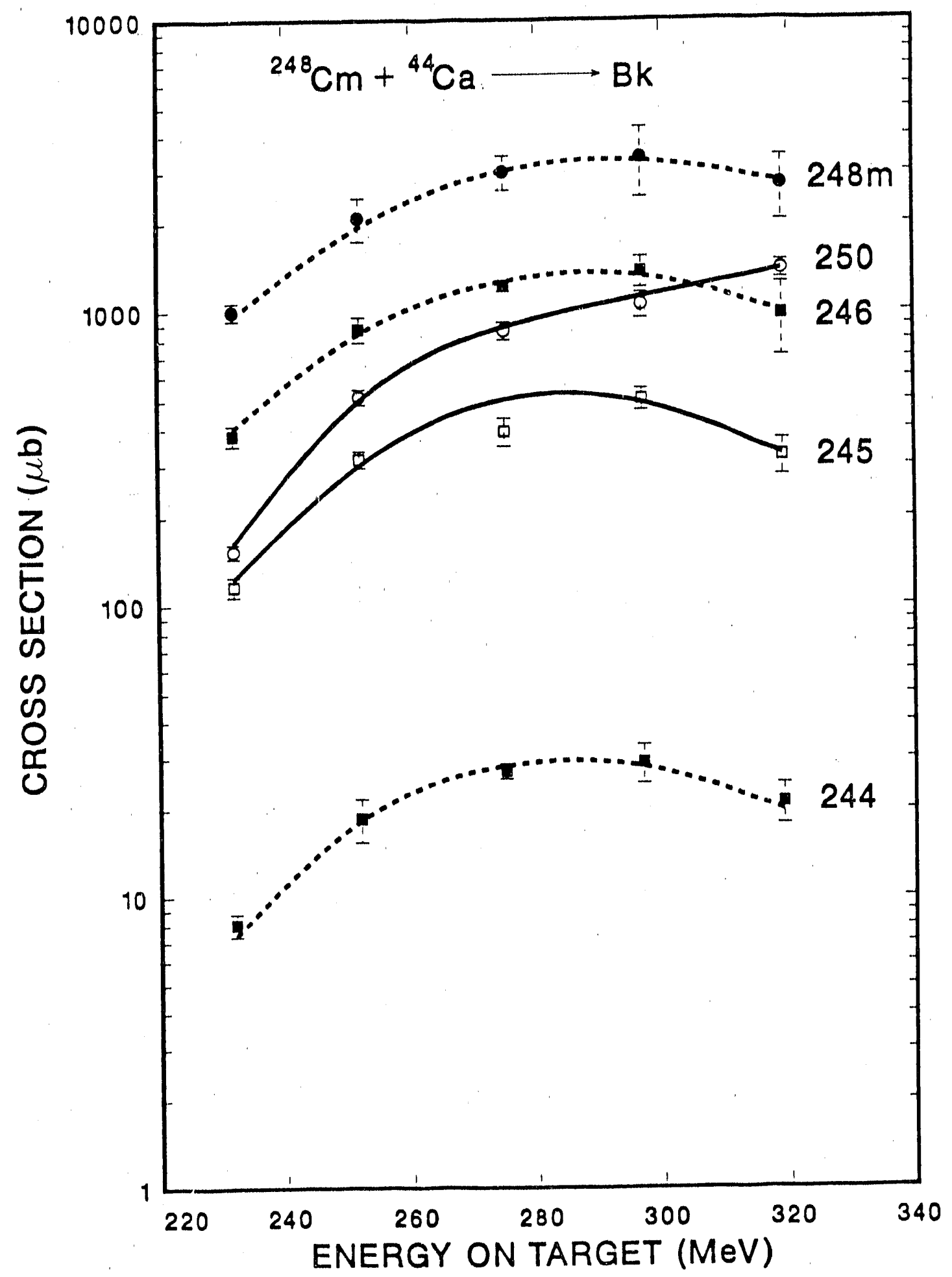

Fig. III.19 


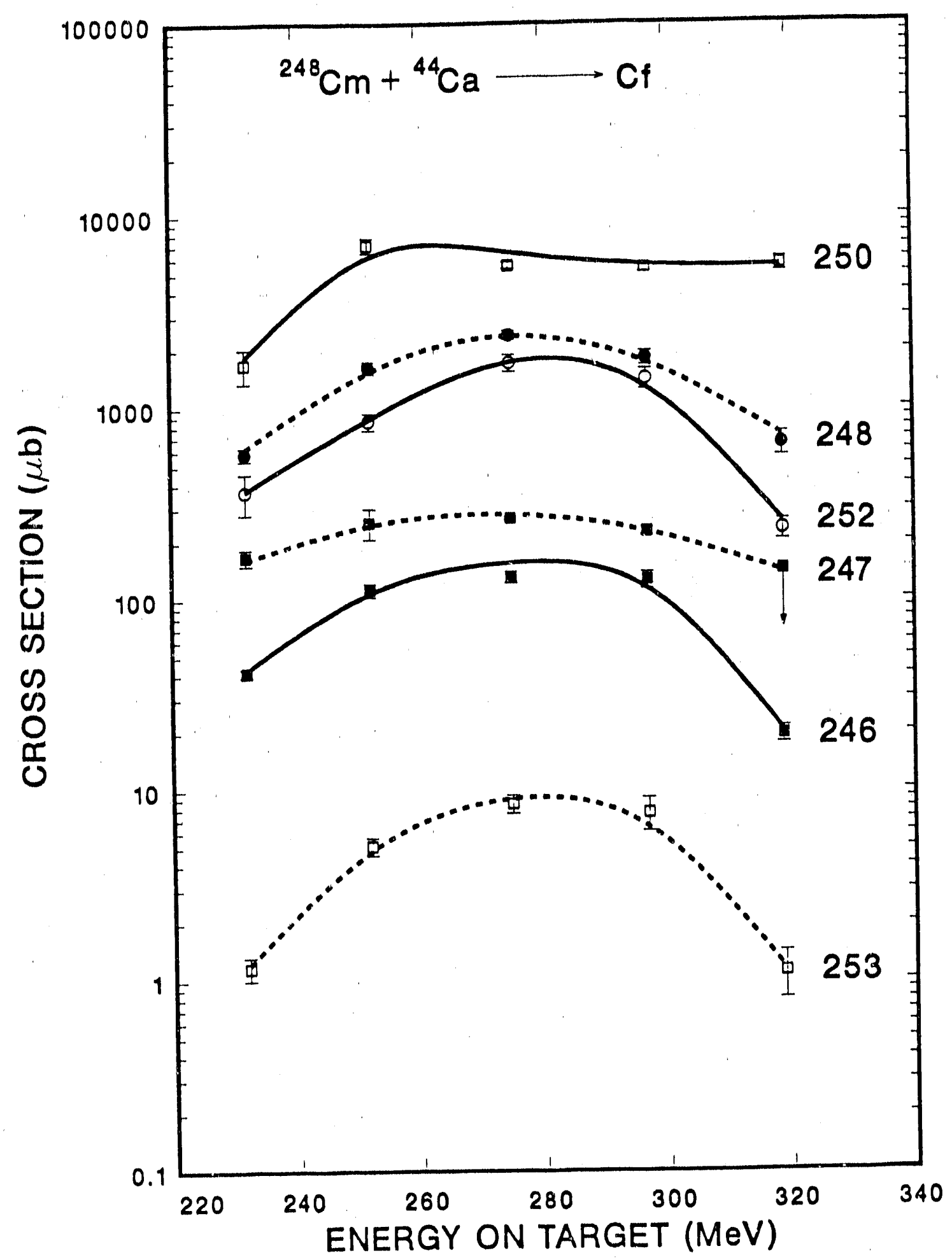

Fig. III.20 


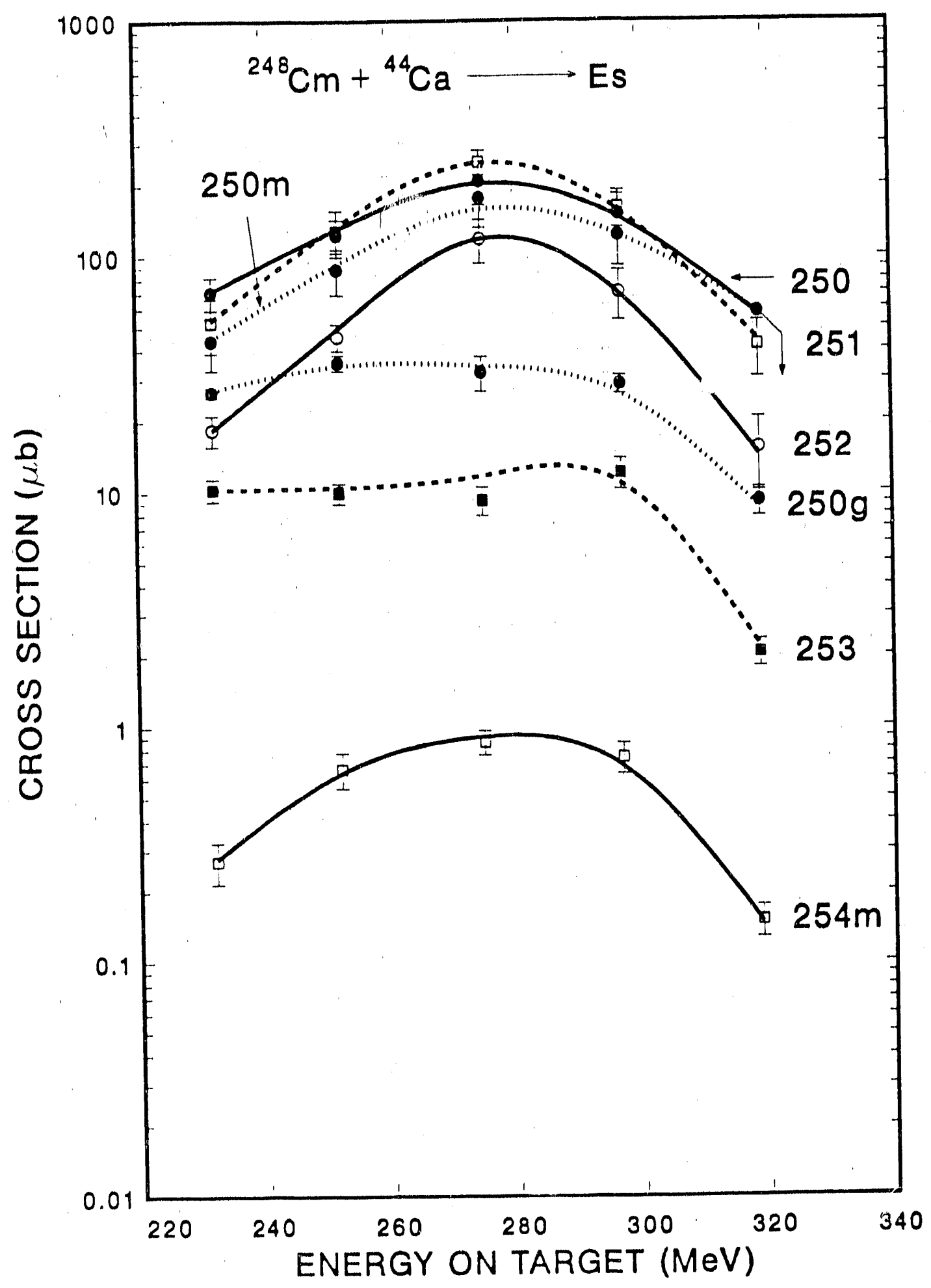

Fig. III.21 


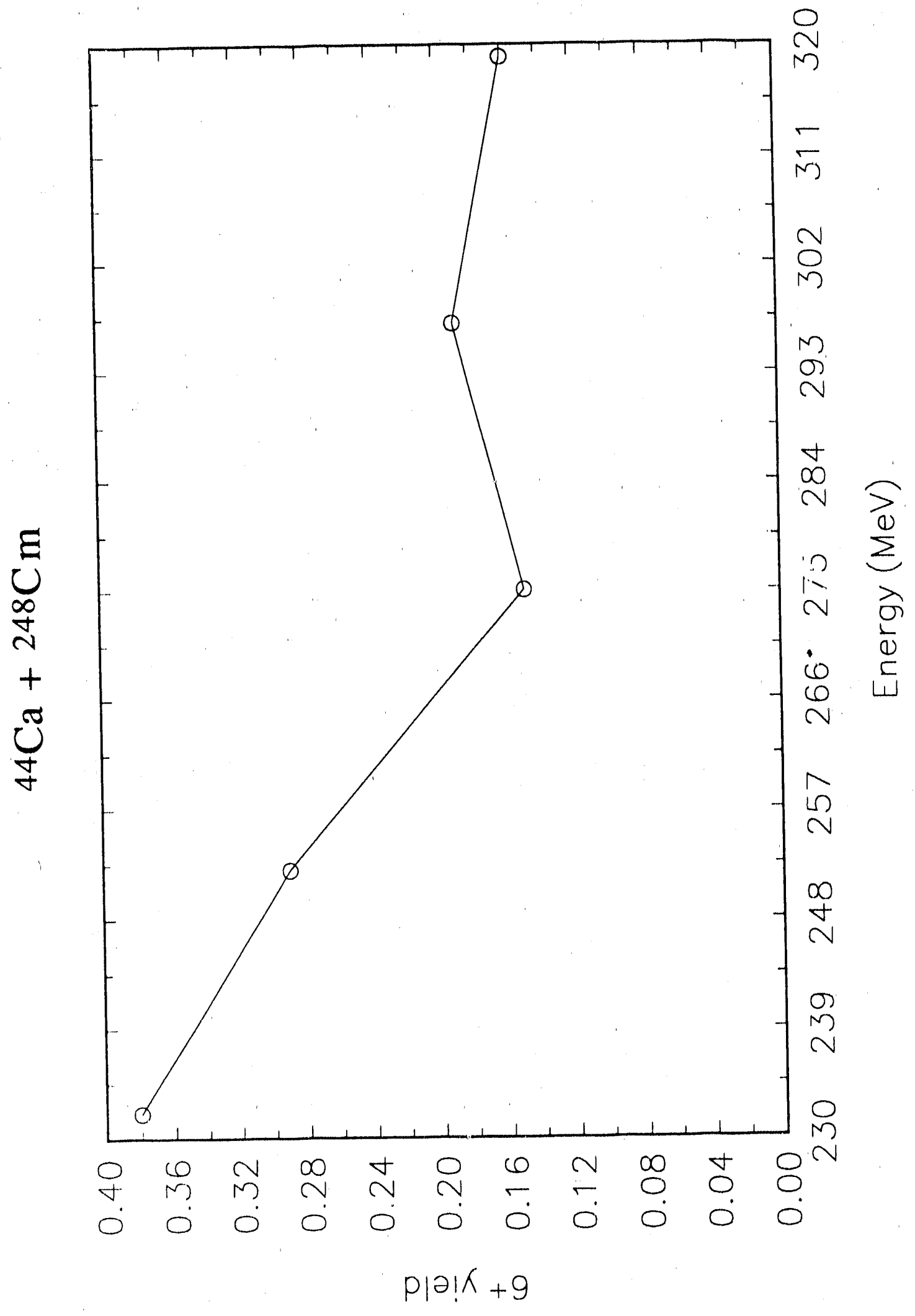

Fig. III.22 


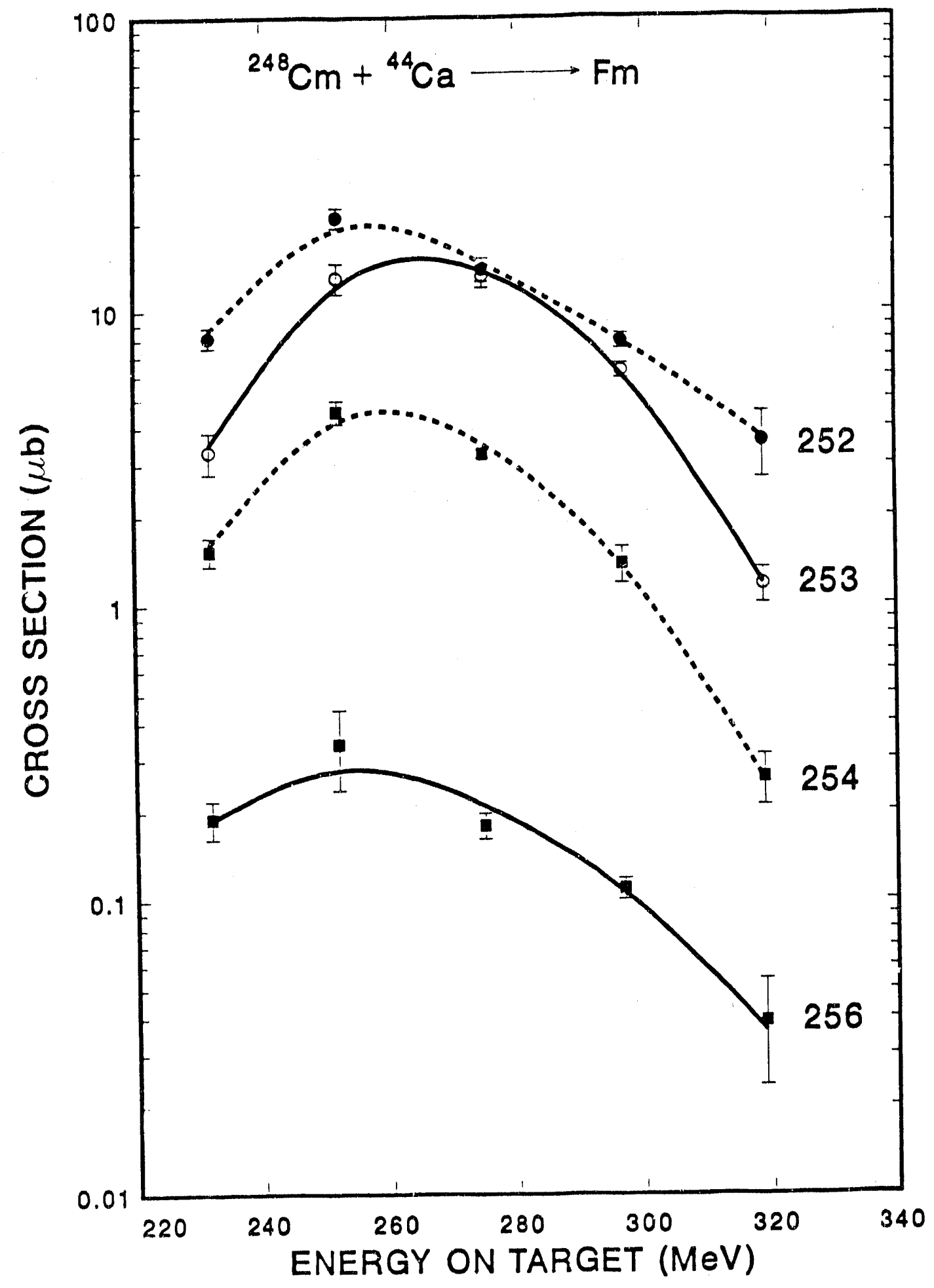

Fig. III.23 


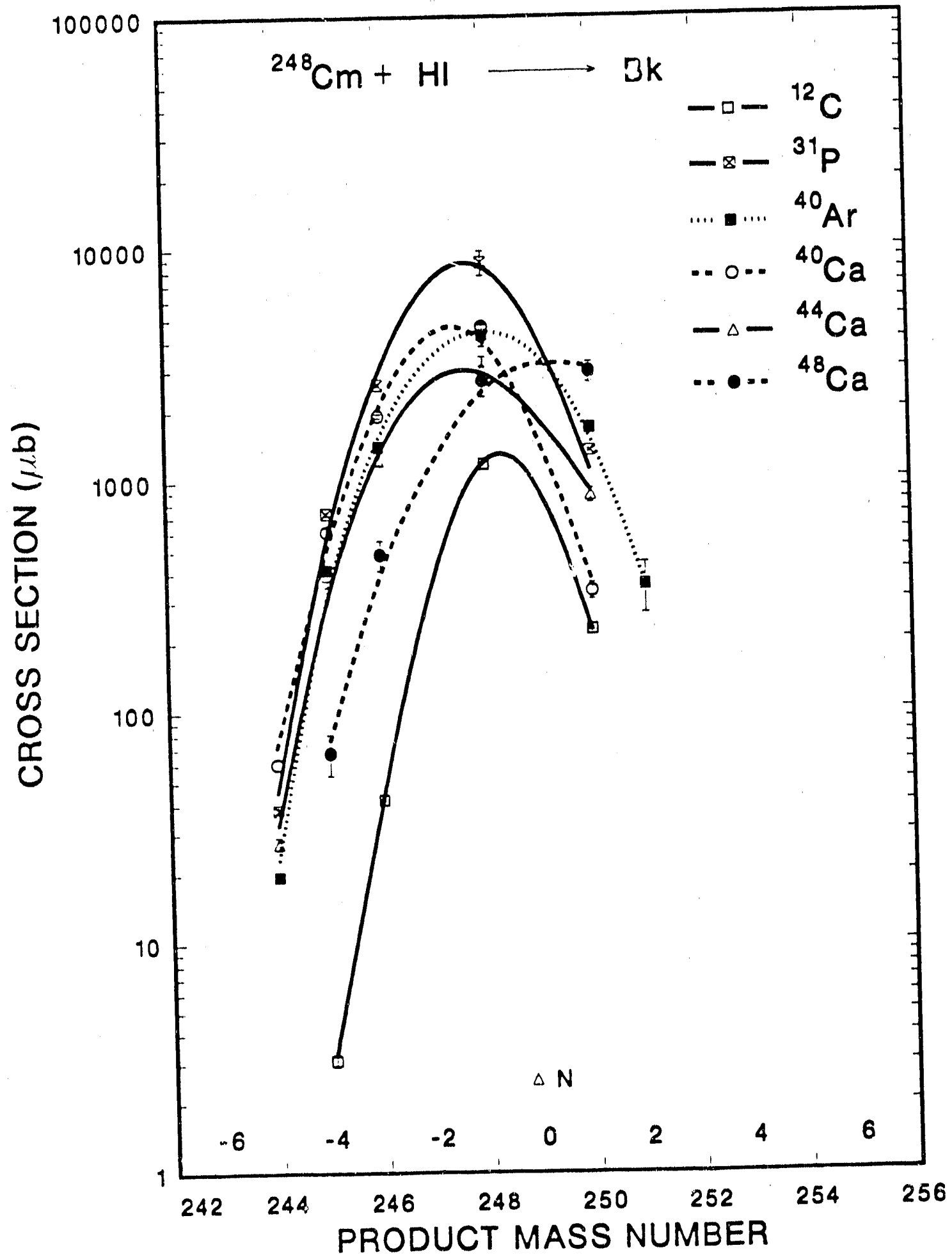

Fig. III.24 


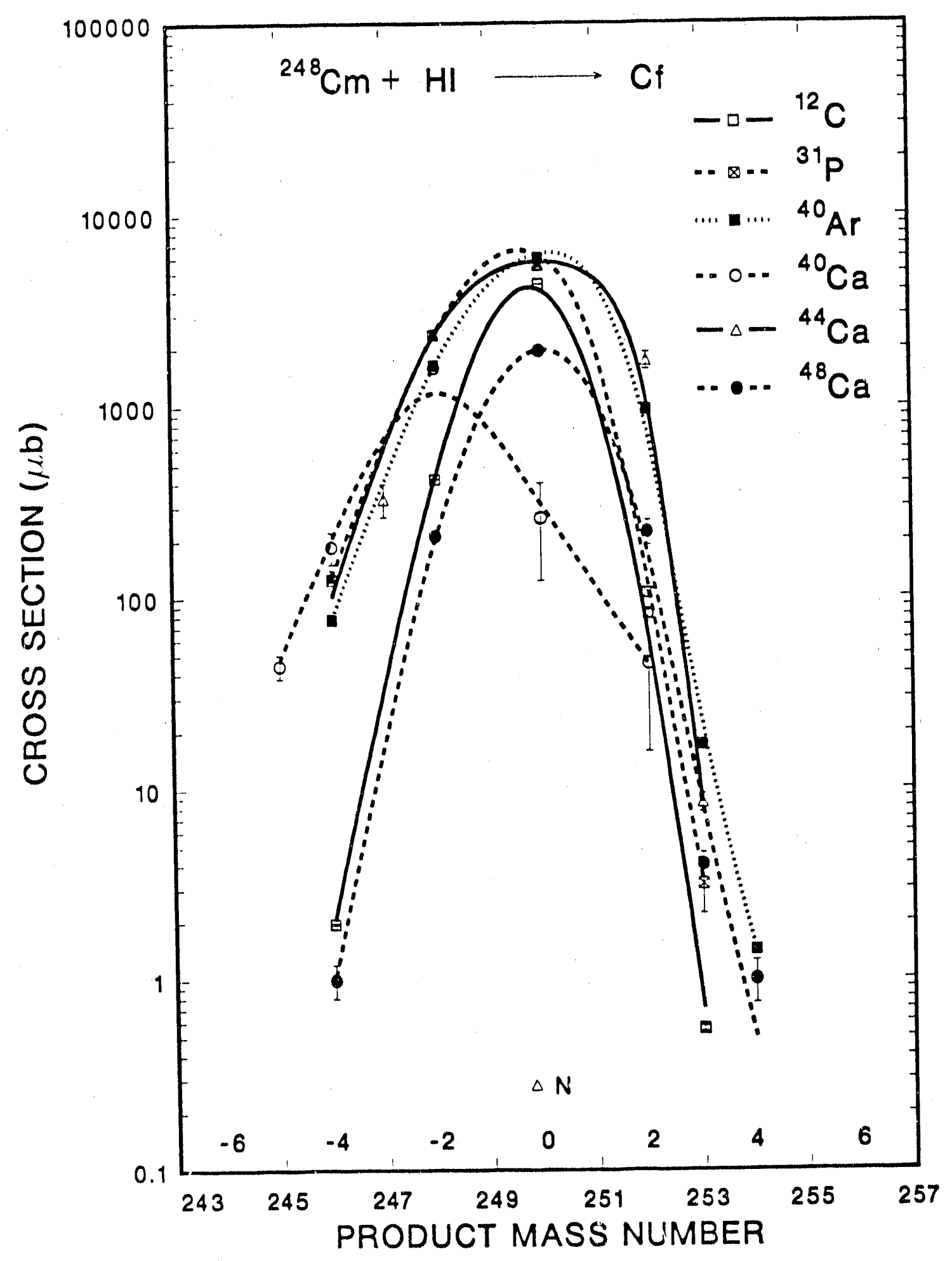

Fig. III.25 


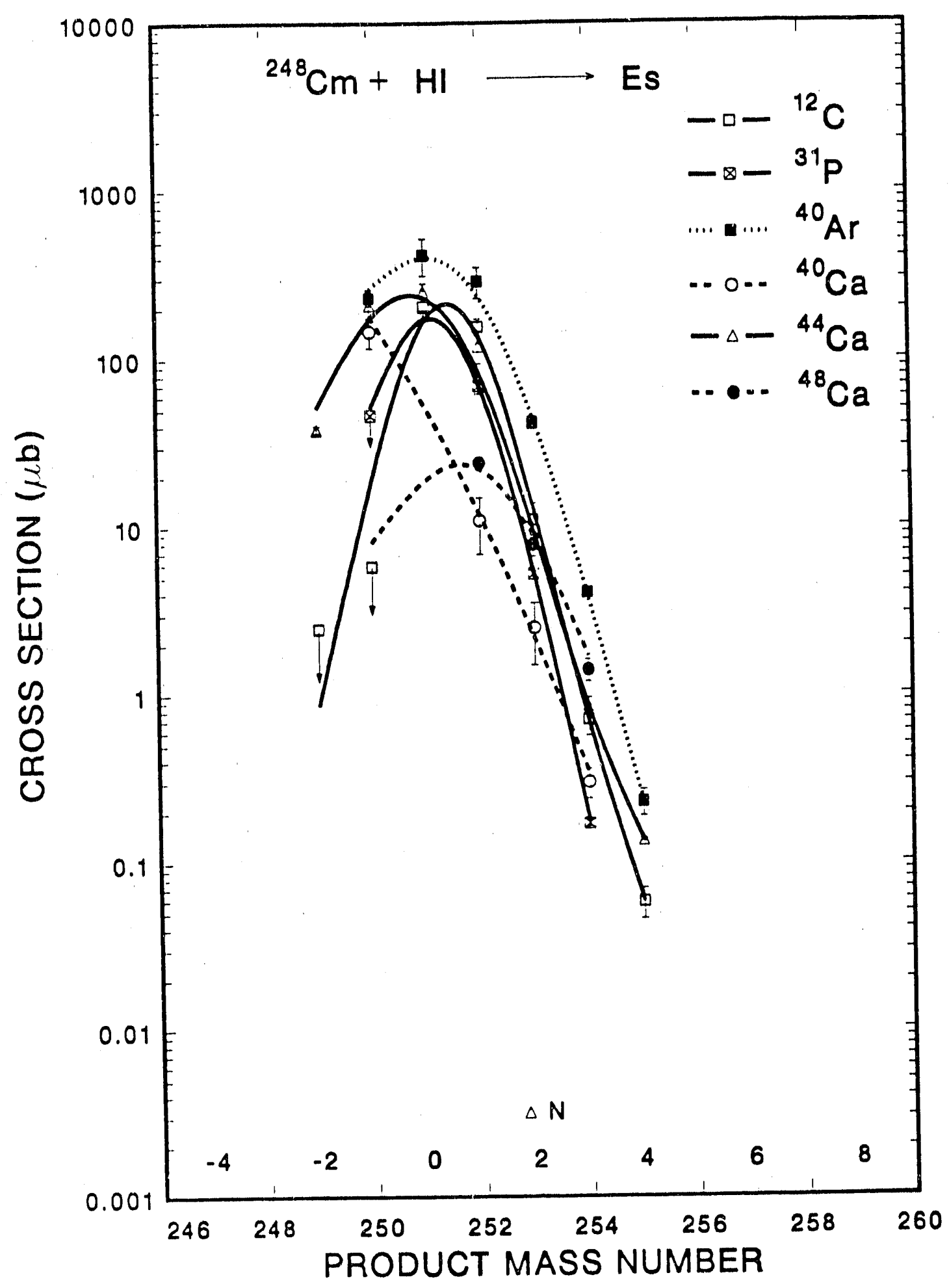

Fig. III.26 


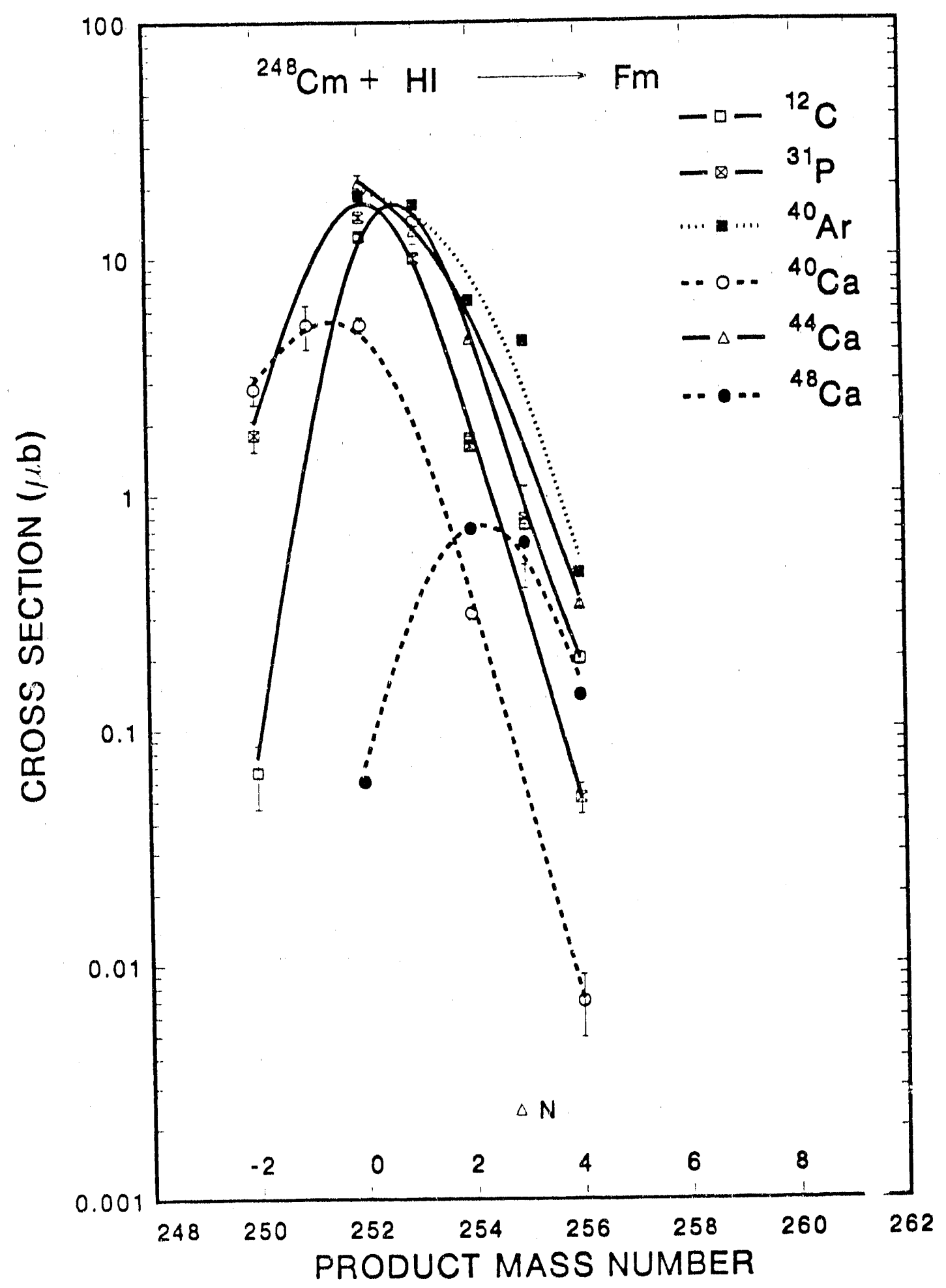

Fig. III.27 


\section{THEORY}

The theoretical treatment of transfer reactions is no trivial undertaking due to the extremely complex situations and the nonequilibrium conditions involved. However, several theories have been deveioped to model these processes [ABU80] [BIM72] [BR.172] [FRI83] [GAL69] [GAL70] [GRE85] [HAH74] [HAR83] [HOF88A] [KAU60] [NÖR75] [RAN86] [SIE71] [SIK72] [UDA82] [WIL80A]. These theoretical approaches are varied and range from simple semiclassical calculations to very intricate computations utilizing the distorted-wave Born approximation (DWBA). This list, however, is by no means complete and only a few of these theories will be discussed.

\section{A. Potential Energy Surfaces}

Potential energy surfaces (PES) have proven to be useful, especially in deeply inelastic reactions, for predicting the primary mass distributions of reaction products [TÜR89] [WEL85]. The most probable mass distribution should coincide with the highest level density in the dinuclear complex (composed of the projectile-like fragment and the target-like fragment), corresponding to a minimum in the potential energy of the system [GRO81]. $V_{\text {prod }}$, the potential energy of a specific product pair at an interaction radius, $R_{\text {int }}$ is

$$
V_{p r o d}-M_{t l}-M_{p l}=V_{C o u l}+V_{n u c l}+V_{c e n t}
$$

where $M_{t l}$ and $M_{p l}$ are the ground-state masses of the target-like and projectile-like fragments respectively, $V_{C o u l}$ is the Coulomb potential, $V_{n u c l}$ is the nuclear proximity potential [BLO77], and $V_{c e n t}$ is the centrifugal potential. The potential energy, PE, of a specific point on the PES is then defined as

$$
P E=V_{\text {prod }}-V_{\text {react }}-Q_{g g}
$$


where $V_{\text {react }}$ is the potential energy of the reactants and $Q_{g g}$ is the ground state $Q$ value. Thus, the $P E S$ is constructed from these $P E$ points.

Türler has calculated PES for the $40,44,48 \mathrm{Ca}-248 \mathrm{Cm}$ systems at $\cong 1.1 \times$ Coulomb barrier, with a corresponding grazing angular momentum of about 70 h [TÜR89]. Contour maps of these three systems are shown in Fig. IV.1. The equipotential energy contour lines are $4 \mathrm{MeV}$ apart, with the dashed lines representing $0 \mathrm{MeV}$. The dotted lines connect the calculated minimum potential energy points for each $Z$; hence, defining the valley of $N / Z$ equilibration. The position of ${ }^{248} \mathrm{Cm}$ (the injection point) is depicted by a cross $(+)$. Experimental maxima of the isotopic distributions are represented by filled circles and open circles are estimates of the maxima of unmeasured distributions.

In the ${ }^{40} \mathrm{Ca}$ system, the transfer of about four protons from the projectile to the target is required to reach $N / Z$ equilibration for the above target products. The position of ${ }^{248} \mathrm{Cm}$ in this system lies on a very "steep" wall of the PES on the neutron-rich side. Additionally, the experimentally determined maxima of the isotopic distributions all lie on the neutron-rich side of the $N / Z$ equilibration valley.

Like the ${ }^{40} \mathrm{Ca}$ system, the ${ }^{44} \mathrm{Ca}$ system has its injection point also displaced to the neutron-rich side of the valley of $N / Z$ equilibration by about four mass units. The experimentally determined maxima of the isotopic distributions lie on the neutron-rich side of the $N / Z$ equilibration valley, except for those products resulting from reactions involving the exchange of large numbers of nucleons such as Th and Fm. It should be noted that the maxima of the isotopic distributions for the above target products lie on or near the $N=152$ neutron subshell. Hence, this closed shell probably has a measurable effect on the final product distribution.

The ${ }^{48} \mathrm{Ca}$ system differs significantly from the ${ }^{40} \mathrm{Ca}$ and ${ }^{44} \mathrm{Ca}$ systems since the injection point is located on the line of $N / Z$ equilibration. All of the experimental maxima of the isotopic distributions for $Z=92-100$ lie on or very near the $N / Z$ 
equilibration line, indicating low initial excitation energy. Gäggeler [GÄG86] attributed the displacement of the maxima of the isotopic distributions of those products with $Z \leq 90$ to neutron evaporation from the excited primary fragments. The fact that the experimental maxima of the isotopic distributions for the ${ }^{40} \mathrm{Ca}$ and ${ }^{44} \mathrm{Ca}$ systems lie on the neutron-rich side of the valley of $N / Z$ equilibration indicates that the reaction products, particularly the above target products with $Z_{\text {prod }} \cong Z_{\text {target }}$, have essentially no excitation energy [TÜR89].

The PES have been calculated for the ${ }^{12} \mathrm{C},{ }^{31} \mathrm{P}$, and ${ }^{40} \mathrm{Ar}$ systems and contour maps are shown in Figs. IV.2-IV.4. The masses were calculated using the Myers and Swiatecki liquid droplet model [MYE77] [MYE80]. The odd-even term in the mass formula was neglected. Shell effects were included in these calculations while angular momentum effects were ignored. The equipotential lines have a spacing of $5 \mathrm{MeV}$. The minimum potential of each $Z$ is represented by an open circle. These circles have been connected by a dotted line to aid in viewing the contour plot. The solid circles represent the experimental maxima of the isotopic distributions, and the solid square represents the injection point, ${ }^{248} \mathrm{Cm}$.

The injection point for the ${ }^{12} \mathrm{C}$ system (see Fig. IV-2) lies on the neutron-rich side of the $N / Z$ equilibration line. However, the experimental maxima of the isotopic distributions lie on the neutron-deficient side of the $N / Z$ equilibration line, possibly due to neutron evaporation from excited primary fragments. Türler found the $18 \mathrm{O}$ system to behave similarly, with the experimental maxima of the isotopic distributions also being located on the neutron-deficient side of the N/Z equilibration line [TÜR89].

Fig. IV. 3 shows the PES contour plot for the ${ }^{31} \mathrm{P}$ system. The experimental maxima of the $\mathrm{Fm}$ and Es isotopic distributions lie on the $N / Z$ equilibration line, whereas the maxima of the $\mathrm{Bk}$ and $\mathrm{Cf}$ isotopic distributions lie on the neutron-rich side. Like the ${ }^{44} \mathrm{Ca}$ system, the maxima of the isotopic distributions for $Z=97-100$ from the ${ }^{31} \mathrm{P}$ system lie on or near the $N=152$ neutron subshell. 
The PES contour plot of the ${ }^{40} \mathrm{Ar}$ system is shown in Fig. IV.4. As with the $31 \mathrm{P}$ system, the experimental maxima of the $\mathrm{Bk}$ and $\mathrm{Cf}$ isotopic distributions lie on the neutron-rich side of the $N / Z$ equilibration line. However, the maxima of the $\mathrm{Fm}$ and $\mathrm{Es}$ curves lie on the neutron-deficient side of the $N / Z$ equilibration line, possibly indicating the formation of hot primary fragments and subsequent neutron evaporation.

As can be seen from Figs IV.1-IV-4, the PES do a relatively poor job of calculating the primary mass distributions of the above target reaction products $\left(Z_{\text {product }} \cong\right.$ $Z_{\text {target }}$ ) for the systems studied. $N / Z$ equilibration is not reached in many instances, especially in reactions involving the transfer of 1-2 protons and various numbers of neutrons. However, $N / Z$ equilibration is eventually reached after larger numbers of nucleons are exchanged between the target and projectile.

Additionally, since the peaks of the isotopic distributions, in many instances, lie on the neutron-rich side of the $N / Z$ equilibration line, the primary reaction products are most likely being formed with a small amount of excitation energy. This, however, does not hold for the ${ }^{12} \mathrm{C}$ or ${ }^{18} \mathrm{O}$ systems which both display maxima of isotopic distributions that are located on the neutron-deficient side of the $N / Z$ equilibration line.

\section{B. Two-Step Transfer Model}

As mentioned in Section I.B, Hahn et al. [HAH74] performed a detailed comparison between the experimental results of the ${ }^{239} \mathrm{Pu}\left({ }^{12} \mathrm{C}, \alpha 2-\alpha 3 n\right)$ reaction channels and the calculated values from a simple two-step kinematic model. Hahn et al. used the general expression obtained by Siemens et al. [SIE71] to treat only those cases where nucleons are transferred from the projectile to the target. In the first step of this semiclassical model, a cluster of nucleons is transferred from the projectile to the target. The transfer is assumed to occur at the nuclear surface and the velocity of the cluster is taken to be the same as the velocity of the projectile. Step two involves the Rutherford scattering of the light and heavy fragments formed in the transfer reaction. The model, however, does not treat the details of the separation of the projectile into the transferred aggregate and the 
residual product, nor does it consider the probability of a specific transfer process occurring. Additionally, the reaction $Q$ value is not treated in the calculation.

Specifically then, the final velocity, $V_{R f}$, at angle $\theta_{R}$ of the target-like fragment resulting from a transfer reaction is from the following two processes (see Fig. IV.5):

1. A group of nucleons is transferred from the projectile to the target, resulting in a recoiling nucleus $R$ with velocity $V_{R l}$.

2. : erford scattering between $R$ and the remaining light nucleus results in velocity $V_{R 2}$ at angle $\phi$.

For a specific reaction channel

$$
P+T-(T+A)+L=R+L
$$

where $P$ is the projectile, $T$ is the target, $A$ is the transferred aggregate, $L$ is the light residue, and $R$ is the recoiling nucleus. Hence, for step 1

$$
V_{R I}=\frac{M_{A}}{M_{R}} V_{P},
$$

where $M_{A}$ and $M_{R}$ are the masses of the transferred aggregate and recoiling heavy product nucle,us respectively, and $V_{P}$ is the velocity of the projectile.

Then, for step 2

$$
V_{R 2}=\frac{2 M_{L}}{M_{R}+M_{L}} V_{P} \cos \phi
$$

where $M_{L}$ is the mass of the light residue, and 


$$
\cos \phi=\frac{V_{c}}{2 E_{O}-V_{C}},
$$

where $V_{c}$ is the Coulomb barrier between $R$ and $L$ at the distance of closest approach, and $E_{O}$ is the energy in the center-of-mass system given by

$$
E_{o}=E_{P} \frac{M_{L} M_{R}}{M_{P}\left(M_{R}+M_{L}\right)},
$$

where $E_{P}$ is the kinetic energy of the projectile.

The calculated kinetic energy of the target-like fragment, $E_{R}$, and the emission angle of the target-like fragment, $\theta_{R}$, are compared with the experimental results obtained by Hahn et al. in Fig. IV.6A. The curves calculated for various reaction channels are labeled with the lighter fragment of that specific reaction channel (example: $\left.{ }^{239} \mathrm{Pu}\left({ }^{12} \mathrm{C},{ }^{3} \mathrm{He}\right){ }^{248} \mathrm{Cf}\right)$. As can be seen from the figure, the model does a fair job of reproducing the experimental results. Specifically, it reproduces the rapid decrease in $\theta_{R}$ with decreasing projectile bombarding energy.

Fig. IV.6B illustrates the results of a model developed by Galin et al. [GAL69] [GAL70] in which the incoming and outgoing trajectories are expressed only in terms of Rutherford scattering. This model requires the transfer to occur at the point of closest approach, $R_{\min }$, where the incoming $(i)$ and outgoing $(f)$ trajectories meet. Therefore

$$
R_{\text {min }}=\frac{Z_{P} Z_{T} e^{2}}{2 E_{i}}\left[1+\csc \left(1 / 2 \theta_{i}\right)\right]
$$

and 


$$
\mathrm{R}_{\min }=\frac{Z_{L} Z_{R} e^{2}}{2 E_{f}}\left[1+\csc \left(1 / 2 \theta_{f}\right)\right]
$$

where $E$ is the energy available in the center-of-mass system, and

$$
E_{f}=E_{i}-Q
$$

The sum $1 / 2 \theta_{i}+1 / 2 \theta_{f}$ is then taken to be the effective angle at which the light product is observed.

For Hahn's calculations, a distance of $14 \mathrm{fm}$ corresponding to $r_{O}=1.65 \mathrm{fm}$ was used to match the incoming and outgoing trajectories. The $Q$ values were chosen such that in each reaction sufficient excitation energy was available to evaporate neutrons from the $\mathrm{Cf}$ transfer reaction products to reach ${ }^{245} \mathrm{Cf}$ or ${ }^{244} \mathrm{Cf}$. Once again, the calculated curves reproduce the experimental data fairly well, consistent with the transfer of a "Be" cluster from ${ }^{12} \mathrm{C}$ to ${ }^{239} \mathrm{Pu}$. Hahn et al. point out the similarity of the calculated curves from the two different theories and caution against trying to interpret recoil data such as these in light of a specific transfer reaction model. However, Hahn et al. also note that these two theories do emphasize the importance of Coulombic scattering in transfer reactions.

\section{Gregorich's Model of Heavy Ion Damped Reactions}

Gregorich [GRE85] has developed a simple model for the theoretical determination of reaction cross sections of target-like products resulting from heavy ion bombardments of actinide targets. The model assumes a binary reaction mechanism in which the projectile collides with the target forming a dinuclear complex, which then exists for a time comparable to the nuclear transit time. During the lifetime of this dinuclear complex, nucleons, energy and angular momentum are exchanged. The model, however, ignores the exchange of angular momentum. Additionally, the charge distribution is assumed to be unequilibrated. An empirical description of the charge distribution as described by Wollersheim [WOL84] is used to calculate $\delta^{2} \sigma / \delta Z \delta E$. All of the kinetic energy loss is assumed to appear as excitation energy of the resulting dinuclear complex, 
and the equilibration of $N / Z$ is achieved by the transfer of veutrons only. The neutrons and excitation energy are statistically distributed between the two products according to a simple Fermi gas level density, yielding $\delta^{3} \sigma / \delta Z \delta N \delta E^{*}$, the triple differential cross section of the target-like products, where $E^{*}$ is the excitation energy. This distribution is then allowed to deexcite by the emission of neutrons in competition with fission to obtain the final cross section distribution. Charged particle emission was not considered.

Reaction cross sections based upon Gregorich's model were calculated using the computer code VMOD [GRE85] for target-like fragments for the ${ }^{31} \mathrm{P},{ }^{40} \mathrm{Ar}$, and ${ }^{44} \mathrm{Ca}$ systems at $\cong 1.16 \mathrm{X}$ Coulomb barrier and are shown in Figs. IV.7-IV.9. The experimental data points are connected with dashed lines while solid lines connect the calculated points.

For the ${ }^{31} \mathrm{P}$ system (Fig. IV.7), centroids of the calculated isotopic distributions of $\mathrm{Bk}, \mathrm{Cf}, \mathrm{Es}$, and Fm are too low when compared to the experimental centroids. The widths of the calculated $\mathrm{Cf}$ and Fm curves match the experimental widths fairly well. However, the width of the calculated Es distribution is much too wide. The calculated cross sections are typically too low, indicating that a significant fraction of the target-like reaction products are surviving neutron evaporation and fission. Hence, the reaction products are being formed with very little excitation energy. The model reproduces the Fm distribution better than the other distributions, although the calculated cross sections are lower than the experimental cross sections.

The calculated isotopic distributions for the ${ }^{40} \mathrm{Ar}$ system are shown in Fig. IV.8. The centroids for the $\mathrm{Bk}, \mathrm{Cf}$, and Es isotopic distributions are once again too low; however, the widths of the calculated distributions are fairly close to the widths of the experimental distributions. The calculated Es distributions reproduce the experimental distribution quite well, whereas the calculated Fm distribution is a bit high. The model does a very poor job of reproducing the $\mathrm{Bk}$ and $\mathrm{Cf}$ experimental distributions.

Fig. IV.9 illustrates the calculated and experimental isotopic distributions for the 
${ }^{44} \mathrm{Ca}$ system. As with the other two systems, the centroids of the calculated distributions are too low, with the possible exception of the Fm distribution. The experimental cross sections are mostly higher than the calcu'iated cross sections. The model does an excellent job of reproducing the Fm experimental isotopic distribution.

Cross sections, using Gregorich's model, were also calculated for the ${ }^{44} \mathrm{Ca}$. system at the Coulomb barrier. These results are plotted in Fig. IV.10. Like the ${ }^{44} \mathrm{Ca}$ system at $1.16 \mathrm{X}$ Coulomb barrier, the model typically estimates cross sections for $\mathrm{Bk}$ and $\mathrm{Cf}$ isotopes which are too low compared to the experimental cross sections. However, the calculated Fm cross sections are too large. The centroids of the calculated isotopic distributions are also low, with the exception of the Fm distribution.

Gregorich's model of damped reactions involving heavy ion projectiles and actinide targets inadequately reproduces the $\mathrm{Bk}, \mathrm{Cf}$, and Es experimental isotopic distributions from the ${ }^{31} \mathrm{P},{ }^{40} \mathrm{Ar}$, and ${ }^{44} \mathrm{Ca}$ systems. In contrast, the model satisfactorily duplicates the Fm experimental isotopic distributions from the three systems. In most of the cases studied, the experimental peak centroids of the isotopic distributions are on the neutronrich side of the calculated peak centroids, indicating that $N / Z$ equilibration is not being reached in these reactions. Also, cross sections calculated using Gregorich's model are consistently low for many of the above target actinide products, especially isotopes of Bk, Cf, and Es. This disagreement between Gregorich's model and the data indicate that these reaction products are being produced "cold"; therefore, surviving fission and/or neutron emission. Hence, Bk, Cf, Es, and perhaps some of the Fm isotopes produced in these reactions do not come from a damped reaction mechanism, but rather from a quasielastic type of reaction.

\section{Compound Nucleus Calculations: ALICE Code}

The computer code ALICE [BLA82] was used to calculate cross sections of above target reaction products from the ${ }^{12} \mathrm{C}_{-} 248 \mathrm{Cm}$ system based upon compound nucleus formation and subsequent particle evaporation. The code utilizes a standard Weisskopf- 
Ewing evaporation calculation [WEI40] with multiple particle emission including neutrons, protons, deuterons, and alphas. The code also includes the Bohr-Wheeler transition state model for fission [BOH39], and the hybrid/geometry dependent hybrid models for precompound decay [BLA71] [BLA72].

The results of the ALICE code calculation at 71 and $84 \mathrm{MeV}$ are plotted in Figs. IV.11 and IV.12 respectively, along with the cxperimental data at these energies. The Md cross sections at $71 \mathrm{MeV}$ are grossly overpredicted by ALICE which also incorrectly calculates the peak of the isotopic distribution $\omega$ be at mass number 257. At both energies, ALICE overnredicts the Fm cross sections by three orders of magnitude in some cases. The calculated peak Es cross section at $71 \mathrm{MeV}$ is at mass number 255 and has a value of 0.5 $\mu \mathrm{b}$. All other calculated Es cross sections at this energy are less than $0.01 \mu \mathrm{b}$. Hence, ALICE is underpredicting these Es cross section by several orders of magnitude. The calculated peak of the Es isotopic distribution at $84 \mathrm{MeV}$ is at mass number 253 versus the experimental maximum at 251 . At $84 \mathrm{MeV}$, ALICE does a better job of reproducing the Es experimental cross sections. However, at this energy, the calculated cross sections for the neutron-deficient Es isotopes are too low. Looking at the $\mathrm{Cf}$ isotopic distributions, ALICE predicts the peaks to be at mass numbers 252 and 251 for 71 and 84 $\mathrm{MeV}$ respectively, while the experimental maxima are at mass number 250 at both energies. In addition, the calculated $\mathrm{Cf}$ cross sections are too low at both energies. Finally, the calculated Bk cross sections are less than $0.01 \mu \mathrm{b}$ for all Bk isotopes at both energies, which is not in agreement with the experimental data. From these results one can conclude that ALICE does not do an adequate job of calculating the cross sections for

target-like fragments produced in the ${ }^{12} \mathrm{C}-248 \mathrm{Cm}$ system, thus indicating that a compound nucleus-type mechanism is not involved in the formation of these products.

\section{E. Partial Wave Calculations}

When a heavy ion projectile interacts with a target nucleus, the angular momentum, $L$, of the system is given by 


$$
L=p b \text {, }
$$

where $p$ is the linear momentum and $b$ is the impact parameter. Since the reduced de Broglie wavelength, $\mathcal{t}$, is

$$
\varkappa=\frac{\hbar}{p}
$$

then

$$
p=\frac{\hbar}{7}
$$

Substituting in for $p$ in Eq. (11), one gets

$$
L=\frac{b \hbar}{x} .
$$

The quantum mechanical restraints on the system require

$$
L=l \hbar
$$

where $l=0,1,2, \ldots \ldots$

Hence,

$$
b=1 X \text {. }
$$

Therefore, the cross section, $\sigma_{l}$, which corresponds to a collision having angular momentum th is given by

$$
\begin{aligned}
\sigma_{l} & =\pi \chi^{2}\left[(l+1)^{2}-l^{2}\right] T_{l} \\
& =\pi \star^{2}\left[l^{2}+2 l+1-l^{2}\right] T_{l} \\
& =\pi \chi^{2}[2 l+1] T_{l},
\end{aligned}
$$


where $T_{l}$ is the transmission coefficient. The total reaction cross section is then

$$
\begin{gathered}
\sigma_{r}=\sum_{l=0}^{\infty} \sigma_{l} \\
=\pi \lambda^{2} \sum_{l=0}^{\infty}(2 l+1) T_{l} .
\end{gathered}
$$

\section{Sikkeland's Theory of Compound Nucleus Formation}

Sikkeland et al. [SIK68] have calculated heavy-ion induced compound nucleus reaction cross sections using

$$
\sigma_{x}\left(E_{i}\right)=G^{x} \sigma_{c n} P_{x}
$$

where

$$
G=\left[\prod_{i=1}^{x}\left(\frac{\Gamma_{n}}{\Gamma_{n}+\Gamma_{f}}\right)_{i}\right]^{1 / x}
$$

and

$$
\begin{aligned}
& l_{c n} \\
& \sigma_{c n} P_{X}=\Sigma \sigma_{l}\left(E_{i}\right) P_{X, l}(E) \text {. } \\
& l=0
\end{aligned}
$$

$G$ is the mean value of $\Gamma_{n} /\left(\Gamma_{n}+\Gamma_{f}\right), \sigma_{c n}$ is the cross section for the formation of the compound nucleus, $P_{X}$ is the probability of emitting $x$ neutrons, $l_{c n}$ is the cutoff value where the reaction mechanism changes from compound nucleus reactions to grazing reactions, $\sigma_{l}\left(E_{i}\right)$ is the cross section for the lth partial wave at bombarding energy $E_{i}$, and 
$P_{x, l}(E)$ is the probability of emitting exactly $x$ neutrons from a compound nucleus of angular momentum $l$ and effective excitation energy $E$, based upon the Jackson model [JAK56]. Charged particle evaporation was ignored in these calculations.

\section{Sikkeland's Theory of Transfer Reactions}

Eq. (22) was modified by Sikkeland $e$ : al. [SIK72] to calculate cross sections for transfer reactions such as $\left({ }^{12} \mathrm{C}, \alpha \times n\right)$ in which a ${ }^{8} \mathrm{Be}$ is effectively transferred from the projectile to the target. Thus, the cross sections for transfer reactions are calculated from angular momentum values above the sharp cutoff value using

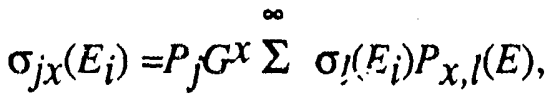

$$
\begin{aligned}
& l=l_{C n^{+1}}
\end{aligned}
$$

where $P_{j}$ is the mean probability of transferring exactly $j$ nucleons in an incomplete fusion process. Based upon existing experimental data, Sikkeland proposed an empirical method of calculating $P_{j}$,

$$
\log P_{j}=-\frac{1}{3}\left(0.1+Z_{j}\right)
$$

where $Z_{j}$ is the number of protons transferred to the target nucleus.

When comparing $\left({ }^{12} \mathrm{C}, \alpha x n\right)$ and $\left({ }^{13} \mathrm{C}, \alpha \times x n\right)$ reactions on ${ }^{249} \mathrm{Cf}$ (see Table IV.1), it was noted that the same value of $P_{j}$ did not fit both reaction channels well. The predicted energies for $x=1$ and 2 reactions agreed with the experimental data. However, for the $x=3$ and 4 reaction channels, the maximum predicted cross sections occurred at energies which were much too high. Additionally, for the $\left({ }^{12} \mathrm{C}, 2 \alpha 3 n\right)$ reaction, the predicted cross section value agreed fairly well with the experimental data. However, the predicted energy at which the maximum occurred was too large. 


\section{Wilczynski's Sum-Rule Model}

Wilczynski has developed a "Sum-Rule Model" to calculate absolute cross sections for complete and incomplete fusion reactions [WIL80A]. The absolute cross sections for each reaction channel can be determined from

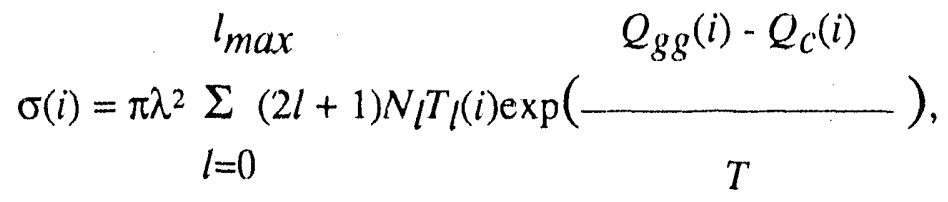

where $N_{l}$ is the normalization factor, $T_{l}$ is the transmission coefficient, $Q_{g g}$ is the ground state $Q$ value, $Q_{C}$ is the change in the Coulomb interaction energy due to charge transfer, and $l_{\max }$ is the largest $l$ value for which the total nucleus-nucleus potential is attractive and/or the distance of closest approach is smaller than the sum of the half-density radii.

The computer code SUMRUL, obtained from Oak Ridge [BEE87], was used to calculate the absolute cross sections for various exit channels for the ${ }^{12} \mathrm{C}-248 \mathrm{Cm}$ and ${ }^{40} \mathrm{Ar}-{ }^{248} \mathrm{Cm}$ systems. This code is based upon Wilczynski's "Sum-Rule Model." The results of the calculation along with some experimental results are plotted in Figs IV.13IV.16. The experimental cross sections are connected with dashed lines while the calculated cross sections are connected with solid lines. For the ${ }^{12} \mathrm{C}$ system, the model calculates peak cross sections for $\mathrm{Bk}$ and $\mathrm{Cf}$ which are too low by one or two orders of magnitude (see Fig IV.13). In addition, the model predicts that the peaks of the mass yield curves will be at mass numbers 249 and 252 for Bk and Cf, respectively, whereas the experimental peaks are at 248 and 250 , respectively. Fig IV.14 illustrates the calculated Es and $\mathrm{Fm}$ cross sections for the ${ }^{12} \mathrm{C}$ system. The model calculates cross sections for the heavier Es isotopes which are too high and misses the peak by two mass units. For the Fm cross sections, the model calculates the peak to be at mass number 256 while the experimental maximum is between 252 and 253 . The model also overestimates the neutron-rich Fm isotope cross sections by several orders of magnitude. 
The cross sections calculated using this model are fairly consistent with the experimental cross sections for the $\mathrm{Bk}$ and $\mathrm{Cf}$ isotopes produced in the interactions of ${ }^{40} \mathrm{Ar}$ with ${ }^{248} \mathrm{Cm}$ (Fig. IV.15). The calculated cross sections for the neutron-rich Cf isotopes are too high. The model fails badly on the neutron-rich Es and Fm isotopes as can be seen in Fig. IV.16. Not only are the calculated cross sections orders of magnitude too high, but the calculated peaks of the isotopic distributions are also wrong.

This model, however, does not include fission. If one assumes that the heavy fragment is emitted completely cold then the distributions in Figs. IV.13-IV.16 would effectively be the final isotopic distributions. If, however, the heavy fragment receives an amount of excitation energy which is comparable to its fission barrier and/or neutron binding energy, then the production cross section will be reduced. $\Gamma_{n} /\left(\Gamma_{n}+\Gamma_{f}\right)$ values for isotopes of Bk, Cf, Es and Fm, based on the empirical equations of Sikkeland et al. [SIK68] have been calculated and are given in Table IV.2. Including fission with the Bk and $\mathrm{Cf}$ cross section calculations for the ${ }^{12} \mathrm{C}$ system (Fig. IV.13) would cause greater disagreement with the experimental data. The calculated Es and Fm cross sections (Fig. IV.14) for the neutron-rich isotopes would have better agreement with the experimental cross sections. For the ${ }^{40} \mathrm{Ar}$ system, inclusion of fission would lower the cross sections of the neutron-rich $\mathrm{Cf}$ isotopes resulting in closer agreement between experimental and calculated cross sections. The calculated Es and Fm cross sections would be more consistent with the experimental data with the inclusion of fission.

\section{Presentation of New Model: PWA VE Calculations}

A simple method involving the calculation of geometric cross sections for binary reactions was developed by Hoffman and Hoffman [HOF88A]. This classical treatment involves the calculation of Rutherford trajectories for a given system. Specifically, given the $A, Z$, and energy of the projectile and the $A$ and $Z$ of the target, two parameters $R D$ and $C D$ are calculated. $R D$ is the difference between the radius of the projectile plus the target and the distance of closest approach of projectile and target for each Rutherford 
trajectory. $C D$ is the difference between the critical radius and the distance of closest approach for each Rutherford trajectory. If $R D>0$, an overlap between the two nuclei occurs and a binary reaction can take place. For $C D>0$, complete fusion between the target and projectile takes place. Hence, the impact parameters corresponding to $R D=0$ and $C D=0$ are calculated. The impact parameter area between these limits is then integrated to give the geometric cross section. This model assumes that the most probable number of nucleons exchanged between the target and projectile is related to the volume of the nuclear overlap region (i.e., the number of nucleons in the overlap region) and the exchange of nucleons is represented by a Gaussian distribution about this most probable value. The total binary reaction cross section for a given impact parameter is then distributed among twelve different mass numbers (one to twelve nucleons transferred) according to the Gaussian distribution. The impact parameter is incremented and the process is repeated over the interval $C D<0<R D$.

Cross sections based upon the model developed by Hoffman and Hoffman for the ${ }^{44} \mathrm{Ca}$ system at the Coulomb barrier are plotted in Figs. IV.17-IV.18. The calculated cross sections are connected by dotted lines while the experimental cross sections are connected by dashed lines. It should be noted that the calculated cross section for transferring $x$ nucleons is the total cross section of all such transfers involving $x$ nucleons. For example, there are sixteen possible ways to transfer four nucleons between two nuclei; therefore, these multiplicities must be taken into account when comparing the calculated cross sections with the experimental data for a particular reaction channel. The calculated isotopic distributions have widths that are approximately the same as the experimental distributions; however, the calculated cross sections for the neutrondeficient isotopes are typically too low.

Hoffman and Hoffman [HOF82] found that the reaction cross sections for above target nuclides produced in the $16,18 \mathrm{O}-248 \mathrm{Cm}$ systems were roughly proportional to $\exp \left(c E^{*}\right)$ where $c$ varies from 0.35 for $\mathrm{Fm}$ to 0.6 for Bk. Artukh et al. [ART71] found a 
similar relationship for the ${ }^{16} \mathrm{O}-232 \mathrm{Th}$ and ${ }^{16} \mathrm{O}-197 \mathrm{Au}$ systems. Based on these results, the cross sections calculated with the Hoffman model were multiplied by the proportionality constant $\exp \left(c E^{*}\right)$, where $c$ was set equal to 0.4 . These cross sections are connected with solid lines in Figs. IV.17 and IV.18. As can be seen from these figures, the calculated Bk and Es cross sections match the experimental values very well. The model does a reasonable job for the neutron-rich and neutron-deficient $\mathrm{Cf}$ isotopes, but the calculated cross sections for $249-252 \mathrm{Cf}$ are slightly smaller than the experimental cross sections. For $255-256 \mathrm{Fm}$, the model does an excellent job. However, it overestimates the cross sections of $252-254 \mathrm{Fm}$. The calculated peaks of the isotopic distributions are at ${ }^{247} \mathrm{Bk},{ }^{248} \mathrm{Cf},{ }^{251} \mathrm{Es}$, and $252 \mathrm{Fm}$, versus the experimental maxima at $248 \mathrm{Bk}, 250 \mathrm{Cf}, 250_{\mathrm{Es}}$, and $\mathrm{S}^{252} \mathrm{Fm}$.

Figs. IV.19-IV.21 illustrate the calculated cross sections from the Hoffman model using the proportionality $\exp \left(0.4 E^{*}\right)$ for the ${ }^{40} \mathrm{Ar},{ }^{31} \mathrm{P}$, and ${ }^{12} \mathrm{C}$ systems. The calculated cross sections are connected by solid lines while the experimental points are connected with dashed lines. The calculated $\mathrm{Bk}$ and $\mathrm{Fm}$ isotopic distributions from the $40 \mathrm{Ar}$ system (see Fig. IV.19) match the experimental distributions very well. Like the ${ }^{44} \mathrm{Ca}$ system, the calculated cross sections for the neutron-deficient and neutron-rich $\mathrm{Cf}$ isotopes fit the experimental values very well, however, the model once again calculates cross sections for $249-252 \mathrm{Cf}$ which are too low. The calculated cross sections for 253 ${ }^{254}$ Es accurately depict the experimental values but the calculated cross sections for the other measured Es isotopes are too low.

The calculated Bk and $\mathrm{Cf}$ isotopic distributions from the ${ }^{31} \mathrm{P}$ system (see Fig. IV.20) fit the experimental distributions reasonably well. However, the model overestimates the cross sections of all the measured Es and Fm isotopes. Additionally, the model correctly predicts the peaks of the Cf, Es, and Fm isotopic distributions to be at mass numbers 250, 251 and 252 respectively.

Discrepancies arise between the calculated cross sections and the experimental cross 
sections from the ${ }^{12} \mathrm{C}$ system (Fig. IV.21). The model underestimates the yields of the neutron-deficient $\mathrm{Cf}$ and $\mathrm{Es}$ isotopes. Since the $E_{X}$ values for the Fm and $\mathrm{Md}$ isotopes from the ${ }^{12} \mathrm{C}$ system increase drastically with $A$ (see Tables III.9 and III.10), the calculated cross sections also increase; hence, the model does a very poor job on the neutron- rich Fm and $\mathrm{Md}$ isotopes. However, the model does a fairly good job of calculating the $\mathrm{Bk}$ isotopic distribution even though the calculated cross sections are a little low.

Assuming that the excitation energy is partitioned according to the mass fraction of the projectile transferred to the target, the effective excitation energy, $E_{\text {eff, }}$ can be calculated using

$$
E_{e f f}=\frac{N}{A} E_{x},
$$

where $N$ is the total number of nucleons transferred between the target and projectile, $A$ is the mass number of the projectile, and $E_{X}$ is the excitation energy described earlier.

The results of this calculation at 236,275 and $319 \mathrm{MeV}$ for the ${ }^{44} \mathrm{Ca}$ system are given in Table IV.III. As can be seen, even at the highest bombarding energy studied, the effective excitation energy is less than $2.5 \mathrm{MeV}$ for the above target products; hence, these products are being formed essentially cold. The experimental data corroborates this conclusion since the cross sections for the various Bk, Cf, and Es isotopes measured do not appreciably decrease even at energies of $1.37 \times$ Coulomb barrier. Similar results were obtained for the ${ }^{31} \mathrm{P}$ and ${ }^{40} \mathrm{Ar}$ systems.

The effective excitation energies for the ${ }^{12} \mathrm{C}$ system are given in Table IV.IV. Since the mass number of ${ }^{12} \mathrm{C}$ is much smaller than ${ }^{44} \mathrm{Ca},{ }^{40} \mathrm{Ar}$, and ${ }^{31} \mathrm{P}$, the fraction of mass transferred from the projectile to the target is much greater; therefore, the effective excitation of the above target products is greater. The $E_{\text {eff }}$ 's of ${ }^{12} \mathrm{C}$ system for the $\mathrm{Bk}$ 
and $\mathrm{Cf}$ isotopes of interest are $\leq 4.7 \mathrm{MeV}$. However, for the Es and Fm isotopes, the $E_{\text {eff }}$ values begin to exceed the neutron binding energies and/or fission barriers. For these isotopes, one would thus expect depletion of the cross sections due to fission and/or neutron emission. 
Table IV.1. Experimental and calculated peak cross sections for No and Fm isotopes produced from the interactions of $12,13 \mathrm{C}$ with ${ }^{249} \mathrm{Cf}$. Taken from [ESK75].

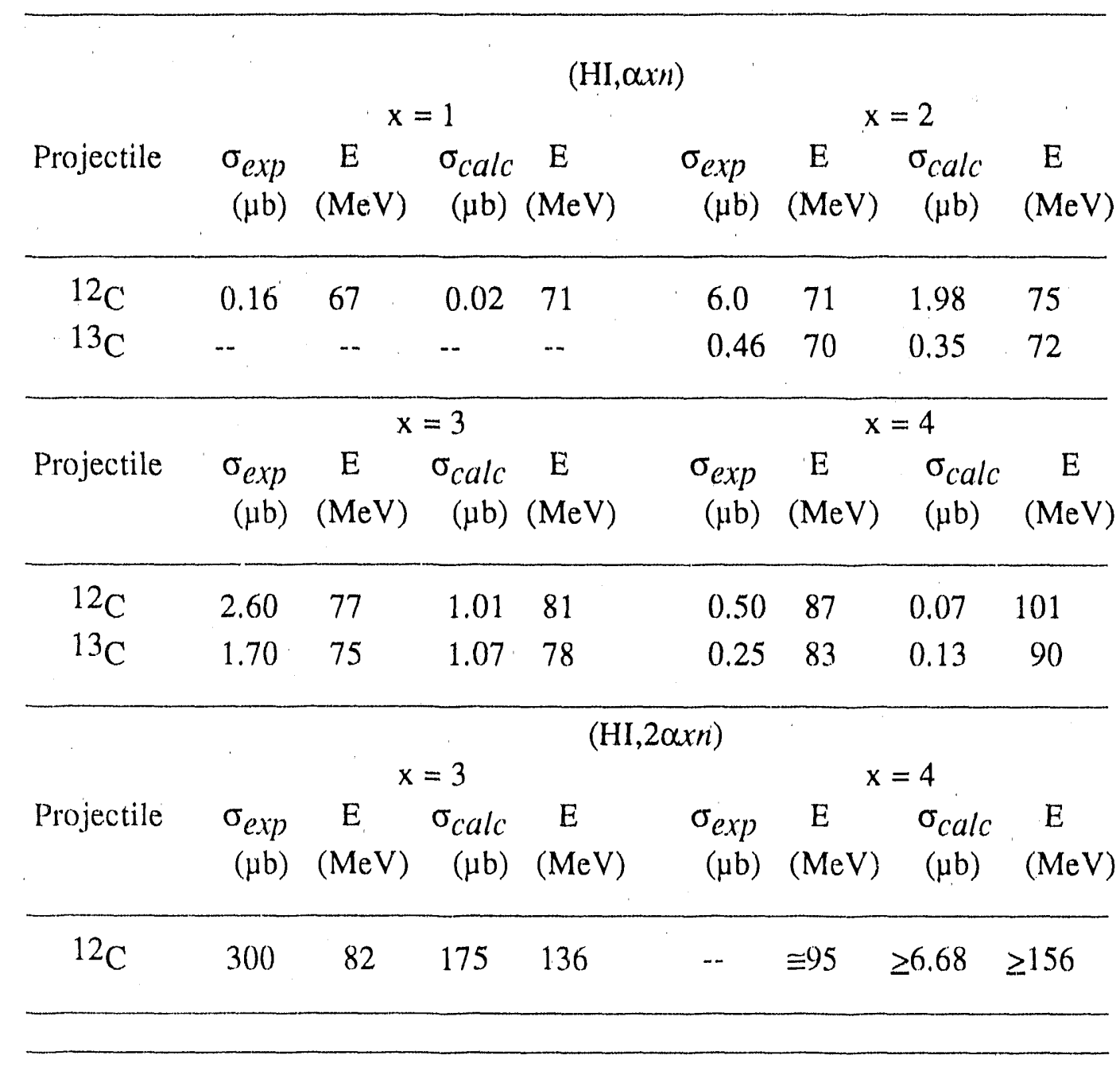


Table IV.2. Calculated $\Gamma_{n} /\left(\Gamma_{n}+\Gamma_{f}\right)$ values for various actinides based upon the empirical equations of Sikkeland et al. [SIK68].

\begin{tabular}{rrrr}
\hline & & & \\
\hline & & & \\
Nuclide & & Nuclide & $\Gamma_{n}$ \\
\cline { 2 - 4 } & $\Gamma_{n}+\Gamma_{f}$ & & $\Gamma_{n}+\Gamma_{f}$ \\
\hline Bk 244 & 0.20 & Es 249 & 0.15 \\
245 & 0.25 & 250 & 0.20 \\
246 & 0.32 & 251 & 0.26 \\
247 & 0.39 & 252 & 0.32 \\
248 & 0.47 & 253 & 0.35 \\
249 & 0.55 & 254 & 0.37 \\
250 & 0.63 & 255 & 0.40 \\
251 & 0.66 & 256 & 0.43 \\
& & & \\
Cf 246 & 0.12 & Fm 250 & 0.07 \\
247 & 0.16 & 251 & 0.09 \\
248 & 0.21 & 252 & 0.12 \\
249 & 0.26 & 253 & 0.16 \\
250 & 0.33 & 254 & 0.18 \\
251 & 0.41 & 255 & 0.19 \\
252 & 0.43 & 256 & 0.21 \\
253 & 0.46 & 254 & 0.49 \\
\hline
\end{tabular}


Table IV.3. Excitation energies, $E_{x}$, and effective excitation energies, $E_{\text {eff }}$, for above target actinide products of the ${ }^{44} \mathrm{Ca}-248 \mathrm{Cm}$ system at $236 \mathrm{MeV}$ (1.0 X Coulomb barrier), $275 \mathrm{Mev}$ (1.16 X Coulomb barrier) and $319 \mathrm{MeV}$ (1.35 X Coulomb barrier). $E_{\text {eff }}$ was calculated assuming $E_{X}$ is apportioned according to the fraction of projectile mass transferred between the target and projectile.

\begin{tabular}{|c|c|c|c|c|c|c|}
\hline \multirow{2}{*}{ Nuclide } & \multicolumn{2}{|c|}{$236 \mathrm{MeV}$} & \multicolumn{2}{|c|}{$275 \mathrm{MeV}$} & \multicolumn{2}{|c|}{$319 \mathrm{MeV}$} \\
\hline & $\begin{array}{c}E_{x} \\
(\mathrm{McV})\end{array}$ & $\begin{array}{l}E_{\text {eff }} \\
(\mathrm{MeV})\end{array}$ & $\begin{array}{l}E_{x} \\
(\mathrm{MeV})\end{array}$ & $\begin{array}{l}E_{\text {eff }} \\
(\mathrm{MeV})\end{array}$ & $\begin{array}{c}E_{x} \\
(\mathrm{MeV})\end{array}$ & $\begin{array}{l}E_{\text {eff }} \\
(\mathrm{McV})\end{array}$ \\
\hline Bk 244 & 6.8 & 0.9 & 3.6 & 0.5 & -0.1 & $\cdots$ \\
\hline 245 & 8.8 & 1.0 & 6.4 & 0.7 & 3.8 & 0.4 \\
\hline 246 & 5.5 & 0.5 & 4.0 & 0.4 & 2.2 & 0.2 \\
\hline 247 & 5.4 & 0.4 & 4.6 & 0.3 & 3.8 & 0.3 \\
\hline 248 & 1.4 & 0.1 & 1.4 & 0.1 & 1.4 & 0.1 \\
\hline 249 & 0.3 & 0.0 & 1.1 & 0.0 & 1.9 & 0.0 \\
\hline 250 & -4.7 & -.. & -3.3 & $\cdots$ & -1.6 & -.. \\
\hline 251 & -6.0 & $\cdots$ & -3.9 & $\cdots$ & -1.5 & $\cdots$ \\
\hline Cf 246 & 8.4 & 1.1 & 6.8 & 1.0 & 5.1 & 0.7 \\
\hline 247 & 5.9 & 0.7 & 5.2 & 0.6 & 4.3 & 0.5 \\
\hline 248 & 7.2 & 0.7 & 7.2 & 0.7 & 7.2 & 0.7 \\
\hline 249 & 4.1 & 0.3 & 4.8 & 0.3 & 5.6 & 0.4 \\
\hline 250 & 4.7 & 0.2 & 6.2 & 0.3 & 7.8 & 0.4 \\
\hline 251 & 0.1 & 0.0 & 2.2 & 0.2 & 4.6 & 0.3 \\
\hline 252 & -0.2 & $\ldots$ & 2.6 & 0.2 & 5.7 & 0.5 \\
\hline 253 & -5.7 & ... & -2.3 & $\ldots$ & 1.6 & 0.2 \\
\hline 254 & -6.6 & $\cdots$ & -2.6 & $\cdots$ & 2.0 & 0.3 \\
\hline Es 249 & 2.3 & 0.3 & 3.0 & 0.3 & 3.8 & 0.4 \\
\hline 250 & 1.2 & 0.1 & 2.6 & 0.2 & 4.3 & 0.4 \\
\hline 251 & 2.5 & 0.2 & 4.6 & 0.3 & 7.0 & 0.5 \\
\hline 252 & -0.5 & -.. & 2.3 & 0.2 & 5.5 & 0.5 \\
\hline 253 & -0.3 & -.. & 3.1 & 0.4 & 7.0 & 0.8 \\
\hline 2.54 & -3.6 & -.. & 0.3 & 0.0 & 4.9 & 0.7 \\
\hline 255 & -4.1 & ... & 0.5 & 0.1 & 5.7 & 0.9 \\
\hline 256 & -9.9 & -.. & -4.7 & -.- & 1.1 & 0.2 \\
\hline Fm 251 & 0.4 & 0.0 & 2.5 & 0.3 & 4.9 & 0.6 \\
\hline 252 & 3.4 & 0.3 & 6.1 & 0.6 & 9.3 & 0.8 \\
\hline 253 & 1.3 & 0.1 & 4.7 & 0.5 & 8.5 & 1.0 \\
\hline 254 & 3.2 & 0.4 & 7.2 & 1.0 & 11.8 & 1.6 \\
\hline 255 & 0.0 & 0.0 & 4.6 & 0.7 & 9.8 & 1.6 \\
\hline 256 & 1.7 & 0.3 & 6.9 & 1.3 & 12.7 & 2.3 \\
\hline
\end{tabular}


Table IV.4. Excitation energies, $E_{x}$, and effective excitation energies, $E_{\text {eff }}$, for above target actinide products of the ${ }^{12} \mathrm{C}-248 \mathrm{Cm}$ system at $72 \mathrm{MeV}$ (1.0 X Coulomb barrier), $84 \mathrm{Mev}$ (1.16 X Coulomb barrier) and $97 \mathrm{MeV}$ (1.35 X Coulomb barrier). $E_{\text {eff }}$ was calculated assuming $E_{X}$ is apportioned according to the fraction of projectile mass transferred between the target and projectile.

\begin{tabular}{|c|c|c|c|c|c|c|}
\hline \multirow[b]{2}{*}{ Nuclide } & \multicolumn{2}{|c|}{$72 \mathrm{MeV}$} & \multicolumn{2}{|c|}{$84 \mathrm{MeV}$} & \multicolumn{2}{|c|}{$97 \mathrm{MeV}$} \\
\hline & $\begin{array}{c}E_{x} \\
(\mathrm{MeV})\end{array}$ & $\begin{array}{l}E_{\text {eff }} \\
(\mathrm{MeV})\end{array}$ & $\begin{array}{c}E_{x} \\
(\mathrm{MeV})\end{array}$ & $\begin{array}{l}E_{\text {eff }} \\
(\mathrm{MeV})\end{array}$ & $\begin{array}{c}E_{x} \\
(\mathrm{MeV})\end{array}$ & $\begin{array}{c}E_{\text {eff }} \\
(\mathrm{MeV})\end{array}$ \\
\hline Bk 244 & -19.2 & $\ldots-$ & -23.3 & $\ldots$ & -27.7 & $\ldots$ \\
\hline 245 & -12.2 & -.. & -15.2 & $\ldots$ & -18.4 & -.. \\
\hline 246 & -9.3 & $\ldots$ & -11.2 & $\cdots$ & -13.4 & -.. \\
\hline 247 & -3.5 & ... & -4.4 & $\ldots$ & -5.5 & ... \\
\hline 248 & -3.5 & -.. & -3.5 & $\ldots$ & -3.5 & -.. \\
\hline 249 & -0.7 & -.. & 0.3 & 0.0 & 1.3 & 0.1 \\
\hline 250 & -7.6 & -.. & -5.8 & -.. & -3.8 & $\ldots$ \\
\hline 251 & -9.9 & $\cdots$ & -7.2 & $\ldots$ & -4.3 & ... \\
\hline Cf 246 & -15.3 & '-.- & -17.2 & $\ldots$ & -19.4 & $\ldots$ \\
\hline 247 & -11.6 & $\ldots$ & -12.6 & $\ldots$ & -13.6 & $-\cdots$ \\
\hline 248 & -3.0 & $\ldots$ & -3.0 & $\ldots$ & $-3,0$ & -.. \\
\hline 249 & -0.9 & ... & 0.0 & 0.0 & 1.0 & 0.3 \\
\hline 250 & 4.8 & 0.8 & 6.7 & 1.1 & 8.7 & 1.4 \\
\hline 251 & 2.8 & 0.7 & 5.5 & 1.4 & 8.4 & 2.1 \\
\hline 252 & 6.9 & 2.3 & 10.4 & 3.5 & 14.2 & 4.7 \\
\hline 253 & -7.6 & ... & -3.4 & $\ldots$ & 1.3 & 0.5 \\
\hline 254 & -12.8 & $\ldots$ & -7.8 & -- & -2.3 & -.. \\
\hline Es 249 & -11.7 & $\ldots$ & -10.8 & $\ldots$ & -9.8 & $\ldots$ \\
\hline 250 & -6.9 & -.. & -5.1 & -.- & -3.1 & -.. \\
\hline 251 & 0.4 & 0.1 & 3.1 & 0.8 & 6.0 & 1.5 \\
\hline 252 & 1.3 & 0.5 & 4.8 & 1.6 & 8.6 & 2.9 \\
\hline 253 & 5.3 & 2.2 & 9.6 & 4.0 & 14.2 & 5.9 \\
\hline 254 & 2.7 & 1.4 & 7.7 & 3.9 & 13.2 & 6.6 \\
\hline 255 & 2.7 & 1.6 & .8 .4 & 4.9 & 14.6 & 8.5 \\
\hline 256 & -14.4 & -.. & -8.0 & -.. & -1.1 & -.. \\
\hline $\mathrm{Fm} 252$ & 3.0 & 1.0 & 6.5 & 2.2 & 10.3 & 3.4 \\
\hline 253 & 5.7 & 2.4 & 10.0 & 4.2 & 14.7 & 6.1 \\
\hline 254 & 12.5 & 6.2 & 17.5 & 8.7 & 22.9 & 11.5 \\
\hline 255 & 15.5 & 9.0 & 21.2 & 12.4 & 27.4 & 16.0 \\
\hline 256 & 22.4 & 14.9 & 28.8 & 19.2 & 35.7 & 23.8 \\
\hline Md 255 & 4.5 & 2.6 & 10.2 & 6.0 & 16.4 & 9.6 \\
\hline 256 & 9.6 & 6.4 & 16.0 & 10.7 & 23.0 & 15.3 \\
\hline 257 & 18.8 & 14.1 & 25.9 & 19.4 & 33.5 & 25.1 \\
\hline 2.58 & 17.6 & 14.6 & 25.2 & 21.0 & 33.5 & 27.9 \\
\hline
\end{tabular}




\section{Figure Captions}

Figure IV.1. Potential energy surface contour plots for the $40,44,48 \mathrm{Ca}-248 \mathrm{Cm}$ systems at a center-of-mass energy of $1.1 \times$ Coulomb barrier and $l=50 \hbar$. The equipotential energy contour lines have a spacing of $4 \mathrm{MeV}$. The dashed lines represent $0 \mathrm{MeV}$. The position of the ${ }^{248} \mathrm{Cm}$ target (called the injection point) is signified with a cross $(+)$. The dotted lines connect the calculated minimum potential energy points of each $Z$. The filled circles indicate the experimentally determined maxima of the isotopic distributions and the open circles are the estimated maxima of isotopic distributions based upon experimental data where maxima where not seen. Figure taken from [TÜR89].

Figure IV.2. Potential energy surface contour plot of the ${ }^{12} \mathrm{C}$ system at $0 \hbar$. The open circles represent the minimum calculated potential energy of each $Z$ and are connected by a dotted line. The solid circles represent the experimentally determined maxima of the isotopic distributions. The ${ }^{248} \mathrm{Cm}$ injection point is represented by a filled square.

Figure IV.3. Potential energy surface contour plot of the $31 \mathrm{P}$ system at $0 \hbar$. The open circles represent the minimum calculated potential energy of each $Z$ and are connected by a dotted line. The solid circles represent the experimentally determined maxima of the isotopic distributions. The ${ }^{248} \mathrm{Cm}$ injection point is represented by a filled square.

Figure IV.4. Potential energy surface contour plot of the ${ }^{40} \mathrm{Ar}$ system at $0 \hbar$. The open circles represent the minimum calculated potential energy of each $Z$ and are connected by a dotted line. The solid circles represent the experimentally determined maxima of the isotopic distributions. The ${ }^{248} \mathrm{Cm}$ injection point is represented by a filled square.

Figure IV.5. Laboratory system vector diagram for Two-Step Transfer Model. 'Recoiling target-like fragment $R$ with mass $M_{R}$ has a velocity $V_{R j}$ at angle $\theta_{R}$ which is 
the resultant of velocity $V_{R I}$ at $0^{\circ}$, due to the transfer of an aggregate of nucleons from the projectile to the target, and of velocity $V_{R 2}$ at angle $\phi$, due to Rutherford scattering with the remaining projectile-like product $L$, with mass $M_{L}$. Figure taken from [HAH74].

Figure IV.6. Experimentally determined recoil energies, $E_{R}$, and scattering angle of the heavy recoiling reaction products using the Two-Step Transfer Model of Hahn [HAH74]. These results were derived from range and angular distributions measured for ${ }^{245} \mathrm{Cf}$ and ${ }^{244} \mathrm{Cf}$. The circles represent the centroids of the distributions while the triangles represent the maxima of the angular distributions. The curved lines in part (a) are the results of a calculation involving the Two-Step Transfer Model, while those lines in part (b) are the results of a calculation which has considered only Rutherford scattering. Eacn curve is identified by the He isotope remaining after the transfer reaction. The solid lines represent those cases in which sufficient excitation energy is available in the intermediate nucleus to evaporate neutrons to reach ${ }^{244} \mathrm{Cf}$, while the dashed curves represent those cases with sufficient excitation energy to reach $245 \mathrm{Cf}$. Figure taken from [HAH74].

Figure IV.7. Calculated isotopic distributions (symbols connected by solid lines) for $\mathrm{Bk}, \mathrm{Cf}, \mathrm{Es}$, and $\mathrm{Fm}$ from the interactions of $207 \mathrm{MeV}\left(1.17 \mathrm{X}\right.$ Coulomb barrier) ${ }^{31} \mathrm{P}$ ions with ${ }^{248} \mathrm{Cm}$. These calculations are based upon Gregorich's model of heavy ion damped reactions. The experimentally determined isotopic distributions are represented by the symbols connected by dashed lines.

Figure IV.8. Calculated isotopic distributions (symbols connected by solid liries) for $\mathrm{Bk}, \mathrm{Cf}, \mathrm{Es}$, and $\mathrm{Fm}$ from the interactions of $245-\mathrm{MeV}\left(1.16 \mathrm{X}\right.$ Coulomb barrier) ${ }^{40} \mathrm{Ar}$ ions with ${ }^{248} \mathrm{Cm}$. These calculations are based upon Gregorich's model of heavy ion damped reactions. The experimentally determined isotopic distributions are represented by the symbo!s connected by dashed lines.

Figure IV.9. Calculated isotopic distributions (symbols connected by solid lines) for 
$\mathrm{Bk}, \mathrm{Cf}, \mathrm{Es}$, and $\mathrm{Fm}$ from the interactions of $275 \mathrm{MeV}\left(1.16 \mathrm{X}\right.$ Coulomb barrier) ${ }^{44} \mathrm{Ca}$ ions with ${ }^{248} \mathrm{Cm}$. These calculations are based upon Gregorich's model of heavy ion damped reactions. The experimentally determined isotopic distributions are represented by the symbols connected by dashed lines.

Figure IV.10. Calculated isotopic distributions (symbols connected by solid lines) for $\mathrm{Bk}, \mathrm{Cf}, \mathrm{Es}$, and $\mathrm{Fm}$ from the interactions of $236 \mathrm{MeV}\left(1.0 \mathrm{X}\right.$ Coulomb barrier) ${ }^{44} \mathrm{Ca}$ ions with $248 \mathrm{Cm}$. These calculations are based upon Gregorich's model of heavy ion damped reactions. The experimentally determined isotopic distributions are represented by the symbols connected by dashed lines.

Figure IV.11. Calculated isotopic distributions (symbols connected by solid lines) for Es, Fm, and $\mathrm{Md}$ from the interactions of $71-\mathrm{MeV}(0.98 \times$ Coulomb barrier $){ }^{12} \mathrm{C}$ ions with ${ }^{248} \mathrm{Cm}$. The computer code ALICE was used for the calculations, which assumes compound nucleus formation followed by particle evaporation. The experimentally determined isotopic distributions are represented by the symbols connected by dashed lines.

Figure IV.12. Calculated isotopic distributions (symbols connected by solid lines) for $\mathrm{Cf}, \mathrm{Es}$, and Fm from the interactions of $84-\mathrm{MeV}$ (1.16 X Coulomb barrier) ${ }^{12} \mathrm{C}$ ions with ${ }^{248} \mathrm{Cm}$. The computer code ALICE was used for the calculations, which assumes compound nucleus formation foilowed by particle evaporation. The experimentally determined isotopic distributions are represented by the symbols connected by dashed lines.

Figure IV.13. Calculated and experimental isotopic distributions for Bk and Cf produced from the interactions of $84-\mathrm{MeV}\left(1.16 \mathrm{X}\right.$ Coulomb barrier) ${ }^{12} \mathrm{C}$ ions with ${ }^{248} \mathrm{Cn}$. Wilczynski's Sum-Rule Model was used to determine the theoretical cross sections. The experimental data are connected with dashed lines, while the calculated points are connected with solid lines.

Figure IV.14. Calculated and experimental isotopic distributions for Es and Fm 
produced from the interactions of $84 \mathrm{MeV}$ (1.16 X Coulomb barrier) ${ }^{12} \mathrm{C}$ ions with ${ }^{248} \mathrm{Cm}$. Wilczynski's Sum-Rule Model was used to determine the theoretical cross sections. The experimental data are connected with dashed lines, while the calculated points are connected with solid lines.

Figure IV.15. Calculated and experimental isotopic distributions for $\mathrm{Bk}$ and $\mathrm{Cf}$ produced from the interactions of $245-\mathrm{MeV}(1.16 \mathrm{X}$ Coulomb barrier) $40 \mathrm{Ar}$ ions with ${ }^{248} \mathrm{Cm}$. Wilczynski's Sum-Rule Model was used to determine the theoretical cross sections. The experimental data are connected with dashed lines, while the calculated points are connected with solid lines.

Figure IV.16. Calculated and experimental isotopic distributions for Es and Fm produced from the interactions of $245-\mathrm{MeV}$ (1.16 X Coulomb barrier) ${ }^{40} \mathrm{Ar}$ ions with ${ }^{248} \mathrm{Cm}$. Wilczynski's Sum-Rule Model was used to determine the theoretical cross sections. The experimental data are connected with dashed lines, while the calculated points are connected with solid lines.

Figure IV.17. Calculated and experimental isotopic distributions for $\mathrm{Bk}$ and $\mathrm{Cf}$ produced from the interactions of $275-\mathrm{MeV}\left(1.16 \times\right.$ Coulomb barrier) ${ }^{44} \mathrm{Ca}$ ions with ${ }^{248} \mathrm{Cm}$. The model developed by Hoffman and Hoffman was used to calculate the theoretical cross sections. The calculated data points based upon the geometric cross section are connected by dotted lines, the calculated cross sections multiplied by $\exp \left(0.4 E^{*}\right)$ are connected by solid lines, and the experimental cross sections are connected by dashed lines.

Figure IV.18. Calculated and experimental isotopic distributions for Es and Fm produced from the interactions of $275-\mathrm{MeV}\left(1.16 \mathrm{X}\right.$ Coulomb barrier) ${ }^{44} \mathrm{Ca}$ ions with ${ }^{248} \mathrm{Cm}$. The model developed by Hoffman and Hoffman was used to determine the theoretical cross sections. The calculated data points based upon the geometric cross section are connected by dotted lines, the calculated cross sections multiplied by $\exp \left(0.4 E^{*}\right)$ are connected by solid lines, and the experimental cross sections are 
connected by dashed lines.

Figure IV.19. Calculated and experimental isotopic distributions for Bk, Cf, Es, and Fm produced from the interactions of $245-\mathrm{MeV}\left(1.16 \mathrm{X}\right.$ Coulomb barrier) ${ }^{40} \mathrm{Ar}$ ions with ${ }^{248} \mathrm{Cm}$. The model developed by Hoffman and Hoffman was used to determine the theoretical cross sections. The calculated cross sections multiplied by $\exp \left(0.4 E^{*}\right)$ are connected by solid lines and the experimental cross sections are connected by dashed lines.

Figure IV.20. Calculated and experimental isotopic distributions for Bk, Cf, Es, and Fm produced from the interactions of $207-\mathrm{MeV}$ (1.17 X Coulomb barrier) ${ }^{31} \mathrm{P}$ ions with ${ }^{248} \mathrm{Cm}$. The model developed by Hoffman and Hoffman was used to determine the theoretical cross sections. The calculated cross sections multiplied by $\exp \left(0.4 E^{*}\right)$ are connected by solid lines and the experimental cross sections are connected by dashed lines.

Figure IV.21. Calculated and experimental isotopic distributions for Bk, Cf, Es, Fm, and $\mathrm{Md}$ produced from the interactions of $71-\mathrm{MeV}\left(1.0 \mathrm{X}\right.$ Coulomb barrier) ${ }^{12} \mathrm{C}$ ions with ${ }^{248} \mathrm{Cm}$. The model developed by Hoffman and Hoffman was used to determine the theoretical cross sections. The calculated cross sections multiplied by $\exp \left(0.4 E^{*}\right)$ are connected by solid lines and the experimental cross sections are connected by dashed lines. 


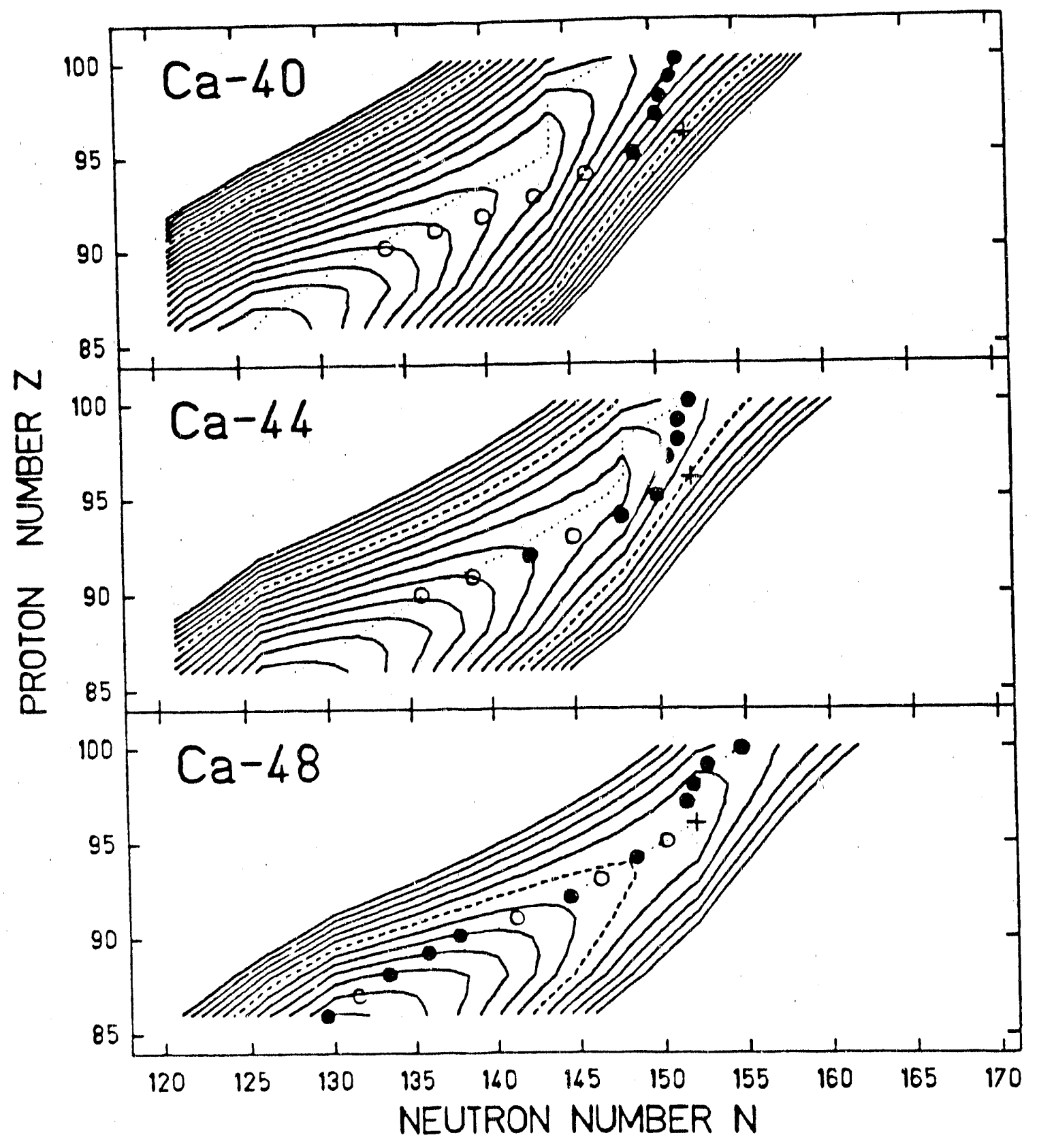

Fig. IV.1 


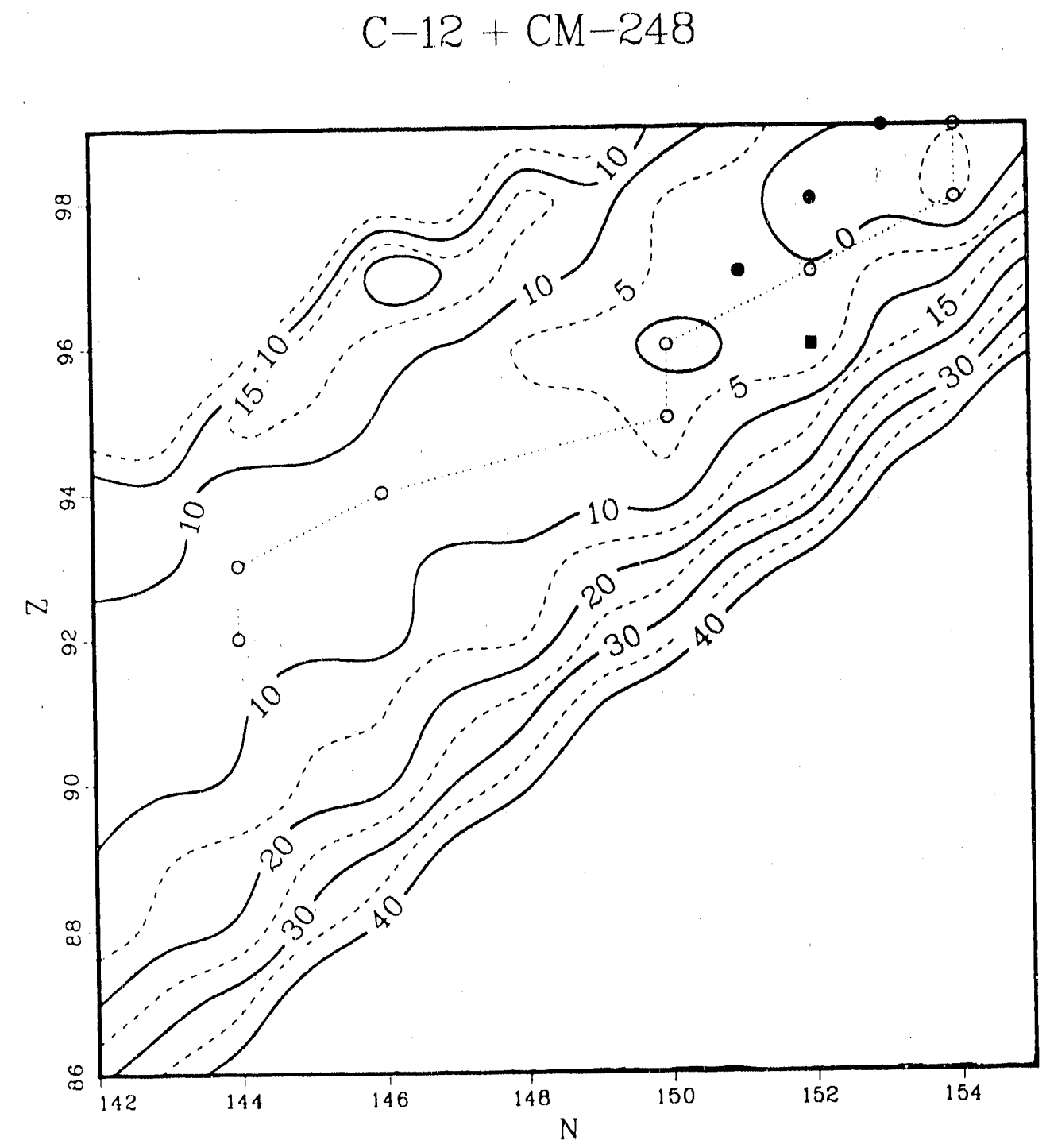

Fig. IV.2 


$$
\text { P-31+CM-248 }
$$

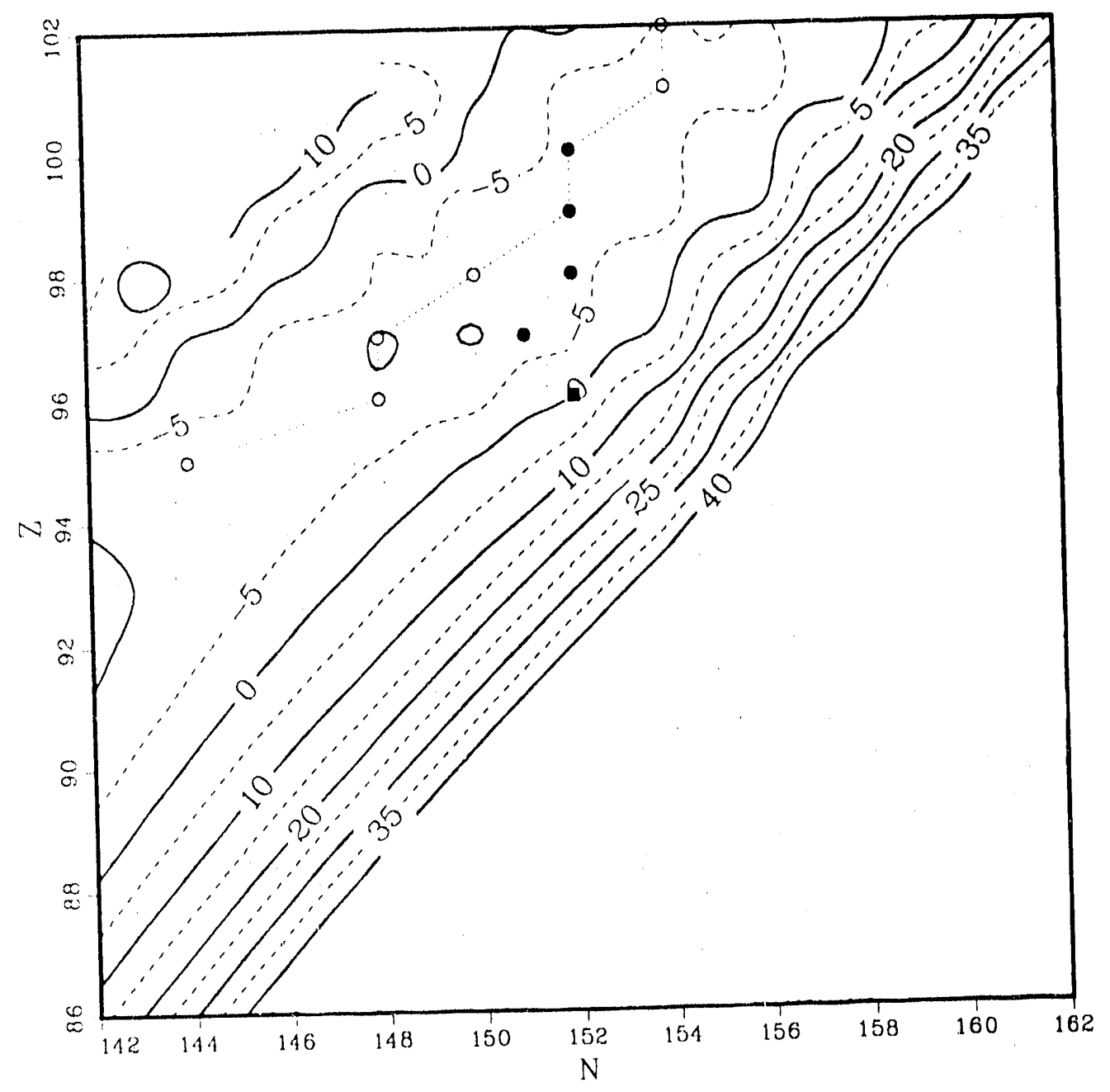

Fig. IV. 3 


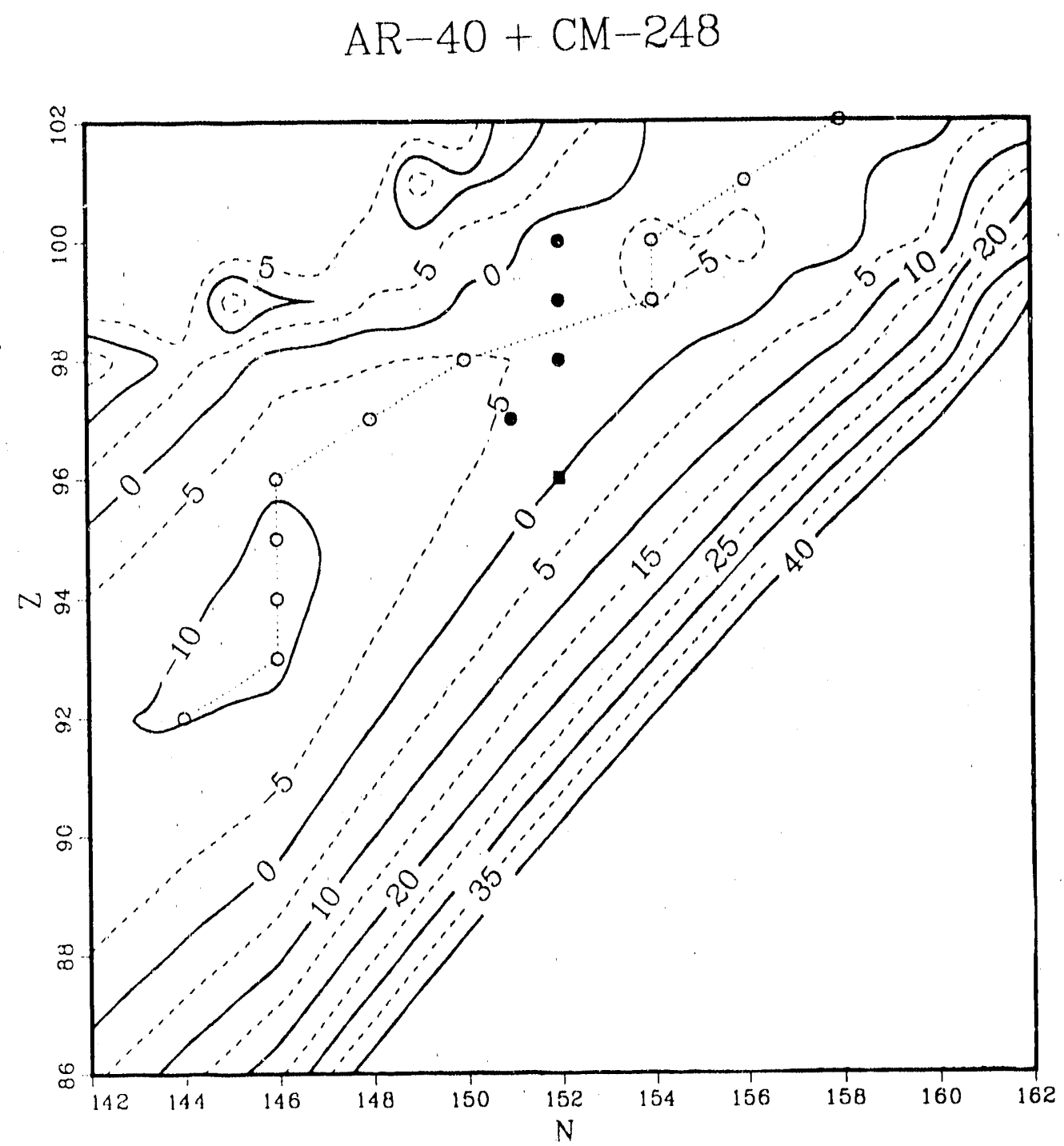

Fig. IV.4 


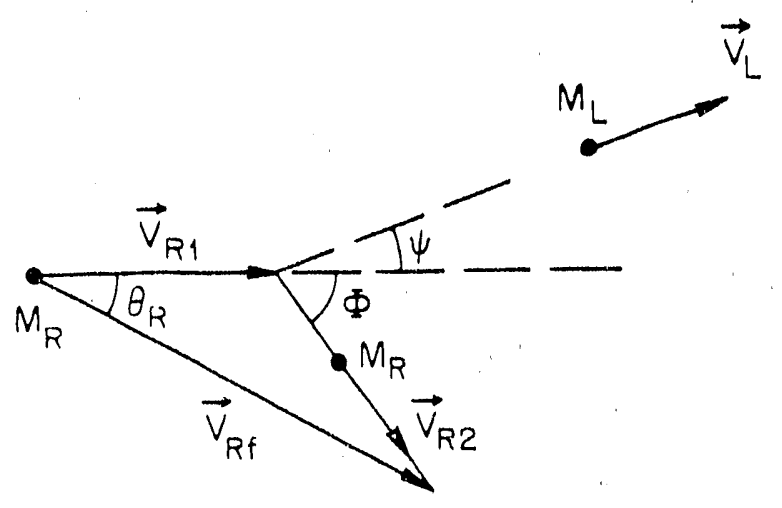

TWO-STEP TRANSFER MODEL

Fig. IV.5 


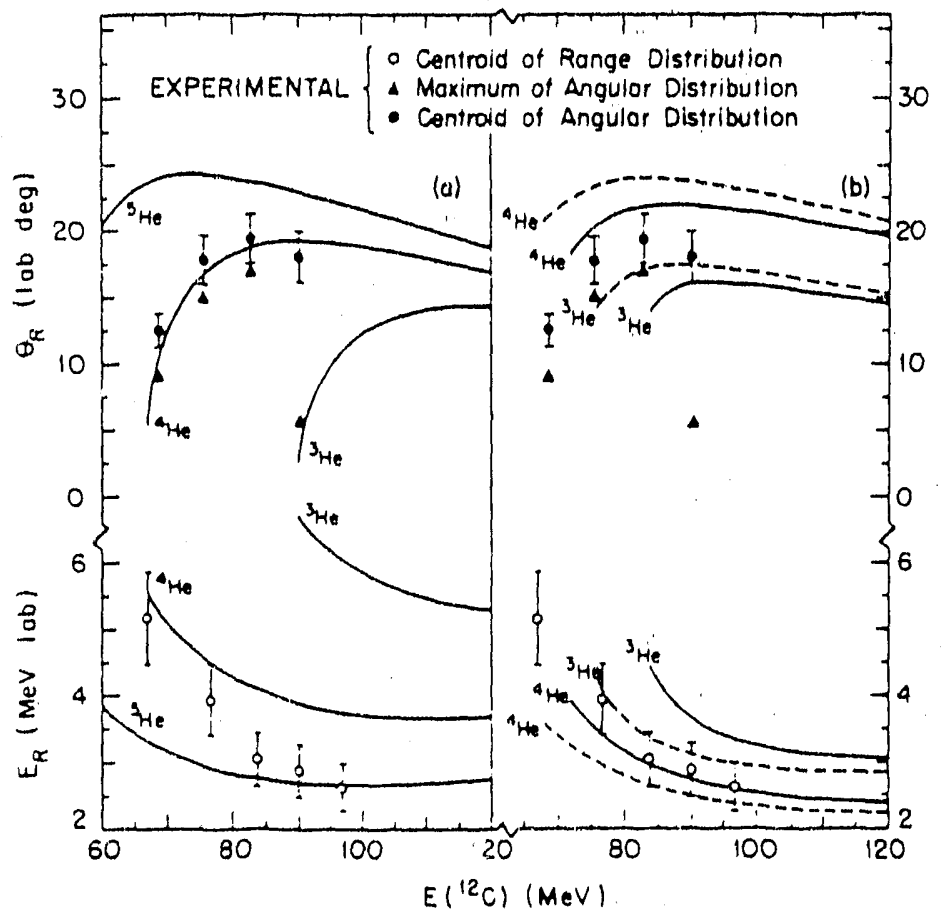

Fig. IV.6 


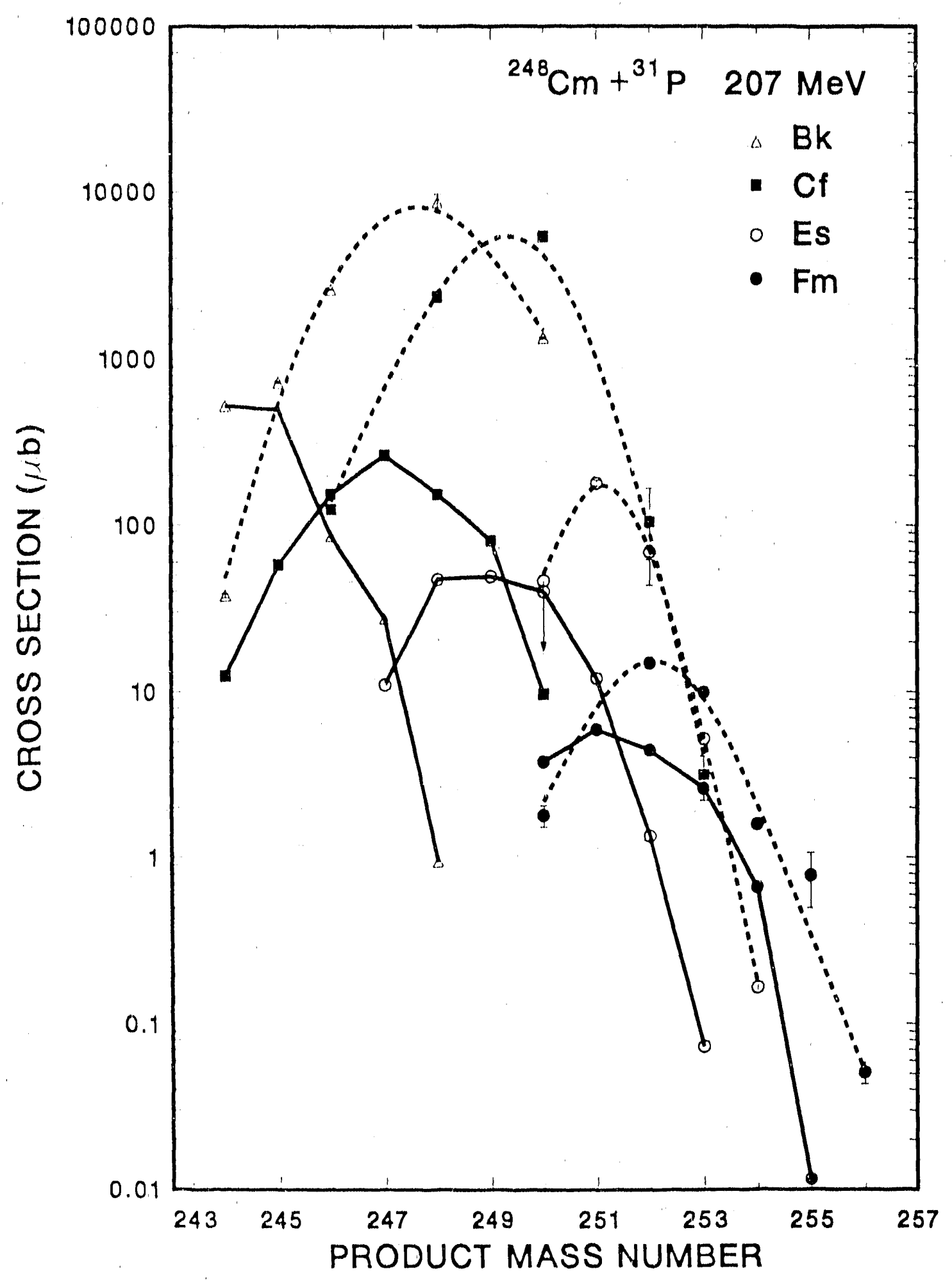

Fig. IV.7 


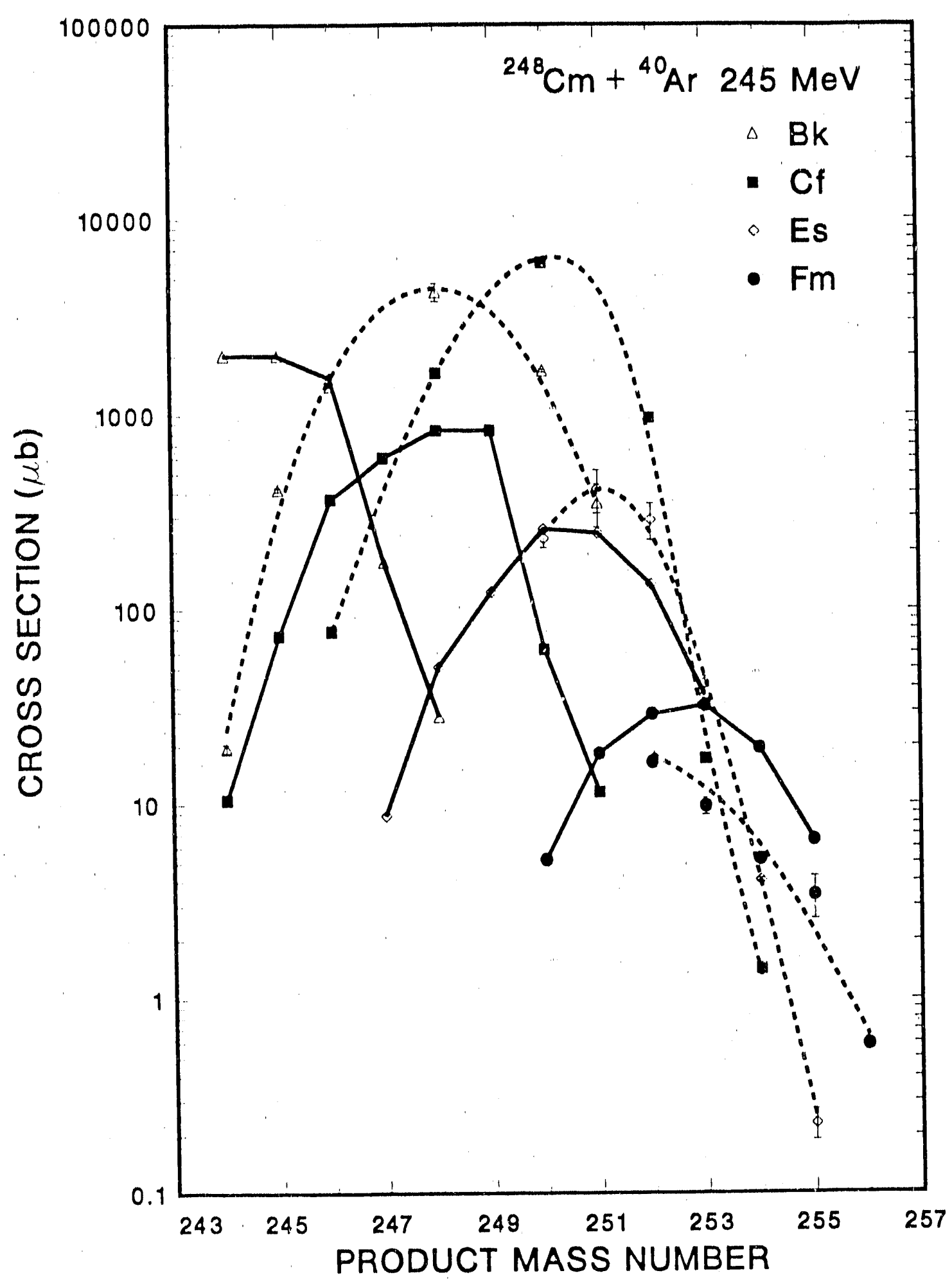

Fig. IV.8 


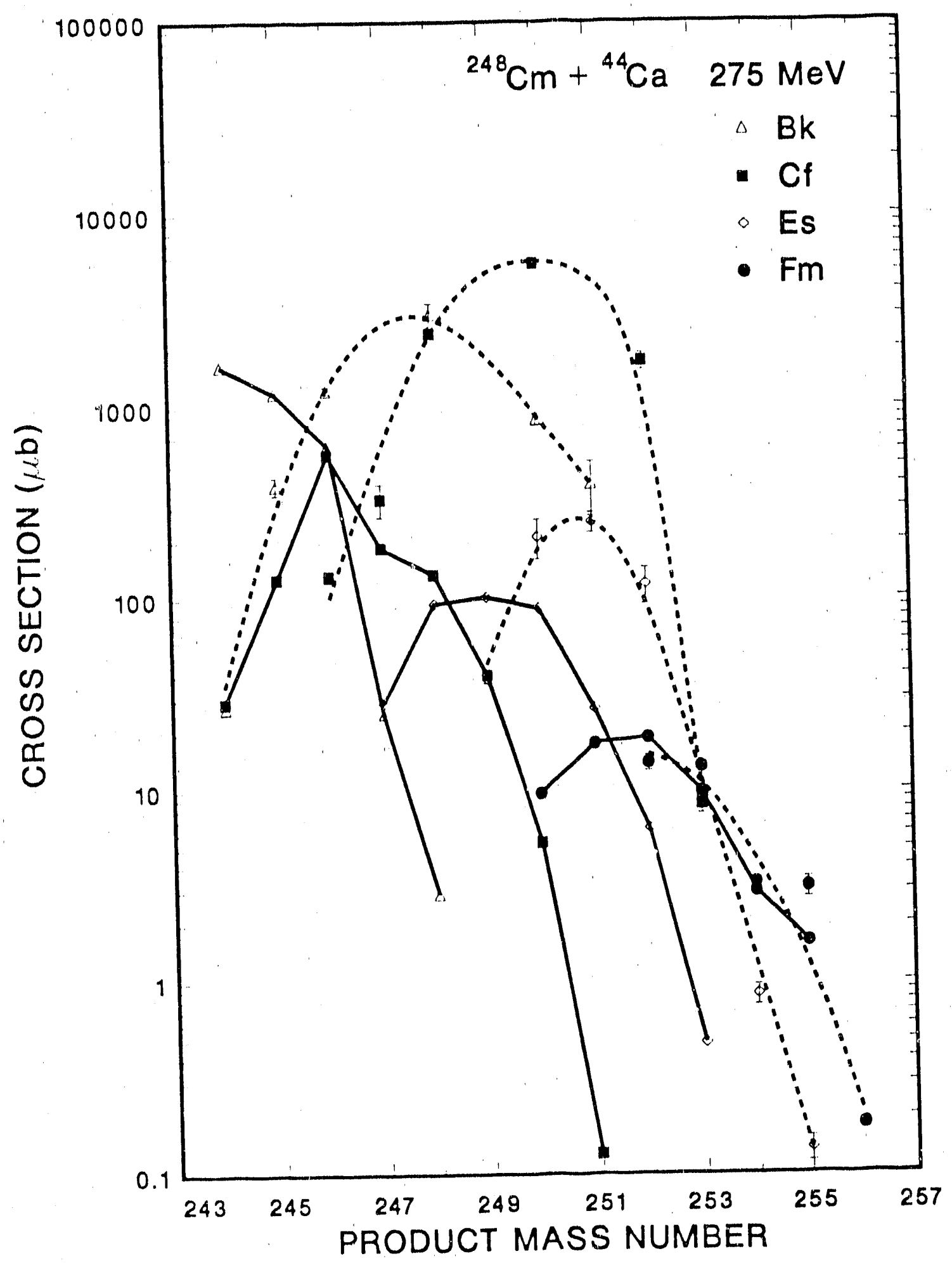

Fig. IV.9 


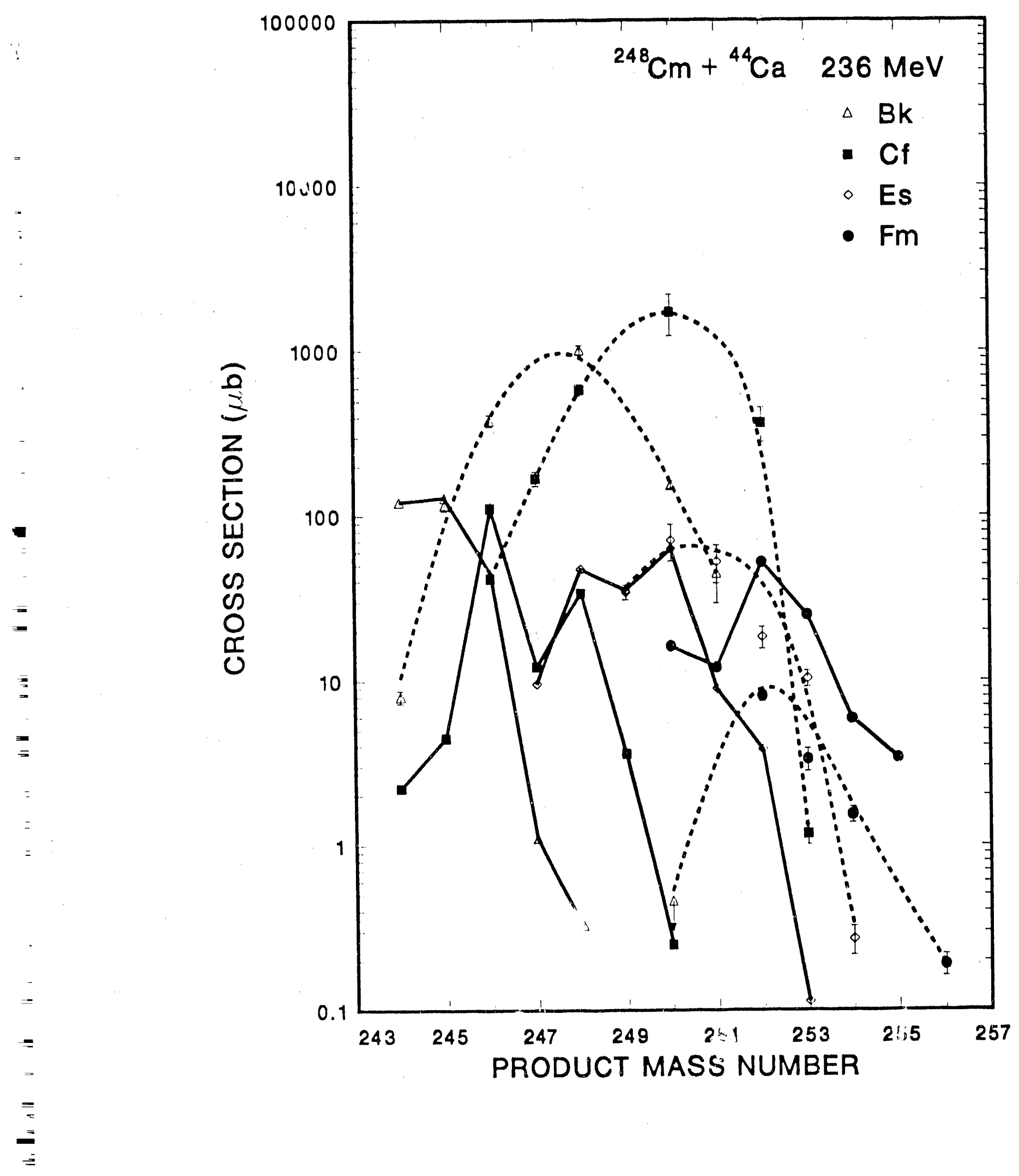

Fig. IV.10 


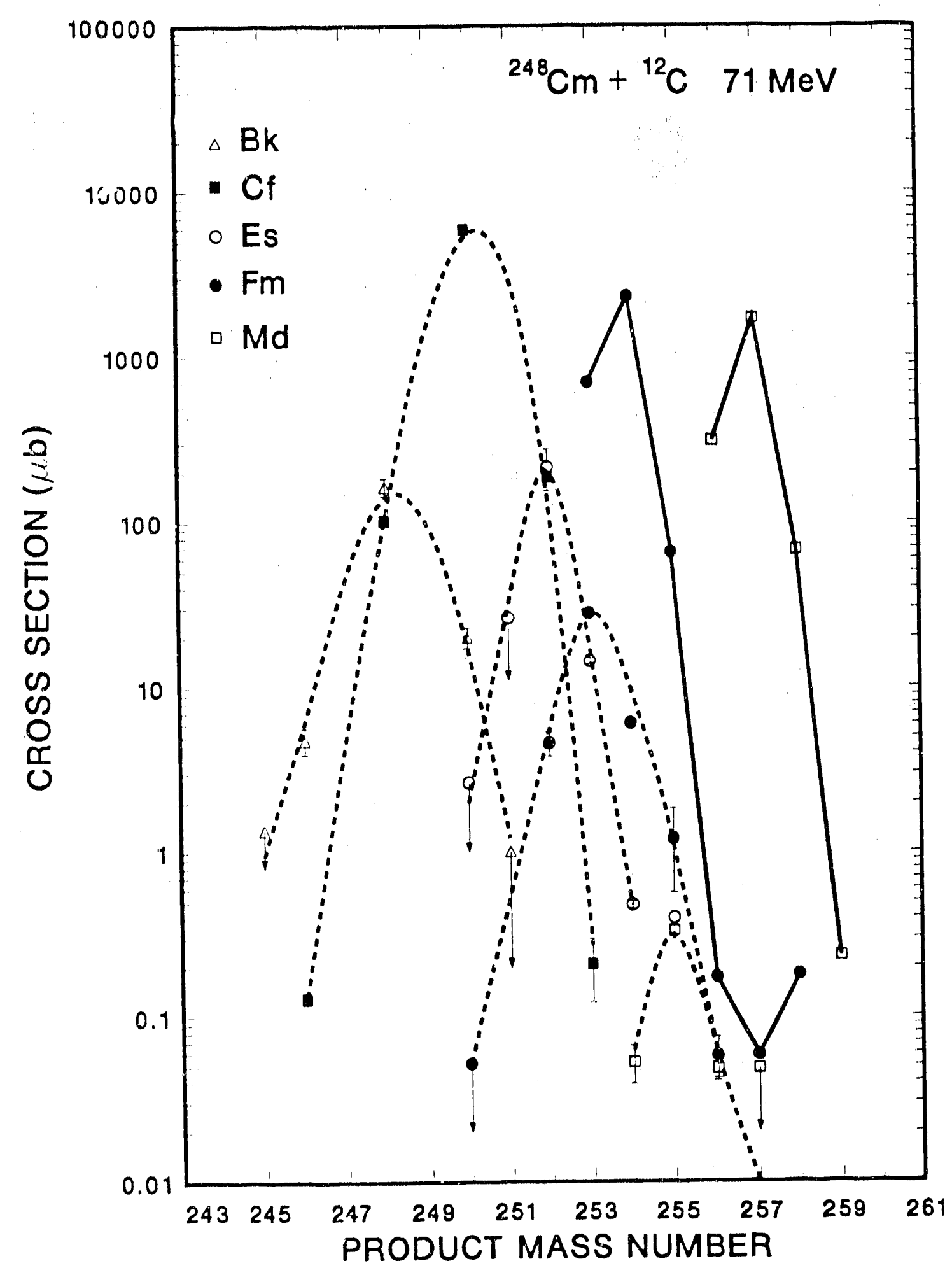

Fig. IV.11 


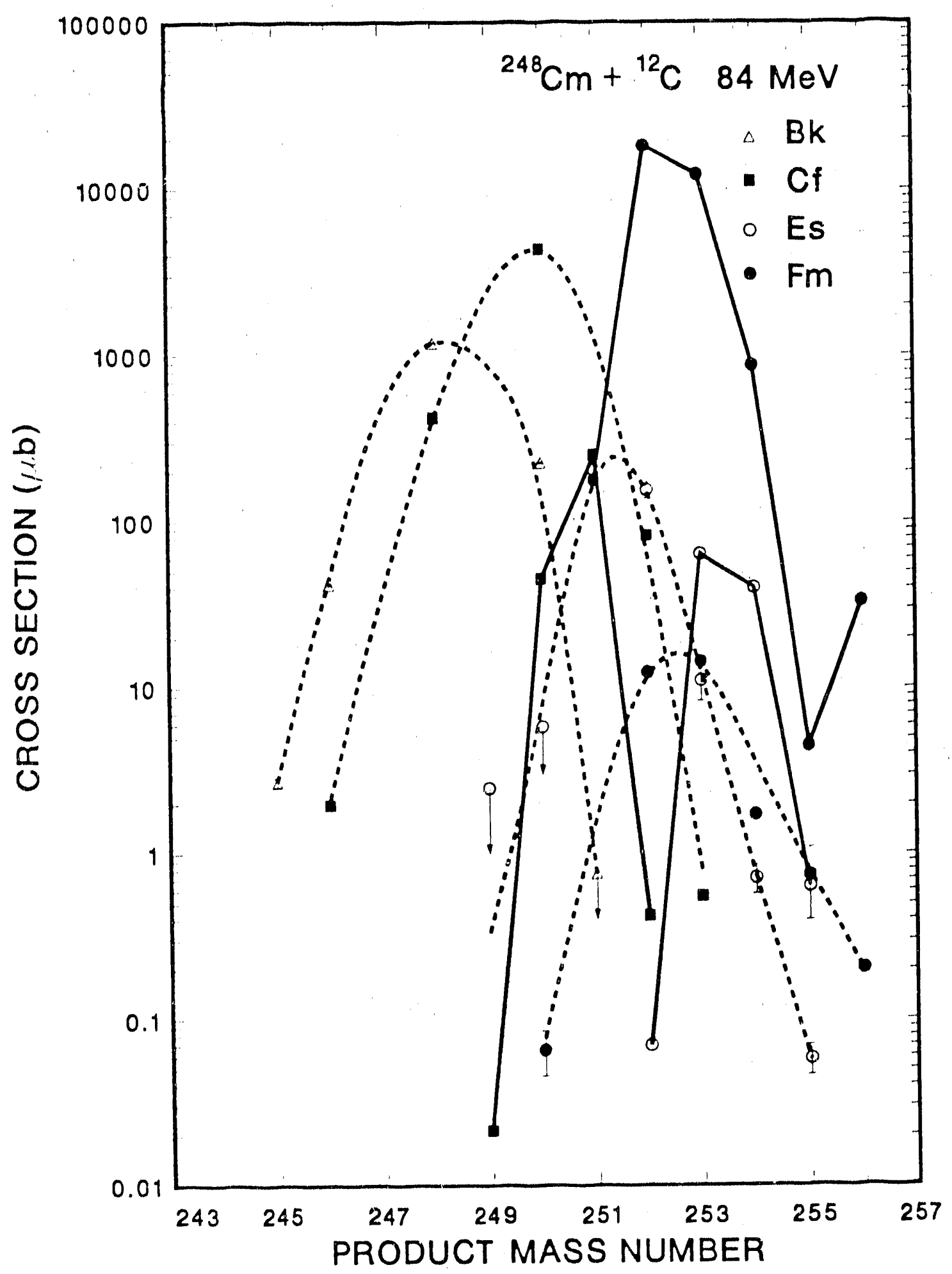

Fig. IV.12 


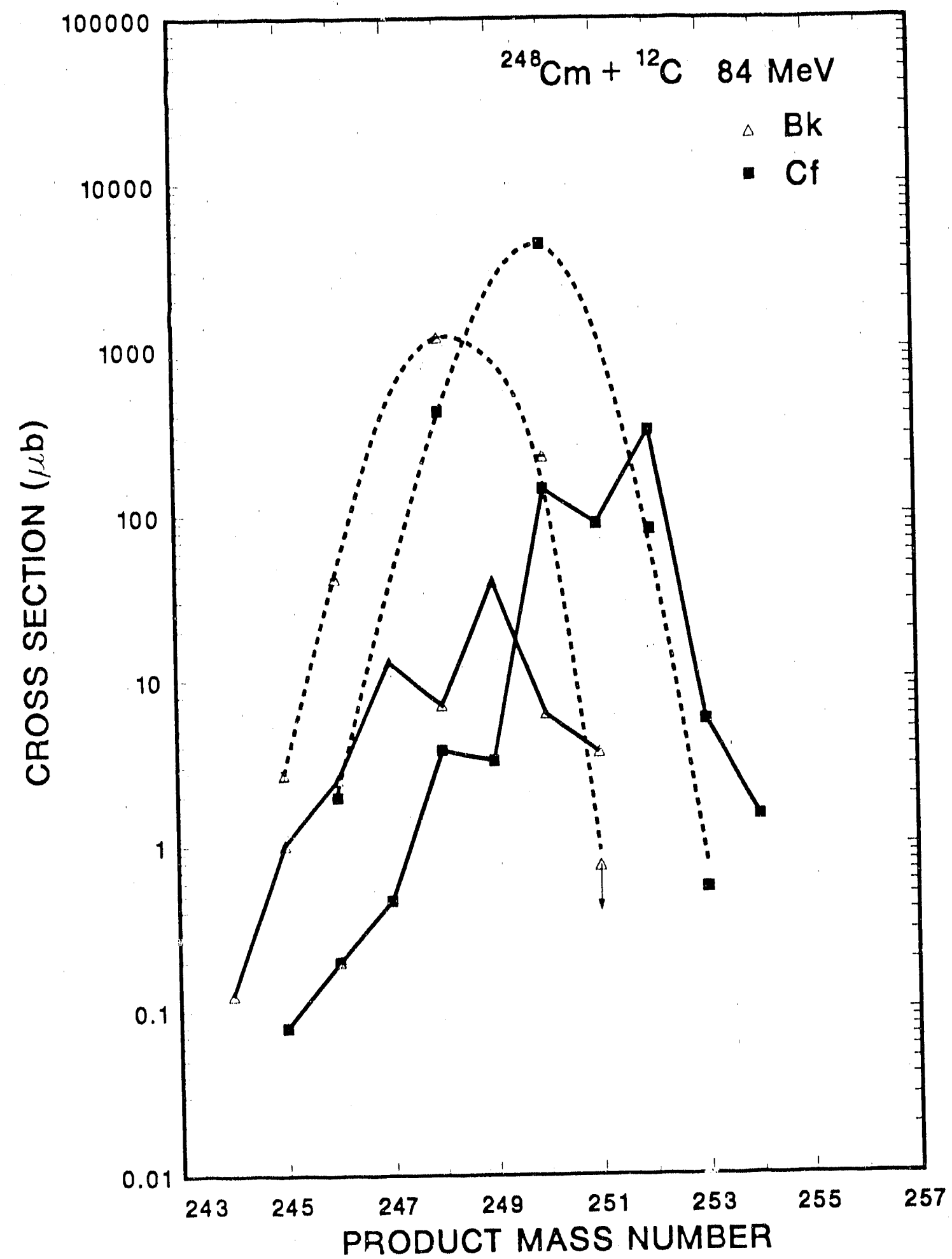

Fig. IV.13 


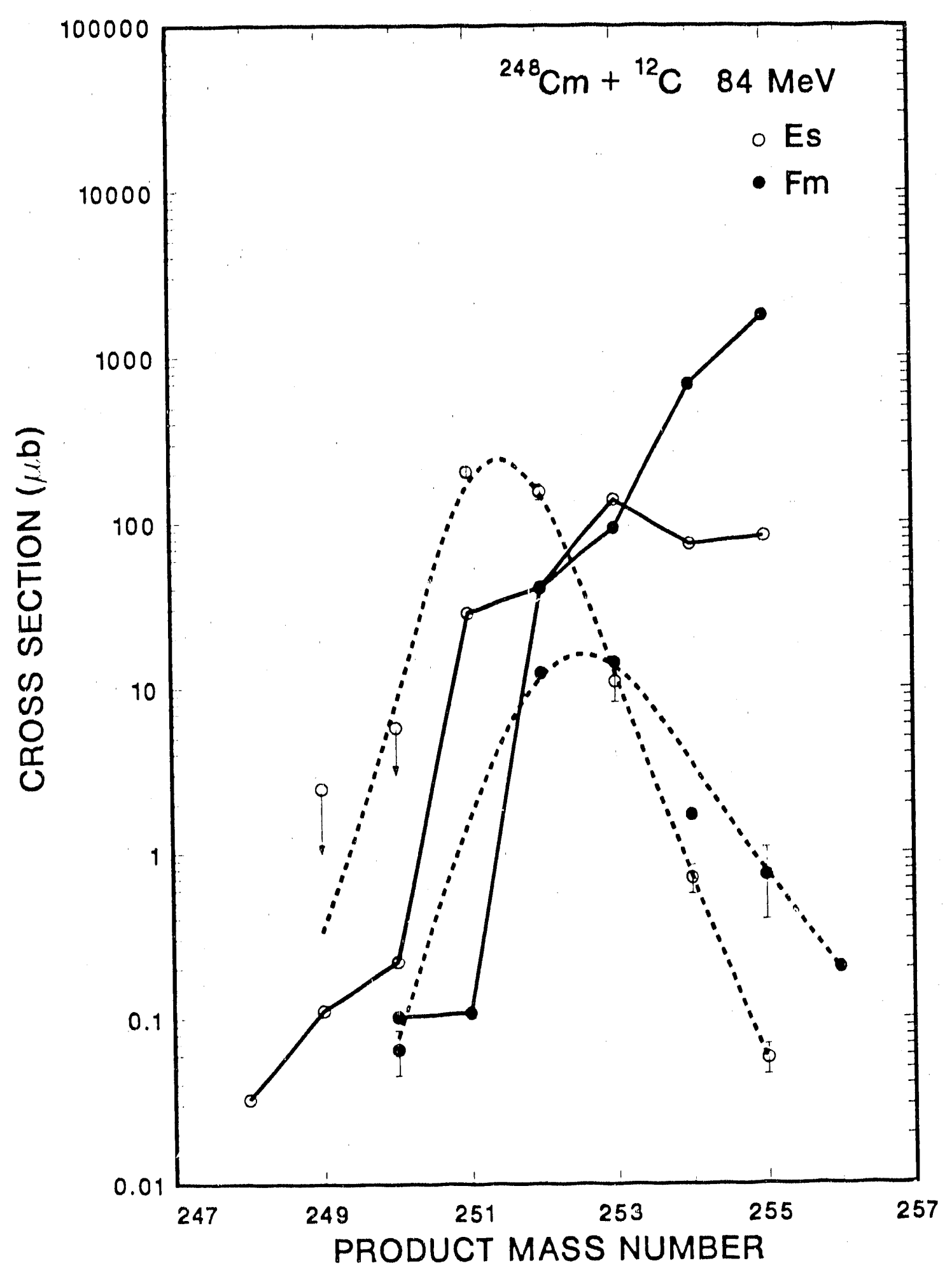

Fig. IV.14 


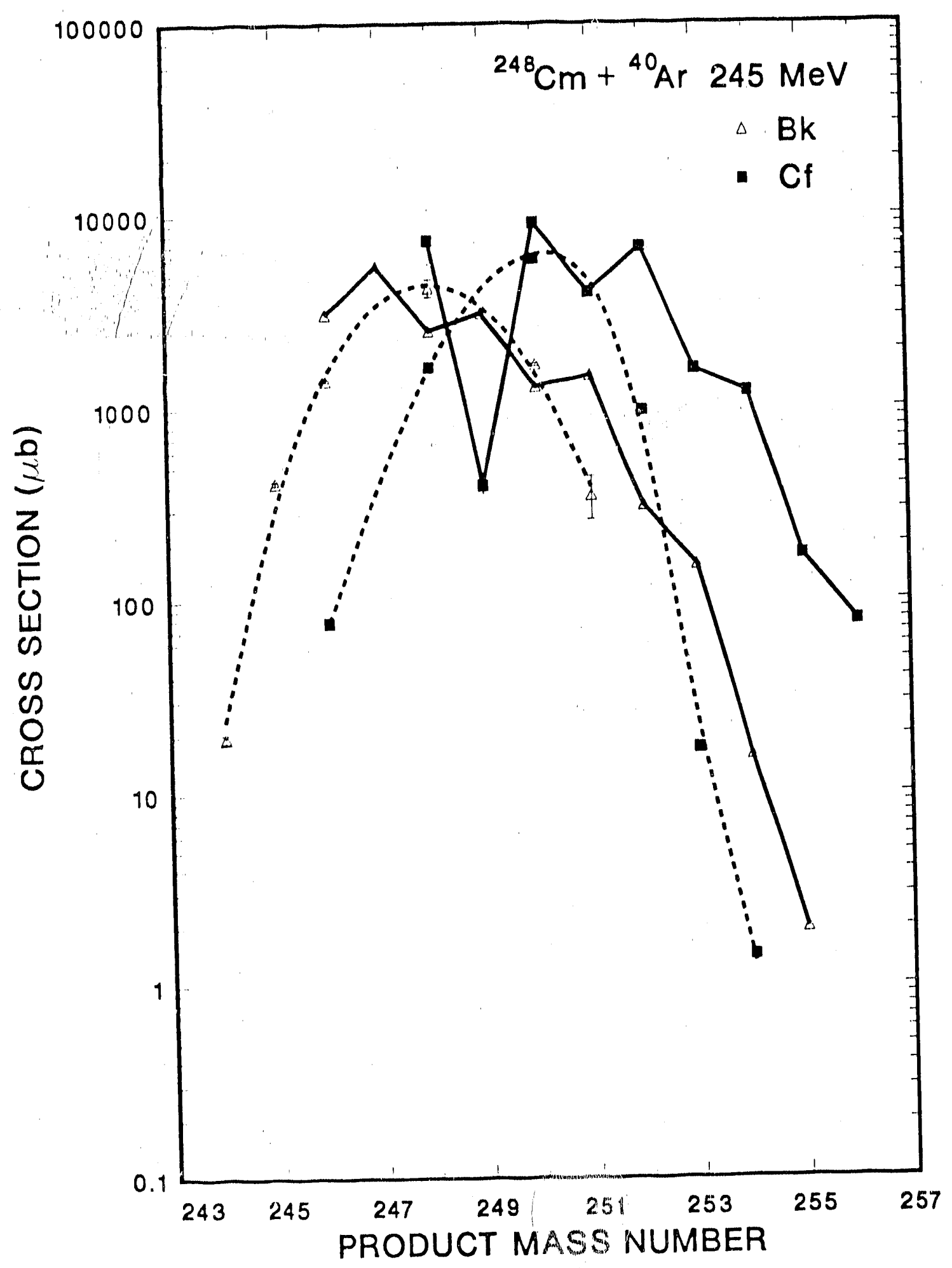

Fig. IV.15 


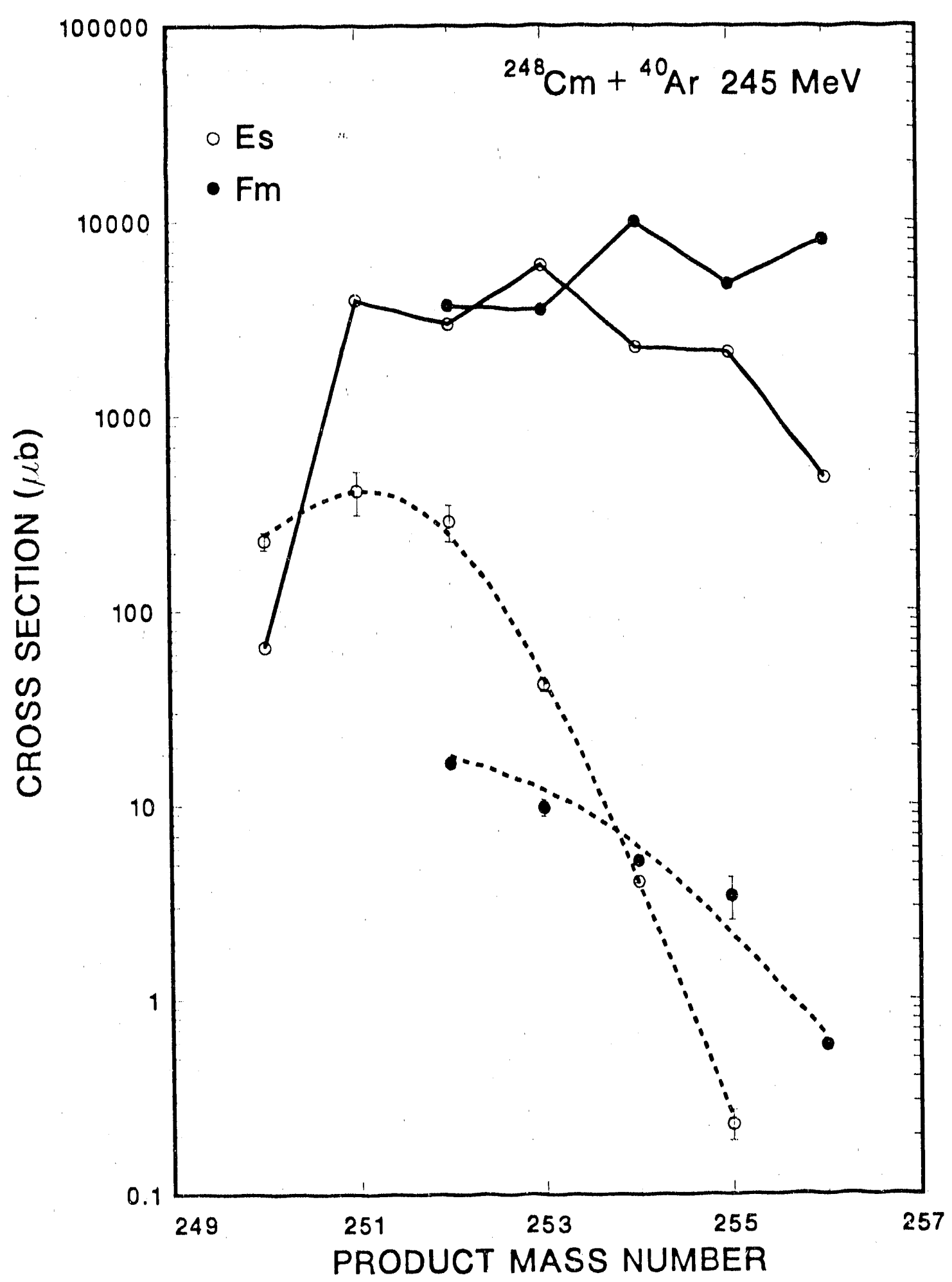

Fig. IV.16 


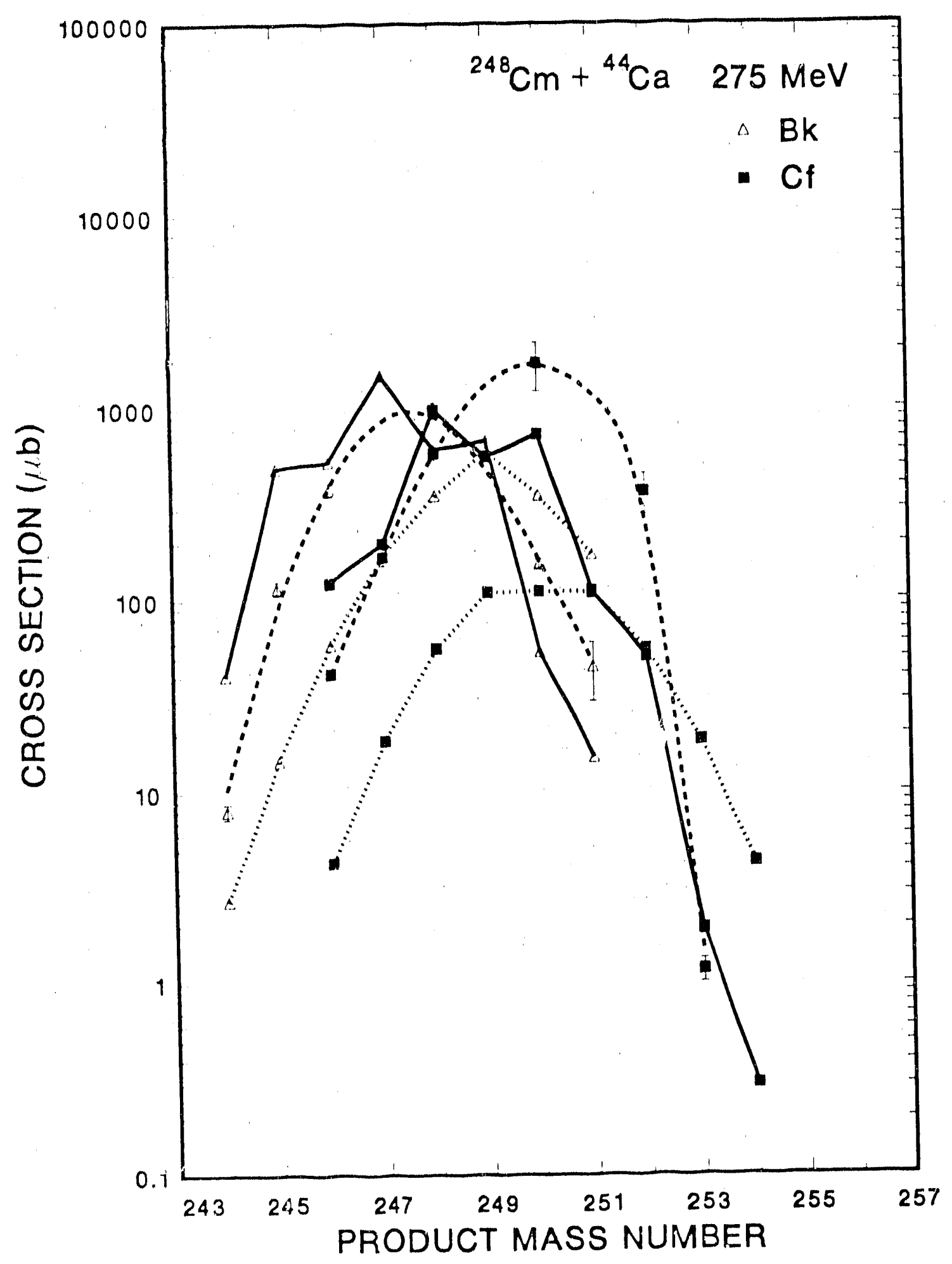

Fig. IV.17 


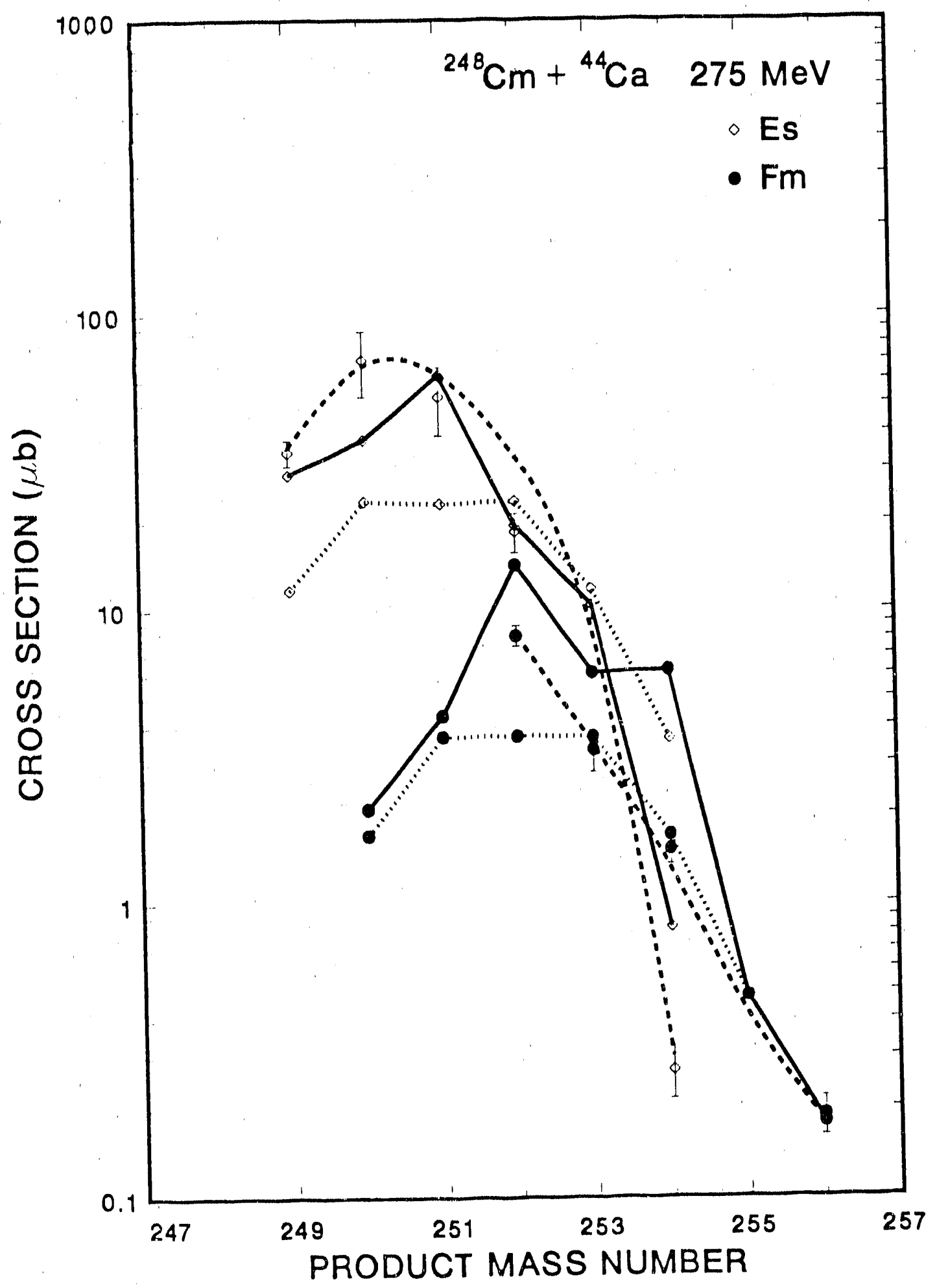

Fig. IV.18 


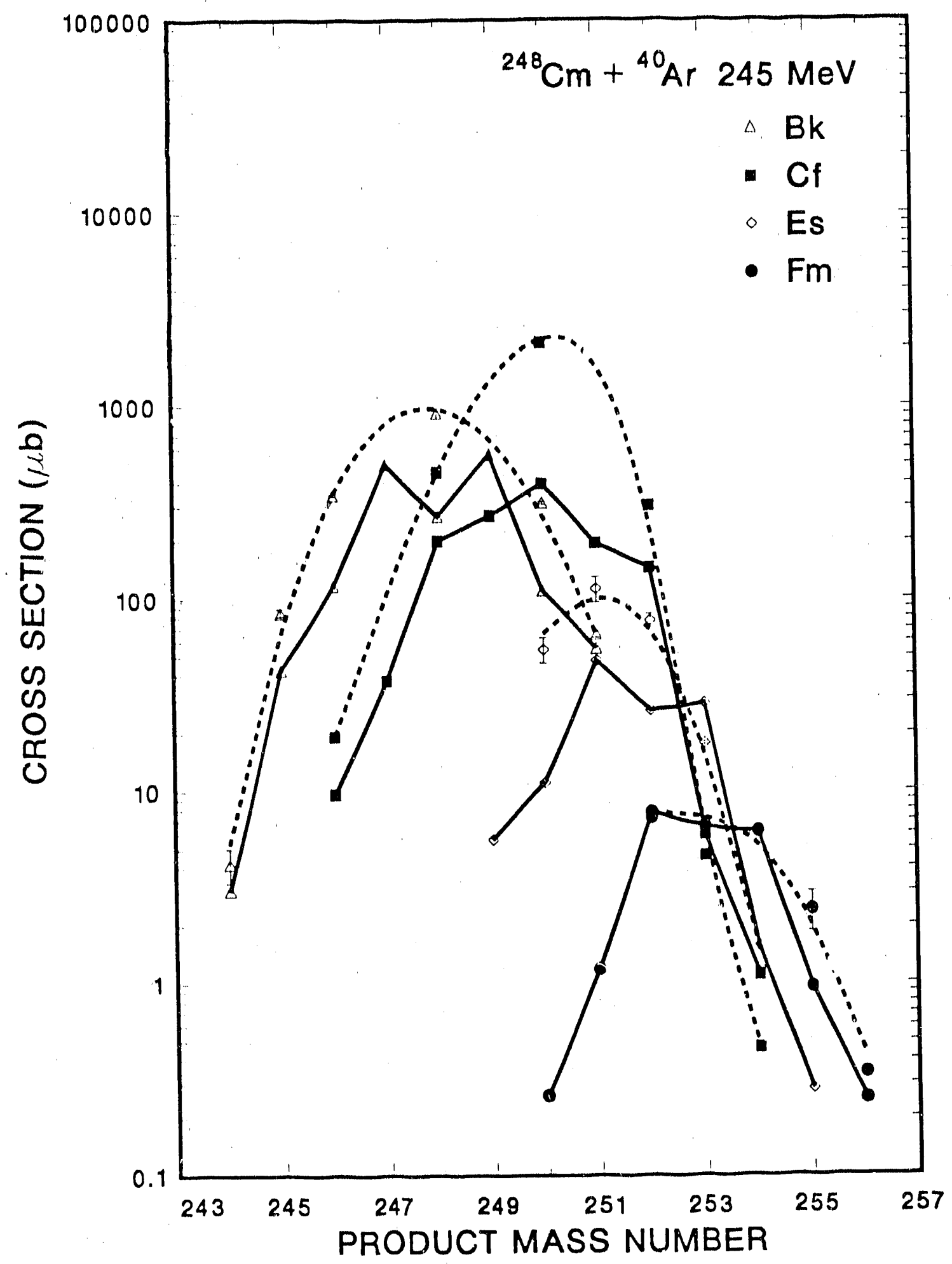

Fig. IV.19 


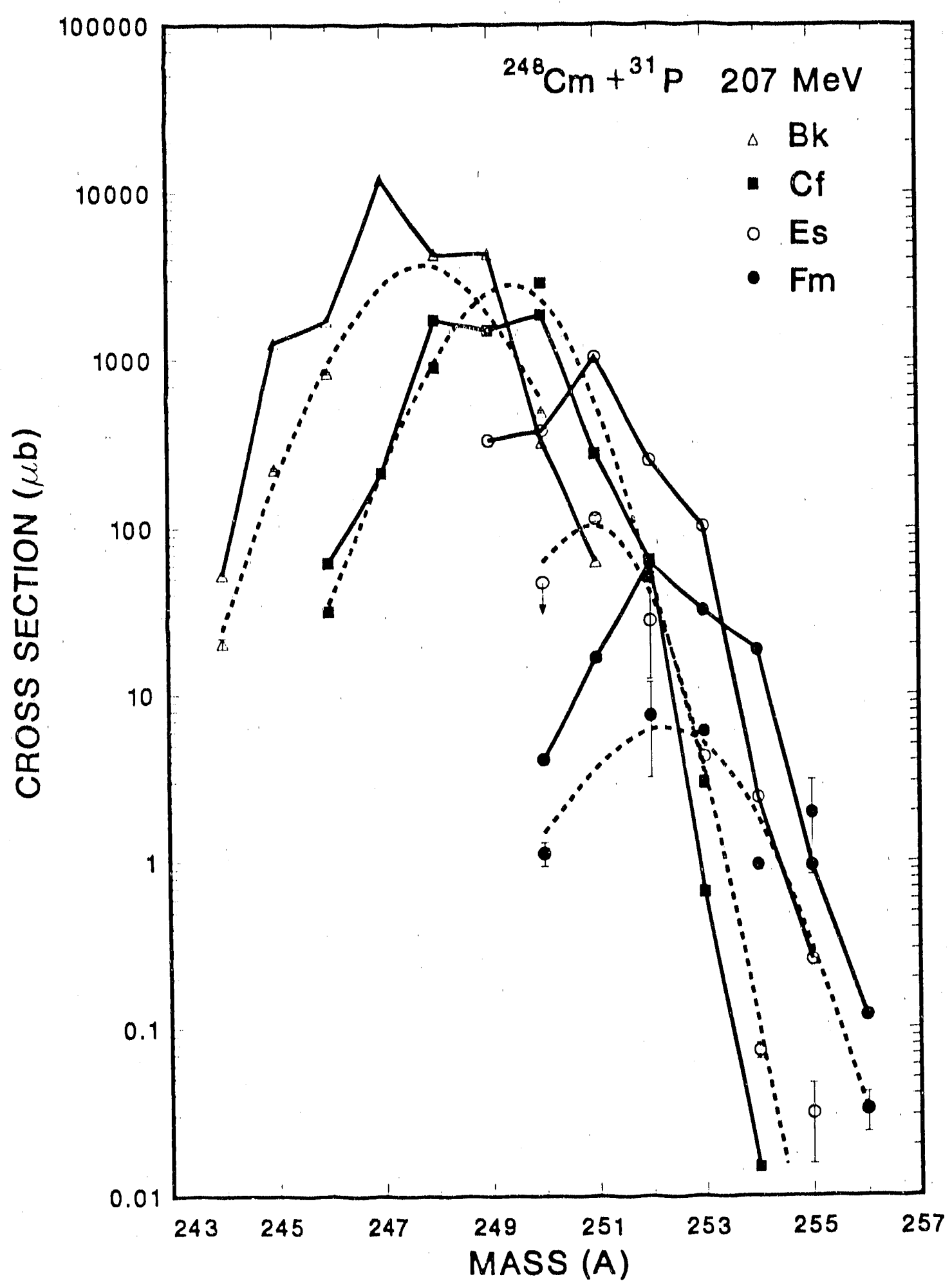

Fig. IV.20 


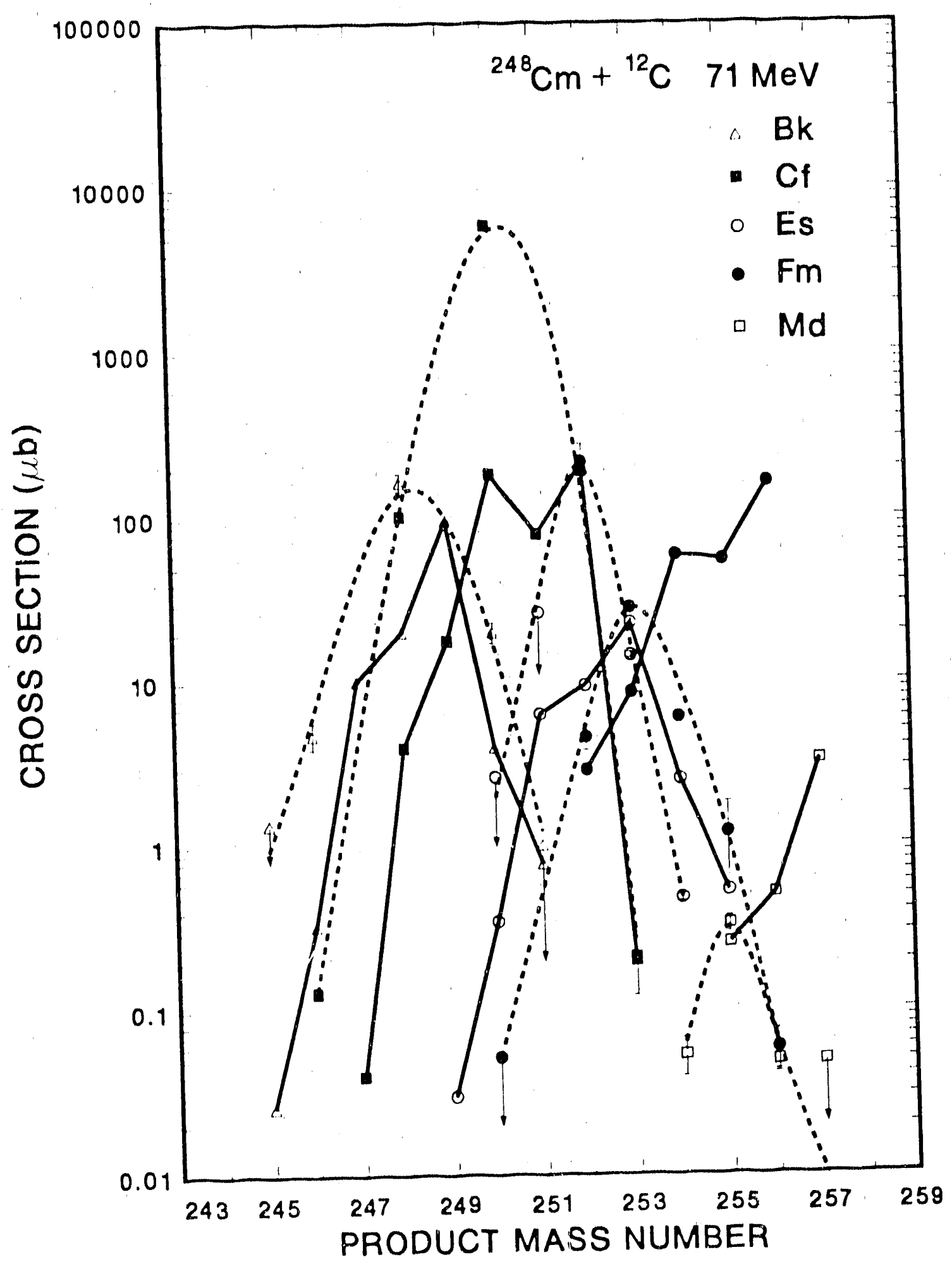

Fig. IV.21 


\section{CONCLUSIONS}

${ }^{248} \mathrm{Cm}$ was bombarded with ${ }^{12} \mathrm{C},{ }^{31} \mathrm{P},{ }^{40} \mathrm{Ar}$, and ${ }^{44} \mathrm{Ca}$ ions at energies of $0.98,1.06$, $1.16,1.25$, and $1.35 \times$ Coulomb barrier. The recoiling reaction products were collected by copper or gold catcher foils. A radiochemical procedure was used to obtain separate fractions of $\mathrm{Bk}, \mathrm{Cf}, \mathrm{Es}, \mathrm{Fm}$, and $\mathrm{Md}$. A $\mathrm{He} / \mathrm{KCl}$ jet was used to transport the recoiling No nuclides produced in the ${ }^{12} \mathrm{C}$ system to a rotating wheel system.

The isotopic distributions of the above target reaction products $\left(Z_{\text {product }}>Z_{\text {target }}\right)$ were found to be essentially symmetric about the maximum with full-widths-at-halfmaximum (FWHM) of $<1.5$ to 3.3 mass units. These results are similar to FWHM values found previously for other heavy ion $-248 \mathrm{Cm}$ systems. The peaks of the isotopic distributions generally occur for those reaction channels involving the apparent transfer of the fewest number of nucleons for which the calculated excitation energy is a positive quantity. The maxima of the excitation functions occur at those projectile energies which are consistent with the calculated reaction barriers based upon a binary reaction mechanism.

Potential Energy Surfaces (PES) do a relatively poor job of calculating the primary mass distributions of the above target actinide reaction products for the systems studied. A model based upon heavy ion damped reactions was used to calculate the final isotopic distributions of the above target actinide products. This model does an inadequate job of reproducing the $\mathrm{Bk}, \mathrm{Cf}$, and Es experimental isotopic distributions of the ${ }^{31} \mathrm{P},{ }^{40} \mathrm{Ar}$, and ${ }^{44} \mathrm{Ca}$ systems. The model, however does a good job reproducing the Fm experimental isotopic distributions. In most cases, the experimental peak centroids of the isotopic distributions lie or the neutron-rich side of the calculated peak centroids, indicating that $N / Z$ equilibration is not being achieved in these reactions. The damped reaction model consistently gives cross sections which are too low for many of the above target actinide reaction products, especially for isotopes of $\mathrm{Bk}, \mathrm{Cf}$, and Es. Since the cross sections for 
isotopes of $\mathrm{Bk}, \mathrm{Cf}$, and $\mathrm{Es}$ from the ${ }^{12} \mathrm{C},{ }^{31} \mathrm{P},{ }^{40} \mathrm{Ar}$, and ${ }^{44} \mathrm{Ca}$ systems do not appreciably decrease over the energy range investigated, one can conclude that these products are being formed with little or no excitation energy. A quasi-elastic mechanism is thus probably responsible for the production of isotopes of $\mathrm{Bk}, \mathrm{Cf}, \mathrm{Es}$, and perhaps some Fm.

The computer code ALICE, based upon compound nucleus formation and subsequent particle evaporation, was used to calculate actinide production cross sections for the ${ }^{12} \mathrm{C}$ system. ALICE does a very poor job of reproducing the isotopic distributions of the above target products, thus indicating that a compound nucleus mechanism is not involved in the production of these nuclides.

Hoffman and Hoffman developed a model utilizing a classical geometric cross section calculation for binary reactions. The calculated isotopic distributions based upon this model agree quite well with the experimental isotopic distributions. This model, however, does show some discrepancies with the experimental isotopic distributions from the ${ }^{12} \mathrm{C}$ system.

'Transfer reactions provide a useful tool for the production of "cold", neutron-rich actinides. It appears that the ${ }^{40} \mathrm{Ca}-{ }^{248} \mathrm{Cm}$ system might be promising for the production of 274111 . The $E_{x}$ value for this reaction channel is $0.5 \mathrm{MeV}$. Another possibility is the production of $291114\left(E_{X}=0.8 \mathrm{MeV}\right)$ employing the ${ }^{48} \mathrm{Ca}-248 \mathrm{Cm}$ system. Perhaps the ${ }^{40} \mathrm{Ca}-{ }^{249} \mathrm{Bk}$ system could be used to produce 272111 or 278111 which have $E_{X}$ values of 0.9 and $1.4 \mathrm{MeV}$ respectively. With the choice of such heavy projectiles at energies near the Coulomb barrier, the fraction of excitation energy transferred to the target-like fragment should be low, thus producing "cold" neutron-rich actinides. Hence, by choosing the appropriate target-projectile-energy combinations, transfer reactions can be used to produce new, neutron-rich actinides and possibly reach the "island of stability." 


\section{APPENDIX A}

\section{Catcher foil collection efficiency code}

PROGRAM CATFOIL

WRITE $\left(*{ }^{*}\right)^{\prime}$ This program calculates the probablity of

WRITE $\left(*^{*},\right)^{\prime}$ 'hitting the catcher foil for reaction'

WRITE $(*, *)^{*}$ 'products emitted at angles between one'

WRITE $(*, *)$ 'and eighty-nine degrees inclusive with'

WRITE $(*, *)^{\prime}$ 'respect to the beam axis as a function'

WRITE $(*, *)$ 'of angle.'

WRITE $(*, *)^{\prime}$ Equations taken from K. Moody Ph.D. Thesis,'

WRITE $(*, *)^{\prime}$ LBL-16249, PAGE 84.'

WRITE $(*, *)$

WRITE $(*, *)^{\prime}$ Enter the radius of the catcher foil (RFOIL),'

WRITE $(*, *)^{\prime}$ the radius of the target (RTARGET), and'

WRITE $(*, *)^{\prime}$ the distance between the target and catcher'

WRITE $(*, *)^{\prime}$ foil (D), in that order.'

WRITE $(*, *)^{\prime}$ Use $\mathrm{mm}$ as the unit of choice.'

WRITE $(*, *)$

READ $(*, *)$ RFOIL,RTARGET,D

WRITE $(*, *)$

$\mathrm{PI}=3.14159$

PROBMISS $=0.0$

PROBHIT $=0.0$

WRITE $(*, 100)$

100 FORMAT (' What do you want to call the output file?')

OPEN (2,File =' ',Status='New')

WRITE $(2,105)$

105 FORMAT (' ANGLE PROB. MISS PROB. HIT')

C Convert degrees into radians (Multiply by PI/180)

DO $1, \mathrm{~J}=1,89$

TRAD $=$ FLOAT $(J) * P I / 180$.

$\mathrm{PROBHIT}=0$.

PROBMISS $=0$.

DO 2, $K=1,1000$

TR=FLOAT $(\mathrm{K}) *$ RTARGET $/ 1000$.

C IF RECOILING PRODUCTS MISS ALL OF THE TIME

IF ((RFOIL+TR-D*TAN(TRAD)).LT.0)THEN

$P R O B M=1$.

$P R O B H=0$.

C IF RECOILING PRODUCTS HIT ALL THE TIME

ELSE IF ((RFOIL-TR-D*TAN(TRAD)).GE.0)THEN

$\mathrm{PROBM}=0$.

$\mathrm{PROBH}=1$.

C THE OTHER CASES (SOMETIMES HITS AND MISSES) 
ELSE

$\mathrm{A} 1=((\mathrm{D} * * 2) *(\mathrm{TAN}(\mathrm{TRAD}) * \mathrm{TAN}(\mathrm{TRAD})))-\left(\mathrm{RFOIL}^{* * 2}\right)+\left(\mathrm{TR}^{* *} 2\right)$

$\mathrm{B} 1=\left(2 . *{ }^{*} \mathrm{TR}^{*} \mathrm{D} * \mathrm{TAN}(\mathrm{TRAD})\right)$

$\mathrm{C} 1=\mathrm{A} 1 / \mathrm{B} 1$

$\mathrm{PROBM}=(1 . / \mathrm{PI}) *((\mathrm{ASIN}(\mathrm{C} 1)+\mathrm{PI} / 2)$.

$\mathrm{PROBH}=1 .-\mathrm{PROBM}$

ENDIF

PROBHIT $=$ PROBHIT + PROBH $*$ TR

$\mathrm{PROBMISS}=\mathrm{PROBMISS}+\mathrm{PROBM} * \mathrm{TR}$

PROBT $=$ PROBHIT + PROBMISS

2 CONTINUE

WRITE $(2,200)$ J,PROBMISS/PROBT,PROBHIT/PROBT

200 FORMAT (2X,I5,7X,F6.4,8X,F6.4)

1 CONTINUE

STOP

END 


\section{REFERENCES}

[ABU80] A.B. Abul-Magd, Z. Physik A298, 143 (1980).

[ART71] A.G. Artukh, V.V. Avdeichikov, J. Erö, G.F. Gridnev, V.L. Mikheev, V.V. Volkov, and J. Wilczynski, Nucl. Phys. A160, 511 (1971).

[BAU78] H.R. Bauer, G.D. Christian, and J.E. O'Reilly, Instrumental Analysis (Allyn and Bacon Inc. Boston 1978).

[BAY73] R.D. Baybarz, J. Inorg. Nucl. Chem., 35, 4149 (1973).

[BEE87] J. Beene, private communication, (1987).

[BIM72] R. Bimbot, D. Gardes, and M.F. Rivet, Nucl. Phys. A189, 193 (1972).

[BLA71] M. Blann, Phys. Rev. Lett., 27, 337 (1971).

[BLA72] M. Blann, Phys. Rev. Lett., 28, 757 (1972).

[BLA82] M. Blann and J. Bisplinghoff, Lawrence Livermore National Laboratory Report UCID-19614 (1982).

[BLO77] J. Blocki, J. Randrup, W.J. Swiatecki, and C.F. Tsang, Ann. Phys. 105, 427 (1977).

[BOH39] N. Bohr and J.A. Wheeler, Phys. Rev. 56, 426 (1939).

[BRA86] S.G. Bratsch and J.J. Lagowski, J. Phys. Che.iı. 90, 307 (1986).

[BRI72] D.M. Brink, Phys. Lett. 40B, 37 (1972).

[BRI79] H.C. Britt, in Proceedings of the Fourth International Atomic Energy Symposium on Physics and Chemistry of Fission, Jülich, 1979 (IAEA, Vienna, 1980), Vol. I, p. 3.

[PRI80] H.C. Britt, E. Cheifetz, D.C. Hoffman, J.B. Wilhelmy, R.J. Dupzyk, and R.W. Lougheed, Phys. Rev. C 21, 761 (1980).

[BRO78] E. Browne, J.M. Dairiki, and R.E. Doebler, Table of the Isotopes, Second Edition (J. Wiley and Sons, Inc., New York 1978).

[CHA87] R.M. Chasteler, R.A. Henderson, D. Lee, K.E. Gregorich, M.J. Nurmia, R.B. Welch, and D.C. Hoffman, Phys. Rev. C 36, 1820 (1987). 
[CHO56] G.R. Choppin, B.G. Harvey, and S.G. Thompson, J. Inorg. Nucl. Chem. 2, 66 (1956).

[CHO56A] G.R. Choppin and R.J. Silva, J. Inorg. Nucl. Chem. 3, 153 (1956).

[CHO61] G.R. Choppin abd J.A. Chopoorian, J. Inorg. Nucl. Chem. 22, 97 (1961).

[COO52] G.B. Cook, and J.F. Duncan, Modern Radiochemical Practice (Oxford at the Clarendon Press, Oxford 1952).

[CRC75] Handbook of Chemistry and Physics, 56th Edition (CRC Press, Inc., Cleveland 1975).

[CUM63] J.B. Cumming, Brookhaven National Laboratory Report BNL-6470 (1963).

[DOR89] J.G. Dorsey and K.A. Dill, Chem. Rev. 89, 331 (1989).

[DOS85] T. Dossing and J. Randrup, Nucl. Phys. A433, 215 (1985).

[DYE61] F.F. Dyer and G.W. Liddicotte, The Radiochemistry of Copper NAS-NS-3027 (1961).

[EIS85] J.M. Eisenberg and W. Greiner, Nuclear Models Collective and Single Particle Phenomenon, Second Edition ( North Holland Publishing Co., Amsterdam 1975).

[ESK70] P. Eskola, K. Eskola, M. Nurmia, and A. Ghiorso, Phys. Rev. C 2, 1058 (1970).

[ESK71] K. Eskola, P. Eskola, M. Nurmia, and A. Ghiorso, Phys. Rev. C 4, 632 (1971).

[ESK73] P. Eskola, Phys. Rev. C 1, 280 (1973).

[ESK75] P. Eskola, Ph.D. thesis, University of Helsinki, 1975.

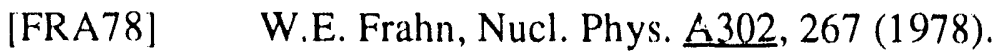

[FRI81] G. Friedlander, J.W. Kennedy, E.S. Macias, and J.M. Miller, Nuclear and Radiochemistry, Third Edition (John Wiley and Sons, New York 1981).

[FRI83] W.A. Friedman, Phys. Rev. C 27, 569 (1983). 
[GÄG86] H. Gäggeler, W. Brüchle, M. Brügger, M. Schädel, K. Sümmerer, G. Wirth, J.V. Kratz, M. Lerch, Th. Blaich, G. Herrmann, N. Hildebrand, N. Trautmann, D. Lee, K.J. Moody, K.E. Gregorich, R.B. Welch, G.T. Seaborg, D.C. Hoffman, W.R. Daniels, M.M. Fowler, and H.R, von Gunten, Phys. Rev. C 33, 1983 (1986).

[GAL69] J. Galin, B. Gatty, M. Lefort, J. Peter, X. Tarrago, and R. Basile, Phys. Rev. 182, 1267 (1969).

[GAL70] J. Galin, D. Guerreau, M. Lefort, J. Peter, X. Tarrago, and R. Basile, Nucl. Phys. A159, 461 (1970).

[GAL76] J. Galin, J. Phys. (Paris) Colloque C 5, 83 (1976).

[GEN89] General Electric Company, Nuclides and Isotopes, Fourteenth Edition (General Electric, San Jose 1989).

[GHI67] A. Ghiorso, T. Sikkeland, and M.J. Nurmia, Phys. Rev, Lett. 18, 401 (1967).

[GLE83] N.K. Glendenning, Direct Nuclear Reactions (Academic Press, New York 19ð3).

[GRE89] K.E. Gregorich and G.T. Seaborg, Lawrence Berkeley Laboratory Preprint LBL-27947

[GRE85] K.E. Gregorich, Ph.D. thesis, University of California, 1985.

[GRO81] D.H.E. Gross and K.M. Hartmann, Phys. Rev. C 24, 2526 (1981).

[HAH74] R.L. Hahn, P.F. Dittner, K.S. Toth, and O.L. Keller, Phys. Rev. C 10 , 1889 (1974).

[HAL86] H.L. Hall (unpublished).

[HAR83] B.G. Harvey and M.J. Murphy, Phys. Lett. 130B, 373 (1983).

[HER79] G. Herrmann, Nature 280, 543 (1979).

[HIG60] G.H. Higgens, The Radiochemistry of the Transcurium Elements, NAS-NS-3031 (1960). 
[HOF80] D.C. Hoffman, D. Lee, A. Ghiorso, M. Nurmia, and K. Aleklett, Phys. Rev. C 22, 1581 (1980).

[HOF85] D.C. Hoffman, Americium and Curium Chemistry and Technology (D. Reidel Publishing Company, Dordecht, 1985), p. 241-250.

[HOF85A] D.C. Hoffman, M.M. Fowler, W.R. Daniels, H.R. von Gunten, D. Lee, K.J. Moody, K. Gregorich, R. Welch, G.T. Seaborg, W. Brüchle, M. Brügger, H. Gäggeler, M. Schädel, K. Sümmerer, G. Wirth, Th. Blaich, G. Herrmann, N. Hildebrand, J.V. Kratz, M. Lerch, and N. Trautmann, Phys. Rev. C 31, 1763 (1985).

[HOF86] D.C. Hoffman, Lawrence Berkeley Laboratory Preprint LBL-21384 (1986).

[HOF88] D.C. Hoffman, in Proceedings of the Second International Conference on Nuclear and Radiochemisiry, Brighton, 1988, also Lawrence Berkeley Laboratory Preprint LBL-26277 (1987).

[HOF88A] D.C. Hoffman, "Transfer Reactions with Transuranium Elements," 3rd Chemical Congress of North America, Award Symposium for Günter Herrmann, June 6-10, 1988, Toronto, Canada, also Lawrence Berkeley Laboratory Preprint LBL-29502 (1990).

[HOF90] D.C. Hoffman, D.M. Lee, K.E. Gregorich, M.J. Nurmia, R.B. Chadwick, K.B. Chen, K.R. Czerwinski, C.M. Gannett, H.L. Hall, R.A. Henderson, B. Kadkhodayan, S.A. Kreek, and J.D. Leyba, Phys. Rev. C 41, 631 (1990).

[HOR69] E.P. Horwitz, C.A. Bloomquist, and D.J. Henderson, J. Inorg. Nucl. Chem. 31,1149 (1969).

[HOR69A] E.P. Horwitz, C.A. Bloomquist, D.J. Henderson, and D.E. Nelson, J. Inorg. Nucl. Chem. 31, 3255 (1969).

[HOR72] E.P. Horwitz and C.A. Bloomquist, J. Inorg. Nucl. Chem. 34, 3851 
(1972).

[HUB80] F. Hubert, A. Fleury, R. Bimbot, and D. Gardes, Ann. Phys. 5, 1 (1980).

[JAC56] J.D. Jackson, Can. J. Phys. 31, 767 (1956).

[KAD90] B. Kadkhodayan, unpublished (1990).

[KAU59] R. Kaufmann and R. Wolfgang, Phys. Rev. Lett. 3, 232 (1959).

[KAU60] R. Kaufmann and R. Wolfgang, Phys. Rev. 121, 192 (1960).

[KRA77] J.V. Kratz, H. Ahrens, W. Bögl, W. Brüchle, G. Franz, M. Schädel,

I. Warnecke, G. Wirth, G. Klein, and M. Weis, Phys. Rev. Lett. 39, 984 (1977).

[LEE82] D. Lee, H.R. von Gunten, B. Jacak, M. Nurmia, Y. Liu, C. Luo, G.T. Seaborg, H.R. von Gunten, and D.C. Yoffman, Phys. Rev. C 25, 286 (1982).

[LEE83] D. Lee, K.J. Moody, M.J. Nurmia, G.T. Seaborg, H.R. von Gunten, and D.C. Hoffman, Phys. Rev. C 27, 2656 (1983).

[LER87] R.G. Leres, Lawrence Berkeley Laboratory Preprint LBL-24808, (1987).

[LEY90] J.D. Leyba, R.A. Henderson, H.L. Hall, C.M. Gannett, R.B. Chadwick K.R. Czerwinski, S.A. Kreek, G.R. Haynes, K.E. Gregorich, D.M. Lee, M.J. Nurmia, and D.C. Hoffman, Phys. Rev. C 41, 2092, (1990).

[LEY90A] J.D. Leyba, R.A. Henderson, H.L. Hall, K.R. Czerwinski, B.A. Kadkhodayan, S.A. Kreek, E.K. Brady, K.E. Gregorich, D.M. Lee, M.J. Nurmia, and D.C. Hoffman, submitted to Phys. Rev. C.

[LEY90B] J.D. Leyba (unpublished).

[MAS71] D.L. Massart, Cation-Exchange Techniques in Radiochemistry, NAS-NS-3113 (1971).

[MCF82] R.M. McFarland, Ph.D. thesis, University of California, 1982.

[MCF83] R.M. McFarland, A. Ghiorso, and G.T. Seaborg, Lawrence Berkeley Laboratory Preprint LBL-15529 1983. 
[MOR81] L.G. Moretto, Rep. Prog. Phys. 44, 533 (1981).

[MOO83] K.J. Moody, Ph.D. thesis, University of California, 1983.

[MOO86] K. Moody, D. Lee, R.B. Welch, K.E. Gregorich, G.T. Seaborg, R.W. Lougheed, and E.K. Hulet, Phys. Rev. C 33, 1315 (1986).

[MYE74] W.D. Myers and W.J. Swiatecki, Ann. Phys. 84, 186 (1974).

[MYE80] W.D. Myers, Droplet Model of Atomic Nuclei, IFI/Plenum, New York (1980).

[MYL11] F. Mylius and C. Hüttner, Bec. Deut. Chem. Ges. 44, 1315 (1911).

[NOR70] R.C. Northcliffe and R.F. Schilling, Nucl. Data Tables A7, 233 (1970).

[NÖR75] W. Nörenberg, Z. Phys. A274, 241 (1975).

[PAL88] C.E.A. Palmer, H.L. Hall, P.A. Baisden, D.C. Hoffman, and D.M. Lee, LLNL Nuclear Chemistry Division Annual Report FY 1988, Report No. UCAR 10062-88 (1988).

[PEP57] P.F. Peppard, G.W. Mason, J.L. Maier, and W.J. Driscoll, J. Inorg. Nucl. Chem. 4, 334 (1957).

[RAN79] J. Randrup, Nucl. Phys. A327, 490 (1979).

[RAN86] J. Randrup, Nucl. Phys. A452, 105 (1986).

[RIE79] C. Riedel and W. Nörenberg, Z. Phys A290, 385 (1979).

[ROU69] J.T. Routti and S.G. Prussin, Nucl. Inst. Meth. 72, 125 (1969).

[SCH77] W.U. Schröder and K.A. Dill, Ann. Rev. Nucl. Sci. 27, 465 (1977).

[SCH78] M Schädel, J.V. Kratz, H. Ahrens, W. Brüchle, G. Franz, H. Gäggeler, I. Warnecke, G. Wirth, G. Herrmann, N. Trautmann, and W. Weis, Phys. Rev. Lett. 41, 469 (1978).

[SCH81] W.U. Schröder, J.R. Huizenga, and J. Randrup, Phys. Lett. 28B, 355 (1981).

[SCH82] M. Schädel, W. Brüchle, H. Gäggeler, J.V. Kratz, K. Sümmerer, G. Wirth, G. Herrmann, R. Stakemann, G. Tittel, N. Trautmann, 
J.M. Nitschke, E.K. Hulet, R.W. Lougheed, R.L. Hahn, and

R.L. Ferguson, Phys. Rev. Lett. $\underline{48}, 852$ (1982).

[SCO77] D.K. Scott, Lawrence Berkeley Laboratory Preprint LBL-7111 (1977).

[SEE67] P.A. Seeger and R.C. Perisho, Los Alamos Scientific Laboratory Report LA-3751, September 1967.

[SIE71] P.J. Siemens, J.P. Bondorf, D.H.E. Gross, and F. Dickmann, Phys. Lett. 36B, 24 (1971).

[SIK68] T. Sikkeland, A. Ghiorso, and M.J. Nurmia, Phys. Rev. 172, 1232 (1968).

[SIK72] T. Sikkeland, N.H. Shafrir, and N. Trautmann, Phys. Lett $\underline{42 B}, 201$ (1972).

[SMI56] H.L. Smith and D.C. Hoffman, J. Inorg. Nucl. Chem. 3, 243 (1956).

[STE61] P.C. Stevenson and W.E. Nervik, The Radiochemistry of the Rare Earth's. Scandium, Yttrium and Actinium, NAS-NS-3020 (1961).

[STE66] P.C. Stevenson, Processing of Counting Data, NAS-NS-3109 (1966).

[TÜR89] A Türler, Ph.D. thesis, University of Bern, 1989.

[UDA82] T. Udagawa, D. Price, and T. Tamura, Phys. Lett. 118B, 45 (1982).

[VAN84] R. Vandenbosch, A. Lazzarini, D. Leach, P.K. Lock, A. Ray, and A. Seamster, Phys. Rev. Lett. 52, 1964 (1984).

[WAL84] F.W. Walker, D.G. Miller, and F. Feiner, Chart of the Nuclides, Thirteenth Edition, General Electric, San Jose, 1984.

[WEI40] V.F. Weisskopf and D.H. Ewing, Phys. Rev. 57, 472 (1940).

[WEL85] R.B. Welch, Ph.D. thesis, University of California, 1985.

[WEL87] R. Welch, K.J. Moody, K.E. Gregorich, D.M. Lee, and G.T. Seaborg, Phys. Rev. C 35, 204 (1987).

[WIL80] W.W. Wilcke, J.R. Birkelund, H.J. Wollersheim, A.D. Hoover, J.R. Huizenga, W.U. Schröder, and L.E. Tubbs, At. Data Nucl. Data 
Tables, 25, 389 (1980).

[WIL80A] J. Wilczynski, K. Siwek-Wilczynska, J. van Driel, S. Gonggrijp,

D.C.J.M. Hageman, R.V.F. Janssens, J. Lukasiak, and R.H. Siemssen, Phys. Rev. Lett. 45, 606 (1980).

[WIN63] J.W. Winchester, J. Chromatog. 10, 502 (1963). 

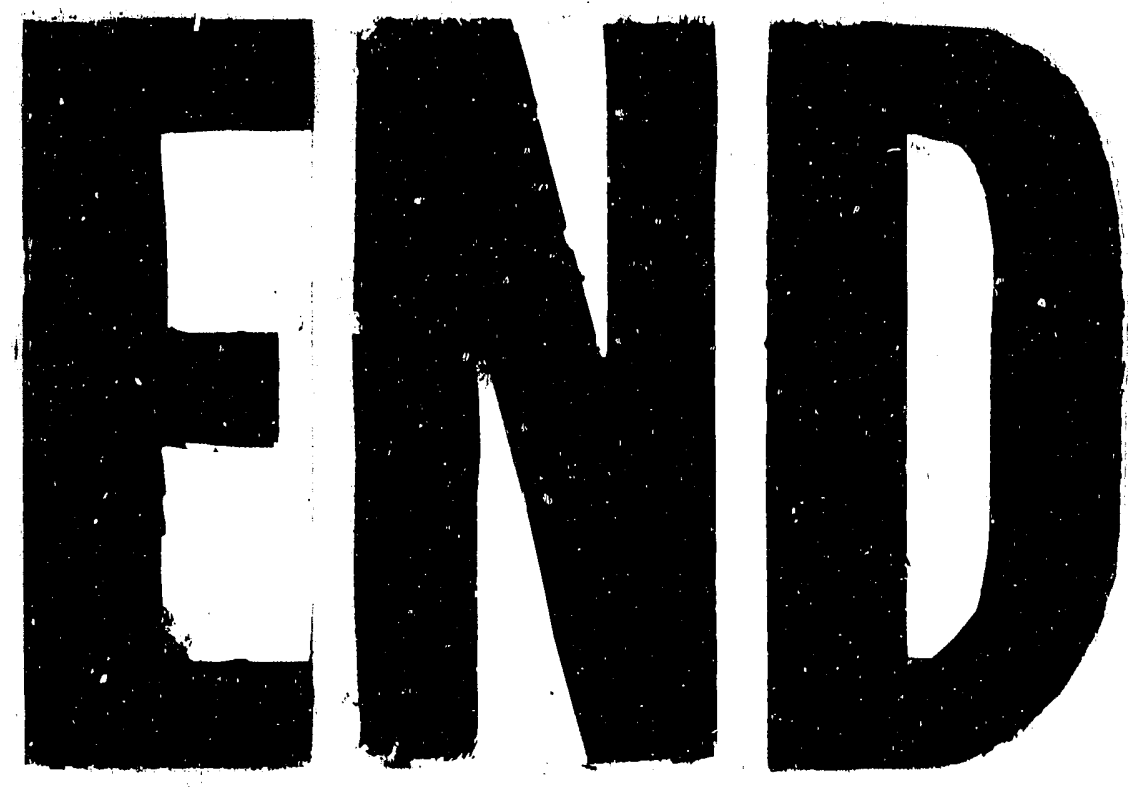

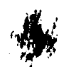
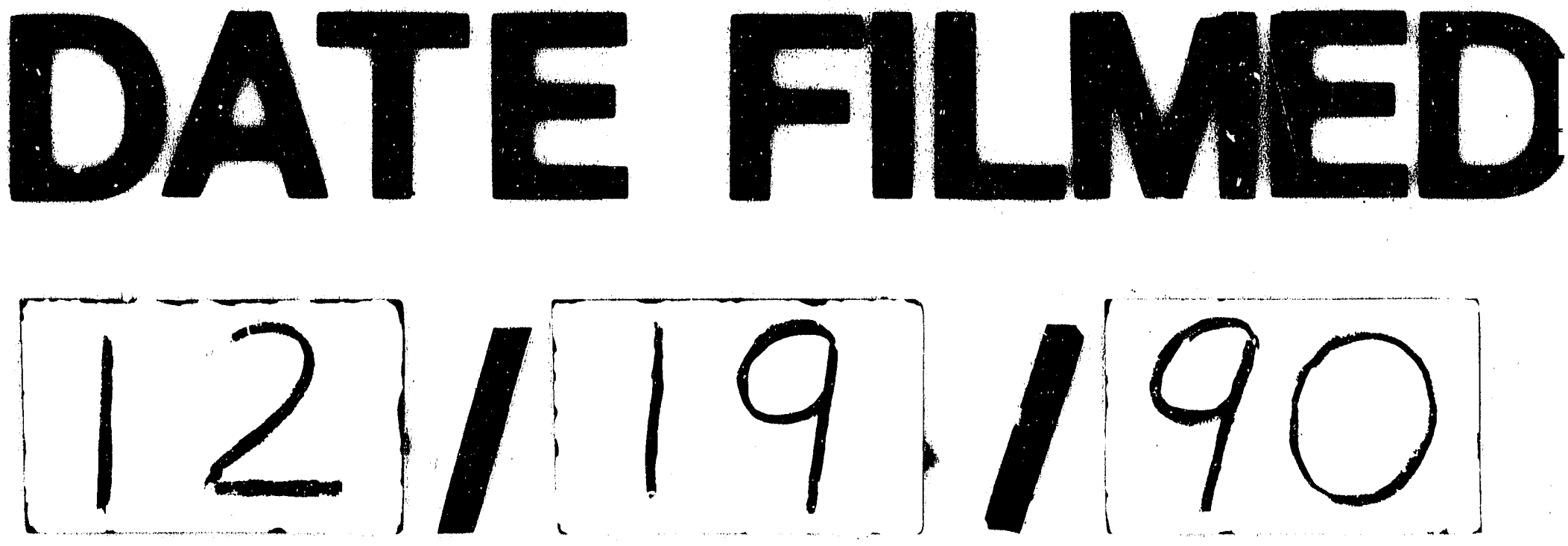
